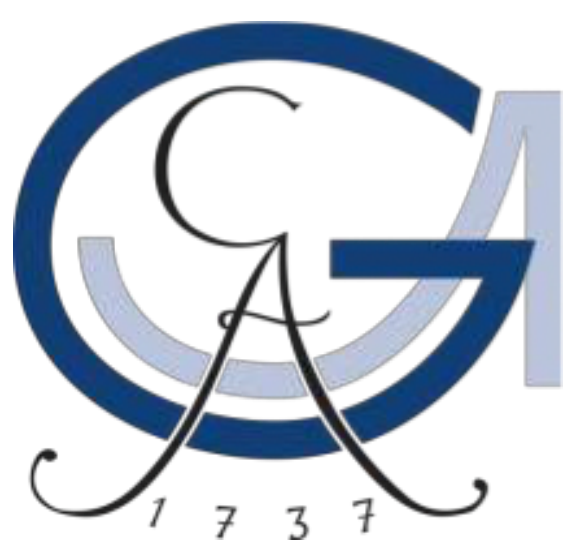

\title{
Transcriptome maps of general eukaryotic RNA degradation factors and identification and functional characterization of the novel mRNA modification $\mathrm{N}^{3}$-methylcytidine
}

\author{
Dissertation \\ for the award of the degree \\ "Doctor rerum naturalium" \\ of the Georg-August-Universität Göttingen
}

within the doctoral program
"Molecular Biology of Cells"
of the Georg-August University School of Science (GAUSS)

submitted by

Katharina Bettina Hofmann

from Würzburg

Göttingen 2019 

Thesis Committee

Prof. Dr. Patrick Cramer

Prof. Dr. Reinhard Lührmann

Prof. Dr. Claudia Höbartner
Max-Planck-Institute for Biophysical Chemistry, Göttingen

Max-Planck-Institute for Biophysical Chemistry, Göttingen

Julius-Maximilans-University, Würzburg

\section{Members of the Examination Board}

Referee:

Prof. Dr. Patrick Cramer, Max-Planck-Institute for Biophysical Chemistry, Göttingen

$2^{\text {nd }}$ Referee:

Prof. Dr. Reinhard Lührmann, Max-Planck-Institute for Biophysical Chemistry, Göttingen

\section{Further members of the Examination Board}

Prof. Dr. Markus Bohnsack

Prof. Dr. Lutz Walter

Prof. Dr. Henning Urlaub
Department of Molecular Biology, University Medical Center, Göttingen

German Primate Center, Göttingen

Max-Planck-Institute for Biophysical Chemistry, Göttingen 



\section{Promovierenden-Erklärung \\ Georg-August-Universität Göttingen}

Name: Hofmann, Katharina Bettina

Anschrift: Otto-Lauffer-Str. 34, 37077 Göttingen

Ich beabsichtige, eine Dissertation zum Thema "Transcriptome maps of general eukaryotic RNA degradation factors and identification and functional characterization of the novel mRNA modification $N^{3}$-methylcytidine" an der Georg-August-Universität Göttingen und dem Max-Planck-Institut für Biophysikalische Chemie anzufertigen. Dabei werde ich von Herrn Prof. Dr. Patrick Cramer betreut.

Ich gebe folgende Erklärung ab:

1. Die Gelegenheit zum vorliegenden Promotionsvorhaben ist mir nicht kommerziell vermittelt worden. Insbesondere habe ich keine Organisation eingeschaltet, die gegen Entgelt Betreuerinnen und Betreuer für die Anfertigung von Dissertationen sucht oder die mir obliegenden Pflichten hinsichtlich der Prüfungsleistungen für mich ganz oder teilweise erledigt.

2. Hilfe Dritter wurde bis jetzt und wird auch künftig nur in wissenschaftlich vertretbarem und prüfungsrechtlich zulässigem Ausmaß in Anspruch genommen. Insbesondere werden alle Teile der Dissertation selbst angefertigt; unzulässige fremde Hilfe habe ich dazu weder unentgeltlich noch entgeltlich entgegengenommen und werde dies auch zukünftig so halten.

3. Die Richtlinien zur Sicherung der guten wissenschaftlichen Praxis an der Universität Göttingen werden von mir beachtet.

4. Eine entsprechende Promotion wurde an keiner anderen Hochschule im Inoder Ausland beantragt; die eingereichte Dissertation oder Teile von ihr wurden nicht für ein anderes Promotionsvorhaben verwendet.

Mir ist bekannt, dass unrichtige Angaben die Zulassung zur Promotion ausschließen bzw. später zum Verfahrensabbruch oder zur Rücknahme des erlangten Grades führen.

Göttingen, den 31.03.2019

Katharina B. Hofmann 


\section{Acknowledgements}

First, I would like to thank my supervisor Prof. Dr. Patrick Cramer for the opportunity to work on such interesting and state-of-the-art projects. Furthermore, I would like to express my gratitude for his supervision, guidance and all the discussions throughout my PhD time.

Moreover, I am very thankful for the support and discussions to the members of my thesis committee, Prof. Dr. Reinhard Lührmann and Prof. Dr. Claudia Höbartner. I would also like to thank Prof. Dr. Markus Bohnsack, Prof. Dr. Lutz Walter and Prof. Dr. Henning Urlaub for being part of my examination board and their time to evaluate this dissertation.

A special thanks goes to Salma Sohrabi-Jahromi from the group of Dr. Johannes Söding. She performed all the bioinformatic analysis on the degradation project and thereby provided the basis for data interpretation leading to many results for this dissertation. I am very happy to see that all our efforts finally turned into a nice manuscript.

I would like to thank the GGNB Office for their support and for providing a fantastic basis for graduation. I would also like to thank the members of the GGNB Program "Molecular Biology of Cells" for the great retreats, good scientific discussions and leisure activities.

I would like to thank all current and former members of the Cramer group for scientific discussions and a nice working atmosphere. I am particularly grateful to Svetlana for sharing the lab with me and the great time together. A special thanks goes to Janine, Kirsten, Kerstin, Petra, Thomas, Sigurd, Andrea, Angelika, and Manuela for lab infrastructure, organization and technical support. 
I am very happy that I can count many of my current and former colleagues to my friends, especially Carina, Ania, Livia, Kristina, Svetlana, Kerstin, Jinmi, and Saskia. I want to thank them for a great time in Göttingen and especially for our fantastic trips to Krakow, Bremen, Barcelona, Berlin, Hamburg, Dublin, Goslar, Lübeck, Brocken, and of course New Zealand. I am very thankful to all my friends, who supported me during my PhD and visited me in Göttingen, particularly Susi, Ina, Carina, Lisa, Sandra, Janine, and Dolores.

I would like to thank Kerstin, Ania, Svetlana, and Jannik for critical reading of this dissertation and their valuable feedback.

I would like to express my deepest gratitude to my family for their constant support and understanding. Last but not least, I am most grateful to Jannik, who cheered my up in difficult times and for his support, patience, understanding and love. 


\section{Publications associated with this dissertation}

Sohrabi-Jahromi S*, Hofmann KB* ${ }^{*}$, Boltendahl A, Roth C, Gressel S, Baejen C, Soeding J, Cramer P. 2019. Transcriptome maps of general eukaryotic RNA degradation factors. Elife 8. doi: 10.7554/eLife.47040

*These authors contributed equally to this work.

Author contributions: SSJ designed and performed bioinformatics analysis. $\mathrm{KH}$ optimized and carried out PAR-CLIP experiments. AB, CB, SG carried out PAR-CLIP experiments. CR contributed to bioinformatics analysis. JS and PC designed and supervised research. SSJ, KH, JS and PC prepared the manuscript, with input from other authors.

Hofmann KB, Villamil GJ, Schwalb B, Sohrabi-Jahromi S, Söding J, Cramer $P$. Mapping and functional characterization of the $N^{3}$-methylcytidine mRNA modification.

Current author list.

Current author contributions: $\mathrm{KH}$ generated all experimental data. GV did bioinformatic analysis on 4tU-seq data under supervision of BS. SSJ did colocalization analysis under supervision of JS. KH and PC wrote the manuscript with input from all authors.

(Manuscript in preparation) 


\section{Other publications derived during the time of this dissertation}

Jones JL, Hofmann KB, Cowan AT, Temiakov D, Cramer P, Anikin M. 2019. Yeast mitochondrial protein Pet111p binds directly to two distinct targets in COX2 mRNA, suggesting a mechanism of translational activation. J Biol Chem 294(18):1-20. doi:10.1074/jbc.RA118.005355

Author contributions: JJ: validation, investigation, visualization, methodology. $\mathrm{KH}$ : data curation, formal analysis, investigation, visualization, methodology, writing-original draft. AC: data curation, investigation. DT: investigation; methodology, writing-original draft, writing-review and editing. PC: conceptualization, supervision, funding acquisition, validation, methodology, writing-original draft. MA: conceptualization, data curation, supervision, funding acquisition, validation, investigation, methodology, writing-original draft, writingreview and editing

Vos SM*, Pöllmann D*, Caizzi L, Hofmann KB, Rombaut P, Zimniak T, Herzog F, Cramer P. 2016. Architecture and RNA binding of the human negative elongation factor. Elife 5:1-27. doi:10.7554/eLife.14981

*These authors contributed equally to this work.

Author contributions: SMV: conception and design, acquisition of data, analysis and interpretation of data, drafting or revising the article. DP: acquisition of data, analysis and interpretation of data, drafting or revising the article. LC: acquisition of data, analysis and interpretation of data, drafting or revising the article. $\mathrm{KH}$ : acquisition of data, analysis and interpretation of data, drafting or revising the article. PR: acquisition of data, analysis and interpretation of data. TZ: acquisition of data, analysis and interpretation of data. $\mathrm{FH}$ : acquisition of data, analysis and interpretation of data. PC: conception and design, analysis and interpretation of data, drafting or revising the article 
The following chapters and sections were taken from Sohrabi et al.:

3.3 Data analysis

3.3.1 PAR-CLIP data pre-processing

3.3.2 Transcript class enrichment

3.3.3 Metagene analysis

3.3.4 Co-occupancy

3.3.5 Co-localization

3.3.6 Codon-enrichment analysis

3.3.7 Relating occupancies to various transcript features

3.3.8 Motif enrichment analysis

4.1 Results

4.1.1 Degradation factors exhibit transcript class specificity

4.1.1.1 RNA end- processing complexes differ in their targets

4.1.1.2 The exosome and surveillance factors

4.1.2 Distinct factor distribution along mRNA

4.1.3 Surveillance of aberrant nuclear non-coding RNA

4.1.4 Interactions between RNA processing machineries

4.1.5 $5^{\prime}$ degradation machinery senses translation efficiency

4.1.6 Decapping factors are enriched upon RNA degradation

The following elements have been adapted to the format of this dissertation and may deviate from Sohrabi et al.:

- Numbering of figures and tables

- References to figures and tables 
The following figures, figure legends, and table were taken from Sohrabi et al. (manuscript table and figure number shown in parenthesis):

Figure 7: Number of PAR-CLIP cross-link sites and replicate correlation. (adapted from Figure 1 and Figure 1 - figure supplement 1 )

Figure 8: Distribution of degradation factor cross-link sites over the yeast transcriptome. (Figure 2)

Figure 9: Metagene analysis of degradation factor binding on protein-coding mRNAs. (Figure 3)

Figure 10: Surveillance of aberrant nuclear non-coding RNA by components of the exosome and the TRAMP4 complex. (Figure 4)

Figure 11: Global co-occupancy and co-localization analysis reveals unexpected cooperation between factors from different complexes and pathways. (Figure 5)

Figure 12: Binding preferences reveal a link between decapping-mediated degradation and translation. (Figure 6)

Figure 13: Location and recruitment of the decapping complex Dcp1/Dcp2 and decapping enhancers Edc3, Dhh1, and Edc2. (Figure 7)

Figure 28: Different transcript classes have comparable U-content. (Figure 1 figure supplement 2)

Figure 29: Metagene profiles of yeast RNA degradation factors centered on translation start and stop sites in comparison to TIF-annotated TSS and pA sites. (Figure 3 - figure supplement 1 )

Figure 30: Distributions of transcript length, half-life, expression level and transcript optimality for yeast mRNAs. (Figure 6 - figure supplement 1 )

Figure 31: Occupancies of deadenylation factors (Ccr4, Pop2, Not1, Caf40, Pan2, and Pan3) compared to transcript length, optimality, expression level, and half-life. (Figure 6 - figure supplement 2 )

Figure 32: Occupancies of decapping factors (Dcp2, Dcp1, Edc2, Edc3, and Dhh1) compared to transcript length, optimality, expression level, and half-life. (Figure 6 - figure supplement 3 ) 
Figure 33: Occupancy of Xrn1 compared to transcript length, optimality, expression level, and half-life. (Figure 6 - figure supplement 4 )

Figure 34: Occupancies of exosome components (Rrp6, Csl4, Rrp40, Rrp4, and Rrp44) compared to transcript length, optimality, expression level, and half-life. (Figure 6 - figure supplement 5 )

Figure 35: Occupancies for components of the TRAMP complex (Air1, Trf5, Mtr4, Air2, and Trf4) compared to transcript length, optimality, expression level, and half-life. (Figure 6 - figure supplement 6 )

Figure 36: Occupancies for components of the Ski complex (Ski2, Ski3, Ski7, and Ski8) compared to transcript length, optimality, expression level, and half-life. (Figure 6 - figure supplement 7 )

Figure 37: Occupancies for components of the NMD pathway (Upf1, Upf2, Upf3, and Nmd4) compared to transcript length, optimality, expression level, and half-life. (Figure 6 - figure supplement 8 )

Figure 38: Correlation between binding to degradation factors and transcript length, codon-optimality, expression, and half-life. (Figure 6 - figure supplement 9 )

Figure 39: Metagene profiles for subunits of the TRAMP complex on snoRNA genes. (Figure 2 - figure supplement 2)

Figure 40: Comparison of binding profiles on genes containing annotated upstream sense NUTs with all mRNAs. (Figure 3 - figure supplement 2)

Table 13: Overview of RNA processing factors and their respective published PAR-CLIP experiments. (Table supplement 1) 


\section{Table of Contents}

Promovierenden-Erklärung.......................................................................

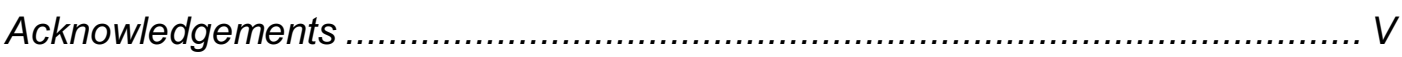

Publications associated with this dissertation .............................................. VII

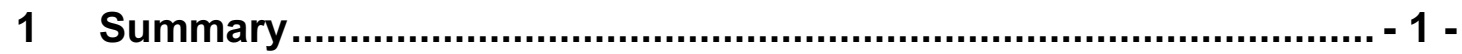

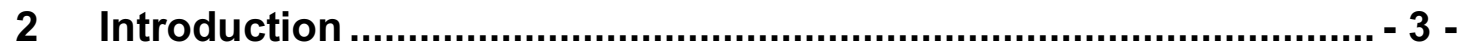

2.1 The central dogma of molecular biology ......................................... - 3 -

2.1.1 Transcription ................................................................... 3 -

2.1.2 Translation ................................................................... 5 -

2.2 The RNA degradation pathways ...................................................... - 8 -

2.2.1 Nuclear surveillance of aberrant non-coding RNAs....................... - 8 -

2.2.2 Cytoplasmic RNA degradation ............................................... - 9 -

2.2.2.1 Deadenylation ....................................................................... 9 -

2.2.2.2 Decapping and exonucleolytic 5' $\rightarrow 3^{\prime}$ RNA degradation ............... - 10 -

2.2.2.3 Exosomal 3' $\rightarrow 5^{\prime}$ RNA degradation ....................................... - 11 -

2.2.2.4 Quality control mechanism for defective mRNA ........................ - 13 -

2.3 The emerging field of epitranscriptomics ...................................... - 14 -

2.3.1 Biogenesis of eukaryotic tRNAs and their function in translation ..... - 14 -

2.3.2 tRNA modifications and their function ....................................... - 14 -

2.3.3 mRNA modifications ............................................................ 16 -

2.3.3.1 $\quad N^{6}$-methyladenosine modification .............................................. -18 -

2.3.3.2 5-methylcytidine modification .............................................. 20 -

2.3.3.3 $\quad N^{1}$-methyladenosine modification ........................................... 20 -

2.3.3.4 Pseudouridine modification ................................................... 21 -

2.3.3.5 $\quad N^{4}$-acetylcytidine modification .............................................. - 22 -

2.3.4 $\quad N^{3}$-methylcytidine modification ................................................ - 23 -

2.3.4.1 $N^{3}$-methylcytidine methyltransferase in S. cerevisiae ................. - 23 -

2.3.4.2 $\quad N^{3}$-methylcytidine methyltransferases in higher eukaryotes.......... - 24 -

2.4 Aims of this thesis ......................................................................... 25 -

2.4.1 Transcriptome maps of general eukaryotic RNA degradation factors- 25 -

2.4.2 Identification and functional characterization of the novel mRNA modification $\mathrm{N}^{3}$-methylcytidine ................................................ - 25 -

3 Materials and Methodology ...................................................... 27 -

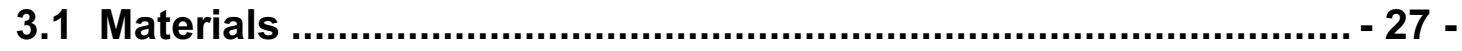

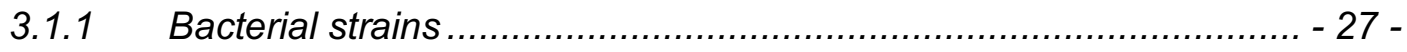


3.1.2 Yeast strains..................................................................... 27 -

3.1.3 Human cell lines ............................................................. - 28 -

3.1.4 Media and supplement.................................................... 29 -

3.1.5 Spike-ins....................................................................... 30 -

3.1.6 Primers and oligonucleotides................................................ 30 -

3.1.7 Thermal cycler programs .......................................................... - 31 -

3.1.8 Plasmids............................................................................ 32 -

3.1.9 Buffers and solutions ...................................................... 32 -

3.1.10 Antibodies................................................................ 33 -

3.2 Experimental methodology ................................................. 34 -

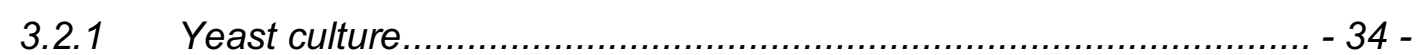

3.2.2 S. cerevisiae strain validation by SDS-PAGE and Western Blot....... - 34 -

3.2.3 Generation of the METTL8-3xFLAG FIp-In TM T-REX ${ }^{\mathrm{TM}} 293$ Cell Line- 34 -

3.2.4 PAR-CLIP....................................................................... 35 -

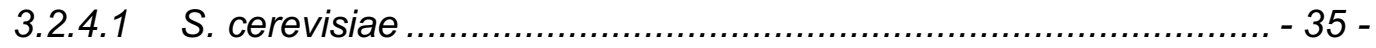

3.2.4.2 Human ...................................................................... -38 -

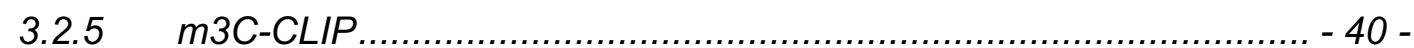

3.2.5.1 S. cerevisiae ................................................................ 40 -

3.2.5.2 Human

3.2.6 4tU-seq in S. cerevisiae ........................................................... 41 -

3.2.7 Protein purification of AlkB and Trm140 ................................. -41 -

3.2.8 RNA demethylation using AlkB ................................................ - 42 -

3.2.9 RNA re-methylation using Trm140 and SAM ............................. - 42 -

3.2.10 Reverse transcription assay and denaturing PAGE ...................... 42 -

3.3 Data analysis ......................................................................... - 43 -

3.3.1 PAR-CLIP data pre-processing........................................... 43 -

3.3.2 Transcript class enrichment ..................................................... - 44 -

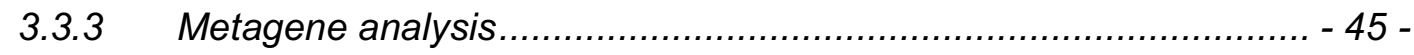

3.3.4 Co-occupancy........................................................................... 46 -

3.3.5 Co-localization ......................................................... 47 -

3.3.6 Codon-enrichment analysis................................................. - 47 -

3.3.7 Relating occupancies to various transcript features ....................... 48 -

3.3.8 Motif enrichment analysis ....................................................... - 49 -

3.3.9 Trm140 PAR-CLIP and m3C-CLIP analysis.............................. - 50 -

3.3.10 Calling of $m 3 C$ modification sites .......................................... 51 -

3.3.11 Calculation of ribosome $P$ site occupancy................................. 51 -

3.3.12 Calculation of translational efficiency on m3C containing codons .... - 51 -

3.3.13 4tU-seq data pre-processing and normalization ........................... 52 - 


\section{Transcriptome maps of general eukaryotic RNA degradation}

factors ..................................................................................... 55 -

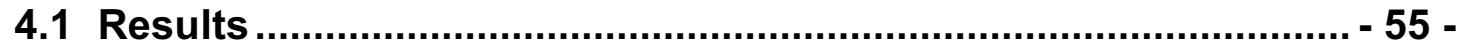

4.1.1 Degradation factors exhibit transcript class specificity................... - 57 -

4.1.1.1 RNA end-processing complexes differ in their targets................ - 57 -

4.1.1.2 The exosome and surveillance factors................................... - 58 -

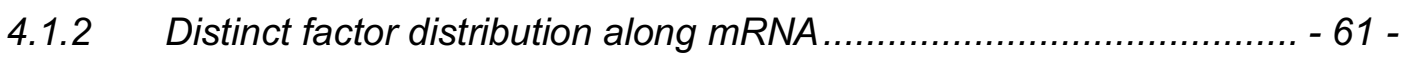

4.1.3 Surveillance of aberrant nuclear non-coding RNA ........................... - 64 -

4.1.4 Interactions between RNA processing machineries ........................ - 66 -

4.1.5 5' degradation machinery senses translation efficiency ................. - 69 -

4.1.6 Decapping factors are enriched upon RNA degradation ................. - 71 -

4.2 Discussion and Outlook .......................................................... - 74 -

4.2.1 Occupancy profiles of general eukaryotic degradation factors give new insights into RNA degradation processes............................... - 74 -

4.2.2 Initial step of RNA decay - mRNA deadenylation ........................... - 74 -

4.2.3 Decapping as first step for RNA degradation from the $5^{\prime}$ end .......... - 76 -

4.2.4 Nuclear RNA surveillance mechanism ..................................... - 77 -

4.2.5 RNA degradation by the exosome complex and auxiliary factors.... - 78 -

4.2.6 Cytoplasmic RNA surveillance by NMD factors............................ - 80 -

4.2.7 Perspectives of mechanistic insights into the RNA degradation process by complementation of the transcriptome maps with functional studies.

4.2.8 Protein-RNA binding studies of eukaryotic degradation factors in response to stress conditions will allow for better functional characterization of the RNA degradation machinery....

4.2.9 Conservation of general RNA degradation factors in human cells.... - 82 -

5 Identification and functional characterization of the novel mRNA modification $N^{3}$-methylcytidine $($ m3C) ........................... - 85 -

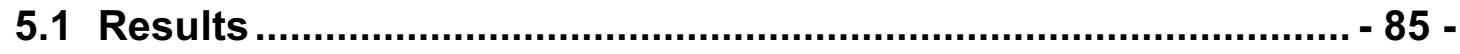

5.1.1 Experimental set-up for Trm140 PAR-CLIP and m3C-CLIP ............ - 85 -

5.1.2 Sequencing coverage of Trm140 PAR-CLIP and m3C-CLIP at established $\mathrm{m} 3 \mathrm{C}$ modification sites........................................... - 87 -

5.1.3 Reverse transcription signature of $m 3 C$ modification ..................... - 89 -

5.1.4 Identification of m3C modification in tRNA Arginine (CCU) .............. - 90 -

5.1.5 Mapping of $m 3 C$ modification sites in $m R N A$............................. - 93 -

5.1.6 Translation efficiency change upon m3C depletion ........................ - 94 -

5.1.7 Co-localization of Trm140 with RNA degradation factors ................ - 97 - 
5.1.8 Depletion of m3C modification alters RNA synthesis rate and half-life. $-98-$

5.1.9 Experimental set-up for PAR-CLIP of METTL8 …...................... - 100 -

5.1.10 Mapping of the m3C modification in human mRNA ..................... - 102 -

5.2 Discussion and Outlook ............................................................ - 104 -

5.2.1 Identification of the m3C modification in tRNA Arginine ................ - 104 -

5.2.2 Effect of m3C loss on other tRNA modifications........................... - 105 -

5.2.3 Functional relevance of the m3C modification............................ - 105 -

5.2.4 Future directions for studies on the identification of $\mathrm{m} 3 \mathrm{C}$ binding proteins in different eukaryotic cells ........................................ - 108 -

5.2.5 Functional implication of the $\mathrm{m} 3 \mathrm{C}$ modification in the context of the epitranscriptome............................................................ - 109 -

5.2.6 Dissecting functional implications of the $\mathrm{m} 3 \mathrm{C}$ modification in $t R N A$ and $m R N A$ in the future

5.2.7 Functional characterization of the $\mathrm{m} 3 \mathrm{C}$ modification in human cells in the future

5.2.8 Technical challenges in the field of epitranscriptomics and future perspectives

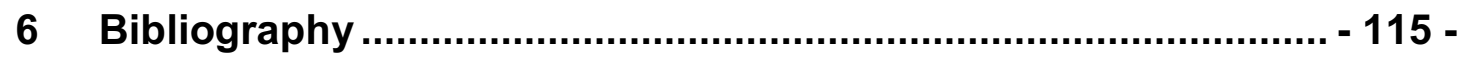

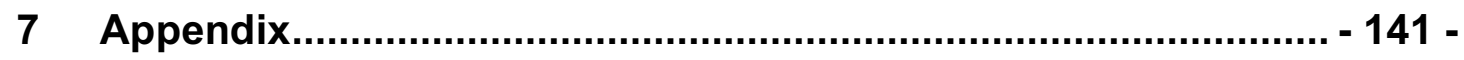

7.1 Supplementary Information.......................................................... - 141 -

7.1.1 Figures ......................................................................... 141 -

7.1.2 Tables.............................................................................. 152 -

7.1.3 Spike-in sequences ........................................................... 155 -

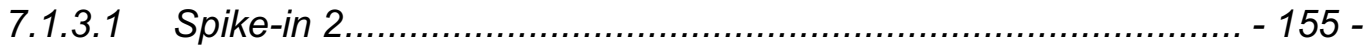

7.1.3.2 Spike-in 4................................................................... 155 -

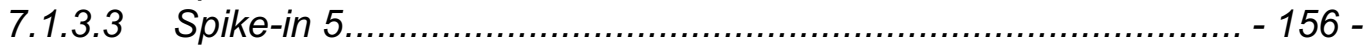

7.1.3.4 Spike-in 8.................................................................. 156 -

7.1.3.5 Spike-in 9................................................................. 157 -

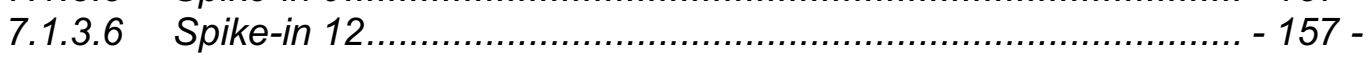

7.2 Abbreviations ........................................................................... 159 -

7.3 List of Figures ................................................................. 165 -

7.4 List of Tables ............................................................................. 167 -

8 Curriculum Vitae .................................................................... - 169 - 


\section{Summary}

The first part of this dissertation focused on the different RNA degradation pathways in the yeast Saccharomyces cerevisiae (S. cerevisiae). We used the method photoactivatable ribonucleoside-enhanced crosslinking and immunoprecipitation (PAR-CLIP) to systematically generate transcriptomewide protein binding profiles for 30 general RNA degradation factors. In-depth bioinformatic analysis and comparison with previously reported PAR-CLIP data provided factor enrichment on different RNA classes and the binding behavior for mRNAs and their associated antisense transcripts. The results also gave insights into how the various degradation complexes, and different subunits in these complexes, may be involved in the processing and degradation of different RNA species. Several conclusions were drawn with respect to degradation pathway selection, new functions for known factors were proposed, and several hypotheses have emerged that may be tested in the future. The generated datasets provide a rich resource for future studies of eukaryotic RNA degradation pathways, mechanisms, and the integration of mRNA metabolism.

The second part of this dissertation addressed the finding of a novel mRNA modification within the emerging field of epitranscriptomics. We performed PAR-CLIP experiments of the $N^{3}$-methylcytidine (m3C) tRNA methyltransferase Trm140 to investigate its mRNA binding in the yeast $S$. cerevisiae. Using this approach in combination with a newly developed method applying anti-m3C antibodies for RNA immunoprecipitation (m3C-CLIP), we were able to call novel $\mathrm{m} 3 \mathrm{C}$ modification sites. Next generation sequencing methods and biochemical verification assays showed the $\mathrm{m} 3 \mathrm{C}$ modification in tRNA Arginine with anticodon CCU in addition to the established modification sites in tRNA Serine and Threonine in yeast. Our genome wide datasets also provided the first evidence that the $\mathrm{m} 3 \mathrm{C}$ modification is present in yeast $\mathrm{mRNAs}$ and we mapped the modification on nucleotide resolution. Functional analyses suggested an effect of m3C mRNA modification on RNA degradation mediated by translation efficiency changes. Mapping of the $\mathrm{m} 3 \mathrm{C}$ modification sites in human RNA revealed a similar localization compared to yeast over the entire 
mRNA with higher occupancy towards the end of protein coding transcripts and within $C G$ rich sequences. 


\section{Introduction}

\subsection{The central dogma of molecular biology}

Genetic information in eukaryotic cells is stored in the nucleus as deoxyribonucleic acid (DNA). The replication and decoding of DNA, one of the most important processes in living cells, is also known as the "central dogma of molecular biology" (Crick, 1970). The conversion of genetic information into proteins is crucial for nearly all cellular functions. This conversion is sub-divided into two steps: transcription and translation. Transcription is the generation of ribonucleic acid (RNA) complementary to a given DNA sequence in the nucleus. During translation, the RNA serves as a template for protein synthesis in the cytoplasm.

\subsubsection{Transcription}

Transcription is performed by large multi-subunit complexes, which are called DNA dependent RNA polymerases (Pol). In eukaryotes, three distinct multi-subunit enzymes are necessary to transcribe all genes into various RNA molecules. Pol I transcribes the $18 \mathrm{~S}, 5.8 \mathrm{~S}$ and $23 \mathrm{~S}$ ribosomal RNA (rRNA). Pol II transcribes many protein-coding messenger RNAs (mRNA) as well as a variety of non-coding RNAs (ncRNA), such as small nuclear RNAs (snRNA), long intergenic non-coding RNAs (lincRNA), and cryptic unstable transcripts (CUT). Pol III transcribes mostly transfer RNAs (tRNA), 5S rRNA and other small RNAs.

The synthesis of mRNAs by Pol II, which are later translated into proteins is completed in a cyclic process. This can be divided into three major steps: initiation, elongation, and termination (reviewed in Hantsche and Cramer, 2016).

The formation of the transcription competent pre-initiation complex (PIC) on the promoter sequence is the first crucial step of Pol II transcription initiation. The PIC contains, in addition to Pol II, the general transcription factors (GTFs) (Sainsbury et al., 2015). Conformational changes of Pol II regulate the 
transition from initiation to elongation (Proudfoot et al., 2002). Rpb1, the largest subunit of Pol II, contains a C-terminal domain (CTD) consisting of multiple tyrosine-serine-proline-threonine-serine-proline-serine (YSPTSPS) heptapeptide repeats (26 in yeast, 52 in mammals) (Corden et al., 1985). These provide a scaffold for co-transcriptional RNA processing factors (Hirose and Manley, 2000; McCracken et al., 1997b). All amino acids of the CTD can be phosphorylated, except proline. The phosphorylation status of the CTD changes dynamically during the transcription cycle ('CTD code') (Buratowski, 2003; Hahn, 2004; Nechaev and Adelman, 2011; Proudfoot et al., 2002), which influences the maturation steps of pre-mRNAs (Fong and Bentley, 2001). A 7methyl-guanosine cap is added to the $5^{\prime}$ end of the nascent RNA when it reaches a length of 20-30 nucleotides (nt) (Rasmussen and Lis, 1993). This process is catalyzed by capping enzymes, which are recruited to the CTD by phosphorylation of the serine 5 (S5) residues (Cho et al., 1997; McCracken et al., 1997a). The $5^{\prime}$ cap is important for RNA stability, nuclear export, and translation initiation (Proudfoot et al., 2002).

Transcription termination is induced by the recruitment of $3^{\prime}$ end processing factors to Pol II via hyper-phosphorylation of the CTD at serine 2 (S2) residues (Ahn et al., 2004; McCracken et al., 1997b). 3' end processing and transcription termination are tightly coupled for eukaryotic protein-coding genes (Whitelaw and Proudfoot, 1986). After the polyadenylation (pA) signal has been transcribed, the nascent transcript gets cleaved directly after the pA site in order to release it from Pol II (Gilmartin and Nevins, 1989). A polyadenylated (polyA) tail is added to the newly generated 3 ' end of the mRNA by the poly-adenosine polymerase (PAP) (Colgan and Manley, 1997; Moore and Sharp, 1985). The polyA tail is required to export the mRNA to the cytoplasm for subsequent translation (Huang and Carmichael, 1996). The released Pol II becomes dephosphorylated and may enter a new transcription cycle. Efficient recycling and reinitiation of Pol II on the same template are facilitated by gene looping and the promoter binding GTFs forming a 'reinitiation platform (Ansari and Hampsey, 2005; Dieci and Sentenac, 1996; Yudkovsky et al., 2000). 
Thus, a full transcription cycle of Pol II produces an mRNA with a $5^{\prime}$ cap and 3' polyA tail, which among other criteria renders the mRNA competent for subsequent translation.

\subsubsection{Translation}

During translation, the mRNA directs the synthesis of a polypeptide chain, which folds into a protein (reviewed in Green and Noller, 1997). This process is catalyzed by a large ribonucleoprotein particle, called the ribosome. The ribosome consists of two subunits, the small $40 \mathrm{~S}$ and large $60 \mathrm{~S}$, together making up the $80 \mathrm{~S}$ ribosome in eukaryotes. Both subunits harbor three binding sites for tRNA as shown in Figure 1: the acceptor ( $\left.A^{\prime}\right)$ site for the incoming aminoacylated tRNA, the peptidyl $(P)$ site for peptide bond formation, and the exit $(E)$ site for holding the deacylated tRNA before leaving the ribosome. The small ribosomal subunit binds the mRNA and the complementary tRNA anticodon stem loop. The open reading frame of mRNA consists of codons, which are made up of three consecutive nucleotides. One codon of the mRNA matches the nucleotide triplet of the tRNA's anticodon loop. Translation is like transcription a cyclic process consisting of initiation, elongation, and termination (reviewed in Green and Noller, 1997).

For translation initiation, the scanning ribosome first has to localize a start codon AUG within the mRNA strand, from which translation can begin. For scanning, the small ribosomal subunit and many auxiliary initiation factors bind to the mRNA in the $5^{\prime}$ untranslated region (5' UTR) between the $5^{\prime}$ cap and the start codon. After start codon recognition, the large ribosomal subunit joins the small ribosomal subunit and the mRNA to form a translation competent $80 \mathrm{~S}$ ribosome (Pestova et al., 2001). 


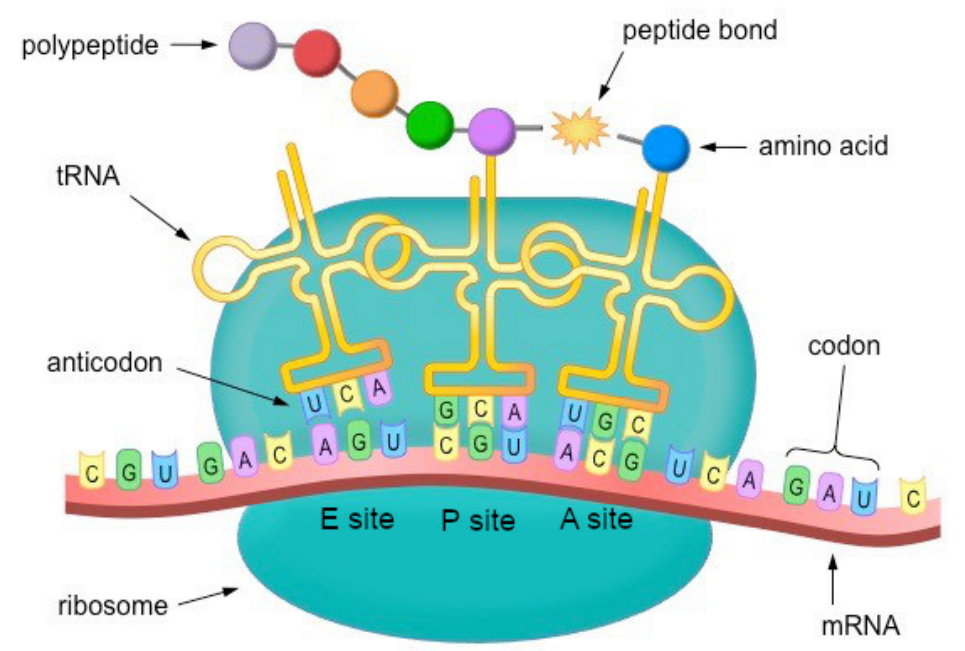

Figure 1: Translation by the ribosome.

The mRNA sequence shown in red serves as a template for protein production via the ribosome shown in green. The codons of the mRNA are complementary to the anticodon of the tRNA loaded with one specific amino acid. During translation the peptide bond formation between the amino acids takes place between the A' site and $P$ site of the ribosome thereby forming the polypeptide sequence. (modified from http://ib.bioninja.com.au/standard-level/topic-2-molecular-biology/27-dna-replicationtranscri/translation.html, 18.02.2019)

For translation elongation, the small ribosomal subunit, together with the mRNA identifies the correct anticodon stem loop of the tRNA (Schmeing et al., $2011,2009)$. Thereby, it contributes to the fidelity of translation via monitoring the decoding process of highly regulated RNA-RNA interactions of codon and anticodon base pairing (Demeshkina et al., 2010). The large ribosomal subunit can bind the acceptor arm of the tRNA and then peptide bond formation between the nascent polypeptide chain in the $P$ site and the aminoacylated tRNA in the $A^{\prime}$ site is catalyzed in the peptidyl transferase center. Both ribosomal subunits and auxiliary elongation factors contribute to translocation, which is the precise movement of tRNA and mRNA through the ribosome one codon at a time (Gao et al., 2009).

Translation termination is the process after stop codon (UAA, UGA, and UAG) recognition in the A' site and separation of the polypeptide chain from the ribosome. Release of the protein requires two release factors binding to the $A^{\prime}$ site in complex with GTP and subsequent GTP hydrolysis to trigger hydrolysis of the polypeptidyl-tRNA. This results in the free protein product with the 
ribosome still bound to mRNA, the deacylated tRNA in the $P$ site, and the release factors in the $A^{\prime}$ site. For further rounds of translation, the ribosome needs to be recycled by disassembling of termination factors and mRNA release (reviewed in Hellen, 2018; Jackson et al., 2012).

In order to get a genome-wide picture of translating ribosomes and an estimation of translation rates and translation efficiency, a method called ribosome-profiling has been developed (Ingolia et al., 2009). By sequencing ribosome protected mRNA fragments, this method allowed monitoring of the ribosome position on the mRNA with single-codon resolution. Comparing translation efficiency with mRNA levels showed its substantial contribution to the dynamic nature of gene expression, which was invisible to measurements of mRNA levels alone. Thus, regulation of transcription and translation contributes to variable production of mRNAs and polypeptide chains. 


\subsection{The RNA degradation pathways}

An active RNA degradation mechanism is implied by comparing RNA production with RNA steady-state levels, which showed more RNA transcription than RNA accumulation in the cell (reviewed in Houseley and Tollervey, 2009). In general, in order to maintain RNA homeostasis each RNA molecule is degraded after a certain lifespan, which can be long for rRNAs or very short for introns or regulatory elements and is precisely controlled for most mRNA transcripts (Miller et al., 2011; Turowski and Tollervey, 2015). Defective RNA molecules in regard to processing, folding, or assembling with proteins are identified and rapidly degraded by the surveillance machinery in order to prevent aberrant protein production (reviewed in Houseley and Tollervey, 2009). Since RNA decay is omnipresent in all cells, it implies a highly accurate controlling mechanism to recognize target RNAs. In addition, upon environmental stimuli such as temperature change or nutrient deprivation, the cell adjusts gene expression by regulating RNA abundance either by changing transcription output or degradation rate (Gasch et al., 2000; Gasch and WernerWashburne, 2002; Jelinsky and Samson, 1999). In principal, RNA degradation can occur from both ends of the transcripts (Parker, 2012), however, the characteristics of a transcript that determine the selection of one or the other decay pathway is a highly studied question in the field.

\subsubsection{Nuclear surveillance of aberrant non-coding RNAs}

Transcription initiation by Pol II exhibits a poor directionality, which leads to pervasive transcription (Core et al., 2008; Neil et al., 2009; Seila et al., 2008; $\mathrm{Xu}$ et al., 2009). In yeast, the phenomenon of bi-directional transcription has been observed by the formation of two adjacent PICs within nucleosomedepleted regions (NDRs) (Murray et al., 2012; Rhee and Pugh, 2012).

Two mechanisms have been shown to restrict the amount of pervasive transcription in eukaryotes: First, gene looping and preferred formation of PICs in one orientation can influence transcription directionality and thereby restrict initiation of divergent ncRNA transcription (Rhee and Pugh, 2012; Tan-Wong 
et al., 2012). Second, nuclear $3^{\prime} \rightarrow 5^{\prime}$ RNA degradation by the exosome and its catalytically active subunit Rrp6 removes excessive transcription products instantly after targeting of the ncRNAs by the Nrd1-Nab3-Sen1 complex (Neil et al., 2009; Schulz et al., 2013; Steinmetz et al., 2001; Xu et al., 2009). The Nrd1-Nab3-Sen1 complex also interacts with the cap binding complex as well as the TRAMP complex (see section 2.2.2.3) (Vasiljeva and Buratowski, 2006). In addition to degradation from the $3^{\prime}$ end, the exonuclease Xrn1 degrades ncRNAs from the $5^{\prime}$ end (van Dijk et al., 2011).

\subsubsection{Cytoplasmic RNA degradation}

\subsubsection{Deadenylation}

RNA degradation is initiated by shortening of the polyA tail by two major deadenylation machineries: the multi-subunit Ccr4-Not complex (Ccr4, Not1, Pop2, Caf40) and the Pan2-Pan3 complex as shown in Figure 2A (Wolf and Passmore, 2014). Deadenylation is one of the rate-limiting steps for mRNA decay, thus, the enzymatic activities of the two mRNA deadenylase complexes make up an important target for the control of mRNA decay (Wolf and Passmore, 2014). Previous investigations regarding decay mechanisms of specific mRNAs showed that selective recruitment of deadenylating enzymes to the substrate mRNA is involved (Finoux and Seraphin, 2006; Goldstrohm et al., 2006; Semotok et al., 2005). Nevertheless, the exact mechanism leading to mRNA deadenylation has not been fully elucidated. A direct link between translation termination and mRNA decay has been revealed by several studies (reviewed in Huch and Nissan, 2014), in particular deadenylation, which is dependent on the poly-adenylate binding protein 1 (Pab1) and Ccr4 (Webster et al., 2018). It has also been shown that translation elongation and mRNA decay are coupled, which depends on the net effect of different cognate tRNA expression termed "codon optimality" (Presnyak et al., 2015). mRNA degradation is enhanced in the presence of rare codons, also non-optimal codons, which are encoded by tRNAs of relatively low abundance (Caponigro et al., 1993). 
Upon translational termination, a stepwise model was proposed for deadenylation by first reducing the average polyA tail length of $90 \mathrm{nt}$ to $50 \mathrm{nt}$ via the Pan2-Pan3 complex, which is then further shortened by the Ccr4-Not complex (Beilharz and Preiss, 2007; Brown and Sachs, 1998; Tucker et al., 2001). The mRNA with an oligo $A$ tail of $10-12 \mathrm{nt}$ can then become a substrate for decapping (Chowdhury et al., 2007; Tharun and Parker, 2001), or it is subjected to exosome catalyzed decay (Bonneau et al., 2009).

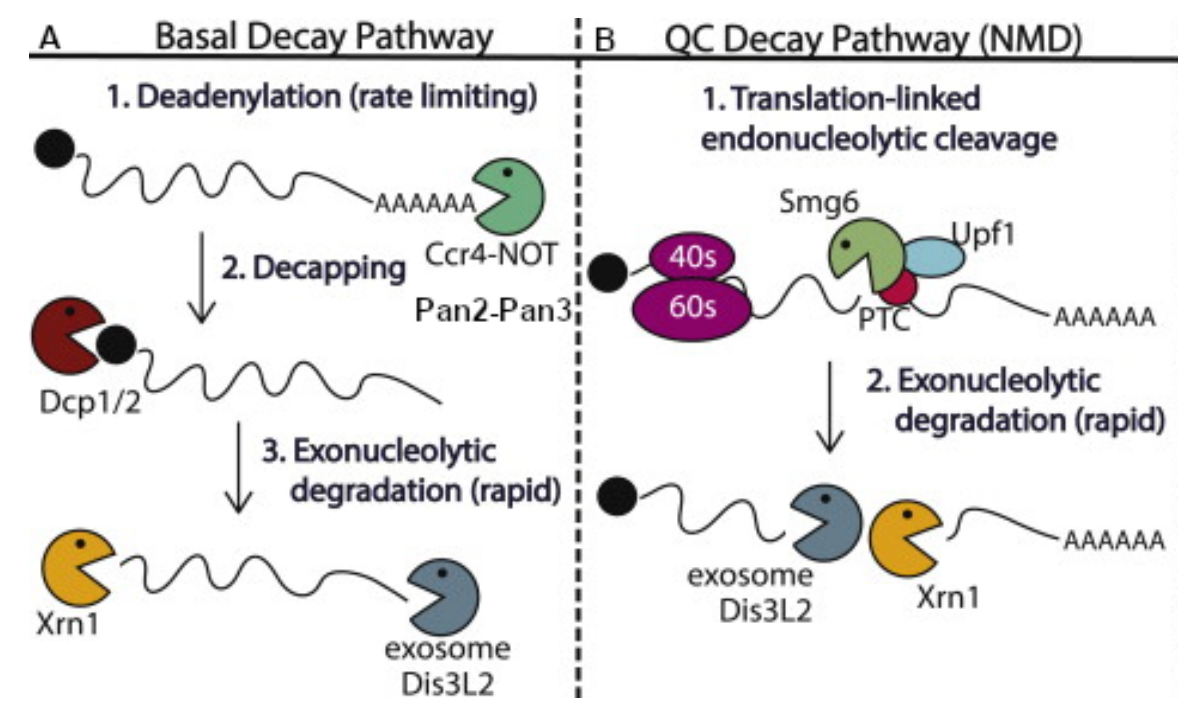

Figure 2: Schematic overview of RNA degradation

A) RNA decay starts with the rate-limiting step of deadenylation via Pan2-Pan3 and Ccr4-Not complexes, followed by decapping via Dcp1 and Dcp2 and exonucleolytic degradation of the transcript by Xrn1 from the $5^{\prime}$ end and the exosome from the $3^{\prime}$ end. B) Quality control decay pathways in particular nonsense mediated decay (NMD) factors such as the Upf1 and Smg6 recognize aberrant mRNAs during translation, mostly in the presence of premature termination codons (PTC), and induce endonucleolytic cleavage, whereupon the fragments are degraded by exonucleases from the 5' end (Xrn1) or 3' end (exosome) (adapted from Abernathy and Glaunsinger, 2015).

\subsubsection{Decapping and exonucleolytic $5^{\prime} \rightarrow 3^{\prime}$ RNA degradation}

The second step in mRNA degradation after deadenylation is removal of the $5^{\prime}$ cap, which protects the RNA from $5^{\prime} \rightarrow 3^{\prime}$ degradation by the exonuclease Xrn1 (Stevens and Poole, 1995). In order to remove the cap, a three-step model is proposed: First, the $5^{\prime}$ cap structure needs to be unprotected by loss of the 
cap-binding complex. Second, recruitment of the decapping enzyme to the mRNA needs to take place, which seems to be regulated by the assembly of a larger decapping complex. Third, catalysis by Dcp2 occurs together with Dcp1, inducing rapid $5^{\prime} \rightarrow 3^{\prime}$ decay of the mRNA by the exonuclease Xrn1 (Hsu et al., 2017; Muhlrad and Parker, 1994). Decapping is highly regulated by decapping enhancers like Dhh1, Edc2 and Edc3. Different mechanisms may trigger decapping by binding RNA and providing a platform for assembly of the decapping machinery, by interfering with translation initiation factors or by stimulating Dcp2 catalytic function. Assembly of the decapping machinery occurs mostly after shortening of the polyA tail, which prevents binding of the protective Pab1, opens the mRNA closed-loop structure, and allows decapping complex formation on the $3^{\prime}$ end of deadenylated mRNA (Caponigro and Parker, 1995; Gallie, 1991; Morrissey et al., 1999). Opening of the mRNA closed-loop structure disrupts the proximity of the $5^{\prime}$ and $3^{\prime}$ ends of the mRNA. This prevents complex formation of translation initiation factors binding to the $5^{\prime}$ cap and Pab1 at the $3^{\prime}$ end, thereby contributing to mRNA expression regulation (Gallie, 1991; Wells et al., 1998).

\subsubsection{Exosomal $3^{\prime} \rightarrow 5^{\prime}$ RNA degradation}

Upon deadenylation, $3^{\prime} \rightarrow 5^{\prime}$ degradation of the mRNA is performed by the exosome and many auxiliary factors (Anderson and Parker, 1998). The exosome is a multi-subunit complex consisting of 10 main subunits. Three small RNA-binding proteins and six members of the RNase $\mathrm{PH}$ protein family form a ring structure similar to bacterial PNPase as shown in Figure 3 (Liu et al., 2006). The Rrp44/Dis3 protein is the catalytically active subunit possessing both an exonuclease and endonuclease domain (Lebreton and Seraphin, 2008; Schaeffer et al., 2009). The exosome complex takes part in many nuclear RNA processing and degradation processes in addition to its functions within the cytoplasm (reviewed in Lykke-Andersen et al., 2009). In order to fulfill its functions in the nucleus, the exosome is additionally bound by the $3^{\prime} \rightarrow 5^{\prime}$ exonuclease Rrp6 as well as RNA binding proteins Rrp47 and Mpp6 (Milligan et al., 2008; Mitchell et al., 2003; Synowsky et al., 2009). 
One of the various co-factors for nuclear exosomal RNA maturation and degradation in eukaryotes is the TRAMP polyadenylation complex (reviewed in Houseley and Tollervey, 2009). This complex consists of two sub-complexes: TRAMP4 (Trf4, Air2 and Mtr4) and TRAMP5 (Trf5, Air1 and Mtr4). The TRAMP complexes harbor a poly-adenosine polymerase (Trf4 or Trf5), a zinc-knuckle putative RNA-binding protein (Air1 or Air2), and an RNA helicase (Mtr4) (Falk et al., 2014). Defective nuclear RNAs can be tagged with a short polyA tail and bound proteins can be detached by the TRAMP complex. This renders the defective RNA a more suitable target for exosomal degradation by the sterically restricted exosome core (Schmidt and Butler, 2013).

The Ski complex is required for cytoplasmic $3^{\prime} \rightarrow 5^{\prime}$ exosomal degradation to feed the RNA into the exosome. The Ski7 protein is stably associated with the cytoplasmic exosome by the Csl4 adapter protein (van Hoof et al., 2002). The Ski2, Ski3, and Ski8 proteins assemble in a discrete protein complex (Brown et al., 2000; Wang et al., 2005) interacting with the Ski7 subunit (Araki et al., 2001; Wang et al., 2005). This interaction is required for $3^{\prime} \rightarrow 5^{\prime}$ degradation of mRNAs by the exosome. The Ski2 subunit is an ATPase, which belongs to the RNA helicase family. It provides energy by ATP hydrolysis to unwind RNA secondary structures and dissociate attached proteins to pass the RNA to the exosome (Johnson and Jackson, 2013).

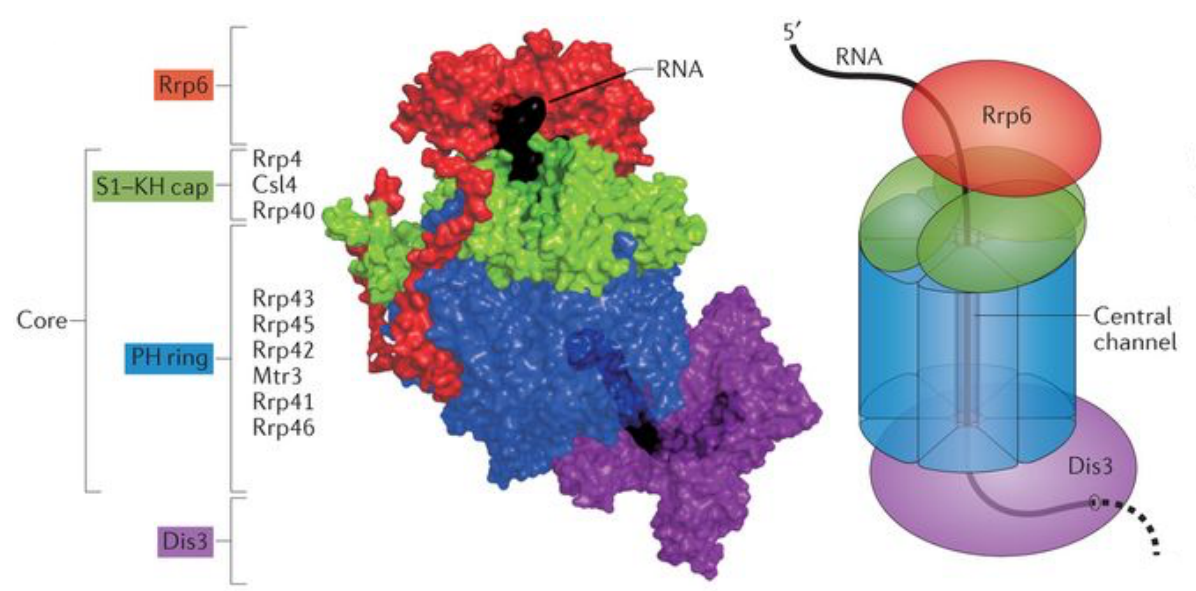

Figure 3: Model of the exosome complex.

Schematic overview of the exosome complex assembly including the nuclear Rrp6 in red and cytoplasmic Dis3 (Rrp44) subunit in violet. The exosome core consists of the cap (Rrp4, Csl4 and Rrp40) depicted in green and the ring domain (Rrp43, Rrp45, Rrp42, Mtr3, Rrp41 and Rrp46) in blue forming a central channel (adapted from Kilchert et al., 2016). 
2.2.2.4 Quality control mechanism for defective mRNA

Aberrant mRNAs need to be determined from the pool of normal mRNAs by adapter proteins. This can be accomplished by interactions of specific adapter proteins with the translation machinery. The aberrant mRNA can then be subjected to one of the RNA decay pathways (Doma and Parker, 2007). mRNAs with aberrant translation termination due to premature translation termination codons are degraded by an mRNA quality control system called nonsense mediated decay (NMD) as depicted in Figure 2B (Losson and Lacroute, 1979). Substrates for NMD are distinguished by the Upf1 protein interacting with the translation termination complex. Subsequently the proteins Upf2 and Upf3 bind to Upf1 thereby enhancing its helicase activity (reviewed in Baker and Parker, 2004; Chakrabarti et al., 2011). Binding of the NMD machinery to an aberrant mRNA has several effects on the fate of the transcript: the polyA tail of the mRNA is subjected to enhanced deadenylation (Cao and Parker, 2003; Mitchell and Tollervey, 2003; Muhlrad and Parker, 1994), rapid deadenylation-independent decapping of the $5^{\prime}$ end (Muhlrad and Parker, 1994), slightly accelerated rates of $3^{\prime} \rightarrow 5^{\prime}$ degradation by the exosome complex after deadenylation (Cao and Parker, 2003; Mitchell and Tollervey, 2003), and inhibition of translation (Muhlrad and Parker, 1999). In addition to its role in degrading aberrant mRNAs, the NMD machinery is involved in the regulation of many biological processes such as stress responses, cell survival and differentiation by controlling the steady state level of normal mRNAs (Goetz and Wilkinson, 2017; Karam et al., 2015; T. Li et al., 2015; Lou et al., 2016; LykkeAndersen and Jensen, 2015; Nelson et al., 2016). It has been shown that the expression regulation of normal mRNAs is achieved by fine tuning of the NMD activity (Huang and Wilkinson, 2012; Karam et al., 2013). 


\subsection{The emerging field of epitranscriptomics}

\subsubsection{Biogenesis of eukaryotic tRNAs and their function in translation}

In S. cerevisiae, Pol III transcribes a total of 275 tRNA genes (Chan and Lowe, 2009). The pre-tRNA transcripts contain a $5^{\prime}$ leader sequence, a $3^{\prime}$ trailer sequence and can harbor an intronic sequence, which need to be removed during biogenesis. After removal of the $5^{\prime}$ leader sequence by RNase P, RNase $Z$ can remove the $3^{\prime}$ trailer sequence at nucleotide 73 and a nucleotidyltransferase is able to add a CCA sequence to the $3^{\prime}$ end of the tRNA (Aebi et al., 1990). Correctly folded and end-processed tRNAs are exported from the nucleus into the cytoplasm using the export factor Los1 (Arts et al., 1998; Lund and Dahlberg, 1998; Sarkar and Hopper, 1998). The conserved family of Sen proteins removes intronic sequences from 61 tRNAs in a process called tRNA splicing in the cytoplasm (Melton et al., 1980; Yoshihisa et al., 2003). The final step of tRNA maturation is aminoacylation by aminoacyl-synthetases (Grosshans et al., 2000; Lund and Dahlberg, 1998; Steiner-Mosonyi and Mangroo, 2004). The correctly loaded tRNAs can then serve as adapter molecules during translation of the mRNA into the amino acid sequence of the protein catalyzed by the ribosome.

\subsection{2 tRNA modifications and their function}

In ncRNAs like rRNA, tRNA and snRNA, more than 100 chemical modifications have been characterized. tRNAs can be highly modified on numerous nucleotides as shown in Figure 4, which is crucial for their specific function in translation (Phizicky and Hopper, 2010). The correct folding of tRNAs into the clover leaf structure (Figure 4) including the acceptor stem, the $D$-loop, the T $\Psi$ C-loop, the anticodon-loop, and the variable loop is ensured by the different RNA modifications installed in the nucleus and cytoplasm depending on the specific modification (reviewed in Hopper, 2013). The tRNA's secondary structure is folded into an inverted L-shaped tertiary structure containing the CCA-acceptor stem at the top and the anticodon-loop at the bottom (Shi and Moore, 2000). The most variably and extensively modified 
nucleotides are in the anticodon loop (ACL) at the wobble position (34) and in the extended anticodon (37) of tRNAs. These modifications may play important roles in decoding precision, efficiency and reading frame maintenance during translation (Björk et al., 2001; Chen C, Huang B, Eliasson M, Ryde'n P, 2011; Esberg et al., 2006; Gerber et al., 1998; Johansson et al., 2008; Murphy and Ramakrishnan, 2004; Urbonavičius et al., 2001; Waas et al., 2007; Weixlbaumer et al., 2007; Yacoubi et al., 2011). Effects on tRNA structure stability are mainly influenced by modifications in the body of the tRNA leading to more rigid or loose parts (reviewed in Lorenz et al., 2017).Incorrectly modified tRNAs or the loss of tRNA modifying enzymes have been implicated in a number of human diseases such as neurological and metabolic diseases as well as several kinds of cancer (reviewed in Torres et al., 2014). A perturbed protein synthesis in response to incorrect codon recognition is considered to be the molecular reason for those diseases (Torres et al., 2014). 


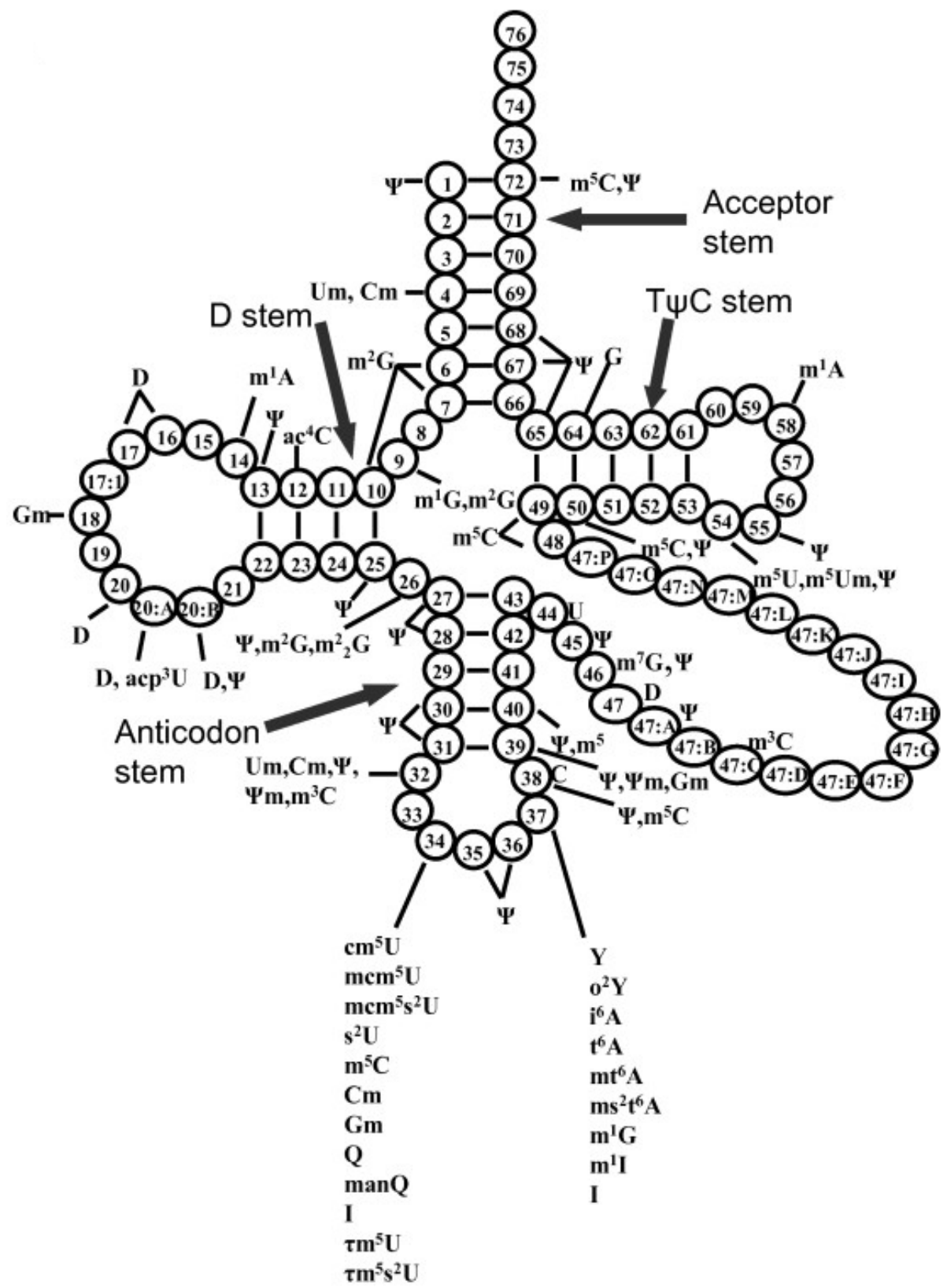

Figure 4: Modified nucleosides in the clover-leaf structure of the eukaryotic tRNA. Each circle represents a nucleotide, numbered from $5^{\prime}-$ to $3^{\prime}-$ end. The clover-leaf structure is divided into the acceptor stem, the D stem, the anticodon stem, the variable stem and the TUC stem loop. Modified nucleosides found at different positions are shown (adapted from Chen et al., 2010).

\subsection{3 mRNA modifications}

In the last ten years a lot of effort has been made to detect RNA modifications in mRNA leading to the emerging field of epitranscriptomics 
(Saletore et al., 2012). The epitranscriptome is defined as a functional change in the mRNA that does not involve changes to the ribonucleotide sequence. The epitranscriptome is analogous to the epigenome, which describes the chemical modifications on DNA and histones. The first modifications described in mRNA were $N^{6}$-methyladenosine (m6A) and 5-methylcytidine (m5C) in the 1970s (Dubin and Taylor, 1975; Schibler et al., 1977). The currently known base modifications in protein-coding transcripts are $\mathrm{m} 6 \mathrm{~A}, \mathrm{~m} 5 \mathrm{C}$, inosine $(\mathrm{I})$, pseudouridine $(\Psi), \quad N^{1}$-methyladenosine $(\mathrm{m} 1 \mathrm{~A})$, 5-hydroxylmethylcytidine (hm5C), and $N^{4}$-acetylcytidine (ac4C) (see Figure 5). Different RNA editing events within the mRNA can change the encoded protein sequence and thereby eventually generate premature stop codons or alter the differential expression levels of micro RNAs (Chawla and Sokol, 2014; Powell et al., 1987). The localization of the different mRNA modifications in eukaryotic proteincoding transcripts is shown in Figure 5. Epitranscriptomic modifications can also influence mRNA stability, structure, splicing, translation and degradation (reviewed in Frye et al., 2016).

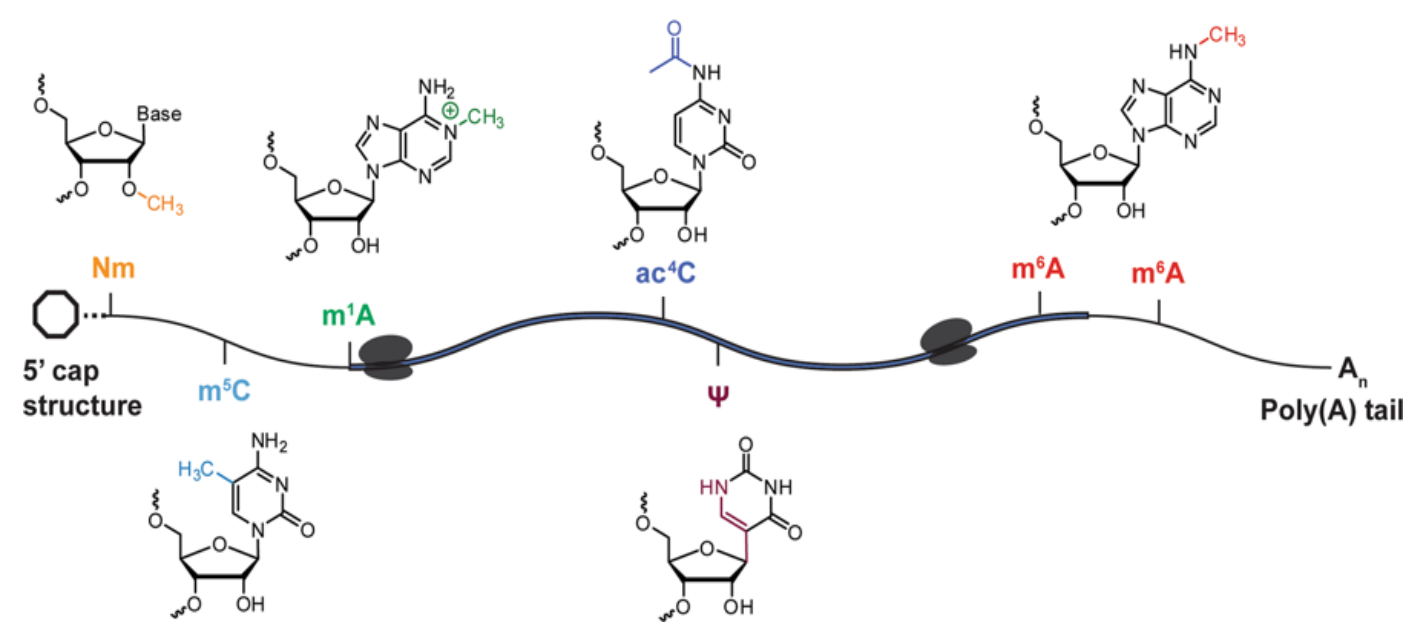

Figure 5: Schematic representation of known chemical modifications mapped in eukaryotic mRNA transcripts.

The protein coding region is highlighted in bold. The to date known base modifications within the mRNA are $N^{6}$-methyladenosine $(\mathrm{m} 6 \mathrm{~A}), 5$-methylcytidine $(\mathrm{m} 5 \mathrm{C})$, inosine $(\mathrm{I})$, pseudouridine $(\Psi), N^{1}$-methyladenosine $(\mathrm{m} 1 \mathrm{~A})$, and $N^{4}$-acetylcytidine (ac4C). Structure of the nucleoside including the chemical modification in color is shown at the position within the transcript, where the modification has been localized. In addition to the base modifications, also the 2'-O-methylation of ribose sugars $(\mathrm{Nm})$ is also shown close to the $5^{\prime}$ cap (adapted from $\mathrm{He}$ and $\mathrm{He}, 2019$ ). 


\subsubsection{1 $\quad N^{6}$-methyladenosine modification}

Development of next-generation sequencing (NGS) techniques enabled a more accurate analysis of the m6A modification and revealed its extent over the transcriptome after its identification in the 1970s (Dubin and Taylor, 1975). The m6A modification is generally found in mRNAs of nearly all eukaryotes from yeast to fruit fly and mouse up to human (Dominissini et al., 2012; Hongay and Orr-weaver, 2011; Schwartz et al., 2013). Antibodies were used to specifically detect the m6A modification in cellular mRNAs (Chen et al., 2015; Dominissini et al., 2012) and by using cross-linking methods coupled to specific mutational signatures, the modification was mapped at single-nucleotide resolution (Linder et al., 2015). These studies revealed over 12,000 possible m6A modification sites in mRNAs and ncRNAs of more than 7,000 human transcripts. However, in yeast the presence of the m6A modifications is reduced to about 1,000 mRNAs and limited to meiosis suggesting a function in translation of certain mRNAs (Schwartz et al., 2013). Mapping of the modification on the mRNA transcript has revealed its localization in humans around stop-codons, long internal exons and in the 3' UTRs (Chen et al., 2015; Dominissini et al., 2012; Saletore et al., 2012). The consensus motif RRACH, which was already proposed in the 1970s, was confirmed by the highly enriched GGACU motif within the sequencing data (Chen et al., 2015; Dominissini et al., 2012; Saletore et al., 2012; Schibler et al., 1977).

The m6A modification is installed by a methylation complex consisting of the methyltransferase METTL3, the putative methyltransferase METTL14, and the regulatory proteins WTAP and KIAA1429 (Liu and Jia, 2014; Ping et al., 2014; Schwartz et al., 2014b). Other m6A methyltransferases seem to be involved in introducing such modifications, as knockdown of the METTL3/METTL14 complex does not completely abolish m6A modification. Furthermore, the known m6A modification sites just overlap partially with the binding sites of METTL3/METTL14 on cellular RNAs indicating the presence of other m6A methyltransferases (Chen et al., 2015; Liu and Jia, 2014; Schwartz et al., 2014b). Indeed, METTL16 was identified to modify pre-mRNAs and various ncRNAs with m6A (Pendleton et al., 2017; Warda et al., 2017). Interestingly, METTL16-dependent m6A modification sites do not have the 
RRACH sequence motif and they occur within introns and at intron-exon boundaries (Pendleton et al., 2017; Warda et al., 2017).

Functional analyses of the $\mathrm{m} 6 \mathrm{~A}$ modification have revealed that the modification may change the secondary structure of the RNA by acting as a molecular switch. This can lead to the display of RNA binding structures or motifs for specific RNA binding proteins (Liu et al., 2015). On a cellular level the $\mathrm{m} 6 \mathrm{~A}$ modification is suggested to be involved in stress response, capindependent translation and regulation of the circadian rhythm of cells (Engel et al., 2018; Fustin et al., 2013; Meyer et al., 2015). In mouse, the m6A modification was observed to keep the omnipotence of the cells thereby regulating embryonic stem cells and was also shown to be involved in the cell cycle regulation of human cells (Dominissini et al., 2012; Y. Wang et al., 2014). Recently, a study revealed a crosstalk between histone modifications and $\mathrm{m} 6 \mathrm{~A}$ modifications to regulate gene expression (Huang et al., 2019). Hereby, METTL14 recognizes the trimethylation of histone $\mathrm{H} 3$ at Lys 36 , which is an established marker for transcription elongation, and facilitates binding of the methyltransferase complex in the vicinity of Pol II to deposit the m6A modification co-transcriptionally (Huang et al., 2019; Kizer et al., 2005).

The m6A modification has been identified to be reversible via the ALKBH5/FTO protein, which erases the mark, thereby allowing for the dynamic nature of the m6A modification associated with different functions in mice and humans (Jia et al., 2011; Zheng et al., 2013). However, not only writer and eraser proteins for $\mathrm{m} 6 \mathrm{~A}$ modification have been identified, but also reader proteins. Those reader proteins specifically bind to the m6A mark thereby influencing the fate of the transcript. The first identified m6A binding proteins share a specific domain, the YT521-B homology (YTH) domain (Zhang et al., 2010). In humans, five YTH domain containing proteins are known, namely YTHDF1, YTHDF2, YTHDF3, YTHDC1, and YTHDC2. These proteins are associated with different functions in cellular processes upon $\mathrm{m} 6 \mathrm{~A}$ recognition in mRNA such as splicing, stability and translation (Kretschmer et al., 2018; Theler et al., 2014; Wang et al., 2015; X. Wang et al., 2014; Xiao et al., 2016). Increased translation of specific m6A containing transcripts has been observed to control anti-tumor immunity in dendritic cells (Han et al., 2019). In Drosophila, 
the $\mathrm{m} 6 \mathrm{~A}$ reader protein $\mathrm{YT521 \textrm {B }}$ has been shown to control neurogenesis and sex determination (Lence et al., 2016).

\subsubsection{5-methylcytidine modification}

In addition to its occurrence in tRNA, rRNA, and the well characterized function in transcription regulation on DNA, the m5C modification was also observed in human and archaeal mRNA (Edelheit et al., 2013; Squires et al., 2012). Bisulfite treatment coupled with NGS enabled mapping of $m 5 C$ and its oxidation products 5 -hydroxymethylcytidine ( $\mathrm{hm} 5 \mathrm{C}$ ) and 5 -formylcytidine ( $\mathrm{f5C}$ ) on a transcriptome-wide level (Booth et al., 2014; Edelheit et al., 2013; Lee and Kim, 2016). In ncRNAs and mRNAs of HeLa cells over 10,000 m5C modification sites were discovered (Squires et al., 2012). Global positional analysis of the $\mathrm{m} 5 \mathrm{C}$ modification revealed an overall distribution in mRNAs with an increased occupancy in $5^{\prime}$ and $3^{\prime}$ UTRs (Squires et al., 2012). This localization of the modifications implies a function in protein translation regulation (Squires et al., 2012). Two additional experiments in fruit flies support this hypothesis (Delatte et al., 2016). The m5C content can also be regulated in human cells by oxidation to $\mathrm{hm} 5 \mathrm{C}$ and $\mathrm{f} 5 \mathrm{C}$, indicating a similar mechanism being present in mammals (Huber et al., 2015).

\subsubsection{3 $\quad N^{1}$-methyladenosine modification}

Specific chemical properties of the m1A modification in mRNA allowed its discovery by using an antibody-based approach to enrich transcripts containing an $\mathrm{m} 1 \mathrm{~A}$ modification. These were later on sequenced to map the modified nucleoside within the transcripts (Dominissini et al., 2016; Li et al., 2016a; Li and Xiong, 2017; Safra et al., 2017b). The number of reported m1A marks in mRNAs varies between each study, ranging from very few (Safra et al., 2017b), to around 800 ( Li et al., 2016a), and up to over 4,000 (Dominissini et al., 2016). m1A is reported to occur at low stoichiometry and in invariable tRNA TYC-loop structures (Safra et al., 2017b). Whereas another study 
reported the average methylation level of an $\mathrm{m} 1 \mathrm{~A}$ containing transcript of approximately $20 \%$ within one mRNA (Dominissini et al., 2016). This study also observed a positional distribution of the m1A marks in the $5^{\prime}$ UTR and the $5^{\circ}$ end of mRNAs (Dominissini et al., 2016). In addition to its presence in the $5^{\prime}$ UTR, others reported occurrence of the m1A modification also in the $3^{\prime}$ UTR (Li et al., 2016a). The last two studies indicated a functional role of the m1A mark in translation regulation as they observed changed methylation patterns in response to different physiological conditions and external stress (Dominissini et al., 2016; Li et al., 2016a). Furthermore, the alpha-ketoglutarate dependent dioxygenase ALKBH3 was identified to erase the m1A modification, thus, making it a reversible modification with a dynamic function (Li et al., 2016a).

\subsubsection{Pseudouridine modification}

Pseudouridine modifications have also been mapped by transcriptomewide sequencing. A chemical labeling approach by using the reactivity of the molecule $\quad N^{3}$-[N-cyclohexyl- $N^{\prime}-\beta$-(4-methylmorpholinium)ethylcarbodiimide (CMC) and pseudouridine introduces a bulky modification (CMC- $\Psi$ ), which causes a reverse transcription stop and therefore allows mapping of the modified nucleotide (Bakin and Ofengand, 1993; Zaringhalam and Papavasiliou, 2016). By using this method, different studies reported the modification to be present in yeast and human mRNAs and ncRNAs (Carlile et al., 2014; X. Li et al., 2015; Lovejoy et al., 2014; Safra et al., 2017a; Schwartz et al., 2014a). Conserved pseudouridine synthases of the Pus family and TRUB1 catalyze isomerization from uridine to pseudouridine with a conversion rate of $0.2-0.6 \%$ identified via mass spectrometry analysis (Carlile et al., 2014; Lovejoy et al., 2014; Safra et al., 2017a; Schwartz et al., 2014a). The high abundance of the pseudouridine mark enables a high regulatory potential, which was observed by altered modification patterns and abundance caused by starvation induced stress response in yeast and human cells (Carlile et al., 2014; X. Li et al., 2015; Schwartz et al., 2014a). Pseudouridylation of nonsense stop codons UAA, UAG or UGA can change their effect and makes it impossible for the ribosome to recognize the stop codon, hence translation continues with 
incorporation of the corresponding tRNA (Hoernes et al., 2016; Karijolich and $\mathrm{Yu}, 2011)$. An alternative way to regulate translation was shown by in vitro and in vivo assays in which the pseudouridylated mRNA had an increased translation capacity as well as higher biological stability in mice and humans (Kariko et al., 2008). Altered pseudouridylation levels in humans have been shown to cause intellectual disability and microcephaly (Shaheen et al., 2019).

\subsubsection{5 $\quad N^{4}$-acetylcytidine modification}

Very recently, the ac4C modification has been identified in yeast and has also been mapped in human mRNAs (Arango et al., 2018; Tardu et al., 2018). The ac4C modification is installed by NAT10 in a broad range of human mRNAs in addition to $18 \mathrm{~S}$ rRNA, Serine and Leucine tRNAs (Arango et al., 2018; Chimnaronk et al., 2009; Ito et al., 2014; Sharma et al., 2015). Over 4,000 ac4C marks were identified within human mRNAs with usually one or two ac4C modified nucleosides per modified transcript. Positional analysis of ac4C revealed a uniform distribution all over the mRNAs with a general bias towards the $5^{\prime}$ UTR close to translation start sites (Arango et al., 2018). Depletion of NAT10 caused downregulated expression levels of ac4C containing mRNA indicating a positive effect of the ac4C modification on translation efficiency (Arango et al., 2018). Additionally, the mRNA stability of ac4C containing transcripts was significantly prolonged compared to mRNAs in NAT10 deficient cells (Arango et al., 2018). Moreover, mRNA decay and translation are intricately linked. Thus, decreased mRNA stability results in a reduced translation, what decreases mRNA stability reciprocally (Hanson and Coller, 2018). In addition to the elevated translation efficiency of ac4C containing mRNAs, ac4C in the wobble position of tRNAs also stimulates translation (Arango et al., 2018). 
2.3.4 $N^{3}$-methylcytidine modification

The presence of a $N^{3}$-methylcytidine (m3C) (Figure 6) modification was observed in tRNAs Serine and Threonine at position C32 in the model organism S. cerevisiae (D'Silva et al., 2011; Noma et al., 2011) and humans (Clark et al., 2016). The variable arm of tRNA Serine and C32 in tRNA Arginine with anticodon $\mathrm{CCU}$ and UCU also contain $\mathrm{m} 3 \mathrm{C}$ in higher eukaryotes (Arimbasseri et al., 2015; Capone et al., 1985; Cribbs et al., 1987; Keith, 1984; Partial et al., 1971).

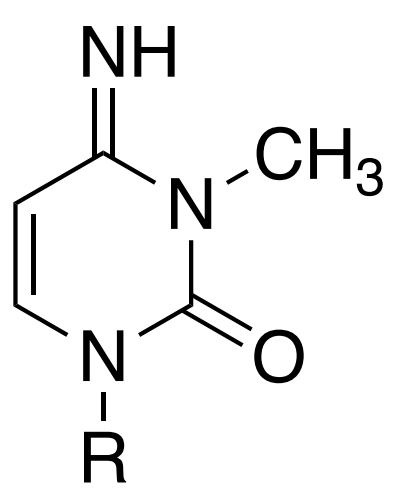

Figure 6: Structure of the $N^{3}$-methylcytosine (m3C) modification.

The additional methyl group is added to the $\mathrm{N}-3$ of cytidine by the methyltransferase Trm140 in yeast and METTL2, 6 or 8 in humans and S-adenosylmethionine (SAM).

\subsubsection{1 $\quad N^{3}$-methylcytidine methyltransferase in S. cerevisiae}

The methyltransferase for m3C is Trm140 in S. cerevisiae (D'Silva et al., 2011; Noma et al., 2011). Trm140 was first identified as an actin binding protein (Abp140) (Asakura et al., 1998) with its $\mathrm{N}$-terminal part binding to actin filaments and thereby localizing to actin patches and cables. The C-terminal part is responsible for $\mathrm{m} 3 \mathrm{C}$ modification and contains the $\mathrm{S}$ adenosylmethionine (SAM) binding motif. Trm140 is encoded on two separate open reading frames (ORFs) that are translated into one protein by $a+1$ frame shift (Farabaugh et al., 2006). It seems likely that the $\mathrm{m} 3 \mathrm{C}$ modification on position C32 of tRNA is important for cells, as residue 32 interacts with residue 38 of the anticodon loop to maintain its structure (Auffinger and Westhof, 2001, 
1999). A Trm140 knockout (KO) does not have a significant phenotype, whereas the Trm140 and Trm1 double knockout strain, which lacks $\mathrm{m} 3 \mathrm{C}$ and $N^{2}-N^{2}$-dimethylguanine $\left(\mathrm{m}^{2}{ }_{2} \mathrm{G}\right)$, is sensitive to cycloheximide, indicating impaired translocation on the ribosome (D'Silva et al., 2011). $N^{6}$-isopentenyl adenosine (i6A37), $N^{6}$-threonylcarbamoyl adenosine (t6A37) or their derivatives at position 37 are necessary for $\mathrm{m} 3 \mathrm{C}$ modification at position 32 (Han et al., 2017). However, the t6A37 is not a sufficient mark for m3C modification as shown for several tRNAs (Met, lle, Asn Lys) (Thiaville et al., 2016). It has been shown that Trm140 recognizes its tRNA substrates in two different ways. It can either recognize the sequence G35-U36-t6A37 of the anticodon loop of tRNA Threonine or t6A37 and i6A37 of tRNA Serine, since the anticodons of tRNA Serine do not have G35-U36 and no other nucleotides in common. Additionally, seryl-tRNA synthetase and the distinctive tRNA Serine variable loop are important to achieve Trm140 specificity on tRNA Serine (Han et al., 2017).

\subsubsection{2 $\quad N^{3}$-methylcytidine methyltransferases in higher eukaryotes}

In mammals, several methyltransferase-like (METTL) proteins have been well characterized and a high sequence similarity of the human and mouse METTL2, METTL6 and METTL8 to the yeast Trm140 was shown (Xu et al., 2017). METTL2 and METTL6 contribute to specific tRNA modification, whereas METTL8 forms m3C in mRNAs (Xu et al., 2017). A knockout of METTL8 in two different human cell lines drastically reduced the $\mathrm{m} 3 \mathrm{C}$ level in mRNAs, suggesting that METTL8 is the only methyltransferase for $\mathrm{m} 3 \mathrm{C}$ in mRNAs (Xu et al., 2017). The overall presence of m3C in mRNAs has been shown by HPLC-coupled triple quadrupole mass spectrometry (LC-MS/MS). Thereby, a similar abundance of $\mathrm{m} 3 \mathrm{C}\left(5\right.$ per $\left.10^{5} \mathrm{C}\right)$ as $\mathrm{m} 1 \mathrm{~A}\left(7\right.$ per $\left.10^{5} \mathrm{~A}\right)$ and m5C (9 per $\left.10^{5} \mathrm{C}\right)$ and lower levels than $\mathrm{m} 6 \mathrm{~A}\left(1-2\right.$ per $\left.10^{3} \mathrm{~A}\right)$ have been found (Xu et al., 2017). However, NGS sequencing and thus genome wide information about its localization on transcripts is still missing. Localization on mRNA transcripts in the $5^{\prime}$ UTR, the coding region or the $3^{\prime}$ UTR may shed light on the functional implication of the $\mathrm{m} 3 \mathrm{C}$ modification. 


\subsection{Aims of this thesis}

2.4.1 Transcriptome maps of general eukaryotic RNA degradation factors

A large variety of different RNA degradation factors poses the question how RNA degradation pathways are selected and how the RNA sequence encodes or influences this selection. Answering this question requires a systematic analysis of the RNA-binding profiles of the involved protein factors. Although several transcriptome profiles of the RNA degradation factors $5^{\prime} \rightarrow 3^{\prime}$ exonuclease Xrn1, exosome subunits (Rrp44, Csl4, Rrp41, and Rrp6), TRAMP subunits (Mtr4, Trf4, and Air2) and Ski complex subunit Ski2 have been reported (Delan-Forino et al., 2017; Milligan et al., 2016; Schneider et al., 2012; Tuck and Tollervey, 2013), there is a lack of transcriptome-wide binding profiles for components of the deadenylation, decapping, and NMD machineries, as well as subunits of the exosome complex and exosome associated factors. Thus, the task of systematically analyzing the binding of subunits from many known factors involved in RNA degradation to a eukaryotic transcriptome ('transcriptome mapping') has not been accomplished yet. I will use the established method photoactivatable ribonucleoside-enhanced crosslinking and immunoprecipitation (PAR-CLIP) for 30 general RNA degradation factors to systematically generate transcriptome-wide protein binding profiles in the yeast S. cerevisiae. In combination with thorough bioinformatic analyses we will draw conclusions regarding protein complex interactions within degradation pathways.

2.4.2 Identification and functional characterization of the novel mRNA modification $N^{3}$-methylcytidine

The m3C modification has so far only been observed in tRNAs of yeast and human cells and in human mRNAs (D'Silva et al., 2011; Noma et al., 2011; $\mathrm{Xu}$ et al., 2017). However, the presence in yeast mRNA and the positional information regarding the localization within human mRNA transcripts is still lacking. To investigate and localize the $\mathrm{m} 3 \mathrm{C}$ modification in mRNAs of S. cerevisiae, I will perform PAR-CLIP experiments of Trm140 to analyze its 
mRNA binding ability. Protein binding to mRNA suggests a modification in proximity to the binding site. I will use this approach in combination with a newly developed method using anti-m3C antibodies for RNA immunoprecipitation, called m3C-CLIP. Functional analyses by metabolic labeling and sequencing and analyses of ribosome profiling data in yeast will be used to elucidate a potential function of the $\mathrm{m} 3 \mathrm{C}$ modification regarding RNA stability and translational fidelity. In addition to the work in yeast, I will perform PAR-CLIP experiments of METTL8 and m3C-CLIP in human HEK293 cells to elucidate the localization of the $\mathrm{m} 3 \mathrm{C}$ modification on protein coding transcripts. 


\section{Materials and Methodology}

\subsection{Materials}

\subsubsection{Bacterial strains}

Table 1: Bacterial strains used in this study.

\begin{tabular}{|c|c|c|}
\hline Strain & Genotype & Source \\
\hline $\begin{array}{l}\text { BL21-Codon } \\
\text { Plus(DE3)-RIL }\end{array}$ & $\begin{array}{l}\text { recA1 endA1 gyrA96 thi-1 hsdR17 } \\
\text { Stratagene supE44 relA1 lac [F' } \\
\left.\text { proAB lacl } \mathrm{q} \text { Z } \Delta \mathrm{M} 15 \mathrm{Tn} 10\left(\mathrm{Tet}^{\mathrm{r}}\right)\right]\end{array}$ & Stratagene \\
\hline XL1-Blue & $\begin{array}{l}\text { recA1 endA1 gyrA96 thi-1 hsdR17 } \\
\text { Stratagene supE44 relA1 lac [F' } \\
\left.\text { proAB lacl' } \mathrm{q} \text { ZMM15 Tn10 }\left(\mathrm{Tet}^{\mathrm{r}}\right)\right]\end{array}$ & Stratagene \\
\hline
\end{tabular}

3.1.2 Yeast strains

Table 2: Yeast strains used in this study.

\begin{tabular}{|c|c|c|}
\hline Strain & Genotype & Source \\
\hline BY4741 (Wildtype) & $\begin{array}{l}\text { MATa; his3 } \Delta 1 ; \text { leu2 } \Delta 0 ; \text { met15 } \Delta 0 \text {; } \\
\text { ura3 } \Delta 0\end{array}$ & Euroscarf \\
\hline Air1-TAP & BY4741; AIR1-TAP::HIS3MX6 & Euroscarf \\
\hline Air2-TAP & BY4741; AIR2-TAP::HIS3MX6 & Euroscarf \\
\hline Caf40-TAP & BY4741; CAF40-TAP::HIS3MX6 & Euroscarf \\
\hline Ccr4-TAP & BY4741; CCR4-TAP::HIS3MX6 & Euroscarf \\
\hline CsI4-TAP & BY4741; CSL4-TAP::HIS3MX6 & Euroscarf \\
\hline Dcp1-TAP & BY4741; DCP1-TAP::HIS3MX6 & Euroscarf \\
\hline Dcp2-TAP & BY4741; DCP2-TAP::HIS3MX6 & Euroscarf \\
\hline Dhh1-TAP & BY4741; DHH1-TAP::HIS3MX6 & Euroscarf \\
\hline Edc2-TAP & BY4741; EDC2-TAP::HIS3MX6 & Euroscarf \\
\hline Edc3-TAP & BY4741; EDC3-TAP::HIS3MX6 & Euroscarf \\
\hline Mtr4-TAP & BY4741; MTR4-TAP::HIS3MX6 & Euroscarf \\
\hline Nmd4-TAP & BY4741; NMD4-TAP::HIS3MX6 & Euroscarf \\
\hline Not1-TAP & BY4741; NOT1-TAP::HIS3MX6 & Euroscarf \\
\hline
\end{tabular}




\begin{tabular}{|l|l|l|}
\hline Pan2-TAP & BY4741; PAN2-TAP::HIS3MX6 & Euroscarf \\
\hline Pan3-TAP & BY4741; PAN3-TAP::HIS3MX6 & Euroscarf \\
\hline Pop2-TAP & BY4741; POP2-TAP::HIS3MX6 & Euroscarf \\
\hline Rrp4-TAP & BY4741; RRP4-TAP::HIS3MX6 & Euroscarf \\
\hline Rrp6-TAP & BY4741; RRP6-TAP::HIS3MX6 & Euroscarf \\
\hline Rrp40-TAP & BY4741; RRP40-TAP::HIS3MX6 & Euroscarf \\
\hline Rrp44-TAP & BY4741; RRP44-TAP::HIS3MX6 & Euroscarf \\
\hline Ski2-TAP & BY4741; SKI2-TAP::HIS3MX6 & Euroscarf \\
\hline Ski3-TAP & BY4741; SKI3-TAP::HIS3MX6 & Euroscarf \\
\hline Ski7-TAP & BY4741; SKI7-TAP::HIS3MX6 & Euroscarf \\
\hline Ski8-TAP & BY4741; SKI8-TAP::HIS3MX6 & Euroscarf \\
\hline Trf4-TAp & BY4741; TRF4-TAP::HIS3MX6 & Euroscarf \\
\hline Trf5-TAP & BY4741; TRF5-TAP::HIS3MX6 & Euroscarf \\
\hline Trm140-TAP & BY4741; BUR1-TAP::HIS3MX6 & Euroscarf \\
\hline Trm140 knockout & $\begin{array}{l}\text { BY4741; MATa; ura3 } \triangle 0 ; \text { leu2 } 20 ; \\
\text { his3 } 1 ; \text { met15 } 0 ; \\
\text { YOR239w::kanMX4 }\end{array}$ & Euroscarf \\
\hline Upf1-TAP & BY4741; UPF1-TAP::HIS3MX6 & Euroscarf \\
\hline Upf2-TAP & BY4741; UPF2-TAP::HIS3MX6 & Euroscarf \\
\hline Upf3-TAP & BY4741; UPF3-TAP::HIS3MX6 & Euroscarf \\
\hline Xrn1-TAP & BY4741; XRN1-TAP::HIS3MX6 & Euroscarf \\
\hline
\end{tabular}

\subsubsection{Human cell lines}

Table 3: Human cell lines used in this study.

\begin{tabular}{|l|l|}
\hline Cell line & Description \\
\hline Flp-In ${ }^{\mathrm{TM}}$ T-REx TM $^{\mathrm{T}}$ 293 Cell Line & Modified HEK293 cell line used for \\
(Thermo Fisher Scientific) & generation of a stable cell line of \\
& METTL8-3xFLAG from a FIp-In ${ }^{\mathrm{TM}}$ \\
& expression vector by homologous \\
& recombination. \\
\hline
\end{tabular}




\subsubsection{Media and supplement}

Table 4: Growth media used in this study.

\begin{tabular}{|l|l|l|}
\hline Name & Description/ source & Species \\
\hline Lysogeny Broth (LB) & $\begin{array}{l}1 \%(w / v) \text { tryptone; 0.5\% } \\
(w / v) \text { yeast extract; 0.5\% } \\
(w / v) \text { NaCl; }(+1.5 \%(w / v) \\
\text { agar for solid media plates) }\end{array}$ & E. coli \\
\hline $\begin{array}{l}\text { Yeast extract peptone } \\
\text { dextrose (YPD) }\end{array}$ & $\begin{array}{l}\text { glucose; } 1.5 \%(w / v) \text { yeast } \\
\text { extract }(+1.8 \%(w / v) \text { agar for } \\
\text { solid media plates) }\end{array}$ & S. cerevisiae \\
\hline $\begin{array}{l}\text { Synthetic complete } \\
\text { medium (SCM) }\end{array}$ & $\begin{array}{l}0.69 \%(w / v) \text { yeast nitrogen } \\
\text { base without amino acids; } \\
0.04 \%(w / v) \text { complete } \\
\text { supplement mixture; 0.04\% } \\
(w / v) \text { complete supplement } \\
\text { mixture without uracil } \\
\text { (Formedium) }\end{array}$ & S. cerevisiae \\
\hline $\begin{array}{l}\text { Dulbecco's Modified } \\
\text { Eagle Medium } \\
\text { (DMEM) }\end{array}$ & $\begin{array}{l}\text { Growth and maintenance } \\
\text { medium from Thermo Fisher } \\
\text { Scientific }\end{array}$ & H. sapiens \\
\hline
\end{tabular}

Table 5: Media supplements used in this study.

\begin{tabular}{|l|l|l|}
\hline \hline Name & Description/ source & $\begin{array}{l}\text { Working } \\
\text { concentation }\end{array}$ \\
\hline 4-thiouracil & $\begin{array}{l}\text { Labeling of nascent RNA (S. } \\
\text { cerevisiae) from Sigma }\end{array}$ & $1 \mathrm{mM}$ \\
\hline 4-thiouridine & $\begin{array}{l}\text { Labeling of nascent RNA } \\
\text { (human) from Carbosynth }\end{array}$ & $500 \mu \mathrm{M}$ \\
\hline Blasticidin $\mathrm{HCl}$ & $\begin{array}{l}\text { Antibiotic from Thermo } \\
\text { Fisher Scientific }\end{array}$ & $15 \mu \mathrm{g} / \mathrm{mL}$ \\
\hline FBS & $\begin{array}{l}\text { Fetal bovine serum as } \\
\text { supplement for DMEM from } \\
\text { Thermo Fisher Scientific }\end{array}$ & $1: 10$ \\
\hline GlutaMAX (200 mM) & $\begin{array}{l}\text { Supplement for DMEM from } \\
\text { Thermo Fisher Scientific }\end{array}$ & $1: 100$ \\
\hline Hygromycin & Antibiotic from Invitrogen & $50 \mu \mathrm{g} / \mathrm{mL}$ \\
\hline
\end{tabular}




\begin{tabular}{|l|l|l|}
\hline $\begin{array}{l}\text { Isopropyl- } \beta \text { - D- } \\
\text { thiogalactopyranosid } \\
\text { (IPTG) }\end{array}$ & $\begin{array}{l}\text { Gene experession induction } \\
\text { in E. coli from Carl Roth }\end{array}$ & $1 \mathrm{mM}$ \\
\hline Kanamycin & Antibiotic from Carl Roth & $50 \mu \mathrm{g} / \mathrm{mL}$ \\
\hline Tetracycline & $\begin{array}{l}\text { Gene of interest expression } \\
\text { induction in the Flp-In } \\
\text { RE T- } \\
\text { REx } \\
\text { Thermo Fisher Scientific. }\end{array}$ & $1 \mu \mathrm{M}$ \\
\hline Zeocin & Antibiotic from Invitrogen & $100 \mu \mathrm{g} / \mathrm{mL}$ \\
\hline
\end{tabular}

\subsubsection{Spike-ins}

Table 6: Spike-ins used in this study provided by Dr. Kristina Zumer.

\begin{tabular}{|l|l|l|l|l|l|}
\hline Spike-in & ERCC-ID & length & $\begin{array}{l}\text { Number } \\
\text { of U }\end{array}$ & $\begin{array}{l}\text { GC content } \\
(\%)\end{array}$ & $\begin{array}{l}\text { 4sU } \\
\text { labeled }\end{array}$ \\
\hline Spike 2 & $\begin{array}{l}\text { Derived from } \\
\text { ERCC-00043 }\end{array}$ & 985 & 297 & 33.9 & yes \\
\hline Spike 4 & $\begin{array}{l}\text { Derived from } \\
\text { ERCC-00136 }\end{array}$ & 1014 & 268 & 42.6 & yes \\
\hline Spike 5 & $\begin{array}{l}\text { Derived from } \\
\text { ERCC-00145 }\end{array}$ & 1015 & 264 & 42.8 & no \\
\hline Spike 8 & $\begin{array}{l}\text { Derived from } \\
\text { ERCC-00092 }\end{array}$ & 1079 & 287 & 51.8 & yes \\
\hline Spike 9 & $\begin{array}{l}\text { Derived from } \\
\text { ERCC-00002 }\end{array}$ & 1037 & 263 & 52.8 & no \\
\hline Spike 12 & $\begin{array}{l}\text { Derived from } \\
\text { ERCC-00170 }\end{array}$ & 949 & 296 & 34.8 & no \\
\hline
\end{tabular}

\subsubsection{Primers and oligonucleotides}

Table 7: Primers and oligonucleotides used in this study were purchased from IDT.

\begin{tabular}{|l|l|}
\hline \hline Name & Sequence \\
\hline $3^{\prime}$ adapter & /5rApp/TGGAA TTCTCGGGTGCCAAGG/3ddC/ \\
\hline 5' $^{\prime}$ adapter & $\begin{array}{l}\text { /5InvddT/rGrUrUrCrArGrArGrUrUrCrUrArCrArGrUrCr } \\
\text { CrGrArCrGrArUrCrNrNrNrNrN }\end{array}$ \\
\hline RT primer & CCTTGGCACCCGAGAATTCCA \\
\hline
\end{tabular}




\begin{tabular}{|l|l|}
\hline $\begin{array}{l}\text { NEXTflex barcode } \\
\text { primer }\end{array}$ & $\begin{array}{l}\text { CAAGCAGAAGACGGCATACGAGA-Barcode- } \\
\text { GTGACTGGAGTTC CTTGGCACCCGAGAA TTCCA }\end{array}$ \\
\hline Barcode 1 & ATCACG \\
\hline Barcode 2 & CGATGT \\
\hline Barcode 3 & TTAGGC \\
\hline Barcode 4 & TGACCA \\
\hline Barcode 5 & ACAGTG \\
\hline Barcode 6 & GCCAAT \\
\hline Barcode 7 & CAGATC \\
\hline Barcode 8 & ACTTGA \\
\hline Barcode 9 & GATCAG \\
\hline Barcode 10 & TAGCTT \\
\hline Universal primer & AATGATACGGCGACCACCGAGATCTACACGTTC \\
AGAGTTCTACAGTCCGA \\
\hline Nextera primer 1 & AATGATACGGCGACCACCGA \\
\hline Nextera primer 2 & CAAGCAGAAGACGGCATACGA \\
\hline RT primer Serine & FAM-CGACACCAGCAGGATTTGAA \\
\hline RT primer Arginine & FAM-CGTTCCGTACGGGACT \\
\hline
\end{tabular}

3.1.7 Thermal cycler programs

Table 8: List of thermal cycler programs used in this study.

\begin{tabular}{|l|l|l|l|l|}
\hline \hline Program & Step & Temperature $/{ }^{\circ} \mathrm{C}$ & Time $/$ sec & Repeat \\
\hline \hline Fusion PCR & 1 & 98 & 120 & \\
\cline { 2 - 5 } & 2 & 98 & 20 & \\
\cline { 2 - 5 } & 3 & 60 & 30 & \\
\cline { 2 - 5 } & 4 & 72 & 30 & $\begin{array}{l}\text { Repeat steps } \\
\text { 2-4 29 times }\end{array}$ \\
\cline { 2 - 5 } & 5 & 72 & 300 & \\
\hline One-step PCR & 1 & 95 & 300 & \\
\cline { 2 - 5 } & 2 & 98 & 80 & \\
\cline { 2 - 5 } & 3 & 55 & 30 & \\
\cline { 2 - 5 } & 4 & 72 & 60 & \\
\cline { 2 - 5 } & 5 & 72 & 180 & \\
\hline
\end{tabular}


3.1.8 Plasmids

Table 9: List of plasmids used in this study.

\begin{tabular}{|l|l|l|}
\hline \hline Vector & Description & Source \\
\hline \hline 438-C & $\begin{array}{l}\text { N-terminal His 6x tag, MBP tag, N10 } \\
\text { linker and a TEV protease cleavage site }\end{array}$ & UC Berkeley \\
\hline pcDNA $^{\text {TM5/FRT }}$ & $\begin{array}{l}\text { Expression vector into which the gene of } \\
\text { interest was cloned }\end{array}$ & $\begin{array}{l}\text { Thermo Fisher } \\
\text { Scientific }\end{array}$ \\
\hline pOG44 & $\begin{array}{l}\text { Flp recombinase expression plasmid } \\
\text { under the control of the human CMV } \\
\text { promoter. }\end{array}$ & $\begin{array}{l}\text { Thermo Fisher } \\
\text { Scientific }\end{array}$ \\
\hline
\end{tabular}

3.1.9 Buffers and solutions

Table 10: List of buffers and solutions used in this study.

\begin{tabular}{|c|c|}
\hline Name & Ingredients \\
\hline $\begin{array}{l}\text { Lysis buffer } \\
\text { (yeast) }\end{array}$ & $\begin{array}{l}50 \mathrm{~mm} \text { Tris- } \mathrm{HCl} \mathrm{pH} 7.5,100 \mathrm{~mm} \mathrm{NaCl}, 0.1 \% \text { SDS, } 0.5 \% \\
\text { NP-40, 0.5\% Na-deoxycholate }\end{array}$ \\
\hline $\begin{array}{l}\text { Lysis buffer } \\
\text { (human) }\end{array}$ & $\begin{array}{l}50 \mathrm{~mm} \text { HEPES-KOH pH 7.5, } 150 \mathrm{~mm} \mathrm{KCl,} 2 \mathrm{~mm} \text { EDTA- } \\
\mathrm{NaOH}, \mathrm{pH} 8.0,1 \mathrm{mM} \mathrm{NaF}, 0.5 \%(\mathrm{v} / \mathrm{v}) \mathrm{NP} 40 \text { substitute, } \\
0.5 \mathrm{~mm} \text { DTT, complete EDTA-free protease inhibitor } \\
\text { cocktail }\end{array}$ \\
\hline $\begin{array}{l}\text { Wash buffer } \\
\text { (yeast) }\end{array}$ & $\begin{array}{l}50 \mathrm{mM} \text { Tris-HCl pH 7.5, } 1 \mathrm{M} \mathrm{NaCl}, 0.1 \% \text { SDS, } 0.5 \% \text { NP-40, } \\
0.5 \% \text { Na-deoxycholate }\end{array}$ \\
\hline T1 buffer & $50 \mathrm{~mm}$ Tris-HCl pH 7.5, 2 mm EDTA \\
\hline $\begin{array}{l}\text { High salt wash } \\
\text { buffer }\end{array}$ & $\begin{array}{l}50 \mathrm{mM} \text { HEPES-KOH pH 7.5, } 500 \mathrm{~mm} \mathrm{KCl,} \mathrm{0.05 \%}(\mathrm{v} / \mathrm{v}) \text {, } \\
\text { NP40 substitute, } 0.5 \mathrm{~mm} \text { DTT, complete EDTA-free } \\
\text { protease inhibitor cocktail }\end{array}$ \\
\hline $\begin{array}{l}\text { Phosphatase } \\
\text { buffer }\end{array}$ & $50 \mathrm{~mm}$ Tris- $\mathrm{HCl} \mathrm{pH} \mathrm{7.0,1} \mathrm{mM} \mathrm{MgCl} 2,0.1 \mathrm{mM} \mathrm{ZnCl} 2$ \\
\hline $\begin{array}{l}\text { Phosphatase } \\
\text { wash buffer }\end{array}$ & $\begin{array}{l}50 \mathrm{~mm} \text { Tris- } \mathrm{HCl} \mathrm{pH} \mathrm{7.5,} 20 \mathrm{~mm} \text { EGTA, 0.5\% (v/v) NP40 } \\
\text { substitute }\end{array}$ \\
\hline PNK buffer & $50 \mathrm{~mm}$ Tris- $\mathrm{HCl} \mathrm{pH} 7.5,50 \mathrm{~mm} \mathrm{NaCl}, 10 \mathrm{~mm} \mathrm{MgCl} 2$ \\
\hline $\begin{array}{l}\text { Proteinase } \mathrm{K} \\
\text { buffer }\end{array}$ & $\begin{array}{l}50 \text { mM Tris- } \mathrm{HCl} \mathrm{pH} 7.5,6.25 \mathrm{~mm} \text { EDTA, } 75 \mathrm{~mm} \mathrm{NaCl}, 1 \% \\
(v / v) \text { SDS }\end{array}$ \\
\hline Buffer A & $\begin{array}{l}300 \mathrm{~mm} \mathrm{NaCl}, 20 \mathrm{~mm} \text { Na-HEPES pH } 7.4,30 \mathrm{~mm} \text { imidazole } \\
1 \mathrm{mM} \text { DTT, } 10 \% \text { glycerol, } 0.284 \mathrm{mg} / \mathrm{mL} \text { leupeptin, } \\
1.37 \mathrm{mg} / \mathrm{mL} \text { pepstatin A, } 0.17 \mathrm{mg} / \mathrm{mL} \text { PMSF, } 0.33 \mathrm{mg} / \mathrm{mL} \\
\text { benzamidine }\end{array}$ \\
\hline
\end{tabular}




\begin{tabular}{|c|c|}
\hline Buffer B & $\begin{array}{l}1 \mathrm{~m} \mathrm{NaCl}, 20 \mathrm{~mm} \mathrm{Na} \text {-HEPES pH } 7.4,30 \mathrm{~mm} \text { imidazole, } \\
1 \mathrm{mM} \mathrm{DTT}, 10 \%(\mathrm{v} / \mathrm{v}) \text { glycerol, } 0.284 \mathrm{mg} / \mathrm{mL} \text { leupeptin, } \\
1.37 \mathrm{mg} / \mathrm{mL} \text { pepstatin A, } 0.17 \mathrm{mg} / \mathrm{mL} \text { PMSF, } 0.33 \mathrm{mg} / \mathrm{mL} \\
\text { benzamidine }\end{array}$ \\
\hline Buffer C & $\begin{array}{l}300 \mathrm{~mm} \mathrm{NaCl}, 20 \mathrm{mM} \text { Na-HEPES pH 7.4, } 500 \mathrm{mM} \\
\text { imidazole, } 1 \mathrm{mM} \text { DTT, } 10 \% \text { (v/v) glycerol, } 0.284 \mathrm{mg} / \mathrm{mL} \\
\text { leupeptin, } 1.37 \mathrm{mg} / \mathrm{mL} \text { pepstatin A, } 0.17 \mathrm{mg} / \mathrm{mL} \text { PMSF, } \\
0.33 \mathrm{mg} / \mathrm{mL} \text { benzamidine }\end{array}$ \\
\hline Buffer D & $\begin{array}{l}300 \mathrm{~mm} \text { NaCl, } 20 \mathrm{~mm} \text { Na-HEPES pH 7.4, } 30 \mathrm{~mm} \text { imidazole, } \\
1 \mathrm{~mm} \text { DTT, } 10 \%(\mathrm{v} / \mathrm{v}) \text { glycerol }\end{array}$ \\
\hline Buffer E & $\begin{array}{l}300 \mathrm{~mm} \mathrm{NaCl}, 20 \mathrm{~mm} \text { Na-HEPES pH 7.4, } 1 \text { mM DTT, } 10 \% \\
(\mathrm{v} / \mathrm{v}) \text { glycerol }\end{array}$ \\
\hline
\end{tabular}

\subsubsection{Antibodies}

Table 11: List of antibodies used in this study.

\begin{tabular}{|l|l|l|l|}
\hline Name & Dilution & Application & Source \\
\hline Anti-m3C & $30 \mu \mathrm{L}$ per IP & m3C-CLIP & Active Motif \\
\hline $\begin{array}{l}\text { Anti-FLAG M2 magnetic } \\
\text { beads }\end{array}$ & $\begin{array}{l}100 \mu \mathrm{L} \text { per } \\
\text { IP }\end{array}$ & PAR-CLIP & Sigma Aldrich \\
\hline Anti-FLAG M2 & $1: 1000$ & WB & Sigma Aldrich \\
\hline Anti-METTL8 & $1: 1000$ & WB & $\begin{array}{l}\text { Thermo Fisher } \\
\text { Scientific }\end{array}$ \\
\hline Anti-mouse-HRP & $1: 3000$ & WB & Abcam \\
\hline Anti-rabbit-HRP & $1: 5000$ & WB & GE Healthcare \\
\hline Anti-rat-HRP & $1: 5000$ & WB & Sigma Aldrich \\
\hline IgG from rabbit serum & $\begin{array}{l}100 \mu \mathrm{g} \text { per } \\
\text { IP }\end{array}$ & PAR-CLIP & Sigma \\
\hline
\end{tabular}




\subsection{Experimental methodology}

\subsubsection{Yeast culture}

S. cerevisiae cells expressing the TAP-tagged protein were cultured from $\mathrm{OD}_{600} \sim 0.1$ to $\mathrm{OD}_{600} \sim 0.5$ in $\mathrm{SCM}$ minimal medium (Formedium) supplemented with $89 \mu \mathrm{M}$ uracil, $100 \mu \mathrm{M}$ 4-thiouracil (4tU), and $2 \%$ glucose at $30{ }^{\circ} \mathrm{C}$. After reaching $\mathrm{OD}_{600} \sim 0.5,4 \mathrm{tU}$ was added to a final concentration of 1 $\mathrm{mM}$, and cells were cultured further for $4 \mathrm{~h}$ (final $\mathrm{OD}_{600} \sim 1.3-1.6$ ).

\subsubsection{S. cerevisiae strain validation by SDS-PAGE and Western Blot}

S. cerevisiae BY4741 strains containing tagged genes were tested for expression of the specific $\mathrm{C}$-terminally tandem affinity purification (TAP)-tagged (Euroscarf) protein by Western blotting. Cells were lysed and the diluted lysate was subjected to a precast $4-12 \%$ NuPAGE Bis-Tris gel (Invitrogen) for gel electrophoresis. Following SDS-PAGE, samples were transferred onto a PVDF membrane (Bio-Rad). Subsequently, the membrane was incubated with a primary antibody coupled to horseradish peroxidase (HRP) against the TAP tag (PAP; Sigma-Aldrich). The antibody was detected using Pierce enhanced chemiluminescence (ECL) Western blotting substrate (Thermo Fisher Scientific) and a ChemoCam imager (Intas).

3.2.3 Generation of the METTL8-3xFLAG Flp-In ${ }^{\text {TM }}$ T-REx ${ }^{\text {TM }} 293$ Cell Line

For the generation of a stable cell line expression METTL8 C-terminally 3XFLAG tagged, the coding sequence was cloned into a pcDNA5 vector including the 3xFLAG sequence (DYKDDDDK). HEK293 stable cell lines expressing METTL8-3xFlag were generated using the Flp-In ${ }^{\mathrm{TM}}{ }^{\mathrm{T}}$-REx ${ }^{\mathrm{TM}}$ system (Thermo Fisher Scientific) according to the manufacturer's instructions. HEK293 FIp-In TM T-REX ${ }^{T M}$ cells were cultured in Dulbecco's modified Eagle's medium (DMEM) supplemented with $10 \%$ fetal bovine serum (FBS) and $1 \mathrm{X}$ GlutaMAX (Thermo Fisher Scientific) at $37^{\circ} \mathrm{C}$ with $5 \% \mathrm{CO}_{2}$. 


\subsubsection{PAR-CLIP}

\subsubsection{S. cerevisiae}

PAR-CLIP and data acquisition were performed as described (Baejen et al., 2017, 2014; Battaglia et al., 2017; Schulz et al., 2013) with minor modifications. Yeast cells harboring the TAP-tagged protein were grown in minimal medium (CSM mixture, Formedium) containing $89 \mu \mathrm{M}$ uracil, $100 \mu \mathrm{M} 4-$ thiouracil ( $4 \mathrm{tU}$ ) and $2 \%$ glucose from $\mathrm{OD}_{600} \sim 0.1$ to $\sim 0.5$ at $30^{\circ} \mathrm{C}$. After addition of $4 \mathrm{tU}$ to a final concentration of $1 \mathrm{~mm}$, cells were grown further for $4 \mathrm{~h}$. $4 \mathrm{tU}-$ labeled yeast cells were collected and resuspended in $10 \mathrm{~mL}$ PBS for UVirradiation with an energy dose of $12 \mathrm{~J} / \mathrm{cm}^{2}$ at $365 \mathrm{~nm}$ on ice and constant shaking. Harvested cells were resuspend in lysis buffer and lysed by bead beating (FastPrep-24 Instrument, MP Biomedicals) with $1 \mathrm{~mL}$ silica-zirconium beads (Roth) with $8 \times 40 \mathrm{sec}$ repeats at $4 \mathrm{~m} / \mathrm{s}$ and with $1 \mathrm{~min}$ incubation on ice between each step. Immunoprecipitation was performed on a rotating wheel over night at $4{ }^{\circ} \mathrm{C}$ with rabbit IgG-conjugated protein $\mathrm{G}$ magnetic Dynabeads (Invitrogen) using $330 \mu \mathrm{L}$ beads and $100 \mu \mathrm{g}$ antibody. Beads were washed twice in $1 \mathrm{~mL}$ wash buffer and twice in $1 \mathrm{~mL} \mathrm{T1}$ buffer. A partial digest of the crosslinked RNA was performed with $20 \mathrm{U}$ RNase T1 in $400 \mu \mathrm{L}$ T1 buffer for $25 \mathrm{~min}$ at $25^{\circ} \mathrm{C}$. To stop the RNase reaction, the sample was immediately cooled on ice for $5 \mathrm{~min}$. To remove spare RNase T1, beads were washed twice in T1 buffer and phosphatase buffer. Dephosphorylation was performed with antarctic phosohatase (NEB) and $1 \mathrm{U} / \mu \mathrm{L}$ RNase OUT (Invitrogen) for $30 \mathrm{~min}$ at $37^{\circ} \mathrm{C}$. Beads were washed once in phosphatase wash buffer and twice in polynucleotide kinase (PNK) buffer. Phosphorylation was performed in T4 PNK reaction buffer $A$ (Fermentas) with final concentration of $1 \mathrm{U} / \mu \mathrm{L}$ T4 PNK, $1 \mathrm{U} / \mu \mathrm{L}$ RNase OUT and $1 \mathrm{~mm}$ ATP (cold labeling) or $0.1 \mu \mathrm{Ci}$ gamma-32-P-ATP (Perkin Elmer) (radioactive labeling) for Trm140 PAR-CLIP. The reaction was incubated at $37^{\circ} \mathrm{C}$ for $1 \mathrm{~h}$. For the radioactive labeling reaction mix, the sample was subsequently spiked with $1 \mathrm{mM} \mathrm{ATP}$ final concentration for $5 \mathrm{~min}$ at $37^{\circ} \mathrm{C}$. Beads were washed 5 times with PNK buffer and prepared for cDNA library generation. For 3' adapter ligation to the RNA, beads were incubated in T4 RNA ligase buffer (NEB) containing $10 \mathrm{U} / \mu \mathrm{L}$ T4 RNA ligase 2 (KQ) (NEB, M0373), $10 \mu \mathrm{M}$ 3' adapter (5rApp-TGGAATTCTCGGGTGCCAAGG-3ddC 
(IDT)), $1 \mathrm{U} / \mu \mathrm{L}$ RNase OUT, and 15\% (w/v) PEG 8000. The reaction was incubated for $18-20 \mathrm{~h}$ at $16{ }^{\circ} \mathrm{C}$. Beads were washed 5 times with PNK buffer to remove not ligated adapter. For $5^{\prime}$ adapter ligation to the RNA, beads were resuspended in T4 RNA ligase buffer (NEB) final concentration of $6 \mathrm{U} / \mu \mathrm{L}$ T4 RNA ligase 1 (NEB), $10 \mu \mathrm{M} \quad 5^{\prime}$ adapter (5InvddTGUUCAGAGUUCUACAGUCCGACGAUCN NNNN, IDT), $1 \mathrm{~mm}$ ATP, $1 \mathrm{U} / \mu \mathrm{L}$ RNase OUT, 5\% ( $/ v / v)$ DMSO, and 10\% (w/v) PEG 8000. The reaction was incubated for $4 \mathrm{~h}$ at $24^{\circ} \mathrm{C}$ and $1 \mathrm{~h}$ at $37^{\circ} \mathrm{C}$. Beads were washed 5 times in PNK buffer. For radioactive labeling, the sample was resuspended in $25 \mu \mathrm{L} 2 \mathrm{X}$ NuPAGE LDS Sample Buffer (Thermo Fisher Scientific) and released from the beads by incubation at $95^{\circ} \mathrm{C}$ for $5 \mathrm{~min}$. The sample was subjected to SDSPAGE on NuPAGE 4-12\% Bis-Tris Polyacrylamide gel electrophoresis (Thermo Fisher Scientific) for $1 \mathrm{~h}$ at $160 \mathrm{~V}$ in $1 \mathrm{X}$ MOPS buffer (Thermo Fisher Scientific). Radioactive RNA-protein bands were detected with the Typhoon FLA 9500 instrument (GE Healthcare Life Sciences). Electro-elution of the protein-RNA complex of interest was performed with D-Tube ${ }^{\text {TM }}$ Dialyzer Midi (Merck) according to manufacturer instructions with $800 \mu \mathrm{L}$ 1X MOPS buffer and $100 \mathrm{~V}$ for $2 \mathrm{~h}$. For cold labeling samples, the beads were incubated twice at $95{ }^{\circ} \mathrm{C}$ for $5 \mathrm{~min}$ in proteinase $\mathrm{K}$ buffer. RNA-protein complexes were subjected to protein digest using $1.5 \mathrm{mg} / \mathrm{mL}$ proteinase $\mathrm{K}(\mathrm{NEB})$ for $2 \mathrm{~h}$ at $55^{\circ} \mathrm{C}$. Acidic phenol/chloroform extraction followed by ethanol precipitation was performed in presence of $1 \mu \mathrm{L}$ GlycoBlue (Invitrogen) to recover the RNA. Reverse transcription was done for $1 \mathrm{~h}$ at $44^{\circ} \mathrm{C}$ and $1 \mathrm{~h}$ at $55^{\circ} \mathrm{C}$ using SuperScript III RTase (Invitrogen). Fusion PCR amplification was performed using the NEXTflex barcode primer kit (Bio Scientific), universal primer and Phusion HF master mix (NEB). Generated cDNA was size selected using 4\% E-Gel Agarose Gel (Invitrogen) and purified by MinElute gel extraction (Qiagen). Purified cDNA was amplified in a one-step PCR using the Nextera primers and the KAPA Library preparation kit (Roche). The library was purified using AMPure XP beads (Beckman Coulter) in a ratio of 1:1.8, subsequently quantified using TapeStation 2200 (Agilent Technologies) and Qubit (Qiagen). Samples were sequenced on an Illumina machine (HiSeq 2500 or 4000, NextSeq550). The number of independent biological replicates used for analysis is shown in Table 12. I performed 49 out of the 75 PAR-CLIP 
experiments for the degradation project. In addition, Andrea Boltendahl and I generated further 20 PAR-CLIP experiments together. Single PAR-CLIP experiments of Mtr4 and Xrn1 were generated by Saskia Gressel and PARCLIP experiments of Air2, Dcp1, Dcp2, and Edc3 were generated by Dr. Carlo Baejen, who originally started this project.

Table 12: Biological replicates for yeast PAR-CLIP experiments.

\begin{tabular}{|l|l|}
\hline Experiment & Number of replicates in the analysis \\
\hline Air1-TAP & 4 \\
\hline Air2-TAP & 4 \\
\hline Caf40-TAP & 2 \\
\hline Ccr4-TAP & 3 \\
\hline Csl4-TAP & 2 \\
\hline Dcp1-TAP & 2 \\
\hline Dcp2-TAP & 3 \\
\hline Dhh1-TAP & 2 \\
\hline Edc2-TAP & 2 \\
\hline Edc3-TAP & 2 \\
\hline Mtr4-TAP & 3 \\
\hline Nmd4-TAP & 2 \\
\hline Not1-TAP & 2 \\
\hline Pan2-TAP & 3 \\
\hline Pan3-TAP & 3 \\
\hline Pop2-TAP & 2 \\
\hline Rrp4-TAP & 2 \\
\hline Rrp6-TAP & 2 \\
\hline Rrp40-TAP & 2 \\
\hline Rrp44-TAP & 4 \\
\hline Ski2-TAP & 2 \\
\hline Ski3-TAP & 3 \\
\hline Ski7-TAP & 3 \\
\hline Ski8-TAP & 2 \\
\hline Trf4-TAp & 3 \\
\hline Trf5-TAP & 3 \\
\hline Trm140-TAP & 3 \\
\hline Upf1-TAP & 2 \\
\hline Upf2-TAP & 2 \\
\hline Upf3-TAP & 2 \\
\hline Xrn1-TAP & 2 \\
\hline & \\
\hline
\end{tabular}




\subsubsection{Human}

PAR-CLIP of METTL8 was performed with the Flp-In T-Rex cell line expressing METTL8 with a C-terminal 3xFLAG tag. Cells were cultured according to the manufacturer's instruction. Gene expression was induced with $1 \mu \mathrm{g} / \mathrm{ml}$ tetracycline for $24 \mathrm{~h}$. After $23 \mathrm{~h}$, cells were labeled with $500 \mu \mathrm{m} 4-$ thiouridine (Carbosynth) for $1 \mathrm{~h}$ at $37^{\circ} \mathrm{C}$. For UV-crosslinking, medium was removed and cells were washed with PBS. UV-crosslinking was performed with the culture plate on ice and irradiated with $0.9 \mathrm{~J} / \mathrm{cm}^{2}$ at a wavelength of $365 \mathrm{~nm}$. Cells were resuspended in PBS and collected by centrifugation. Cell lysis was performed in 3 volumes (of the cell pellet) lysis buffer on ice for $10 \mathrm{~min}$. For efficient cell lysis, the lysate was passed through a $27 \mathrm{H}$ needle syringe for 10 times. For RNase digest, RNase I (Ambion $100 \mathrm{U} / \mu \mathrm{L}$ ) was added to a final concentration of $200 \mathrm{U}$ and incubated at $37^{\circ} \mathrm{C}$ for $5 \mathrm{~min}$. Immediately after incubation, lysate was incubated on ice for $5 \mathrm{~min}$. RNase digest was finally stopped by adding $20 \mu \mathrm{L}$ SUPERase-In RNase inhibitor $(20 \mathrm{U} / \mu \mathrm{L}$, Thermo Fisher Scientific). Lysate was cleared by centrifugation at $13.000 \mathrm{~g}$ for $10 \mathrm{~min}$ at $4{ }^{\circ} \mathrm{C}$. The supernatant was transferred to $5 \mathrm{~mL}$ Eppendorf tube. Protein concentration was quantified by Bradford assay and input was taken to test IP efficiency. For immunoprecipitation, $100 \mu \mathrm{L}$ Anti-FLAG $®$ M2 Magnetic Beads (Sigma) were washed twice in PBS and lysis buffer per sample. Immunoprecipitation was performed with $25 \mathrm{mg}$ lysate and $100 \mu \mathrm{L}$ beads over night at $4{ }^{\circ} \mathrm{C}$ on a rotating wheel. After IP, beads were collected and washed 3 times with $1 \mathrm{~mL}$ high salt buffer and 1 time with $1 \mathrm{~mL}$ phosphatase buffer. Dephosphorylation of the $5^{\prime}$ RNA end was performed with Antarctic Phosphatase (NEB, M0289S) for $30 \mathrm{~min}$ at $37^{\circ} \mathrm{C}$, shaking at $800 \mathrm{rpm}$. Beads were washed once with Phosphatase wash buffer and twice with PNK buffer. $10 \%$ of IP samples were taken as a control and compared to the input sample by Western Blot. Phosphorylation was performed with 1x T4 PNK reaction Mix with $1 \mathrm{U} / \mu \mathrm{L}$ T4 PNK (Thermo Fisher Scientific) and $0.1 \mu \mathrm{Ci}$ gamma-32P-ATP (Perkin Elmer) for $1 \mathrm{~h}$ at $37^{\circ} \mathrm{C}$, shaking at $800 \mathrm{rpm}$. To ensure all RNAs are fully phosphorylated, ATP (Fermentas) was added to a final concentration of $100 \mathrm{~mm}$ for $5 \mathrm{~min}$ at $37^{\circ} \mathrm{C}, 800 \mathrm{rpm}$. Subsequently, beads were washed 5 times with $1 \mathrm{~mL}$ PNK buffer. $3^{\prime}$ adapter ligation was performed in $40 \mu \mathrm{L}$ of $3^{\prime}$ adapter 
ligation mix (4 $\mu \mathrm{L}$ of $100 \mathrm{~mm} \mathrm{3}$ ' adapter, $20 \mathrm{U} / \mu \mathrm{L}$ T4 RNA Ligase 2 (KQ) (NEB, M0373) over night at $16{ }^{\circ} \mathrm{C}$ and $1 \mathrm{~h}$ at $25^{\circ} \mathrm{C}$, shaking at $600 \mathrm{rpm}$. Beads were then washed 5 times with $1 \mathrm{~mL}$ PNK buffer. For $5^{\prime}$ adapter ligation, beads were resuspended in $40 \mu \mathrm{L} 5^{\prime}$ adapter mix including $4 \mu \mathrm{L} 100 \mathrm{~mm} 5^{\prime}$ adapter and $6 \mathrm{U} / \mu \mathrm{L}$ RNA ligase I (NEB, M0437M) and incubated for $4 \mathrm{~h}$ at $25^{\circ} \mathrm{C}$ and $1 \mathrm{~h}$ at $37^{\circ} \mathrm{C}$, shaking at $600 \mathrm{rpm}$. Beads were washed 5 times with $1 \mathrm{~mL}$ PNK buffer and resuspend in $25 \mu \mathrm{L} 2 \mathrm{X}$ NuPAGE LDS Sample Buffer (Thermo Fisher Scientific). Protein-RNA complexes were released from the beads by boiling for $10 \mathrm{~min}$ at $95^{\circ} \mathrm{C}$. Supernatant was run on a NuPAGE $4-12 \%$ Bis-Tris Polyacrylamide gel (Thermo Fisher Scientific) in MOPS buffer (Thermo Fisher Scientific) in order to separate protein complexes. The phosphor-screen was incubated with the gel and the gel image was visualized using a Typhoon FLA9500 (GE Healthcare Life Sciences). Printout was aligned to the gel and the band corresponding to the protein of interested bound to RNA was excised. Protein bound RNA was eluted from the gel by electro-elution using D-Tube Dialyzer Midi MWCO $35 \mathrm{kDa}$ (EMD Millipore) according to the manufacturer's instructions with $100 \mathrm{~V}$ for $2 \mathrm{~h}$. For proteinase $\mathrm{K}$ digest, the electro-eluate was incubated with $40 \mu \mathrm{L}$ proteinase $\mathrm{K}(800 \mathrm{U} / \mu \mathrm{L}, \mathrm{NEB})$ for $1 \mathrm{~h}$ at $55^{\circ} \mathrm{C}$. RNA was purified by Phenol-Chloroform extraction and ethanol precipitation. Purified RNA was reverse transcribed using Superscript III (Invitrogen, 18080-044). Library generation was performed by PCR amplification using the NEXTflex barcode primer kit (Bio Scientific). 4\% E-Gel Agarose Gel (Invitrogen) was used to size select the cDNA, which was then purified by MinElute gel extraction (Qiagen). cDNA was amplified in a one-step PCR using the Nextera primers and the KAPA Library preparation kit (Roche, Germany). AMPure XP beads (Beckman Coulter) in a ratio of 1:1.8 were used to purify the cDNA library, which was subsequently quantified using TapeStation 2200 (Agilent Technologies) and Qubit (Qiagen). An Illumina machine (HiSeq2500, HiSeq4000 or NextSeq550) was used for sequencing. The experiment was performed in three independent biological experiments. 


\subsection{5 m3C-CLIP}

\subsubsection{S. cerevisiae}

S. cerevisiae wildtype and Trm140 knockout cells were cultured to $\mathrm{OD}_{600} \sim 1.5$ in SCM minimal medium (Formedium) supplemented with $10 \mathrm{mg} / \mathrm{L}$ uracil, $100 \mathrm{~mm}$ 4-thiouracil (4tU), and $2 \%$ glucose. Cells were lysed by bead beating and RNA was recovered by acidic phenol/chloroform extraction.

$500 \mu \mathrm{g}$ of RNA were immunoprecipitated with $30 \mu \mathrm{L}$ anti-m3C antibody (Active Motif) for $2 \mathrm{~h}$ at $4{ }^{\circ} \mathrm{C}$. Antibody-RNA complexes were UV-irradiated with an energy dose of $0.45 \mathrm{~J} / \mathrm{cm}^{2}$ at a wavelength of $365 \mathrm{~nm}$. Antibody-RNA complexes were captured using protein $\mathrm{G}$ magnetic Dynabeads (Invitrogen) for $2 \mathrm{~h}$ at $4{ }^{\circ} \mathrm{C}$. Crosslinked RNA was partially digested with RNase $\mathrm{T} 1$ and used for cDNA library preparation. RNA was recovered by Proteinase $K$ digestion for $2 \mathrm{~h}$ at $55^{\circ} \mathrm{C}$ and subsequent acidic phenol/chloroform extraction and ethanol precipitation. Reverse transcription was performed using SuperScript III RTase (Invitrogen). PCR amplification was done using the NEXTflex barcode primer kit (Bio Scientific). Generated cDNA was purified, size-selected, and quantified using TapeStation (Agilent Technologies). Samples were sequenced on an Illumina machine (HiSeq2500, HiSeq4000 or NextSeq550).

\subsubsection{Human}

m3C-CLIP of human cells was performed with the FIp-In T-Rex cell line expressing METTL8 with a C-terminal 3xFLAG tag. Cells were cultured as described. Gene expression was induced with $1 \mu \mathrm{g} / \mathrm{mL}$ tetracycline for $24 \mathrm{~h}$. After $23 \mathrm{~h}$, cells were labeled with $500 \mu \mathrm{M}$ 4-thiouridine (Carbosynth) for $1 \mathrm{~h}$ at $37^{\circ} \mathrm{C}$. Cells were washed in PBS, collected by centrifugation, and resuspended in QIAzol (Qiagen) according to the manufacturer's instruction. RNA was purified using ethanol precipitation and a fraction of it was demethylated with AlkB (see section 3.2.8) as control. The subsequent protocol starting with RNA immunoprecipitation was performed as described in yeast in section 3.2.5.1. 


\subsection{6 $4 \mathrm{tU}-\mathrm{seq}$ in S. cerevisiae}

WT and Trm140 KO strains were grown in yeast extract peptone dextrose (YPD) overnight at $30^{\circ} \mathrm{C}$. Cultures were diluted to $\mathrm{OD}_{600} \sim 0.1$ and cultured to $\mathrm{OD}_{600} \sim 0.6$. $4 \mathrm{tU}$ labeling was subsequently performed as described (Sun et al., 2012). Labeled RNA was purified from extracted total RNA. The Ovation Universal RNA-Seq System Library Kit (NuGen) was used according to the manufacturer's instructions with an input of $100 \mathrm{ng}$ of labeled RNA. The Fragment Analyzer and Qubit (Invitrogen) were used to check the quality and quantity of the libraries. Libraries were pooled and sequenced on an Illumina Sequencer (NextSeq550).

\subsubsection{Protein purification of AlkB and Trm140}

Geneblocks coding for the respective protein were purchased from IDT and cloned into His6-TEV-tag containing LIC expression vectors in XL1 Blue cells supplemented with Kanamycin. Positive plasmids were transformed into BL21DE3RIL cells. Cells were grown in LB medium at $37^{\circ} \mathrm{C}$ until $\mathrm{OD}_{600} \sim 0.6$ and protein expression was induced by the addition of IPTG with final concentration of $1 \mathrm{~mm}$ for $3 \mathrm{~h}$. All purification steps were performed at $4{ }^{\circ} \mathrm{C}$. Cells were resuspended and lysed in buffer A using a sonicator for $5 \mathrm{~min}$ at $60 \%$ output. The lysate was applied to a HisTrap column and washed with buffer B. Protein was eluted with buffer C. Fractions containing His6-TEVprotein were collected, TEV was added and dialyzed against buffer D. Sample was applied to a HisTrap column and flow through was collected. Gel filtration was performed on a HiLoad 16/600 Superdex 75 pg using buffer E. For Trm140 an additional Q column after His6-tag removal was applied and protein was eluted with buffer $B$. 


\subsubsection{RNA demethylation using AlkB}

RNA demethylation was performed as described (D'Silva et al., 2011). Briefly, purified RNA (50-1000 $\mu \mathrm{g})$ isolated from yeast was incubated with purified AlkB from E. coli (see Methods section 2.2.7) in buffer containing $50 \mathrm{~mm}$ Hepes $\mathrm{KOH}\left(\mathrm{pH}\right.$ 8), $75 \mathrm{~mm} \mathrm{Fe}\left(\mathrm{NH}_{4}\right)_{2}\left(\mathrm{SO}_{4}\right)_{2} \cdot 6 \mathrm{H}_{2} \mathrm{O}, 1 \mathrm{~mm}$ a-ketoglutarate, $2 \mathrm{~mm}$ $\mathrm{Na}$-ascorbate, and $50 \mathrm{mg} / \mathrm{mL} \mathrm{BSA}$ for $1 \mathrm{~h}$ at $37^{\circ} \mathrm{C}$. The reaction was stopped using a final concentration of $11 \mathrm{mM}$ EDTA (Trewick et al., 2002), followed by phenol/chloroform extraction and ethanol precipitation to recover the RNA.

\subsubsection{RNA re-methylation using Trm140 and SAM}

Re-methylation assay was performed as described (D'Silva et al., 2011). Briefly, demethylated or Trm140 KO RNA was treated with $50 \mathrm{~mm}$ Tris- $\mathrm{HCl} \mathrm{pH}$ 8.0, $1 \mathrm{~mm}$ DTT, $0.1 \mathrm{~mm}$ EDTA, $1 \mathrm{~mm}$ spermidine, $0.5 \mathrm{~mm}$ SAM, and purified Trm 140 for $1 \mathrm{~h}$ at $30{ }^{\circ} \mathrm{C}$, followed by phenol extraction and ethanol precipitation for recovery of the RNA.

\subsubsection{Reverse transcription assay and denaturing PAGE}

S. cerevisiae wildtype and Trm140 knockout cells were grown to $\mathrm{OD}_{600} \sim 1.5$ in YPD. Cells were lysed by bead beating and RNA was recovered by acidic phenol/chloroform extraction. 20 to $80 \mu \mathrm{g}$ of RNA were used for reverse transcription for $1 \mathrm{~h}$ at $44^{\circ} \mathrm{C}$ and $1 \mathrm{~h}$ at $55^{\circ} \mathrm{C}$ using SuperScript III RTase (Invitrogen). Products were separated on a 15\% 8 M Urea gel at $300 \mathrm{~V}$. Products were visualized on a Typhoon FLA 8500 using 5' FAM labeled RT primers for tRNA Serine (5'- CGACACCAGCAGGATTTGAA-3') and (Arginine (5'-CGTTCCGTACGGGACT-3'). 


\subsection{Data analysis}

\subsubsection{PAR-CLIP data pre-processing}

This analysis was performed by Salma Sohrabi-Jahromi. "Reads from PAR-CLIP experiments with replicates were merged after making sure that all samples showed high Spearman correlation values comparing binding occupancies of replicates on different genes (Figure 7B). Mapping and statistical evaluation of PAR-CLIP experiments was performed using our inhouse software mockinbird (Roth and Torkler, 2018). In summary, the UMI is removed from the $5^{\prime}$ end with UMI-tools (T. Smith et al., 2017), and the $3^{\prime}$ adapter is trimmed with Skewer (Jiang et al., 2014). Reads with traces of the $5^{\prime}$ adapter are discarded. The preprocessed reads are then mapped to the $\mathrm{S}$. cerevisiae genome (sacCer3, version 64.2.1). After mapping PCR duplicates are removed with UMI-tools.

We used two alternative approaches for mapping reads using Bowtie (Langmead et al., 2009): For all analyses except the 'transcript class enrichment analysis' in Figure 8, reads are uniquely mapped with up to one mismatch. We discard alignments shorter than $20 \mathrm{nt}$. This stringent mapping ensures that our high confidence PAR-CLIP cross-link sites are originating from correctly mapped reads on the reference genome. For Figure 8, unique mapping would cause the loss of most reads that fall into rRNAs and tRNAs because of duplicated rRNA genes and tRNA isodecoders. For Figure 8, we therefore allowed Bowtie multi-mapping in two regions with -best, -starra options and discarded reads shorter than $30 \mathrm{nt}$.

$T \rightarrow C$ transitions directly at the edge of the reads or with a Phred quality score lower than 20 are not considered as signature of protein binding as they suffer from higher technical noise. To obtain high confidence cross-link sites, we set a stringent cutoff of 0.005 for the p-value of cross-link sites and require a minimum coverage of 2 per site. Moreover, if we see the same transition in at least $75 \%$ of reads in the input library control (SRA: SRX532381) (Baejen et al., 2014), we annotate it as a single nucleotide polymorphism of our lab strain with respect to the genomic reference and remove such sites from our analysis. Finally, the occupancy of a factor on a verified cross-link site is defined as the 
number of transitions obtained from our PAR-CLIP experiments divided by the concentration of RNAs covering the cross-linked site according to the input library control. This control coverage is measured under comparable conditions to PAR-CLIP experiments (Baejen et al., 2014). Occupancy values are capped at the $95^{\text {th }}$ percentile. Subsequent analyses were performed using in-house python scripts. Mockinbird configuration files as well as the analysis scripts can be found at https://github.com/soedinglab/Degradation_scripts."

\subsubsection{Transcript class enrichment}

The following analysis was performed by Salma Sohrabi-Jahromi. "We analyzed the distribution of reads from high-confidence cross-link sites over the genome (Figure $8 \mathrm{~A}$ ). We presented the sum of reads from $5^{\prime}$ and $3^{\prime}$ UTRs, coding sequences, and introns as the value for mRNAs. Reads that fall within genomic regions not annotated as categories analyzed here are shown with grey. These annotated transcript classes have comparable U-content, making the comparison between fractions of cross-link sites in each category possible (Appendix Figure 28).

For each factor studied here, we defined enrichment scores that represent their preferences for binding to various transcript classes $c$, in comparison to all other factors. We use annotations for rRNA, tRNA, snoRNA, snRNA, coding sequences (CDS), from S. cerevisiae genome sacCer3, version 64.2.1. Untranslated regions around coding boundaries ( $5^{\prime}$ and $3^{\prime}$ UTRs) were annotated based on TIF-seq experiment (Pelechano et al., 2013). We selected the most strongly expressed isoform for each gene. We then assigned boundaries to $3^{\prime}$ and $5^{\prime}$ UTRs based on annotated CDS of the same gene. We furthermore used annotations for stable, unannotated transcripts (SUTs), cryptic unstable transcripts (CUTs), and Nrd1- unterminated transcripts (NUTs) (Neil et al., 2009; Pelechano et al., 2013; Schulz et al., 2013). We removed overlapping annotations with the following priority list: rRNA, tRNA, snRNA, snoRNA, intron, CDS, UTR, SUT, CUT, NUT. For each factor, we counted the number of high-confidence reads falling in each transcript class. We then used the $\log _{2}$-transformed matrix and normalized it in the following way for both rows 
and columns to get log enrichment values that sum to zero in both rows and columns. The row- and sum-normalized enrichment score is defined as follows, where $X_{f, c}$ is the number of high-confidence reads for factor $f$ that fall into transcript class $c$, and $X_{f, c}^{\prime}=\log _{2} X_{f, c}$ (Figure 8B):

$$
\tilde{X_{f, c}^{\prime}}=X_{f, c}^{\prime}-\frac{X_{f, \circ}^{\prime} X_{\circ, c}^{\prime}}{X_{\circ, \circ}^{\prime}}
$$

We defined the row and sum averages of $X_{f, c}$,

$$
\begin{gathered}
X_{f, \circ}^{\prime}=\frac{1}{C} \sum_{c=1}^{C} X_{f, c}^{\prime}, \\
X_{\circ, c}^{\prime}=\frac{1}{F} \sum_{f=1}^{F} X_{f, c}^{\prime}, \\
X_{\circ, \circ}^{\prime}=\frac{1}{F C} \sum_{f=1}^{F} \sum_{c=1}^{C} X_{f, c}^{\prime}
\end{gathered}
$$

$F$ is the number of factors and $C$ is the number of transcript classes (Figure 8B). The normalization can be interpreted as subtracting from the log enrichment matrix $X$ ' the first singular component of its singular-value decomposition."

\subsubsection{Metagene analysis}

This analysis was performed by Salma Sohrabi-Jahromi. "We used the most abundant TIF-annotated isoform for mRNAs (Pelechano et al., 2013) as a reference. Transcripts longer than 1500 bases are chosen and aligned at their TSS or pA sites. The average occupancy per nucleotide is then calculated based on high-confidence cross-link sites of each PAR-CLIP experiment. The profiles are smoothed by a moving average in a $41 \mathrm{nt}$ window and the $95 \%$ 
confidence interval is estimated by 1500 bootstrap sampling iterations over the transcripts. To further denoise the profiles, the cross-link sites falling in snRNAs, rRNAs, and tRNAs are removed. Furthermore, to avoid ambiguous results, we made sure that the profile comes solely from the central gene. To do so, we performed the metagene analysis around the TSS on the sense strand on TIF-annotated mRNAs that have no other mRNA up to $700 \mathrm{bp}$ upstream of their TSS (3193 transcripts in total). Analogously, for sense-strand pA site profiles we used mRNAs that have no nearby genes downstream of their pA site up to 700 bases on the same strand (3193 transcripts in total). For the antisense strand profiles, we applied the same criteria on the opposite strand which left us with 3076 and 3193 transcripts filtered around TSS and pA sites respectively. This ensures that the observed antisense binding does not originate from neighboring or overlapping transcripts on the antisense strand. In both cases we looked at the average occupancy in a window of [ $\pm 700 \mathrm{nt}]$ around TSS and around PA sites. Occupancies were normalized to the maximum value, which is the background binding level for antisense profiles with no significant cross-linking to the antisense strand (Figure 9 and Figure 10). The same procedure was followed to plot metagene occupancies centered around protein-coding regions and snoRNAs from S. cerevisiae genome sacCer3, version 64.2.1 (Appendix Figure 29 and Figure 39)."

\subsubsection{Co-occupancy}

Salma Sohrabi-Jahromi performed the following analysis. "Cooccupancy measures the tendency of two factors to bind to the same transcripts. Occupancy of a factor on a transcript is defined as the sum of occupancies for all high-confidence cross-link sites falling within this transcript. Co-occupancy of two factors is defined as the Pearson correlation over all transcripts between the occupancies of these factors (Figure 11A). We used these correlation values between all pairs of RNA processing factors to assign distances to each pair and used tSNE (Van Der Maaten and Hinton, 2008) to visualize the two-dimensional nonlinear embedding of co-occupancies for all RNA-binding proteins in our dataset (Figure 11C).“ 


\subsubsection{Co-localization}

This analysis was performed by Salma Sohrabi-Jahromi. "Colocalization measures how likely two factors are to bind near each other in the transcriptome. More precisely, we first calculate the occupancy of a factor $f$ $\in\{1, \ldots, F\}$ around the cross-link sites of another factor $f^{\prime}([-40 \mathrm{nt},+40 \mathrm{nt}]$ excluding the centered $\mathrm{T}$ ). We then normalize according to the total occupancy values,

$$
\begin{gathered}
z_{f f^{\prime}}=\sum_{i=1}^{n_{f^{\prime}}}\left(\sum_{j=-40}^{-1} O c c_{f f^{\prime}, i, j}+\sum_{j=1}^{40} O c c_{f f^{\prime}, i, j}\right) \\
\text { co-localization }\left(f, f^{\prime}\right)=\frac{z_{f f^{\prime}}}{\sum_{f} z_{f f^{\prime}} \sum_{f^{\prime}} z_{f f^{\prime}}}
\end{gathered}
$$

Where, $\mathrm{n}_{f}$ is the number of cross-link sites for factor $f$, and Occffi, is the occupancy of $f$ at position $j$ around the $i^{\text {th }}$ cross-link site from factor $f^{\prime}\left(\mathrm{Occff}^{\prime}, i, j=\right.$ 0 if no verified cross-link sites exist). To improve signal-to-noise, we compute from the resulting matrix of co-localizations between all RNA-processing factors $\mathrm{C}_{\mathrm{f}, \mathrm{f}^{\prime}}$, the matrix of Pearson correlations between the rows of $\mathrm{C}_{\mathrm{f}, \mathrm{f}^{\prime}}$, (Figure 11B, Figure 21, Appendix Figure 41).“

\subsubsection{Codon-enrichment analysis}

The following analysis was performed by Salma Sohrabi-Jahromi. "To search for possible links between translation efficiency and RNA degradation, we checked if some degradation factors preferentially bind to translationally efficient/non-efficient transcripts. To do so we adapted the proposed normalized translation efficiency scale (Pechmann and Frydman, 2013). The authors generate a normalized optimality score for codons that incorporates the competition between supply and demand of tRNAs. The coding region for each transcript was extracted according to ORFs annotated by SGD. The codon 
optimality score was averaged over the whole reading frame (Figure 12A, more detailed explanation in the next section).

We then checked whether mRNAs that bind to each factor are enriched or depleted in some codons compared to all mRNAs. To achieve this, we defined the following score for codon enrichment that represents deviations from average frequencies in all mRNAs,

$$
\text { codon enrichment }=\frac{\sum_{t=1}^{T}\left(\frac{o c c(t)}{\sum_{t^{\prime}=1}^{T} o c c\left(t^{\prime}\right)} \times F_{c, t}\right)}{\frac{1}{T} \sum_{t=1}^{T} F_{c, t}}
$$

Here $T$ is the number of mRNA transcripts, $F_{c, t}$ is the fraction of the codon $c$ in transcript $t$, and $\operatorname{coc}(t)$ is the total occupancy of the factor on transcript $t .90 \%$ confidence intervals were generated by bootstrapping: we sampled with replacement 1000 times the same number of mRNAs from the total set as in total, and for each set we recalculated the codon enrichment score. We colored the bars based on the previously ranked optimality of codons (Pechmann and Frydman, 2013) (Figure 12B, Appendix Figure 31-Figure 37)."

\subsubsection{Relating occupancies to various transcript features}

This analysis was performed by Salma Sohrabi-Jahromi. "We analyzed the correlation of the occupancy of all factors with transcript length, codon enrichment of the transcript, expression level, transcript stability, and polyA tail length. For expression, we used an RNA-seq experiment of wild-type yeast (SRA: SRX532381) (Baejen et al., 2017) and mapped the reads to mRNAs. We present the average number of reads per base as an estimate for gene expression. For half-life calculations, we used published yeast 4tU-seq (GEO: GSM2199309) and RNA-seq experiments (SRA: SRX532381) (Baejen et al., 2017). Transcript half-life is estimated with an optimized method that will be published elsewhere (Hofmann et al., unpublished). 
Since there are only few transcripts with very low or very high half-life, codon optimality, and expression (Appendix Figure 30), we performed the analysis on a subset of mRNAs where the transcript property lies between the $5 \%$ and $95 \%$ quantiles. We then compared the total occupancy of degradation factors on each mRNA relative to such transcript features (Figure 12A, Figure 13B, and Appendix Figure 31-Figure 37). We show 95\% confidence intervals generated by bootstrapping mRNAs in grey shade.

We checked whether such correlations originate from the feature of interest or merely shows up due to correlations between this feature and others (Appendix Figure 30). We used a multivariate linear regression to model total occupancy as a linear function of these four features:

$$
\text { occupancy }(t) \sim \text { length }+ \text { optimality }+ \text { expression }+ \text { halflife }
$$

In cases where the correlation is a direct effect from our feature of interest, we expect to lose significantly on our prediction when this variable is taken out of the equation. Therefore, we use p-values representing the importance of each feature in this linear regression as a score representing the significance of its contribution in explaining the final occupancies. Occupancy correlated strongly with transcript length, which dominated as explanatory variable in this regression, trivially because most factors bind along the entire transcript. To eliminate this trivial dependency, we used occupancy per nucleotide, denoted occupancy', as the target variable in our regression (Figure 12C)."

\subsubsection{Motif enrichment analysis}

Salma Sohrabi-Jahromi performed the following analysis. "To find sequence preferences for binding events of degradation factors, we counted 4mers in a window of $[ \pm 5 \mathrm{nt}]$ intervals around high-confidence cross-link sites of PAR-CLIP experiments. Based on this count table, the enrichment score for each 4-mer was calculated using the following formula, 


$$
4 \text {-mer enrichment }=\frac{n_{4-\mathrm{mer}, i}+1}{N \times \prod_{j=1}^{4} P_{4-\operatorname{mer}[j]}}
$$

Here $N$ is the number of cross-link sites below the cut-off $p$-value (we used a maximum of 5000 cross link sites), $n_{4 m e r, i}$ is the number of observed 4-mers at position $i$ in the set of binding sequences aligned at their cross-link site $i=0$, 4-mer[] is the base at the $j$ th position of the 4-mer, and $P_{b}$ is the probability of observing base $b$. We used the probabilities: $P_{A}=P_{T}=0.31$ and $P_{C}=P_{G}=0.19$ based on frequencies in yeast genome and corrected for the T bias at the crosslink site (Figure 13C)."

\subsubsection{Trm140 PAR-CLIP and m3C-CLIP analysis}

This analysis was performed by Gabriel J. Villamil. Sequencing reads of independent biological replicates from Trm140 PAR-CLIP and m3C-CLIP sequencing experiments were merged. Single-end $50 \mathrm{nt}$ and $75 \mathrm{nt}$ reads were mapped to the R64-2-1 S. cerevisiae reference genome (Engel et al., 2013) using STAR 2.5.3a (Dobin and Gingeras, 2015) with a maximum of two mismatches allowed per alignment. In cases of multi-mapped reads, the single best alignment was taken. Trm140 and anti-m3C antibody binding was determined from chemical cross-links indicated by $T \rightarrow C$ transitions called using a statistical model as described (Baejen et al., 2017, 2014) with a maximum pvalue threshold of 0.005 and a minimum coverage of 2 . The Phred score of sequencing quality at the transition base was set to 20. RNA-seq data obtained under similar conditions to PAR-CLIP experiments was used to remove potential transition miscalls caused by SNPs. Briefly, transitions in PAR-CLIP reads that are also observed in at least $75 \%$ of RNA-seq reads covering the same base were considered as SNPs and removed from the analysis. Accumulation of binding sites are depicted metagene-wide aligned at the transcription start site (TSS) and polyadenylation site (pA) of designated loci. 
3.3.10 Calling of $\mathrm{m} 3 \mathrm{C}$ modification sites

Sites of $\mathrm{m} 3 \mathrm{C}$ modification were determined from $\mathrm{C} \rightarrow \mathrm{T}$ transitions in PAR-CLIP reads. $C \rightarrow T$ transitions were called with a transition rate of a least $4 \%$, a minimum coverage of 40 , and a minimum sequencing quality of 20 at the transition base resulting in the high confidence list of $\mathrm{m} 3 \mathrm{C}$ sites shown in Appendix Table 14.

\subsubsection{Calculation of ribosome P site occupancy}

This analysis was performed by Gabriel J. Villamil. We calculated the ribosome $\mathrm{P}$ site occupancy based on the data from WT and Trm140 KO ribosome profiling experiments (Chou et al., 2017). Briefly, global codon occupancy analysis was calculated (Nedialkova and Leidel, 2015) with minor modifications. Examination of the cumulative distribution of $28-31 \mathrm{nt}$ reads aligned at the start codons using Plastid (online package) revealed the $\mathrm{P}$ site offset. The respective offset was applied to reads of each size and off frame sequencing reads were discarded. We removed the first 15 and last 5 codons of each transcript from the reference. The quotient of frequency of each codon in ribosomal $\mathrm{A}, \mathrm{P}$ and $\mathrm{E}$ site to the average frequency of the same codon in the three downstream codons from the A site was used for normalization.

3.3.12 Calculation of translational efficiency on m3C containing codons

Translation efficiency (TE) was calculated by using the averaged sequencing reads of ribosome protected fragments from two independent biological replicates of WT and Trm140 KO ribosome profiling experiments at $\mathrm{m} 3 \mathrm{C}$ containing codons and divided by the number of RNA-seq reads from two independent biological replicates of WT and Trm140 KO cells at the same codon (Chou et al., 2017). The following m3C containing transcripts were selected based on the mutational signature in the Trm140 PAR-CLIP experiments: YHR099W (at position chrVIII:303,572), YDR341C (at position 
chrlV:1,153,577), YPR148C (at position chrXVl:827,581), YEL060C (at position chrV:41,712), YML058W (at position chrVIII:159,468), and YOR361C (at position chrXV:1,017,134). The change in TE was plotted using Microsoft $®$ Excel (Microsoft Office 2017) for each codon in Figure 20B.

\subsubsection{4tU-seq data pre-processing and normalization}

Gabriel J. Villamil performed the following analysis. Paired-end $50 \mathrm{nt}$ sequencing reads were mapped to the R64-2-1 S. cerevisiae reference genome assembly (Engel et al., 2013) using STAR 2.5.2b (Dobin and Gingeras, 2015) with a maximum of two mismatches allowed per alignment and maximum intron length set to 500,000. In cases of multi-mapped reads, the single best alignment was taken. Sequences of labelled and unlabelled RNA spike-ins were included as additional chromosomes in the reference sequence to map reads from spike-ins. SAMtools 1.3.1 ( $\mathrm{Li}$ et al., 2009) was used to remove alignments with MAPQ scores lower than 7 and to sort and index BAM files. Piled-up reads on transcription units were counted using HTSeq 0.6.1p1 (Anders et al., 2015). Antisense bias correction was performed as described (Gressel et al., 2017). Briefly, counts of reads mapping antisense to spike-in sequences were used to measure the extent of antisense bias and correct counts for all reads.

Real read counts in labelled RNA samples $L$ or in total cellular RNA samples $T$ for transcription unit $i$ in sample $j$ were calculated as:

$$
\begin{aligned}
& L_{i j}=\frac{k_{i j}^{L}}{l_{i} \sigma_{j}^{L}} \\
& T_{i j}=\frac{k_{i j}^{T}}{l_{i} \sigma_{j}^{T}}
\end{aligned}
$$

Here, $k_{i j}$ is the raw read count of a transcription unit in a labelled or total sample, $l_{i}$ is the length of the transcription unit, and $\sigma_{j}$ is a scaling factor that accounts for variations in sequencing depth determined from spike-in counts. 
3.3.14 Estimation of RNA synthesis and degradation rates

The following analysis was performed by Gabriel J. Villamil. Estimates of gene-specific amounts of labelled RNA $\alpha_{i j}$ and unlabelled RNA $\beta_{i j}$ in a tuple of labelled and total sample were modelled as:

$$
\begin{gathered}
\alpha_{i j}=\frac{L_{i j}-\epsilon_{j}^{L} T_{i j}}{1-\epsilon_{j}^{L}} \\
\beta_{i j}=\frac{T_{i j}-L_{i j}}{1-\epsilon_{j}^{L}}
\end{gathered}
$$

Here, $\epsilon_{j}$ is a cross-contamination rate that models the proportion of unlabelled reads purified in the labelled sample.

We extend the statistical model to estimate gene-specific synthesis rates $\mu_{i j}$ and degradation rates $\lambda_{i j}$, assuming first-order kinetics using the following equations:

$$
\begin{gathered}
\alpha_{i j}=\frac{\mu_{i j}}{\gamma_{j}+\lambda_{i j}} \cdot\left(1-e^{-t\left(\gamma_{j}+\lambda_{i j}\right)}\right) \\
\alpha_{i j}+\beta_{i j}=\frac{\mu_{i j}}{\gamma_{j}+\lambda_{i j}}
\end{gathered}
$$

Here, $t$ is the labelling duration of 6 minutes, and therefore:

$$
\begin{aligned}
& \lambda_{i j}(t)=-\gamma_{j}-\frac{1}{\mathrm{t}} \cdot \log \left(\frac{\beta_{i j}}{\alpha_{i j}+\beta_{i j}}\right) \\
& \mu_{i j}(t)=\left(\alpha_{i j}+\beta_{i j}\right) \cdot\left(\gamma_{j}+\lambda_{i j}(t)\right)
\end{aligned}
$$

$\gamma_{j}$ is the dilution rate of RNA due to cell growth, it is calculated as:

$$
\gamma_{j}=\frac{\log (2)}{C C L_{j}}
$$

where $C C L_{j}$ is the length of one cell cycle in minutes. 
In order to maximize the number of successfully calculated decay rate values, labelled read counts $L_{i j}$ were optimized across all samples with a common scaling factor that minimizes the occurrence of logarithms of negative values and negative decay rate values, both of which were excluded from further analysis (Figure 23). The inclusion of this factor does not affect the relative observations made in this study. 


\section{Transcriptome maps of general eukaryotic RNA degradation factors}

\subsection{Results}

"In order to get a better understanding of RNA processing and degradation in a eukaryotic cell, we measured transcriptome-wide binding locations of 30 RNA degradation factors involved in mRNA deadenylation, decapping, exosome-mediated degradation, and in RNA surveillance pathways including nuclear RNA surveillance and cytoplasmic nonsense-mediated decay (NMD) (Table 12). We performed PAR-CLIP in S. cerevisiae using our published protocol (Battaglia et al., 2017), with minor modifications (Methods). The high reproducibility of these PAR-CLIP experiments is revealed by a comparison of two independent biological replicates that we collected for all 30 degradation factors (Figure 7B), with Spearman correlations between 0.87 and 1.00 (mean: 0.94). We typically obtained tens of thousands of verified factorRNA cross-link sites with p-values $\leq 0.005$ (Figure 7A). These transcriptome maps represent an extensive, high-confidence dataset of in vivo RNA-binding sites for factors involved in RNA degradation." 
A

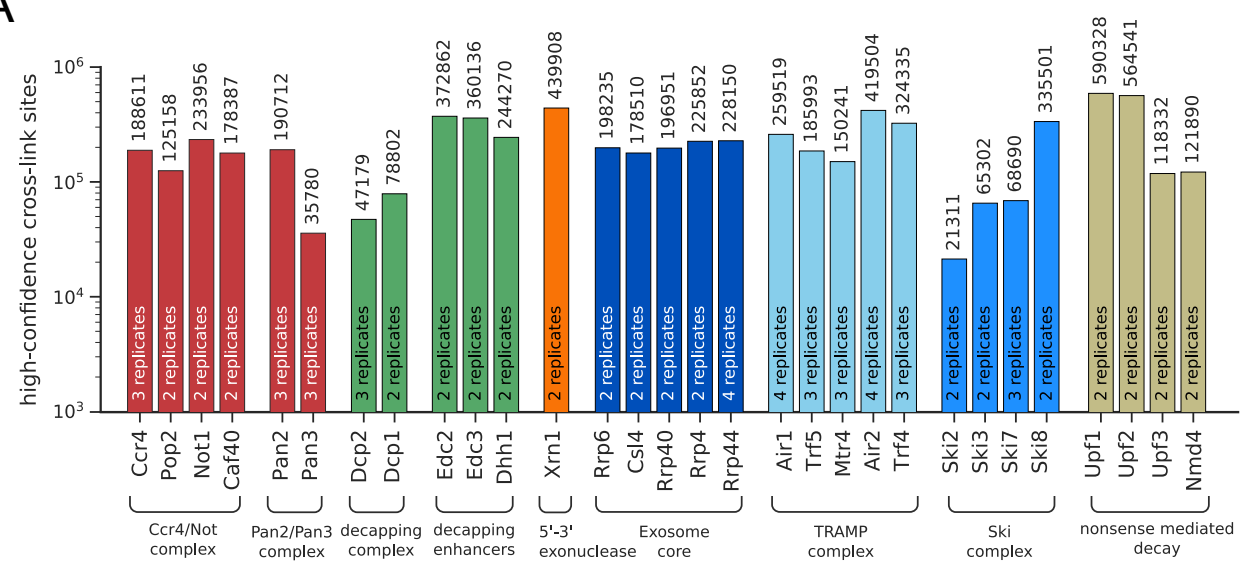

B
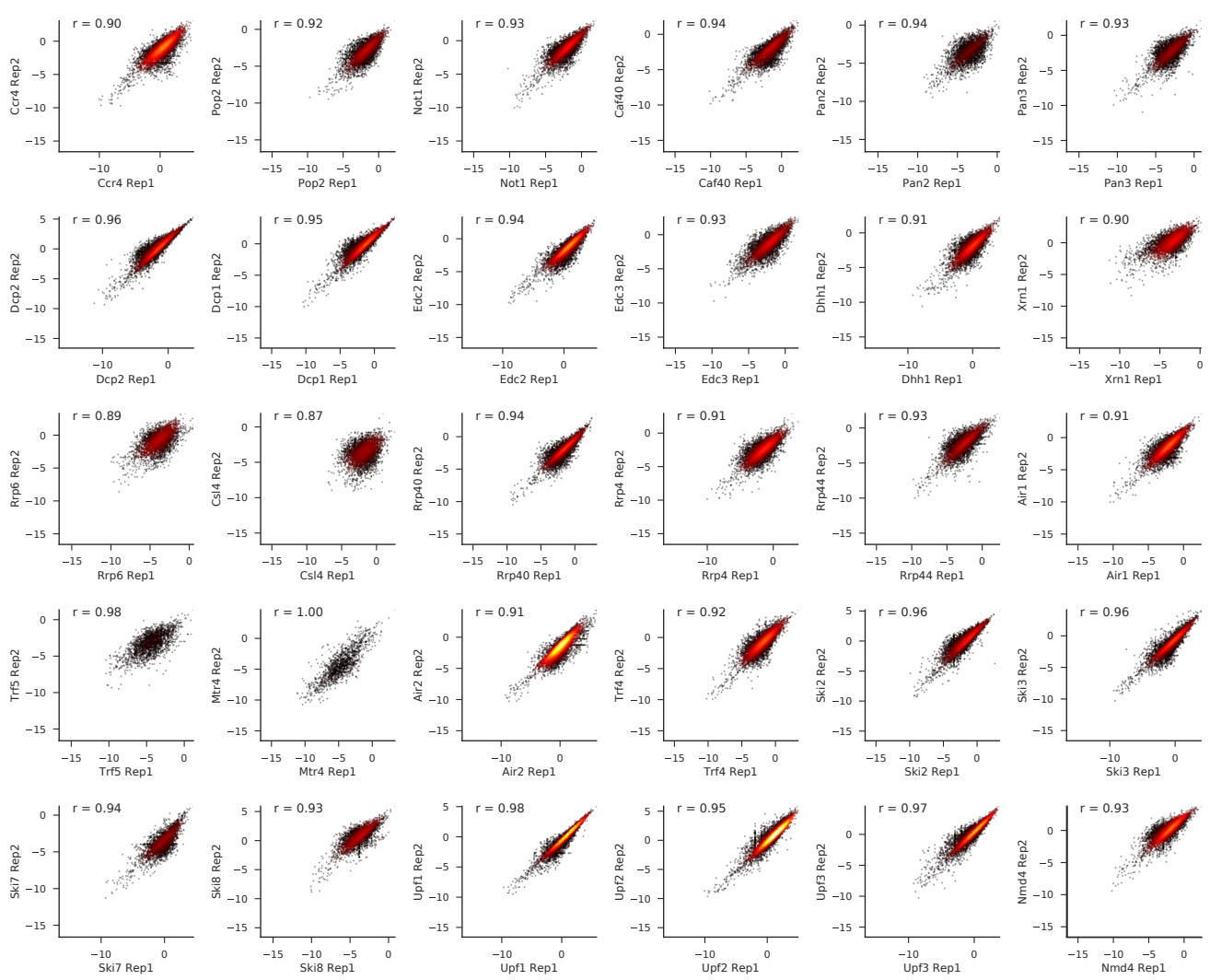

Figure 7: Number of PAR-CLIP cross-link sites and replicate correlation.

A) The number of high-confidence PAR-CLIP cross-link sites for each factor after merging of sequencing files of independent biological replicates is shown as a bar plot. The factors are sorted according to the complex they are residing in and colored in red (deadenylation), green (decapping), orange (exonuclease), dark blue (exosome), middle blue (TRAMP), light blue (Ski), and ocherous (NMD). B) Total transcript occupancy of all 30 factors in replicate experiments are plotted in log2 space and Spearman correlation values are shown for each pair. Perfectly correlating pairs are located on the diagonal of the plotting area. 


\subsubsection{Degradation factors exhibit transcript class specificity}

"We first compared degradation factor binding over different RNA classes. These included protein-coding messenger RNA (mRNA), where we distinguished the $5^{\prime}$ untranslated region ( $5^{\prime}$ UTR), the coding sequence (CDS), introns, and the $3^{\prime}$ untranslated region ( $3^{\prime}$ UTR). We also included several classes of ncRNAs: ribosomal (r), transfer ( $\mathrm{t}$ ), small nucleolar (sno), and small nuclear (sn) RNAs, as well as stable unannotated transcripts (SUTs), cryptic unstable transcripts (CUTs), and Nrd1- unterminated transcripts (NUTs) (Neil et al., 2009; Pelechano et al., 2013; Schulz et al., 2013) (Figure 8).

A first analysis revealed that most PAR-CLIP sequencing reads fall into the mRNA transcript class, although many of the factors also show a considerable number of sequencing reads in ncRNAs, in particular rRNAs (Figure 8A). To obtain a more quantitative comparison, we defined log enrichment scores that reflect the preferences of factors in binding to a specific transcript class in comparison to other factors and classes. To correct for the different sizes of classes and different numbers of measured factor binding sites, we normalized the log enrichment scores by subtracting class- and factorspecific offsets, such that the mean for each class and each factor vanishes (Figure 8B, Methods). This analysis highlights differences between degradation factors with respect to binding to various transcript classes, as will be discussed in detail below."

\subsubsection{RNA end- processing complexes differ in their targets}

"The catalytic subunit Pop2 and the core subunits Not1 and Caf40 of the deadenylase complex $\mathrm{Ccr} 4 / \mathrm{Not}$ have similar binding preferences for the $5^{\circ}$ UTR, the CDS and $3^{\prime}$ UTR of mRNAs, for rRNAs, tRNAs, snoRNAs, and snRNAs (Figure 8B, highlighted in red). Compared to other deadenylation factors of the Ccr4/Not complex, the catalytically active subunit Ccr4 has different binding preferences, and is strongly enriched at mRNA introns. The second deadenylation complex, Pan2/Pan3, shows a similar binding preference as the Ccr4/Not complex (except for the Ccr4 subunit), consistent 
with its dominant role in yeast mRNA deadenylation (Boeck et al., 1996). Pan3 shows a strong binding preference for rRNAs and tRNAs.

For all decapping-related factors we observed similar binding preferences among each other (Figure 8B, highlighted in green). They show the strongest enrichment at SUTs and at mRNAs compared to the other transcript classes. Decapping factors bind preferentially to CDS and 3' UTR, as well as to SUTs. This is consistent with previous findings that SUTs are degraded via Dcp2-dependent pathways in the cytoplasm (Marquardt et al., 2011; Smith et al., 2014; Thompson and Parker, 2007). Dcp2, which harbors the hydrolase activity that removes the $5^{\prime}$ cap, and the decapping activator Edc3, additionally bind to NUTs. The $5^{\prime}$ exonuclease Xrn1 shows a similar binding preference as the decapping factors (Figure 8B, highlighted in orange). Taken together, complexes and enzymes that are known to target mRNA ends for $3^{\prime}$ deadenylation and $5^{\prime}$ decapping and degradation show remarkably distinct binding specificities to different transcript classes."

\subsubsection{The exosome and surveillance factors}

"For the exosome we also observed binding to different RNA classes (Figure 8B, highlighted in royal blue). The core exosome subunits CsI4 and Rrp40 showed similar cross-linking to rRNAs, tRNAs, snoRNAs, and snRNAs. The catalytic exosome subunit Rrp44 and the core subunit Rrp4 binds to introns of mRNAs, but preferentially to the short-lived, nuclear CUTs and NUTs. Rrp6, a subunit that is exclusively present in the nuclear exosome complex, shows binding to rRNAs, snoRNAs, snRNAs, CUTs and NUTs. This is consistent with the suggestion that the factor is needed for nuclear processing of such noncoding transcripts and degradation of short-lived nuclear transcripts (Heo et al., 2013; Vasiljeva and Buratowski, 2006). This complex distribution of cross-links for different exosome subunits to different RNA classes reflects the distinct functions of the exosome in nuclear RNA surveillance, processing of stable ncRNAs, and cytoplasmic mRNA degradation (Zinder and Lima, 2017).

The two TRAMP complexes TRAMP4 and TRAMP5 show clearly distinct cross-linking patterns (Figure 8B, highlighted in light blue). TRAMP4 subunits 
(Mtr4, Air2, Trf4) are enriched in introns, consistent with a function on mRNAs, and on SUTs, CUTs, and NUTs. The TRAMP5 complex (Mtr4, Air1, Trf5) shows binding enrichment for introns, rRNAs, tRNAs, snRNAs, and snoRNAs. This is in agreement with previous data, which showed rRNA binding for Mtr4 and exosome subunits (Delan-Forino et al., 2017; Schneider and Tollervey, 2013). Moreover, the TRAMP complex cooperates with the Nrd1/Nab3 complex and the nuclear exosome complex during the maturation and 3' pre-processing of snoRNAs (Grzechnik and Kufel, 2008). To distinguish binding upon degradation and binding in order to pre-process snoRNAs, we investigated metagene profiles of TRAMP subunits along snoRNA genes (Appendix Figure 39). Air1/Trf5 bind almost exclusively to the gene body whereas Air2/Trf4 bind downstream of the $3^{\prime}$ end. This suggests that TRAMP5 is mainly involved in snoRNA degradation, whereas TRAMP4 may work together with the Nrd1/Nab3 machinery to pre-process snoRNAs (Appendix Figure 39) and to target NUTs, SUTs, and CUTs for degradation (Figure 8B).

The cross-linking preferences of subunits of the Ski complex differ only slightly from each other (Figure 8B, highlighted in cyan). All Ski complex subunits bind the $5^{\prime}$ UTR, CDS, and $3^{\prime}$ UTR of mRNAs, rRNAs, tRNAs, snoRNAs, and snRNAs. The Ski2 subunit preferentially binds to the CDS of mRNAs, consistent with its function as a helicase to detach bound proteins from the mRNAs (Houseley and Tollervey, 2009; Lebreton and Seraphin, 2008). The exosome adaptor subunit Ski7 preferentially binds rRNAs and tRNAs. These patterns are consistent with the model that the exosome cooperates with distinct accessory complexes and factors to target different transcript classes. Finally, we observed similar cross-linking patterns for all NMD factors with strong binding to SUTs and NUTs (Figure 8B, highlighted in yellow). Upf2 shows a binding preference to introns and CUTs. Upf3 also binds to the $5^{\prime}$ UTR, CDS, and 3' UTR of mRNAs, and Nmd4 binds to introns and 3' UTRs of mRNAs." 
A

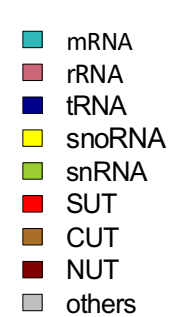

0

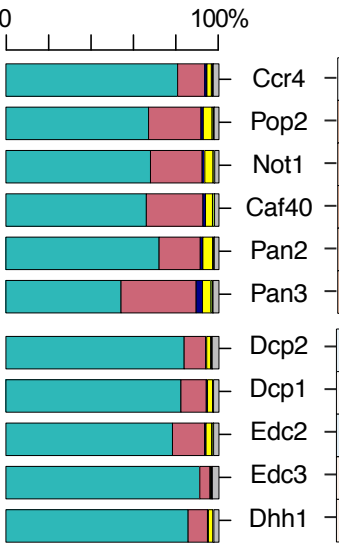

\begin{tabular}{|l||l|}
\hline & Xrn1
\end{tabular}

\begin{tabular}{|l|l||l|l|}
\hline & Rrp6 \\
\hline
\end{tabular}

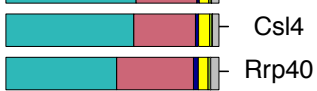

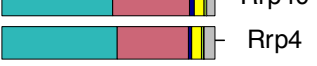

III- Rrp44

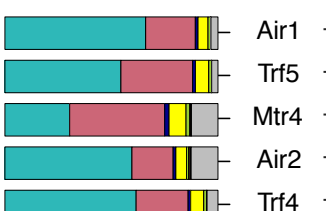

\begin{tabular}{|l|l||}
\hline & \\
\hline &
\end{tabular}

\begin{tabular}{|l||l}
\hline & \\
\hline &
\end{tabular}

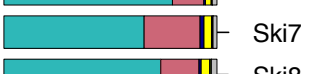

\begin{tabular}{l|l||l}
\hline & Ski8
\end{tabular}

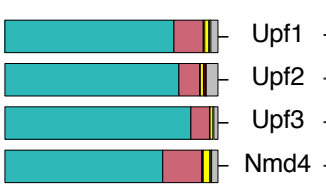

B
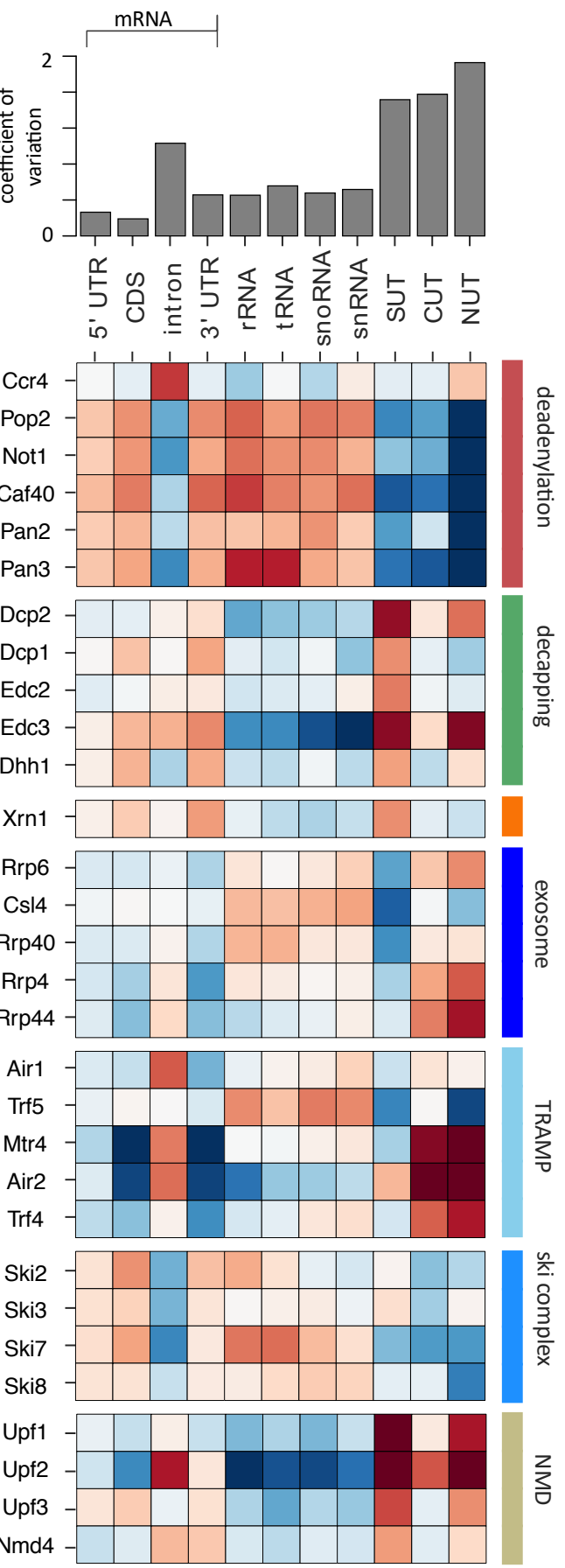

enrichment score

$\begin{array}{ccccc}1 & 1 & 1 & 1 & 1 \\ -2 & -1 & 0 & 1 & 2\end{array}$

Figure 8: Distribution of degradation factor cross-link sites over the yeast transcriptome.

A) Fractions of high-confidence PAR-CLIP sequencing reads of 30 yeast degradation factors fall into various transcript classes. Depicted classes are the following: messenger RNA (mRNA) in turquoise $(n=4,928)$, ribosomal RNA ( $r R N A)$ in antique pink $(n=24)$, transfer RNA (tRNA) in dark blue $(n=299)$, small nucleolar RNA (snoRNA) 
in yellow ( $n=77$ ), small nuclear RNA (snRNA) in green $(n=6)$, stable unannotated transcripts (SUTs) in red ( $n=357$ ), cryptic unstable transcripts (CUTs) in light brown $(n=750)$, Nrd1-dependent unterminated transcripts (NUTs) in dark brown $(n=317)$.

B) Enrichment z-scores of high-confidence PAR-CLIP cross-link sites of 30 yeast degradation factors (rows) in various segments of mRNA transcripts (left columns; UTR: untranslated region; intron; CDS: coding sequence), or other transcript classes as in A (other columns). The color-coded $z$-score is the fraction of binding sites of the factor $x$ in the transcript class $c$ minus the mean fraction for that transcript class $c$ divided by the standard deviation of fractions for class $c$ (color encoded, -0.8 in blue to 2.4 in red). The coefficient of variation on top is the standard deviation divided by the mean for each transcript class. Factors are grouped according to their functional role; from top to bottom: deadenylation machinery (Ccr4, Pop2, Not1, Caf40, Pan2, Pan3), decapping (Dcp2, Dcp1, Edc2, Edc3, Dhh1), Xrn1, exosome (Rrp6, Csl4, Rrp40, Rrp4, Rrp44), TRAMP polyadenylation complex (Trf5, Air1, Mtr4, Air2, Trf4), Ski complex (Ski2, Ski3, Ski7, Ski8), nonsense mediated decay (NMD) (Upf1, Upf2, Upf3, Nmd4).

\subsubsection{Distinct factor distribution along mRNA}

"We next focused on degradation factor distribution on mRNAs. We prepared metagene profiles showing the average occupancy of each factor around the mRNA transcription start sites (TSS) and poly-adenylation (pA) sites, respectively (Figure 9). The Pan2/Pan3 deadenylase complex and the Ccr4/Not subunits Pop2, Not1, and Caf40 all cross-link upstream of the $3^{\prime}$ end of mRNA with the highest enrichment at the pA site, as expected from their function in shortening the polyA tail. The catalytic subunit Ccr4 binds strongly in the $5^{\prime}$ region of mRNAs. All $5^{\prime}$ decapping factors bind upstream of the pA site, and all but the catalytically active subunit Dcp2 show increasing occupancy towards the $3^{\prime}$ end of mRNAs. These patterns can be explained if decapping factors are pre-bound to mRNAs that form a closed loop that holds the RNA ends in proximity. In contrast, Dcp2 binds almost exclusively at the pA site, suggesting that it might be recruited only upon active mRNA degradation. The cytoplasmic 5' exonuclease Xrn1 has the highest occupancy towards the $3^{\prime}$ end, similar to the previously published crosslinking and cDNA analysis (CRAC) data (Tuck and Tollervey, 2013), thereby resembling the binding profiles of the decapping factors. Comparison of the binding profiles aligned at the pA site or alternatively with profiles aligned at the translation stop codon shows that the binding preference indeed lies at the end of the 3' UTR independent of the stop codon position (Appendix Figure 29). 
The exosome core subunits (CsI4, Rrp40, and Rrp4) and the catalytically active subunits (nuclear: Rrp6, cytoplasmic: Rrp44) cross-link to the $5^{\prime}$ end of the transcript (Figure 9), possibly because the exosome binds to the $5^{\prime}$ end while digesting the $3^{\prime}$ end, or more likely because the exosome slows down towards the remaining $5^{\prime}$ end of mRNAs after rapid degradation from the $3^{\prime}$ end. Both TRAMP complexes bind mainly in the $5^{\prime}$ region of mRNAs near the TSS, as previously observed for Mtr4 and Trf4 (Tuck and Tollervey, 2013).

The Ski complex components Ski7 and Ski8 occupy the entire mRNA with increasing occupancy towards the pA site, whereas Ski2 and Ski3 show more discrete binding towards the polyA tail (Figure 9). The NMD factors Upf1 and Upf3 show binding over the entire mRNA with highest occupancy at the pA site, consistent with their role in scanning for premature stop codons in mRNAs and remodeling of the $3^{\prime}$ end of protein-RNA complexes and completion of mRNA decay (Franks et al., 2010). In addition, Upf2 and Nmd4 show strongest binding near the $3^{\prime}$ ends of mRNAs. Taken together, the distribution of crosslinks along mRNA transcripts differs between degradation complexes and in some cases also between their subunits." 


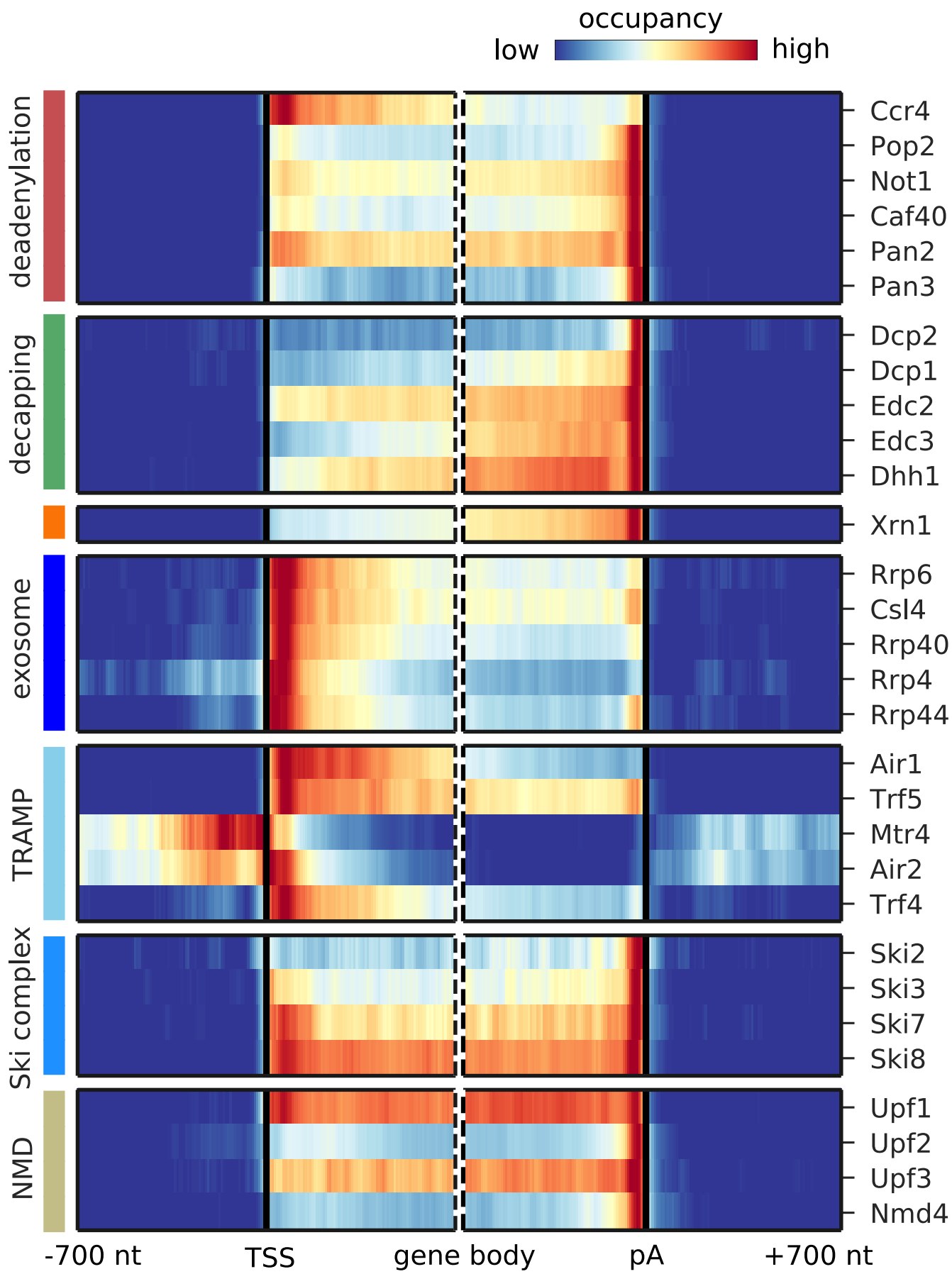

Figure 9: Metagene analysis of degradation factor binding on protein-coding mRNAs. Averaged occupancy profiles of degradation factors over mRNAs aligned around their transcription start site (TSS) $(n=3,193$, left) and around their poly(A) (pA) site $(n=3,193$, right) in a window of $[ \pm 700 \mathrm{nt}]$. Regions that have neighboring transcripts on the same strand were removed to avoid contaminating profiles (Methods). Factors are grouped according to their functional role; from top to bottom: deadenylation, decapping, Xrn1, exosome, TRAMP complex, Ski complex, and NMD. The color code shows the average occupancy normalized between the minimum and maximum values per profile (with high occupancy shown as dark red, and low occupancy shown in dark blue). 


\subsubsection{Surveillance of aberrant nuclear ncRNA}

"Pervasive transcription of the genome leads to many short-lived aberrant RNAs that must be rapidly detected and degraded in the nucleus. We previously reported that the RNA surveillance factors Nrd1 and Nab3 strongly cross-link to aberrant upstream antisense RNA that stems from bidirectional transcription (Schulz et al., 2013). In order to find factors cross-linking to aberrant ncRNAs, we plotted the occupancy of all 30 investigated factors on the antisense strand of known mRNAs (Figure 10). For comparison, we plotted the published Nrd1 and Nab3 profiles in the first two lanes of Figure 10. The factors involved in processing and degradation of Nrd1-unterminated transcripts, or NUTs (Schulz et al., 2013) are expected to show similar binding to upstream antisense RNA as Nrd1 and Nab3. Indeed, we observed a similar binding pattern for all exosome subunits (Rrp6, Csl4, Rrp40, Rrp4, Rrp44) and subunits of the TRAMP4 complex (Mtr4, Air2, Trf4). Consistent with this, these factors also bind strongly to previously annotated NUTs and CUTs (Figure 8). It has been shown that $\mathrm{Nrd} 1$ is involved in terminating transcripts upstream of the TSS. We also observe a strong signal for binding upstream of the TSS on the sense strand for Air2 and Mtr4 (Figure 9). This suggests that the TRAMP4 complex is involved in degradation of those Nrd1-regulated upstream sense transcripts. To investigate this hypothesis, we compared the binding profiles around the TSS of 459 protein coding genes, previously annotated as having upstream Nrd1-unterminated transcripts, or NUTs (Schulz et al., 2013)' with the profiles obtained for all mRNAs (Appendix Figure 40). TRAMP4 and the exosome subunits show a strong preference for binding to the upstream promoter region of the genes that are controlled by the Nrd1/Nab3 complex (Appendix Figure 40)."

"These results are consistent with the idea that the nuclear RNA surveillance machinery involves, in addition to Nrd1 and Nab3, the TRAMP4 complex and the nuclear exosome. Indeed, it was reported that TRAMP4 can add a short polyA tail on aberrant RNAs (Wyers et al., 2005), which may trigger degradation by the nuclear exosome. It was also recently shown that Nrd1 and Trf4 interact, providing a basis for coupling surveillance-mediated termination to RNA degradation (Tudek et al., 2014)." 


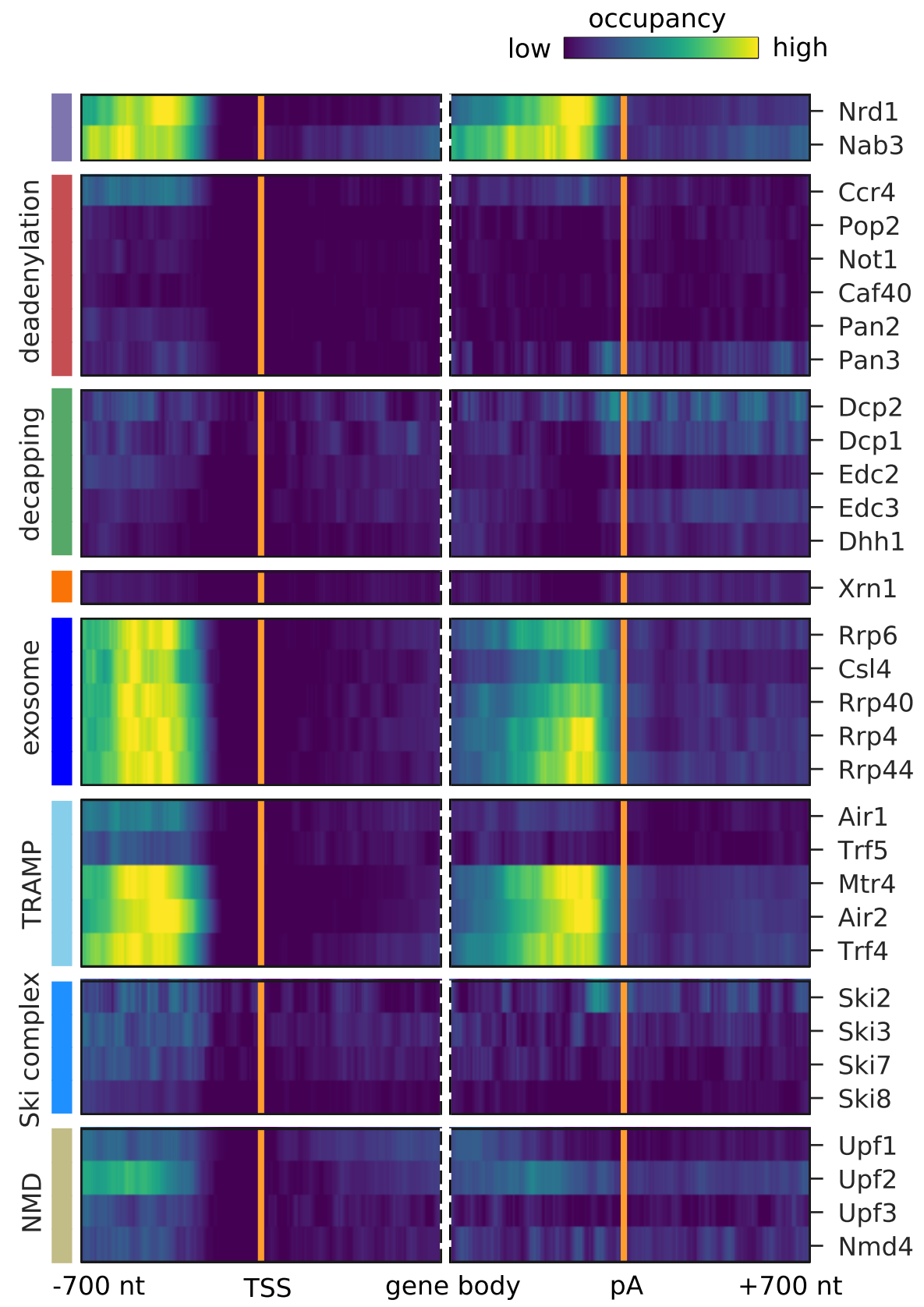

Figure 10: Surveillance of aberrant nuclear antisense RNAs by the exosome and the TRAMP4 complex.

Averaged occupancy profiles of degradation factors binding to transcripts antisense of mRNAs aligned around transcription start site (TSS) ( $n=3,076$, left) and around their polyadenylation $(p A)$ site $(n=2,705$, right) in a window of $[ \pm 700 n t]$. Regions with annotated genes on the antisense strand are removed to avoid contaminating the profiles (Methods). The color code shows the average occupancy normalized between the minimum and maximum values per profile (with high occupancy shown with yellow, and low occupancy in dark blue). On top, previously published PARCLIP profiles for Nrd1 and Nab3 are included for comparison (Schulz et al., 2013). 


\subsubsection{Interactions between RNA processing machineries}

"To find out which groups of factors can work together in degrading transcripts, we analyzed their tendency to co-occupy the same transcripts by calculating the Pearson correlation of their occupancy across all transcripts (Figure 11A). We also analyzed their co-localization, that is, the tendency of a factor to bind near to another factor's binding sites, using a range of $\pm 40 \mathrm{nt}$ from each cross-link site (Figure 11B). To relate these profiles to those of other factors, we included previously published PAR-CLIP profiles from our lab (Appendix Table 13). Profiles were available for factors that function in nuclear RNA surveillance (Nrd1, Nab3), cap binding (Cbc2), mRNA transcript elongation (Bur1, Bur2, Ctk1, Ctk2, Cdc73, Ctr9, Leo1, Paf1, Rtf1, Set1, Set2, Dot1, Spt5, Spt6, Rpb1), pre-mRNA splicing (Ist3, Nam8, Mud1, Snp1, Luc7, Mud2, Msl5), pre-mRNA 3' processing (Pab1, Pub1, Rna15, Mpe1, Cft2; Yth1), transcription termination (Rat1, Rai1, Rtt103, Pcf11), and mRNA export (Hrp1, Tho2, Gbp2, Hrb1, Mex67, Sub2, Yra1, Nab2, Npl3) (Baejen et al., 2017, 2014; Battaglia et al., 2017; Schulz et al., 2013).

A two-dimensional embedding of co-occupancy profiles between all these processing factors is shown in Figure 11C. It represents the degree of similarities between co-occupancy of transcripts (Figure 11A) in terms of the distance in two dimensions. The two-dimensional embedding of the colocalization matrix in Figure 11B shows a similar clustering. This extensive global analysis suggests which factors reside in functional complexes and which functional complexes may interact during RNA processing and degradation. The analysis recovers several established interactions between subunits of known complexes and between different complexes, providing a positive control. For example, all factors of the decapping complex show very high co-occupancy and co-localization, as do Air2 and Mtr4, which reside in the TRAMP4 complex.

The analysis contains a lot of new information, forcing us to focus here on a few interesting, novel findings (Figure 11C). First, the largest cluster is formed by the previously analyzed factors involved in transcription elongation by RNA polymerase $\|$ (cluster 1) and in co-transcriptional pre-mRNA processing, including cap-binding complex (Cbc2), 3'processing, transcription 
termination, and RNA export. The degradation factors Ccr4 and Air1 also reside in this cluster, maybe reflecting the role of $\mathrm{Ccr} 4$ in transcription elongation (Kruk et al., 2011). A second cluster is formed by splicing factors (cluster 2). Factors involved in nuclear and cytoplasmic exosomal degradation (Rrp6, Cs14, Rrp4, Rrp40 and Rrp44) form a third cluster (cluster 3). Close to cluster 3, we find the TRAMP4 complex subunit Trf4, the elongation factors Dot1, Paf1, Leo1, and the termination factors Pcf11 and Rai1. Rai1 has been shown to detect and remove incomplete $5^{\prime}$ cap structures, to subject aberrant pre-mRNAs to nuclear degradation (Jiao et al., 2010).

A forth cluster is formed by mRNA deadenylation factors together with pA tail binding proteins (Pab1 and Pub1), Ski7, Ski8, Trf5, and the export factor Yra1 (cluster 4). This is consistent with coupled mRNA deadenylation and subsequent degradation from its $3^{\prime}$ end by the exosome with the Ski or TRAMP complex as adaptors. The fifth cluster is formed by mRNA decapping factors, which cluster together with Xrn1, suggesting a coupling of mRNA decapping with degradation from the $5^{\prime}$ end by Xrn1 (cluster 5 ). The NMD-involved factors Upf1, Upf2, Upf3 and Nmd4, and Ski2 and Ski3 are also found in cluster 5. The high correlation between Xrn1 and Ski2 has been reported in a CRAC experiment (Tuck and Tollervey, 2013). The elongation factor Ctr9, the 3'processing factor Mpe1 and the export factors Tho2, Mex67 and Nab2 are also found in cluster 5 . A last cluster (cluster 6 ) is formed by factors involved in nuclear RNA surveillance, including Air2, Mtr4 and the Nrd1/Nab3 complex. Taken together, these findings are consistent with known functional associations and physical interactions between factors and suggest intriguing new associations to be investigated in future work." 
A
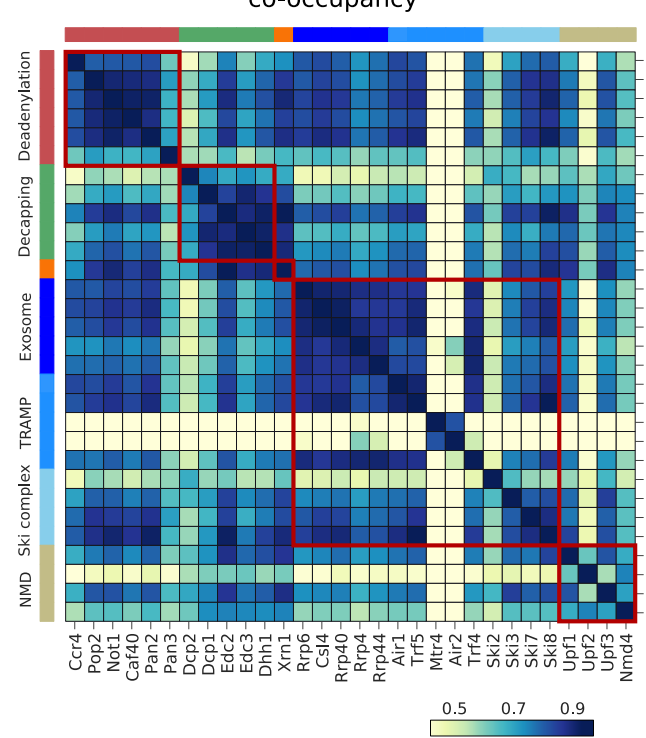

B

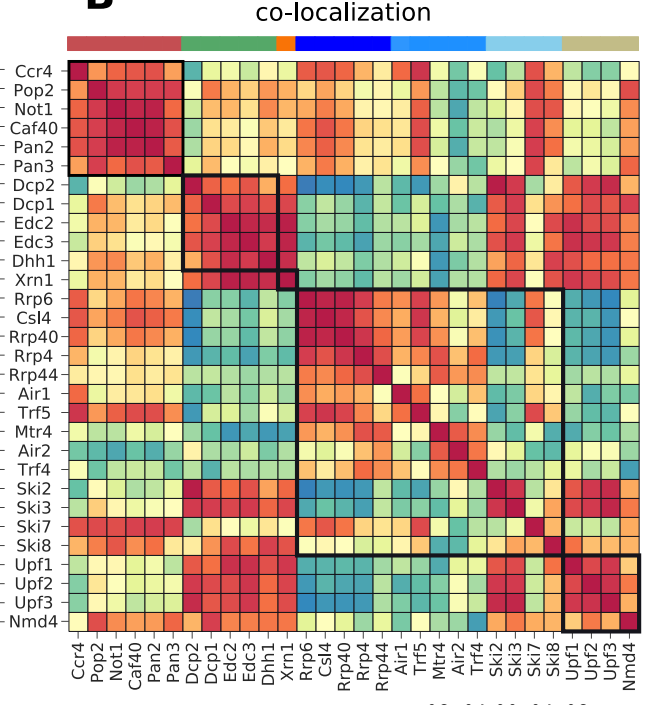

$\begin{array}{lllll}-0.8 & -0.4 & 0.0 & 0.4 & 0.8\end{array}$

C

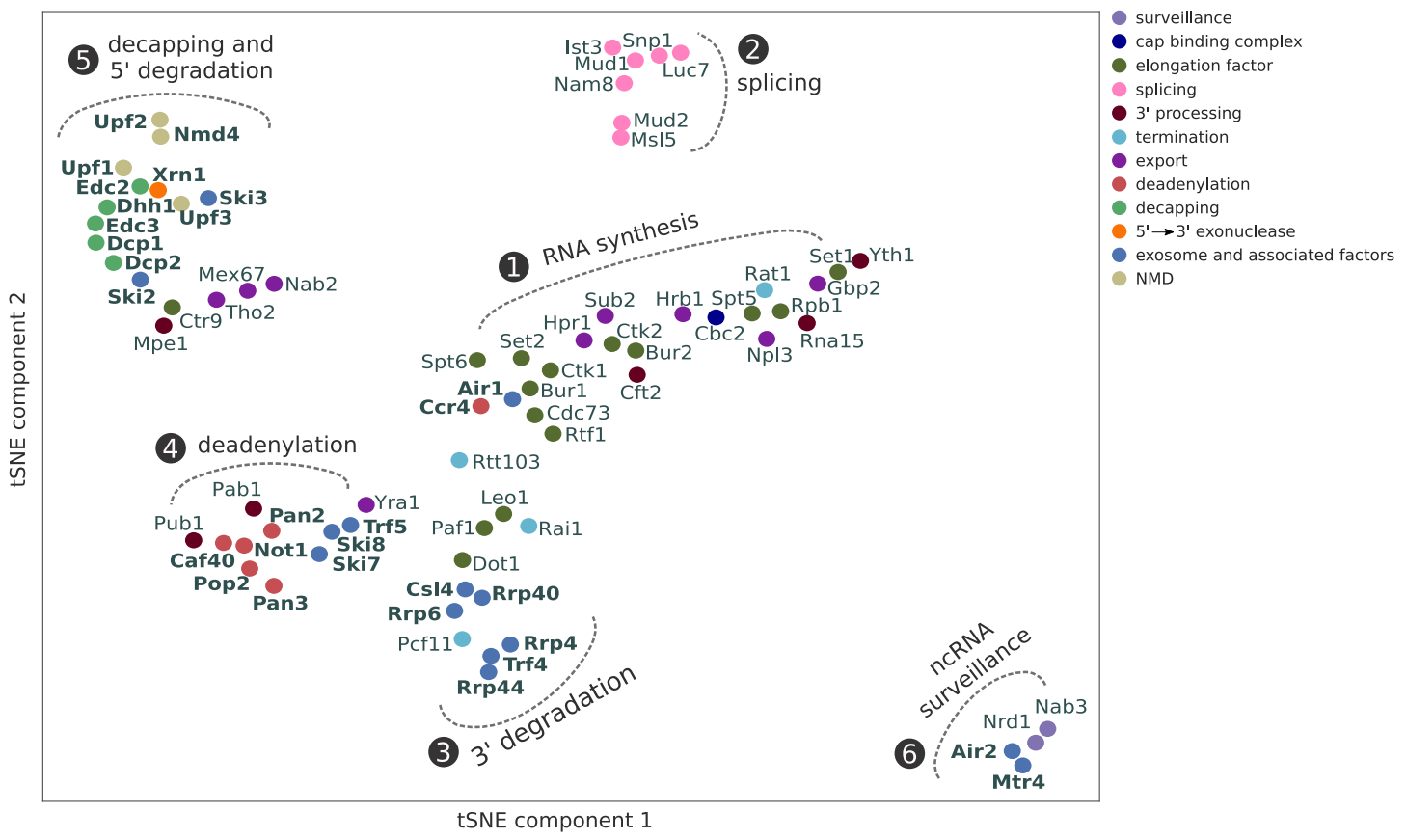

Figure 11: Global co-occupancy and co-localization analysis reveals unexpected cooperation between factors from different complexes and pathways.

A) Matrix of pairwise correlation coefficients of factor occupancies evaluated over all transcripts. B) Matrix of co-localization based on the enrichment of factor $x$ binding within $40 \mathrm{nt}$ upstream and downstream of the cross-link site of factor $\mathrm{x}^{\prime}$. C) Twodimensional embedding of the co-occupancies in A) analyzed for 74 RNA processing factors with tSNE, including 30 factors from this study (highlighted in bold), and 44 factors from previous studies (Baejen et al., 2014, 2017; Battaglia et al., 2017; Schulz et al., 2013). Factors that are plotted in close proximity show a preference for binding to the same transcripts. Clusters present factors involved in RNA synthesis (1), splicing (2), 3' processing (3), deadenylation (4), decapping (5), and surveillance (6). 


\subsection{5 $5^{\prime}$ degradation machinery senses translation efficiency}

"To study the link between cytosolic mRNA translation and degradation, we compared the occupancy of degradation factors on mRNAs to their average codon-optimality score ('transcript optimality') (Figure 12A, Appendix Figure 31Figure 37). We found that the $5^{\prime}$ decapping machinery and Xrn1 preferentially bind transcripts with low transcript optimality. In contrast, the $3^{\prime}$ deadenylation machinery and the exosome bind more strongly to optimal transcripts. We asked whether this correlation with codon optimality is introduced by only a few differentially bound codons or by global enrichment/depletion of optimal codons. For this purpose, we introduced a 'codon enrichment score', which measures a codon's enrichment in the set of transcripts bound by the factor relative to the yeast mRNA pool. For Dcp2 this enrichment score is high on nonoptimal codons, and low on optimal codons, whereas the opposite trend is observed for Ccr4 and most degradation factors (Figure 12B, Appendix Figure 31-Figure 37). This is consistent with a model that ribosome stalling on translationally inefficient codons can lead to recruitment of Dcp2 and Xrn1 and subsequent $5^{\prime}$ degradation of the transcript (Heck and Wilusz, 2018).

To investigate the significance of the correlation between transcript optimality and binding of the $5^{\prime}$ degradation machinery, we compared the contribution of several mRNA features in explaining the occupancy patterns retrieved from PAR-CLIP experiments. Since mRNA expression, half-life, and translation optimality are inter-correlated (Appendix Figure 30), a causative effect of one of these features on binding strength may lead to correlations with all three features. To better distinguish correlation from causation, we used linear regression analysis to explore whether correlations between factor binding and optimality are better explained with other mRNA features (Appendix Figure 38). We assessed the significance of features via the likelihood ratio test on the multi-variate linear regression model for occupancy. The likelihood ratio test calculates the significance of a feature from the change of the likelihood (quantifying the prediction quality) upon removal of that feature from the regression model. For decapping enhancers (Edc2, Edc3, and Dhh1) and Xrn1, low codon optimality is the most determining feature for binding (Figure 12C). The same is true for NMD factors Upf1 and Upf3, which are 
A

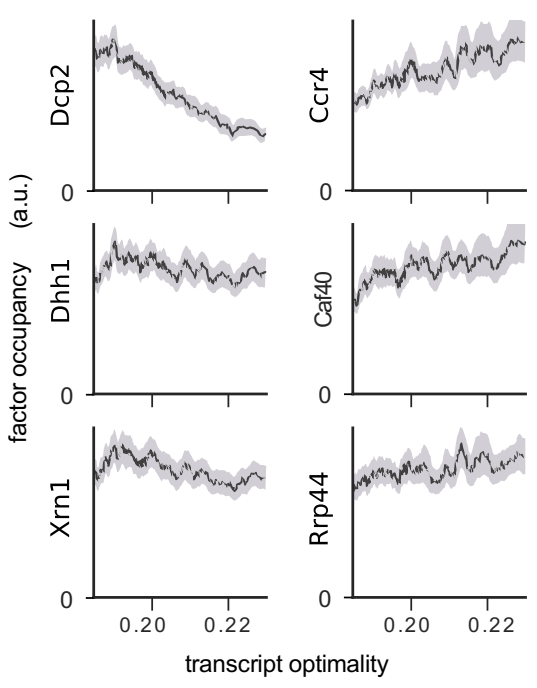

B

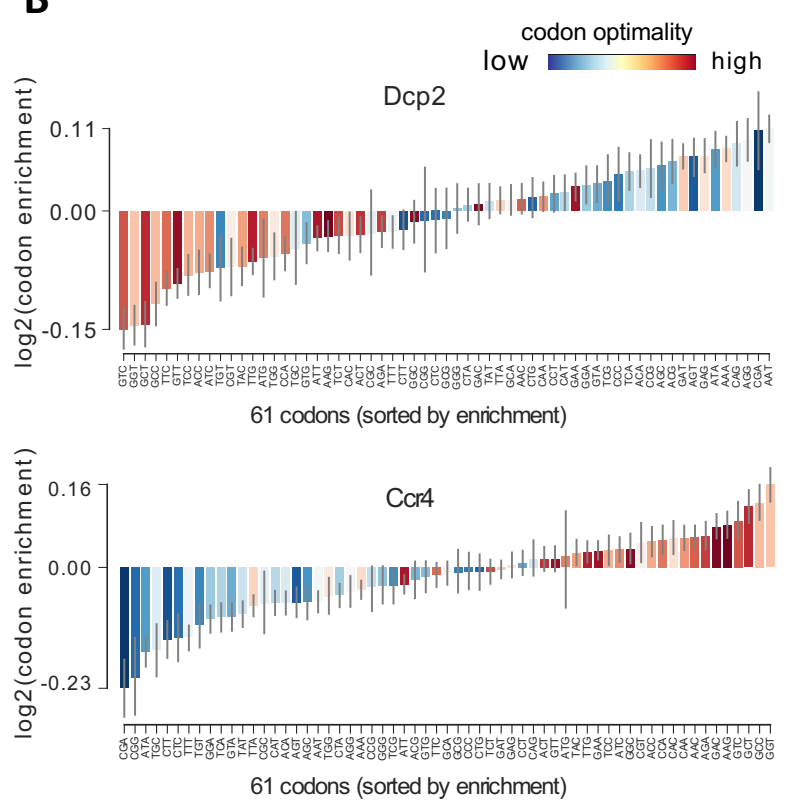

C

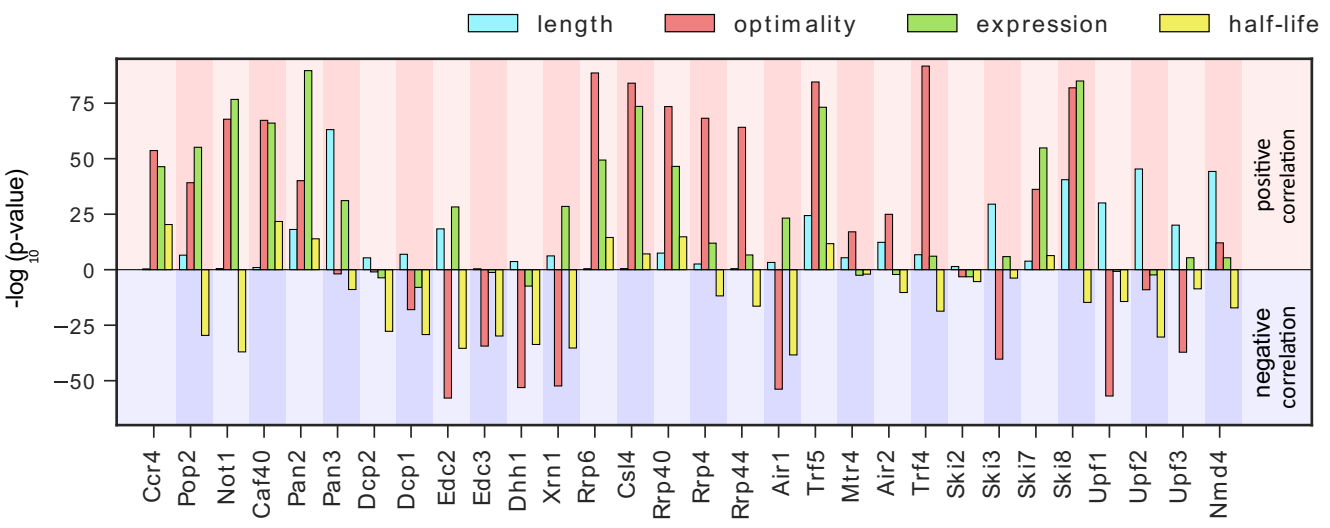

Figure 12: Binding preferences reveal a link between decapping-mediated degradation and translation.

A) Total occupancy per mRNA (according to TIF-seq annotation) for six factors as a function of the average mRNA codon optimality (transcript optimality). The occupancy of factors from the $5^{\prime} \rightarrow 3^{\prime}$ degradation machinery (decapping and Xrn1, left) decreases with increasing transcript optimality, whereas the occupancy of factors from the $3^{\prime} \rightarrow 5^{\prime}$ degradation machinery (Ccr4, Caf40 and exosome subunit Rrp44) increases with increasing average codon optimality. (Grey shading: 95\% confidence intervals generated by bootstrapping mRNAs). B) Codon enrichment in transcripts bound by Dcp2 and Ccr4 compared to the average frequency over all mRNAs. The bar colors represent codon optimality, with highly optimal codons shown in dark red. (Thin grey lines: $90 \%$ confidence intervals generated by bootstrapping coding sequences.) C) Significance of correlations between the binding strength of degradation factors and transcript length, transcript optimality (Pechmann and Frydman, 2013), expression level (Baejen et al., 2017), and half-life derived by multivariate linear regression analysis (Methods). Bars are separated according to the direction of correlation with positive correlation marked by a red background and negative correlation marked by a blue background. 
known to bind non-optimal transcripts (Celik et al., 2017). This result confirms the importance of the translation efficiency for the stability of cytosolic mRNAs and strengthens our finding that transcripts with low average codon optimality are preferentially targeted by the decapping machinery and degraded from the 5' end."

\subsubsection{Decapping factors are enriched upon RNA degradation}

"Although decapping occurs at the 5 ' end of mRNAs, decapping factors show a strong occupancy near the 3 ' end (Figure 9). To investigate this further, we compared metagene profiles of decapping factors between stable (top 25\%) and unstable (bottom 25\%) transcripts, using mRNA half-life estimates (Figure 13A, Methods). On both stable and unstable mRNAs, Dcp1, Edc2, Edc3, and Dhh1 show increased binding near the $3^{\prime}$ end, but unstable RNAs show a higher occupancy in the transcript body. The catalytically active subunit Dcp2 binds almost exclusively at the $3^{\prime}$ end and has a higher occupancy on unstable transcripts. Moreover, A-rich 4-mers are abundant around the proximity (8 nt) of Dcp2-cross-link sites (Figure 13C), indicating a binding preference of Dcp2 for A-rich RNA sequences. Overall, these binding patterns suggest that decapping factors are bound in transcript bodies and near the $3^{\prime}$ end of transcripts, and that through closed-loop formation of the mRNA they are in close proximity to the $5^{\prime}$ end. Decapping factors might also travel with the $5^{\prime} \rightarrow 3^{\prime}$ exonuclease Xrn1 upon RNA degradation.

Decapping factors may bind to complete mRNAs or to transcripts that are in the process of being degraded. To quantify these two behaviors, we combined our PAR-CLIP occupancy data with RNA half-life estimates (Methods). We modeled the occupancy of factors on mRNA as the sum of binding to all transcripts (b) and surplus binding to transcripts that are in the process of degradation $\left(\frac{\mathrm{a}}{\mathrm{t}_{1 / 2}}\right)$. Therefore, we can model occupancy as a function of half-life with a linear equation (occupancy $=\frac{a}{t_{1 / 2}}+b$ ). In cases where there 
A

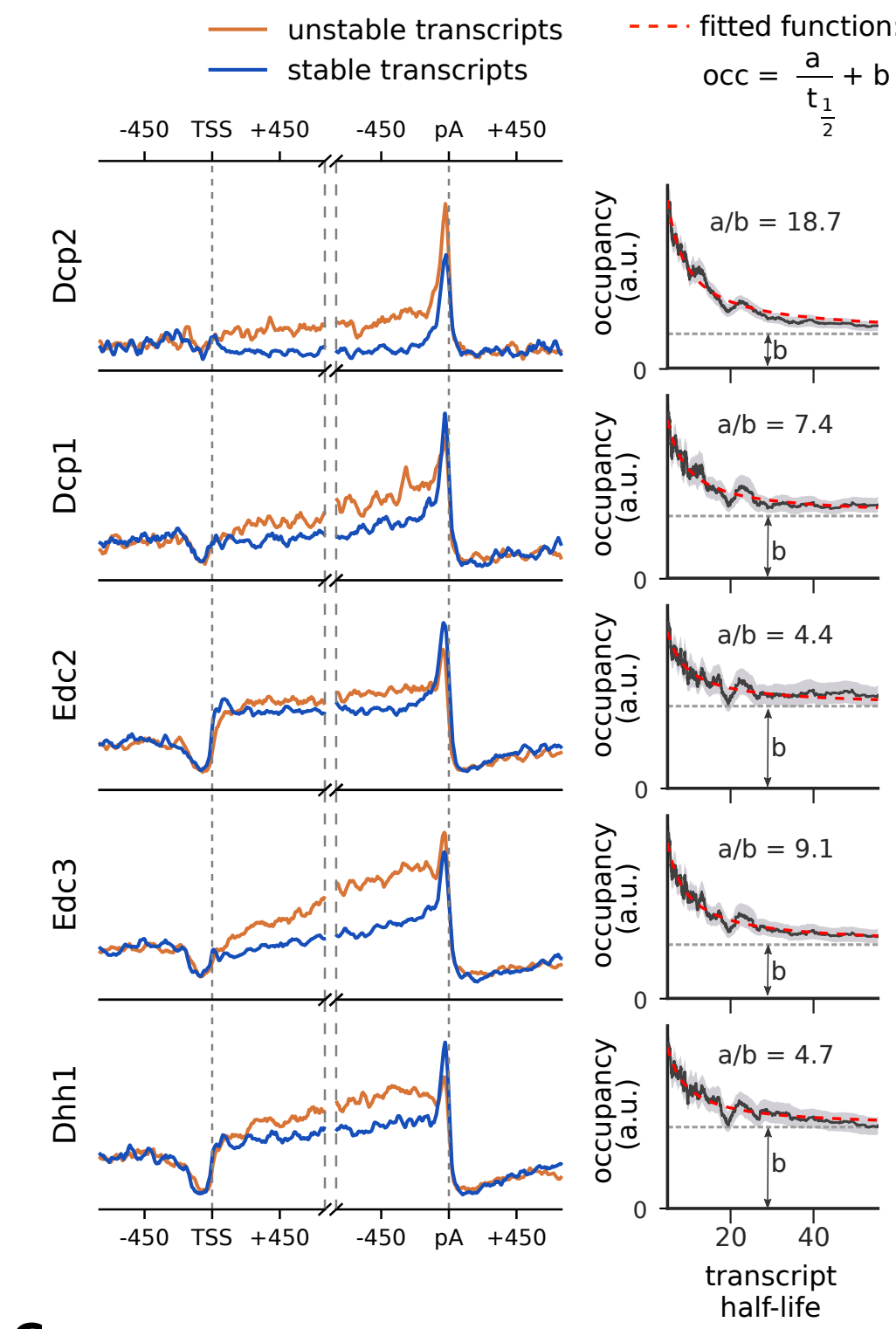

C

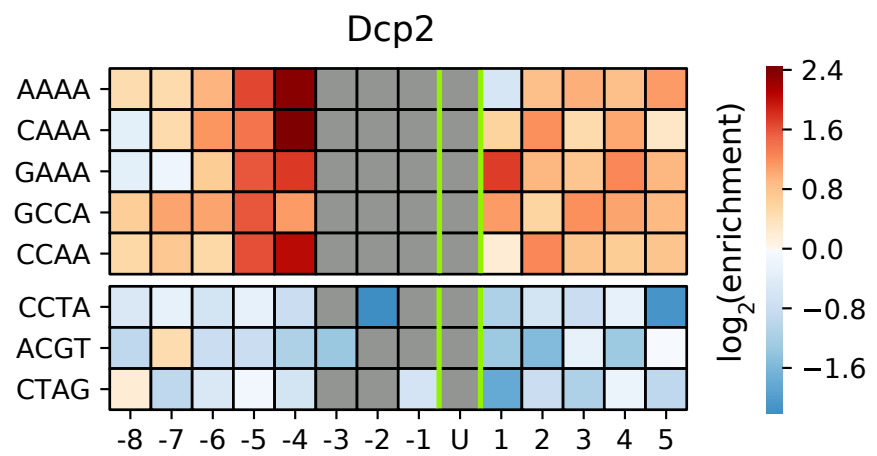

Figure 13: Location and recruitment of the decapping complex Dcp1/Dcp2 and decapping enhancers Edc3, Dhh1, and Edc2.

A) Smoothed, transcript-averaged PAR-CLIP occupancy profiles aligned at TSS and pA sites $[ \pm 750 \mathrm{nt}]$ of unstable and stable transcripts (first and fourth quantile of half-life 
distribution, respectively). B) Dependence of total occupancy of factors on the transcripts half-life. The fitting function is plotted in red and the fitted value for $b$ is marked with a dashed gray line. (Grey shade: 95\% confidence intervals generated by bootstrapping transcripts). C) Sequence binding preference for the catalytically active subunit of decapping complex (Dcp2), illustrated with the 5 most enriched and the 3 most depleted 4-mers. The color code shows the log2 enrichment factor of 4-mers around PAR-CLIP cross-link sites $[ \pm 5 \mathrm{nt}]$. Dark red represents strong enrichment and dark blue shows strong depletion of a 4-mer. Infeasible combinations are shown with grey. The most highly enriched field is binding AAAAU with the cross-link at the $U$, which is enriched over random expectation approximately $2^{3}=8$-fold.

is no surplus binding upon active degradation, i.e., the occupancy is the same as in intact RNAs, 'a' will be zero. For 5' decapping factors, this model closely fits the occupancy patterns retrieved from our experiments (Figure 13B), other degradation factors also follow this pattern to varying degrees (Appendix Figure 31-Figure 37). In particular, Dcp2 shows a very high a/b ratio, revealing that it cross-links preferentially to transcripts that are being degraded. This analysis strongly suggests that the $5^{\prime}$ decapping machinery, although present to some extent on complete mRNAs, is enriched when mRNAs are degraded." 


\subsection{Discussion and Outlook}

4.2.1 Occupancy profiles of general eukaryotic degradation factors give new insights into RNA degradation processes

In this work we generated transcriptome-wide binding maps for 30 RNA degradation factors in the yeast $S$. cerevisiae. A detailed bioinformatics analysis of the NGS data revealed how degradation factors vary in their binding specificities for different classes of RNAs (Figure 8) and with respect to their preferred locations on RNA transcripts (Figure 9 and Figure 10). Global comparisons of the degradation factor profiles with previously published transcriptome-wide binding maps of other RNA-binding factors (Baejen et al., 2017, 2014; Battaglia et al., 2017; Schulz et al., 2013) revealed factors that cooccupy RNAs or co-localize on RNAs thereby forming clusters (Figure 11). Our data are consistent with published literature as discussed below and extend these findings on mostly single genes to a genome-wide scale. In addition, our analysis revealed several unexpected, novel insights, which we also discuss here. Although our data reflect factor binding signal and measure occupancy on transcripts, and do not directly reveal the function of factors, the correlations of occupancies between factors and with transcript properties indicate functional aspects and suggest functional associations between factors. We provide speculations on possible functional implications of our findings that can guide future studies.

\subsubsection{Initial step of RNA decay - mRNA deadenylation}

The first step of cytoplasmic mRNA decay is deadenylation of the polyA tail (Cao and Parker, 2003; Parker and Song, 2004). We have analyzed binding of Ccr4-Not and Pan2-Pan3 deadenylation complexes to RNAs by PAR-CLIP and observed similar binding patterns regarding RNA specificity (Figure 8). For the Pan2 subunit we observed cross-link sites throughout the mRNA (Figure 9) suggesting a function of Pan2 over the entire transcript such as scanning for degradation marks. For both subunits of the Pan2-Pan3 complex the occupancy is at the maximum close to the pA site (Figure 9). For the Ccr4-Not 
complex we observed RNA binding more towards the $3^{\prime}$ end of transcripts, except for Ccr4, which shows stronger cross-linking to the $5^{\prime}$ end (Figure 9). It has been proposed that the Pan2-Pan3 complex initiates deadenylation of the polyA tail followed by the Ccr4-Not complex, which acts on the shortened tail (Beilharz and Preiss, 2007; Brown and Sachs, 1998; Tucker et al., 2001). In agreement with this hypothesis, we observed binding of deadenylation complexes at the pA site (Figure 9). One limitation of the PAR-CLIP technique is that factor binding to the polyA tail cannot be resolved. This is due to the inability to map the polyA tail as it is not encoded in the reference genome. Therefore, we are not able to detect binding of the deadenylation factors within the polyA tail directly. Our method only allows for mapping of factors located close to the pA site. With this information we cannot elucidate the order of events during deadenylation.

In addition to the pA site binding of deadenylation factors, we observed binding of the deadenylase Ccr4 to the $5^{\prime}$ end of mRNAs (Figure 9), which indicates a different or additional function of this protein as previously suggested (Miller and Reese, 2012). This specific binding pattern and its colocalization with transcription elongation factors (Figure 11) can also reflect its function in transcription elongation by RNA Pol II (Kruk et al., 2011). During deadenylation, it has been shown that Pab1 interacts with the Ccr4-Not complex, thereby stimulating deadenylation activity and differentiating the roles of the nuclease enzymes Ccr4 and Pop2 (Webster et al., 2018). By using a fully reconstituted biochemical system with proteins from the fission yeast Schizosaccharomyces pombe, the authors showed that Pab1 release depends on Ccr4 activity. In vivo experiments in S. cerevisiae showed that Ccr4 acts on all mRNAs and thus, is a general deadenylase. Our PAR-CLIP data revealed Ccr4 occupancy at the $5^{\prime}$ end of mRNAs (Figure 9) indicating that its binding to mRNAs is independent of the polyA tail and Pab1. In addition, we observed enriched binding of Ccr4 within introns (Figure 8). This leads us to the speculation that Ccr4 could be recruited to the mRNA $5^{\prime}$ end or intronic sequences to release Pab1 from the polyA tail and further its deadenylation. In contrast to Ccr4, Pop2 has been found to just trim the polyA tail, which is not protected by Pab1 (Webster et al., 2018). In agreement with this, we were able 
to detect Pop2 binding at the pA site (Figure 9) probably due to the absence of Pab1.

The authors concluded based on their data that Pop2 is a specialized enzyme deadenylating selected transcripts characterized by reduced Pab1 occupancy and lower translation elongation rates (Webster et al., 2018). Lower translation elongation rate indicates Pop2 binding to transcripts with nonoptimal codons. In contrast, we observed a minor binding preference of Pop2 on mRNA with optimal codons (Appendix Figure 31). This inconsistency of data may be explained by the usage of two different experimental settings: a whole cell approach for our PAR-CLIP experiments, while the other experiment was performed in an isolated and biochemically reconstituted system. In contrast to Pop2, Ccr4 is bound preferentially to codons with high optimality (Figure 12) suggesting that deadenylation and degradation initiation of the mRNA are independent of a high translational efficiency.

\subsubsection{Decapping as first step for RNA degradation from the 5' end}

Following deadenylation, decapping is the next step in cytosolic mRNA degradation (Franks and Lykke-Andersen, 2008; Tharun and Parker, 2001). In our study, we observed binding of decapping factors primarily towards the polyA tail (Figure 9), and enriched cross-link sites on mRNAs and non-coding SUTs (Figure 8). The preferred localization of $5^{\prime}$ decapping factors near the $3^{\prime}$ end and $3^{\prime}$ degradation factors close to the $5^{\prime}$ end seems counterintuitive. Gallie et al. explained this phenomenon by the model of an mRNA closed-loop structure due to messenger ribonucleoprotein (mRNP) formation, in which the $5^{\prime}$ cap is in close proximity to the polyA tail (Gallie, 1991). The decapping factors can be deposited near the $3^{\prime}$ end of transcripts and upon polyA tail shortening, the decapping complex is activated. This leads to decapping and subsequent rapid degradation of the transcript by the $5^{\prime} \rightarrow 3^{\prime}$ exonuclease Xrn1. In this model, decapping opens the RNA closed-loop structure, allowing access for Xrn1 and thereby enabling RNA degradation. Supporting this model, we determined that the catalytically active subunit Dcp2 shows the highest activity 
on unstable transcripts compared to decapping activators (Figure 13). Our data suggest that decapping enhancers can be pre-bound to stable mRNAs waiting for the recruitment of the catalytic subunit (Figure 13).

Furthermore, we observed higher enrichment of decapping factors on translationally inefficient codons (Figure 12). This agrees with previous findings that suggested a link between RNA decay and translation (reviewed in Huch and Nissan, 2014). This link is thought to be established by the decapping enhancer Dhh1 sensing ribosome velocity. Ribosomes are slowed down on non-optimal codons, which reduces the translation rate and this can lead to activation of deadenylation and decapping (Radhakrishnan et al. 2016).

We observed further cross-link sites for Xrn1 throughout the transcript with higher occupancy towards the $3^{\prime}$ end (Figure 9). Binding at the pA site has been observed for Xrn1 before using a similar approach (Tuck and Tollervey, 2013). This binding pattern may be explained by slower decay towards the transcript 3'end allowing for higher detection.

\subsubsection{Nuclear RNA surveillance mechanism}

Genomes of eukaryotic cells are pervasively transcribed with about $85 \%$ of the yeast genome giving rise to RNA transcripts, although only a few percent correspond to protein-coding mRNAs (David et al. 2006). In yeast, pervasive transcription stems from bidirectional Pol II transcription initiation of two opposing pre-initiation complexes within a nucleosome free region over the promoter region (Murray et al., 2012; Rhee and Pugh, 2012). The work from our laboratory has previously shown that the Nrd1-Nab3 surveillance machinery selectively terminates ncRNA synthesis, including transcripts synthesized in antisense direction to annotated genes and divergent transcription from bidirectional promoters (Schulz et al. 2013). With global mapping of degradation factors and in comparison to the metagene profiles of Nrd1-Nab3 (Schulz et al., 2013), we are able to propose a mechanism on how the surveillance machinery degrades antisense RNA in order to protect the cell from aberrant ncRNAs after early transcription termination. Several lines of evidence such as enrichment of these complexes on NUTs (Figure 8), similar 
binding profiles (Figure 10), as well as transcript co-occupancy analysis (Figure 11) suggested that after recognition through the sequence specific Nrd1-Nab3 complex, the ncRNA is polyadenylated by the TRAMP4 complex. The interaction of the Nrd1-Nab3 complex and Trf4 subunit of the TRAMP4 complex has previously been shown by our group and colleagues (Tudek et al., 2014). The short polyA tail can then mark the RNA for degradation by the nuclear exosome. This mechanism is likely to be the same for regulation of introns and ncRNAs upstream of mRNAs on the same strand, which were annotated as NUTs and CUTs, for which we see similar occupancy of the same degradation factors (Figure 8 and Figure 40). Our results indicate that the degradation machinery for all short-lived ncRNAs in the nucleus requires the same degradation factors such as Nrd1-Nab3, TRAMP4 and the nuclear exosome.

4.2.5 RNA degradation by the exosome complex and auxiliary factors

The second major cytoplasmic mRNA degradation pathway in the $3^{\prime} \rightarrow 5^{\prime}$ direction of transcripts involves the exosome complex (Zinder and Lima, 2017). In addition to mRNA decay, the exosome also targets long-lived transcripts such as tRNAs, rRNAs, snoRNAs, and snRNAs for processing and decay (Zinder and Lima, 2017). Our observations regarding the different binding patterns of the exosomal subunits and co-factors (Figure 8 and Figure 9) support the hypothesis that these factors are needed for RNA processing and degradation specificity (Delan-Forino et al., 2017). It has been proposed that exosome subunit localization regulates RNA degradation targets (Vanacova and Stefl, 2007). The RNA transcript can be channeled through the exosome core (Rrp4, Rrp40 and Cs14) towards the catalytic subunit Rrp44 (Kowalinski et al., 2016). This suggests that all factors have direct RNA contacts, which we were able to support by observing extensive crosslinking to mRNAs and many other non-coding transcripts (Figure 8). All exosome subunits cross-link towards the $5^{\prime}$ end of mRNAs (Figure 9). This unexpected observation might be caused by the exosome moving rapidly from the $3^{\prime}$ to the $5^{\prime}$ end and then 
residing at the $5^{\prime}$ end for a longer time, enabling for extensive cross-linking and thus detection.

The exosome co-factor Ski2 and the other subunits of the Ski complex show strong cross-linking at the pA site of mRNA (Figure 9). This indicates that the Ski complex is necessary during initial steps of RNA degradation. This can be explained by the helicase activity of Ski2, which dissolves RNA secondary structures and detaches proteins from the RNA allowing the exosome to degrade the transcript from the $3^{\prime}$ end (Schneider and Tollervey, 2013).

The TRAMP complexes are additional exosome co-factors and show binding to mRNAs towards the $5^{\prime}$ end of transcripts similar to the core and catalytic subunits of the exosome (Figure 9). This indicates that TRAMP complex mediated targeting of defective nuclear mRNAs and aborted transcription products for exosomal degradation might be similar to the Ski complex in the cytoplasm. The helicase Mtr4 may have a similar function as the Ski2 helicase in dissolving the secondary structure of the RNA and disassembling protein complexes bound to RNA (Falk et al., 2014). Additionally, TRAMP4 binding extended upstream of the TSS (Figure 9). This suggests targeting of non-coding transcripts emerging upstream of promoters for degradation. We have shown that this signal stems from previously annotated transcripts under attenuation control by Nrd1-Nab3 (Schulz et al., 2013), the TRAMP complex and the nuclear exosome (Appendix Figure 40). Moreover, it has been shown for the TRAMP complexes that they function in 3' end processing and degradation of snoRNAs (Grzechnik and Kufel, 2008; Losh et al., 2015). However, it has not been fully elucidated whether the complexes share the functions or distinguish between them. Using metagene profiles on snoRNAs, we were able to distinguish the functions of the TRAMP complexes (Appendix Figure 39). We propose that the TRAMP4 complex due to its binding downstream of the $3^{\prime}$ end of snoRNAs functions mainly in $3^{\prime}$ end processing. Binding of the TRAMP5 complex within the gene body of snoRNAs suggest a function mainly in its degradation. 


\subsubsection{Cytoplasmic RNA surveillance by NMD factors}

The NMD factors have a function in scanning for premature stop codons in mRNAs, remodeling of the $3^{\prime}$ end of protein-RNA complexes and completion of mRNA decay (Franks et al., 2010). This is consistent with our observation that Upf1 and Upf3 bind to the entire length of the mRNAs and with highest occupancy at the pA site (Figure 9). Moreover, Upf2 and Nmd4 showed the strongest binding near the $3^{\prime}$ ends of mRNAs close to endogenous stop codons. This indicates an additional role for NMD factors in recognition of the translation termination site.

The NMD-involved factors Upf1, Upf2, Upf3 and Nmd4 reside in a cluster together with mRNA decapping factors and the $5^{\prime} \rightarrow 3^{\prime}$ exonuclease Xrn1 (Figure 11C). This indicates that recognition of premature stop codons by the NMD machinery is coupled with initiation of decapping and degradation from the $5^{\prime}$ end by Xrn1. It has been shown that the NMD machinery is directly linked to the decapping complex via an adapter protein (Cho et al., 2009; Lai et al., 2012). Furthermore, we showed binding for the NMD factors Upf1 and Upf3 to non-optimal codons (Figure 12C). This is consistent with previous findings that NMD factors bind to non-optimal transcripts (Celik et al., 2017). This result confirms the importance of NMD factor binding for the stability of cytosolic mRNAs.

Furthermore, unannotated transcripts, which were predicted to lack protein-coding capacity in yeast, have been shown to be translated and targeted for degradation by the NMD pathway (Smith et al., 2014). Consistently with this observation, we observed binding of the NMD factors on SUTs (Figure 8). In addition to cross-linking sites in SUTs, we detected binding of the NMD machinery to unstable ncRNAs such as NUTs (Figure 8). This unexpected finding indicates that some NUTs escape degradation in the nucleus by the Nrd1-Nab3, TRAMP and exosome complex using an unknown mechanism. After export of the NUTs to the cytoplasm they might be targeted for translationdependent degradation via the NMD pathway. 
4.2.7 Perspectives of mechanistic insights into the RNA degradation process by complementation of the transcriptome maps with functional studies

We provide binding profiles of 30 RNA processing and degradation factors as an extensive resource that gives novel insights into eukaryotic RNA metabolism. The findings of this study provide a better understanding of nuclear degradation of short-lived ncRNAs. In addition to regulation of mRNA degradation by decapping and Xrn1-mediated $5^{\prime} \rightarrow 3^{\prime}$ decay mainly on translation inefficient codons. Several questions remain to be answered. Further biochemical experiments are required to study and prove the indications of functional interactions of short-lived ncRNAs and the involved degradation machinery. The proposed slowing down of the different degradation machineries (exosome complex and Xrn1) towards either end of the transcript needs to be investigated with kinetic measurements in vivo using single molecule techniques or in vitro with a fully reconstituted system to confirm our hypotheses. Further research needs to be undertaken to get a more detailed understanding of how the different long-lived ncRNAs such as tRNAs, rRNAs, snoRNAs, and snRNAs can be processed and finally degraded by the same exosome complex with different nuclear and cytoplasmic accessory factors. Structural analysis of the different exosome components and accessory proteins with their target RNAs might help to answer this question as the structures of the exosome and accessory factors alone cannot answer all questions (Falk et al., 2014; Halbach et al., 2013; Schmidt et al., 2016). In conclusion, the findings of this study provide several important insights and establish a basis for future research.

4.2.8 Protein-RNA binding studies of eukaryotic degradation factors in response to stress conditions will allow for better functional characterization of the RNA degradation machinery

Yeast cells undergo major metabolic adaption processes when they encounter stress situations, such as changes of the nutrient environment. To 
conserve energy, cells shut down gene expression of growth-related genes rapidly. At the same time, they increase expression levels of stress response and other metabolic genes. This leads to genome-wide changes in transcription and translation. Transcription levels are drastically reduced and translation is almost abolished in starved yeast cells (Ashe et al., 2000; Jona et al., 2000). At the same time, cytoplasmic degradation levels are also reduced and mRNAs are stabilized at a stage previous to polyA tail shortening (Jona et al., 2000). Down-regulation of growth-related genes in the nucleus is controlled by the Nrd1-Nab3-Sen1 and the TRAMP complexes targeting these genes for exosomal degradation (Bresson et al., 2017). Other genes like stress response genes escape degradation by decreased binding of Nrd1-Nab3 and TRAMP complexes, thereby resulting in upregulation of transcription. These findings have been obtained by genome-wide binding profiles of the Nab3 and Mtr4 proteins (Bresson et al., 2017). In addition, it has been observed that deadenylation by Ccr4 and Pan2 is inhibited in order to retain the majority of the cytoplasmic mRNA pool for later reuse after stress recovery (Hilgers et al., 2006). Nevertheless, how cytoplasmic degradation factors respond to nutrient changes has not been shown genome-wide. RNA binding studies using PARCLIP, in response to different stress conditions and in combination with RNAseq measurements are required to study the feedback of various cytoplasmic degradation factors. Although inhibited, the degradation factors might show differential RNA binding behavior during stress response (Jona et al., 2000). The activity of Dcp2 is particularly interesting to analyze after stress induction as it showed the strongest binding to unstable transcripts in our study (Figure 13).

4.2.9 Conservation of general RNA degradation factors in human cells

Many RNA degradation proteins like the decapping and deadenylation factors, the TRAMP complex, the core exosome complex and associated factors are conserved amongst eukaryotes (Houseley and Tollervey, 2009). However, the RNA degradation mechanism in human is far less understood than in yeast. In order to compare different eukaryotic RNA degradation 
pathways, it would be useful to perform PAR-CLIP of key players in RNA decay like the $5^{\prime} \rightarrow 3^{\prime}$ exonuclease Xrn1, the exosome catalytic subunits Rrp6 and Rrp44, as well as the NMD factor Upf1 in human cells. This would allow for a similar analysis as in yeast, regarding binding preference of specific RNA classes, preferred binding sites on mRNAs and antisense transcripts using metagene profiles. Additionally, the comparison with other published RNA binding profiles of human protein complexes could be helpful to further understand the different RNA decay pathways in human cells. In human cells, many more factors are involved in RNA degradation, which makes the understanding and experimental set-up to study RNA decay much more difficult when compared to yeast. In addition, some factors with homologous function have not even been discovered in human cells. For example the factors, which harbor homologues activities like Nrd1-Nab3 for nuclear surveillance in yeast, are assumed for human, but have not been uncovered yet, despite much effort (Arigo et al., 2006; Thiebaut et al., 2006). Systematic analysis of RNA binding factors and their mapping on transcripts might help to identify proteins with Nrd1-Nab3 homologous activities by studying the binding profiles on antisense transcripts. Improved methods such as enhanced crosslinking and immunoprecipitation (eCLIP) may provide a suitable set-up for large-scale and robust profiling of RNA binding proteins (Van Nostrand et al., 2016). 
Transcriptome maps of general eukaryotic RNA degradation factors

- 84 - 


\section{Identification and functional characterization of the novel mRNA modification $N^{\beta}$-methylcytidine (m3C)}

5.1 Results

\subsubsection{Experimental set-up for Trm140 PAR-CLIP and m3C-CLIP}

A

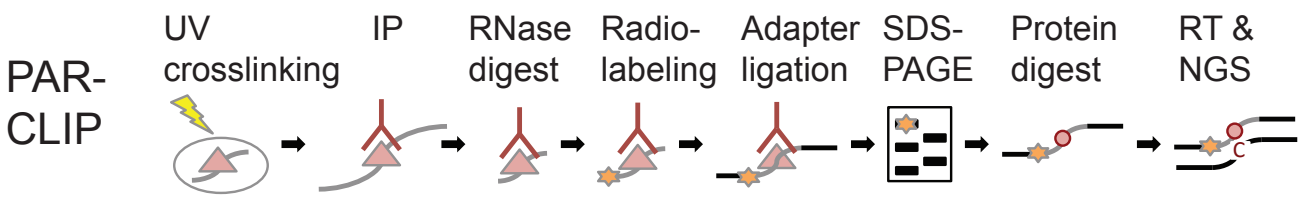

B

m3C-

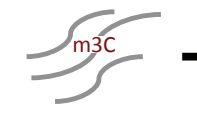

RNA purification

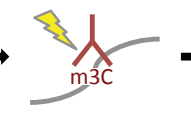

IP \& crosslinking digest

digest

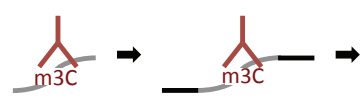

Adapter ligation

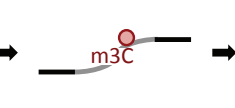

Protein digest

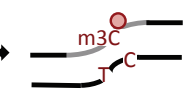

RT \&

NGS

$\mathrm{POI}$

入 Antibody $\approx 32 \mathrm{P}-5$ ' or 3' adapter

- Crosslink site

Figure 14: Schematic overview of PAR-CLIP and m3C-CLIP protocol.

A) PAR-CLIP was performed using $S$. cerevisiae or human cells cultured in the presence of $4 \mathrm{tU}$ or $4 \mathrm{sU}$, respectively. The proteins were cross-linked to RNA by UV light at a wavelength of $365 \mathrm{~nm}$. After cell lysis, the protein of interest was immunoprecipitated with antibody-coupled beads. The crosslinked RNA was fragmented and labeled radioactively using ${ }^{32}$ P-ATP. Adapter ligation was performed on the beads. The protein bound to RNA was purified via SDS-PAGE and the proteinRNA complex corresponding to the predicted size was electro-eluted from the gel. The protein was digested and RNA was converted into cDNA for NGS. B) The protocol for m3C-CLIP was performed with purified and 4tU/4sU-labelled RNA from yeast or human cells. The antibody used for immunoprecipitation against the $\mathrm{m} 3 \mathrm{C}$ modification was crosslinked to the RNA by UV light at the wavelength of $365 \mathrm{~nm}$. The RNA not protected by the antibody was digested and sequencing adapter was ligated to the RNA on the beads. The antibody was digested and recovered RNA was subjected to NGS library generation.

I performed PAR-CLIP of Trm140 and m3C-CLIP as depicted in Figure 14 in the yeast $S$. cerevisiae. The PAR-CLIP experiment was performed as previously published with small adjustments (Baejen et al., 2017, 2014; Battaglia et al., 2017; Creamer et al., 2011). I have adapted the published protocol of PA-m6A-seq to perform m3C-CLIP (Chen et al., 2015). PAR-CLIP and $\mathrm{m} 3 \mathrm{C}$-CLIP protocols overlap in the following steps: by having the medium 
supplemented with 4-thiouracil (4tU), UV crosslinking at $365 \mathrm{~nm}$, and the same library preparation and sequencing strategy. Differences in the mentioned techniques are that immunoprecipitation (IP) was performed against the TAPtag of a protein crosslinked to RNA in the PAR-CLIP experiment compared to m3C-CLIP, where previously purified RNA was directly immunoprecipitated with an anti-m3C antibody and afterwards the antibody-RNA complex was UVcrosslinked. For PAR-CLIP, I performed an additional radioactive RNA labeling and purification step via SDS-PAGE as visualized in Figure 15, which is not needed for the m3C-CLIP protocol. The radioactive signal of the RNA bound to the Trm140 protein appears at the predicted size as verified by Western Blot. Additional radioactive signals were observed for the antibody heavy and light chains. The gel fragment $(1 \mathrm{~cm} \times 0.5 \mathrm{~cm})$ with the highest radioactive intensity including the RNA bound to Trm140 was used for further experimental procedure.

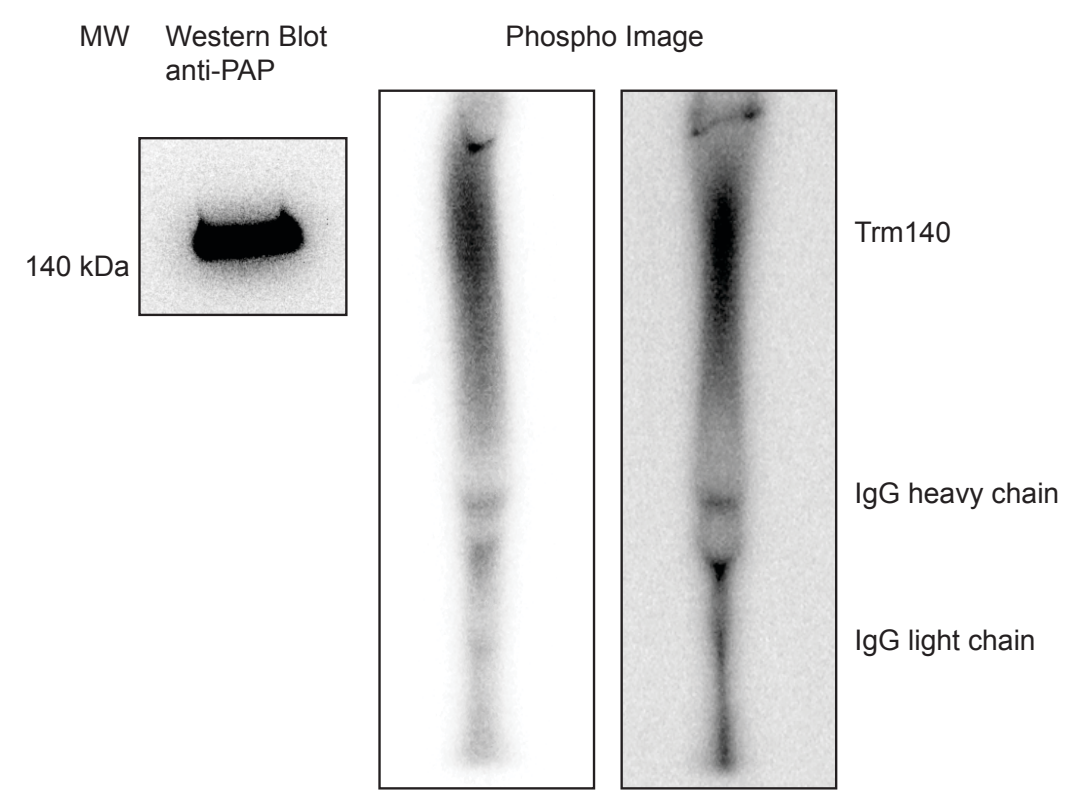

Figure 15: PAR-CLIP of Trm140 analyzed by Western Blot and phosphor imaging.

PAR-CLIP of Trm140-TAP was verified by Western Blot as a size marker. SDS-PAGE was performed with NuPAGE 4-12\% Bis-Tris gels in MOPS buffer. The size separated proteins were transferred onto a PVDF membrane. The protein of interest was visualized using anti-TAP (PAP) antibody coupled to HRP and chemiluminescence reaction. The radioactively labeled RNA bound to the protein of interest was visualized by phosphor imaging after gel electrophoresis (shown for two independent biological replicates). 
5.1.2 Sequencing coverage of Trm140 PAR-CLIP and m3C-CLIP at established $\mathrm{m} 3 \mathrm{C}$ modification sites

In order to cross validate the Trm140 PAR-CLIP and m3C-CLIP experiments, I compared the sequencing read coverage at the established modification site in tRNA Serine and Threonine by using the integrated genomics viewer (IGV) tool (Broad Institute) in Figure 16. I detected typical UV cross-linking induced thymine $(T)$ to cytosine $(C)$ transitions in the variable stem loop for Serine and in the TUC-loop for both analyzed tRNAs in our experiments. The $T \rightarrow C$ transition is shown in the IGV tool as blue bar for $C$ compared to the encoded $\mathrm{T}$ (red) in the reverence genome. The height of the colored bar represents the ratio of the mismatch compared to the encoded base. The UV induced cross-linking sites observed in the Trm140 PAR-CLIP experiment indicate binding of Trm140 to both tRNAs. The UV induced crosslinking sites observed in the $\mathrm{m} 3 \mathrm{C}$-CLIP experiment are caused by $\mathrm{m} 3 \mathrm{C}$ antibody binding to the tRNAs.

Interestingly, at position 32 (black line), where tRNA Serine and Threonine carry the $\mathrm{m} 3 \mathrm{C}$ modification, a high $\mathrm{C} \rightarrow \mathrm{T}$ conversion of $4 \%$ and $24 \%$ was observed for tRNA Serine and $46 \%$ and $36 \%$ for tRNA Threonine in Trm140 PAR-CLIP and m3C-CLIP experiments, respectively. The m3C modification either induces $C \rightarrow T$ mutational signature or reverse transcription (RT) block as previously published (Arimbasseri et al., 2015; D'Silva et al., 2011). These observations showed enrichment of $\mathrm{m} 3 \mathrm{C}$ containing transcripts by Trm140 PAR-CLIP and ability of the $\mathrm{m} 3 \mathrm{C}$ antibody to immunoprecipitate transcripts harboring the $\mathrm{m} 3 \mathrm{C}$ modification. 

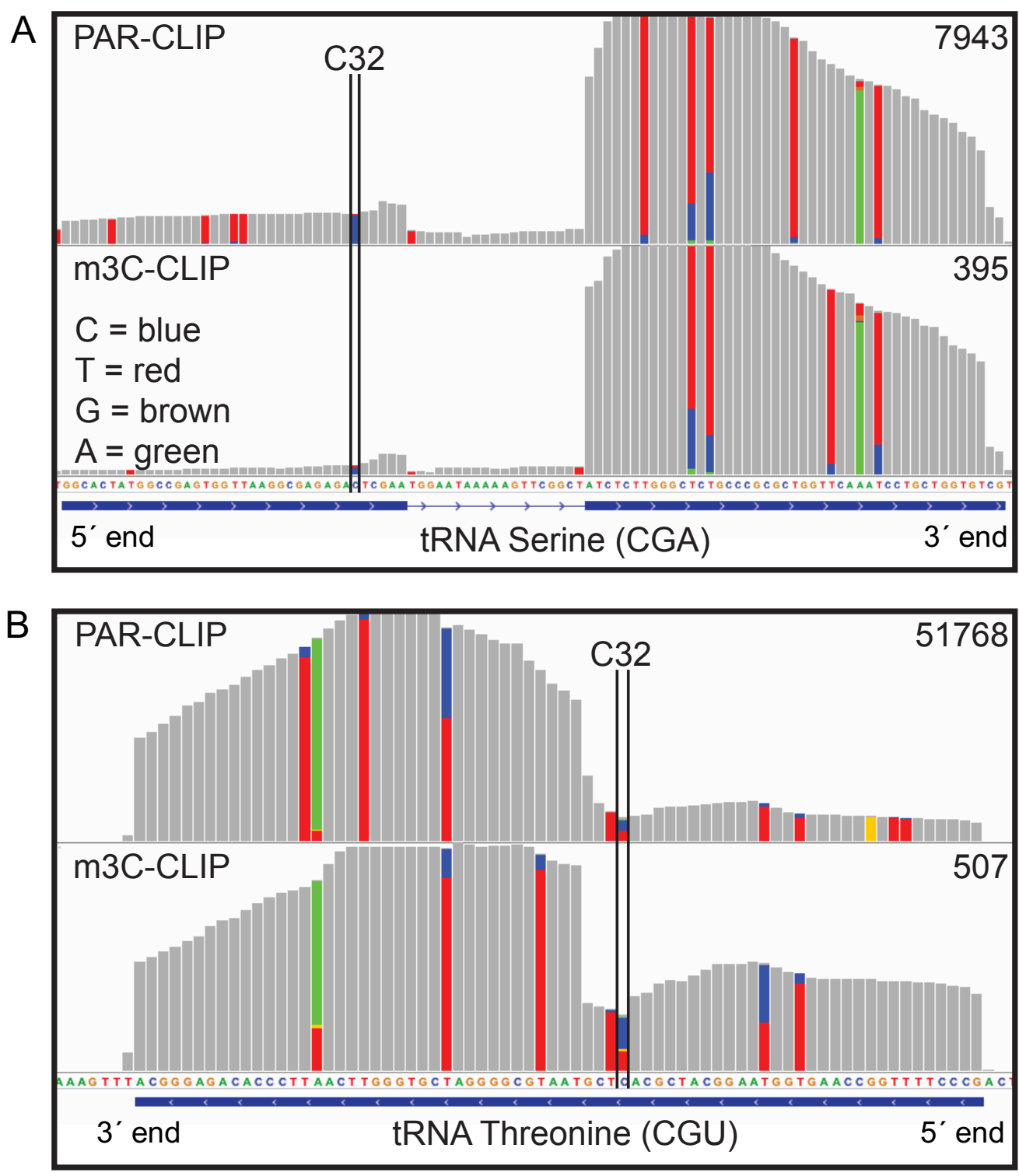

Figure 16: Identification of mutational signature of $N^{3}$-methylcytidine (m3C) modification.

A) Comparison of Trm140 PAR-CLIP and m3C-CLIP sequencing reads visualized using the IGV tool (Broad Institute) on position C32 of tRNA Serine (CGA) known to be $\mathrm{m} 3 \mathrm{C}$ modified by Trm140. C(blue) $\rightarrow \mathrm{T}$ (red) conversion at position 32 indicates $\mathrm{m} 3 \mathrm{C}$ presence. Additional conversions $(T \rightarrow C)$ show PAR-CLIP typical UV induced crosslinking transitions. The DNA coding for the tRNA is located on the Watson strand and highlighted in blue, with the $5^{\prime}$ end at the left and the $3^{\prime}$ end on the right. The $\mathrm{A}$ (green) $\rightarrow T$ transition is caused by $\mathrm{m} 1 \mathrm{~A}$ modification on the respective position shown before (Arimbasseri et al., 2015) B) Trm140 PAR-CLIP and m3C sequencing reads over tRNA Threonine (CGU) (located on the Crick strand: $5^{\prime}$ end on the right, $3^{\prime}$ end on the left) show $\mathrm{C} \rightarrow \mathrm{T}$ conversion induced by $\mathrm{m} 3 \mathrm{C}$ on $\mathrm{C} 32$. The $\mathrm{T} \rightarrow \mathrm{C}$ mutational signatures are caused by UV induced cross-linking. tRNA Threonine also harbors the m1A modification at A58 represented by the typical $A \rightarrow T$ transition (Arimbasseri et al., 2016). 


\subsubsection{Reverse transcription signature of $\mathrm{m} 3 \mathrm{C}$ modification}

The m3C mutational signature has been shown previously as a $C \rightarrow T$ transition or reverse transcription (RT) block (Arimbasseri et al., 2015; D'Silva et al., 2011). An RT block can be explained as a drop of the reverse transcriptase activity during cDNA synthesis due to a bulky RNA structure or the presence of a modified nucleoside. The drop of read coverage next to the modification site in our sequencing data for Trm140 PAR-CLIP and m3C-CLIP (Figure 16) also suggests RT block at the site of the m3C modification. In order to confirm that the RT block is caused by the m3C modification, I performed primer extension assay using a gene specific FAM-labeled primer for tRNA Serine (CGA) and different RNA samples. The products were visualized by denaturing PAGE and fluorescent imaging (Figure 17A). An RT block due to m3C modification results in a shorter fragment, which was observed with $S$. cerevisiae WT RNA. The shorter fragment was absent in the sample using RNA from a Trm $140 \mathrm{KO}$ strain lacking the $\mathrm{m} 3 \mathrm{C}$ modification. The same RT products as with the Trm140 KO RNA were observed for the in vitro demethylated WT sample by AlkB. The AlkB enzyme is a demethylase specific for m1A and $\mathrm{m} 3 \mathrm{C}$ modification derived and purified from E. coli (Aas et al., 2003). The shorter fragment caused by $\mathrm{m} 3 \mathrm{C}$ RT block was again present in the in vitro AlkB demethylated and subsequently in vitro Trm140 re-methylated sample. As a negative control, no RT product was present in the primer alone condition.

The alignment of the RT primer on the sequence and structure of tRNA Serine (CGA) is shown in Figure 17B. The RT block can be explained by the additional methyl group on N-3 of the cytidine as shown in Figure 6 interfering with Watson-Crick base pairing. Additionally, I observed an RT block upstream of the m3C modification site (Figure 17A). This second RT block is most likely due to the N-2-N-2-dimethylguanosine $\left(\mathrm{m}^{2}{ }_{2} \mathrm{G}\right.$ ) modification at G26 (Arimbasseri et al., 2016; Chan and Lowe, 2009). The full-length cDNA product is present above the $\mathrm{m}^{2}{ }_{2} \mathrm{G}$ RT block for all conditions, except the negative control. 
A

B

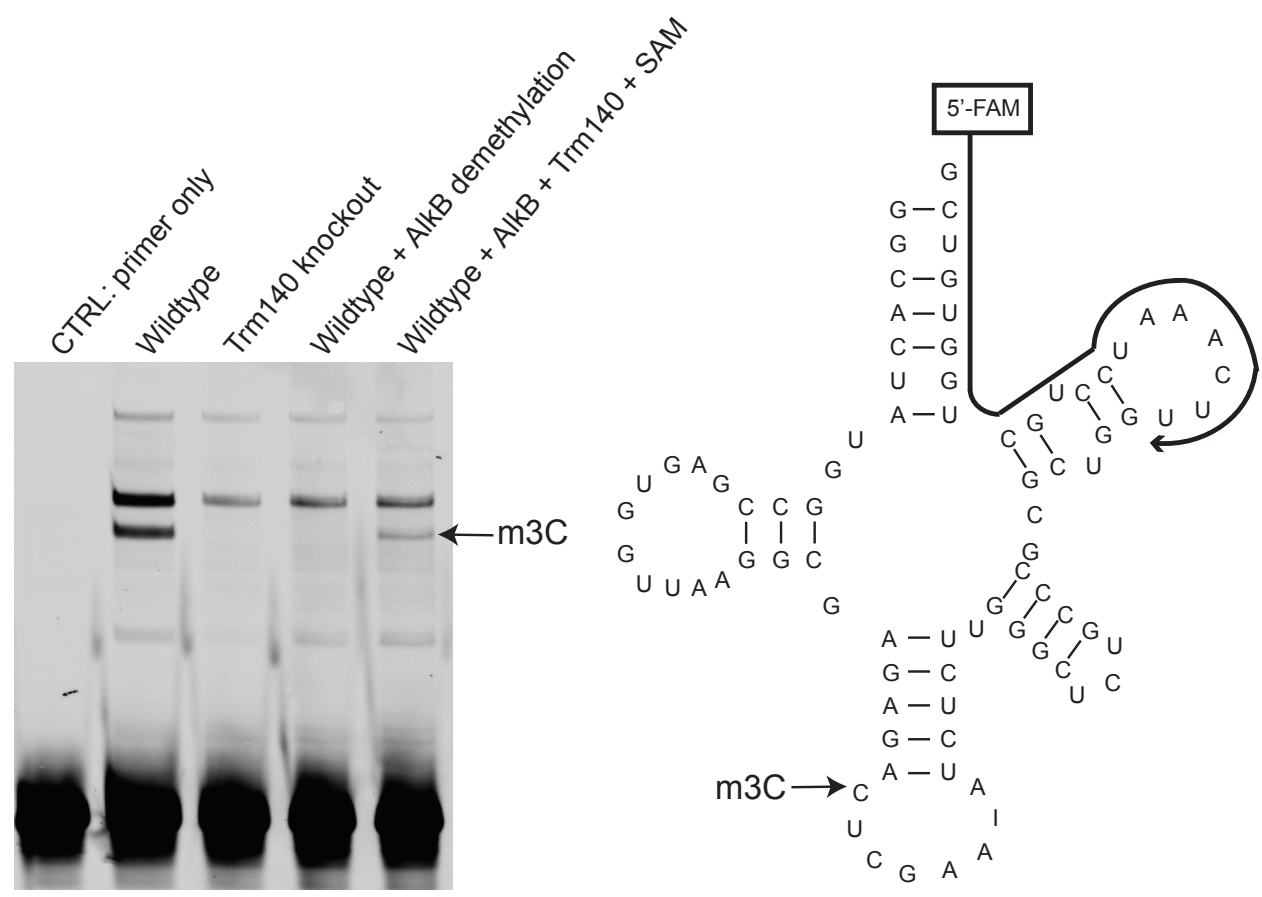

Figure 17: I3C modification causes reverse transcription block shown by primer extension assay on $t R N A$ Serine.

A) Reverse transcription assay was performed with a FAM labeled RT primer complementary to the tRNA Serine (CGA) with different templates: without RNA as negative control (CTRL), WT RNA, Trm140 KO RNA, demethylated and re-methylated RNA. The RT product for each sample was separated on a denaturing $15 \%$ Urea gel for $1 \mathrm{~h}$ at $300 \mathrm{~V}$. At the expected size, I observed a band in the WT and remethylated sample showing $\mathrm{m} 3 \mathrm{C}$ dependent RT block. The RT block of the established modification site of N-2-N-2 dimethylguanosine $\left(\mathrm{m}^{2}{ }_{2} \mathrm{G}\right.$ ) at G26 (above $\mathrm{m} 3 \mathrm{C}$ band) does not change among tested samples. B) Schematic overview of tRNA Serine and RT primer binding (FAM-labeled) and location of the $\mathrm{m} 3 \mathrm{C}$ modification at position C32 in the anticodon loop close to the anticodon (34-36).

\subsubsection{Identification of $\mathrm{m} 3 \mathrm{C}$ modification in tRNA Arginine (CCU)}

In addition to the established modification sites, I observed many UV induced cross-link transitions on all three uridine residues in the TUC-loop of tRNA Arginine with anticodon CCU in the Trm140 PAR-CLIP experiment (Figure 18). This was also shown on established $\mathrm{m} 3 \mathrm{C}$ modification sites in tRNA Serine and Threonine. A high number of UV induced cross-link transitions at the same position suggest strong binding of Trm140 to tRNA Arginine. 
Additionally, I observed m3C-CLIP sequencing reads on tRNA Arginine with anticodon $\mathrm{CCU}$ in both wildtype (WT) replicates. Interestingly, C at position 32 had a high transition rate to $\mathrm{T}$ of about $12 \%$ in the Trm140 PAR-CLIP experiment. This mutational signature has been described for the $\mathrm{m} 3 \mathrm{C}$ modification (Arimbasseri et al., 2015). Furthermore, I observed a high $C \rightarrow T$ transition rate in the m3C-CLIP experiment with WT RNA of $20 \%$ and $42 \%$ for the two replicates. The control $\mathrm{m} 3 \mathrm{C}$-CLIP experiments performed in two replicates with RNA from a Trm $140 \mathrm{KO}$ strain showed only $1 \%$ and $3 \%$ of $\mathrm{C} \rightarrow \mathrm{T}$ transition frequencies. This demonstrates that the mutational signature on $\mathrm{C} 32$ of tRNA Arginine with anticodon CCU was significantly reduced in the Trm140 KO condition. In the PAR-CLIP experiments of Trm140 we also observe that the read coverage drops next to the $\mathrm{m} 3 \mathrm{C}$ modification site due to a potential RT block. These findings indicate that tRNA Arginine is bound by Trm140 and therefore $\mathrm{m} 3 \mathrm{C}$ modified on position $\mathrm{C} 32$ in $\mathrm{S}$. cerevisiae, in addition to the established $\mathrm{m} 3 \mathrm{C}$ modified tRNA Arginine in higher eukaryotes (Arimbasseri et al., 2015). In order to verify the m3C modification on tRNA Arginine with anticodon $\mathrm{CCU}$ in vitro, I performed a primer extension assay using a FAMlabeled RT primer for this particular tRNA. Alignment of the RT primer to the structure and sequence of tRNA Arginine is shown in Figure 18C. The RT assay products were subjected to denaturing PAGE and revealed an RT product corresponding to a shorter fragment in the WT sample in Figure 18B, which was absent in the Trm140 KO condition. Furthermore, it was also absent in the in vitro AlkB demethylated sample and present in the in vitro Trm140 remethylated sample (previously demethylated by AlkB). No RT product was present in the primer alone and no RNA control conditions. The fragment caused by RT block due to $\mathrm{m}^{2}{ }_{2} \mathrm{G} 26$ modification did not change among other tested samples. Taken together, these results show that the RT block is most likely due to the presence of the m3C modification at position C32 in tRNA Arginine (CCU). 


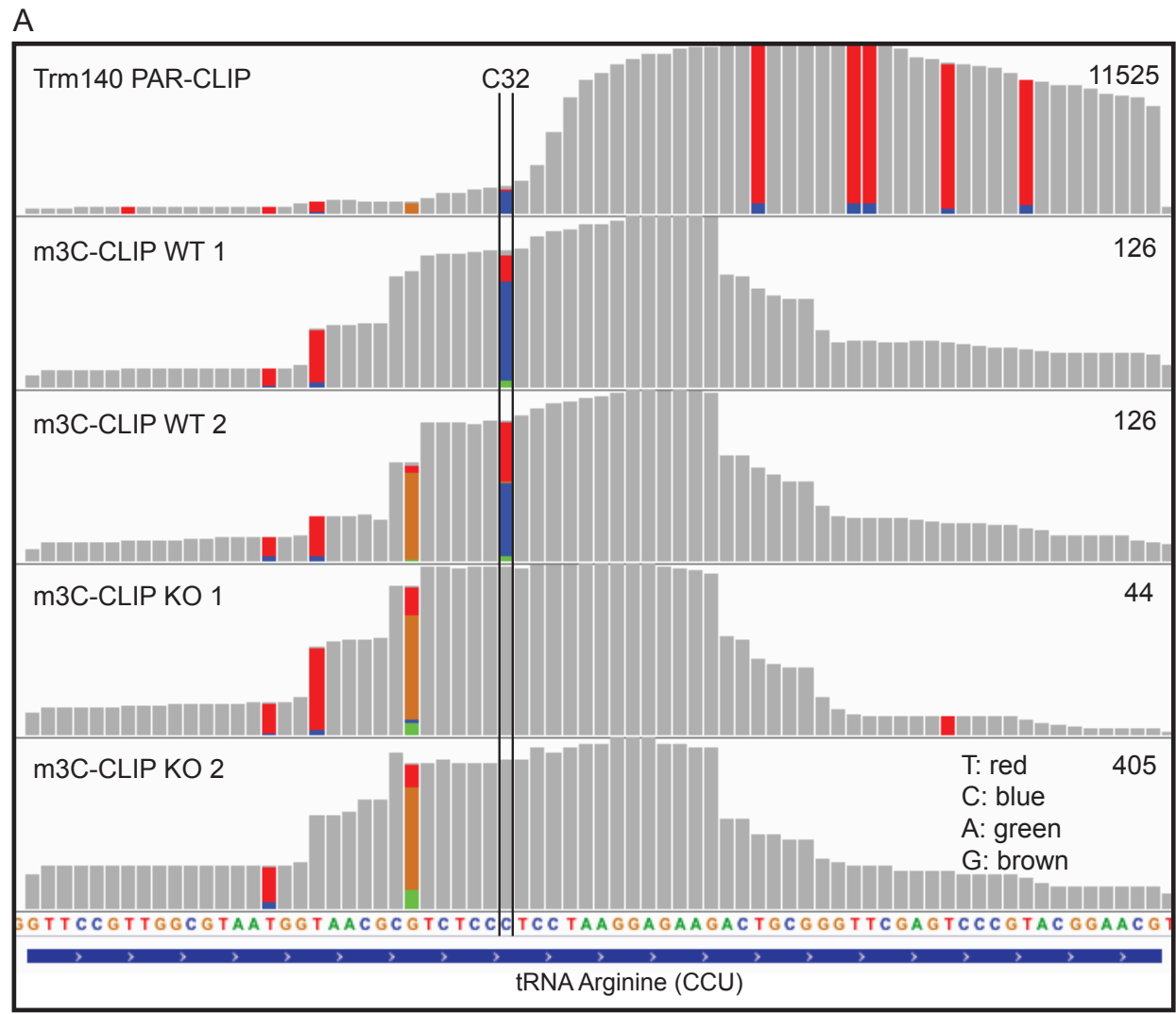

B

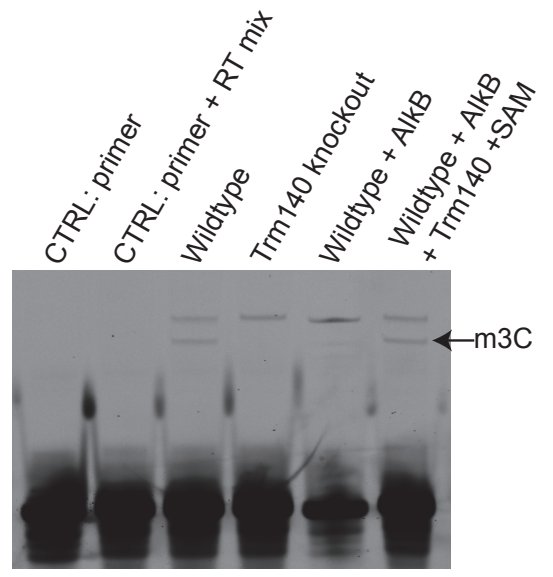

C

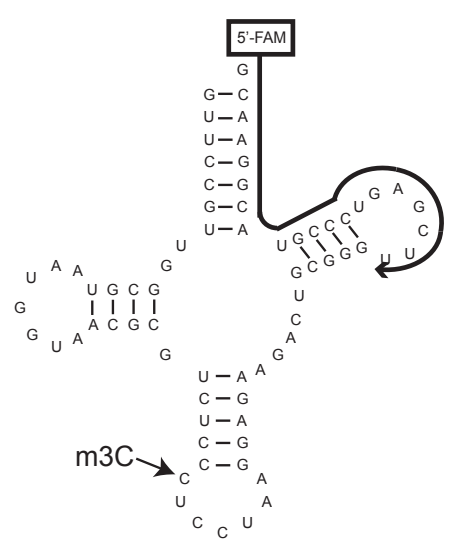

Figure 18: tRNA Arginine (CCU) contains $m 3 C$ at $C 32$ in $S$. cerevisiae.

A) Trm140 PAR-CLIP showed a high $C \rightarrow$ T conversion rate on C32 of tRNA Arginine (CCU). Binding of Trm140 to the T rich TUC-loop of tRNA Arginine is represented as UV-induced $T \rightarrow C$ conversions was visualized using the IGV tool (Broad Institute). The replicates for m3C-CLIP performed with WT RNA showed high $C \rightarrow T$ conversion, whereas the experiment with RNA from the Trm140 KO strain had lower levels of conversion. B) Reverse transcription assay with FAM-labeled primer specific for tRNA Arginine showed a fragment caused by RT block with WT RNA and remethylated RNA, which was absent in Trm140 knockout RNA and demethylated RNA indicating Trm140 dependent $\mathrm{m} 3 \mathrm{C}$ modification on tRNA Arginine. C) Structure and sequence of tRNA Arginine. $\mathrm{m} 3 \mathrm{C}$ modification is indicated with black arrow. FAM-labeled RT primer binding is shown with black arrow starting from the first nucleotide of the $3^{\prime}$ end. 


\subsubsection{Mapping of m3C modification sites in mRNA}

To evaluate Trm40 binding on mRNA and m3C modification enriched sequencing reads in mRNA, we performed metagene analysis on mRNA aligned at the TSS and pA site for Trm140 PAR-CLIP and m3C-CLIP. UV induced cross-linking sites in mRNAs indicate binding of Trm140 within mRNAs and potential m3C sites. Metagene analysis of Trm140 PAR-CLIP and m3CCLIP (Figure 19A) showed binding of the methyltransferase to mRNA and m3CCLIP signal over the protein coding region. Comparing m3C-CLIP to Trm140 PAR-CLIP, we could see binding to the gene body for the first $250 \mathrm{nt}$ of genes and at the end of genes the m3C-CLIP and the Trm140 PAR-CLIP signal is at its maximum.

We analyzed the mutational signatures of $\mathrm{C} \rightarrow \mathrm{T}$ transitions caused by $\mathrm{m} 3 \mathrm{C}$ on a genome wide level. This approach identified potential modification sites within mRNA. A list of high-confidence m3C modification sites is shown in Appendix Table 14. Figure 19B shows an example of $\mathrm{C} \rightarrow \mathrm{T}$ transition at the same position present in Trm140 PAR-CLIP and m3C-CLIP experiments on mRNA Tra1 (genomic position chrVIII:303572). The transition rate was $22 \%$ in PAR-CLIP and 33\% for m3C-CLIP. Another example of high $\mathrm{C} \rightarrow \mathrm{T}$ transition at the same position for both experimental set-ups is shown in Figure 19C for the mRNA Rrs1 (genomic position chrlV:1153577) close to the translation start codon. In this case the conversion rate for Trm140 PAR-CLIP and m3C-CLIP is $69 \%$ and $50 \%$, respectively. In Figure 19D the third example of typical m3C mutational signature is shown on mRNA YCR024C-B (genomic position chrlll:162694) in both experiments close to the stop codon of the transcript. The transition rate is $86 \%$ for the Trm140 PAR-CLIP and $80 \%$ for the $\mathrm{m} 3 \mathrm{C}$-CLIP experiment. In summary, these results indicate the presence of $\mathrm{m} 3 \mathrm{C}$ modification all over the protein coding transcripts. However, metagene analysis of Trm140 PAR-CLIP and m3C-CLIP suggest higher occupancy of the m3C modification at the end of mRNA transcripts. 
A

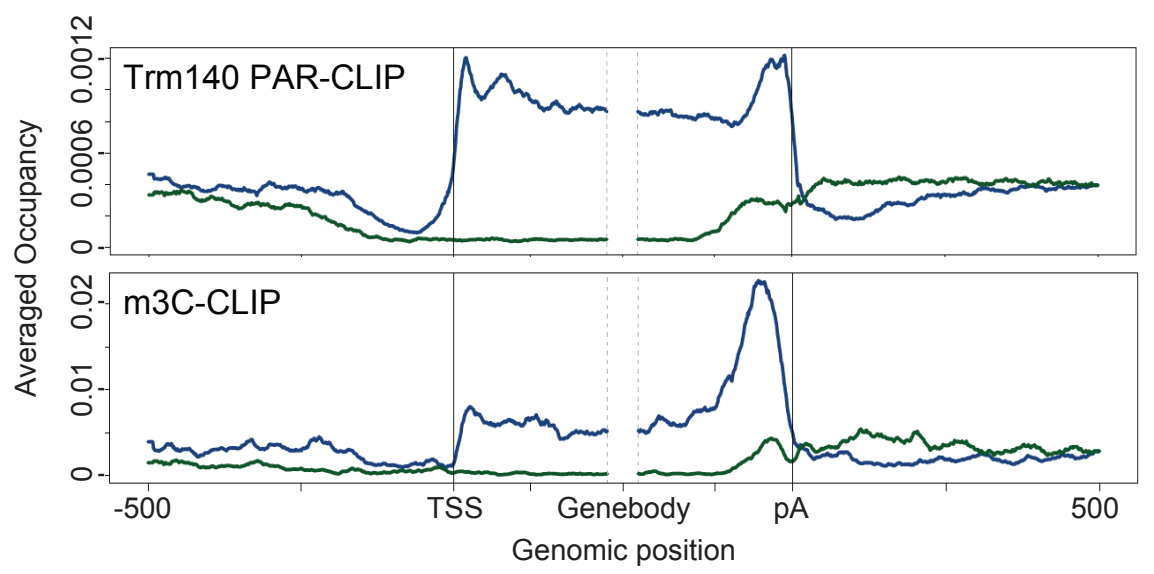

$\mathrm{B}$

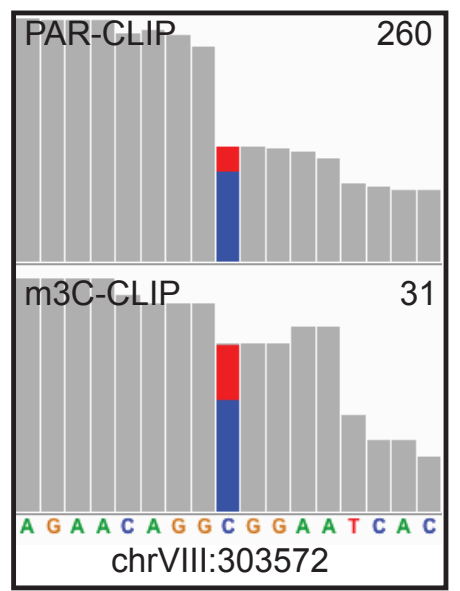

$\mathrm{C}$

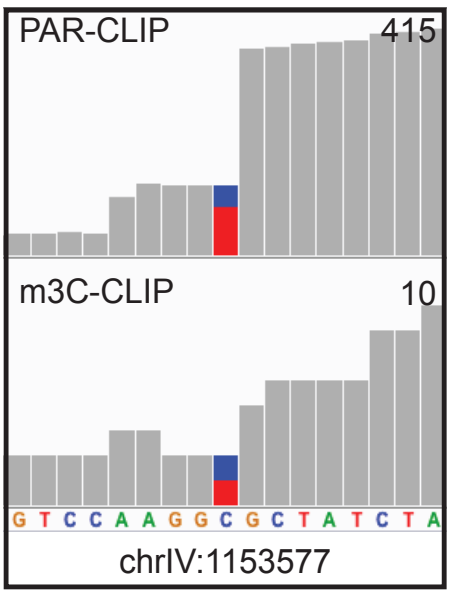

$D$

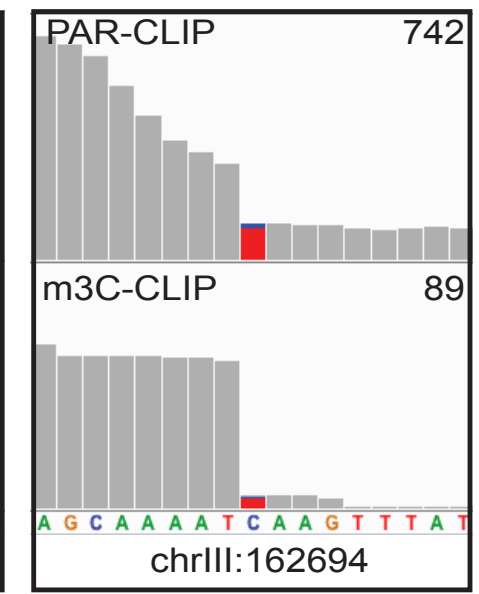

Figure 19: Presence of $N^{3}$-methylcytidine modification in $m R N A$

A) Metagene analysis of Trm140 PAR-CLIP and m3C-CLIP on mRNAs. PAR-CLIP signal over protein coding transcripts with peaks close to TSS and pA site. m3C-CLIP showed signal over the protein coding genes with its maximum at $\sim 75$ bp upstream of the pA site similar to the Trm140 PAR-CLIP. Mutational signature of m3C of Trm140 PAR-CLIP and m3C-CLIP in mRNAs at position chrVIII:303572 in B), chrIV:1153577 in C) and chrlll:162694 in D) visualized using the IGV tool (Broad Institute).

\subsubsection{Translation efficiency change upon m3C depletion}

Chemical modifications on tRNAs and mRNAs, as well as some reader proteins of RNA modifications have been shown to affect translation (Arango et al., 2018; Coots et al., 2017; Li et al., 2017; Nedialkova and Leidel, 2015). In order to test an effect of the $\mathrm{m} 3 \mathrm{C}$ modification on translation via codon occupancy, we used published ribosome profiling data of WT and Trm140 KO cells (Chou et al., 2017). This revealed a change in codon occupancy on tRNA 
Serine, Threonine and Arginine after Trm140 KO. The P-site of the ribosome shows higher occupancy compared to the WT strain on the mentioned tRNAs (Figure 20). This indicates a slower movement of the tRNA from $P$ to $E$ site within the ribosome and therefore slower translation on those codons in general. This suggests an effect on translation due to the absence of the $\mathrm{m} 3 \mathrm{C}$ modification on tRNAs Serine, Threonine, and Arginine.

In addition to the effect of m3C modification on tRNAs, I analyzed the effect on mRNAs in response to the depletion of Trm140. For this, I used the translational efficiency, which is calculated as the ratio between translation (derived from counts of footprints per mRNA in ribosome profiling experiments) over transcription (derived from RNA-seq mRNA levels) of a particular mRNA (Chou et al., 2017). I calculated the translational efficiency for six m3C modified mRNA transcripts on the $\mathrm{m} 3 \mathrm{C}$ containing codons of two independent biological replicates for Trm140 KO and WT cells (Figure 20B). For this, I used the ratio of read counts of ribosome footprints per codon and counts of RNA per codon (Chou et al., 2017). I selected six candidate transcripts based on their m3C modification status showing $\mathrm{C} \rightarrow \mathrm{T}$ transition in Trm140 PAR-CLIP and m3CCLIP experiments (Methods Section 3.3.12). The translational efficiency at the selected codons is increased in the case of Trm140 KO compared to WT condition for all six tested transcripts. This suggests more efficient translation of the respective mRNA codon in the absence of the $\mathrm{m} 3 \mathrm{C}$ modification. This leads to the hypothesis that the m3C modification in mRNA can a roadblock for the ribosome and stalls translation thereby decreasing the translational efficiency. 
A

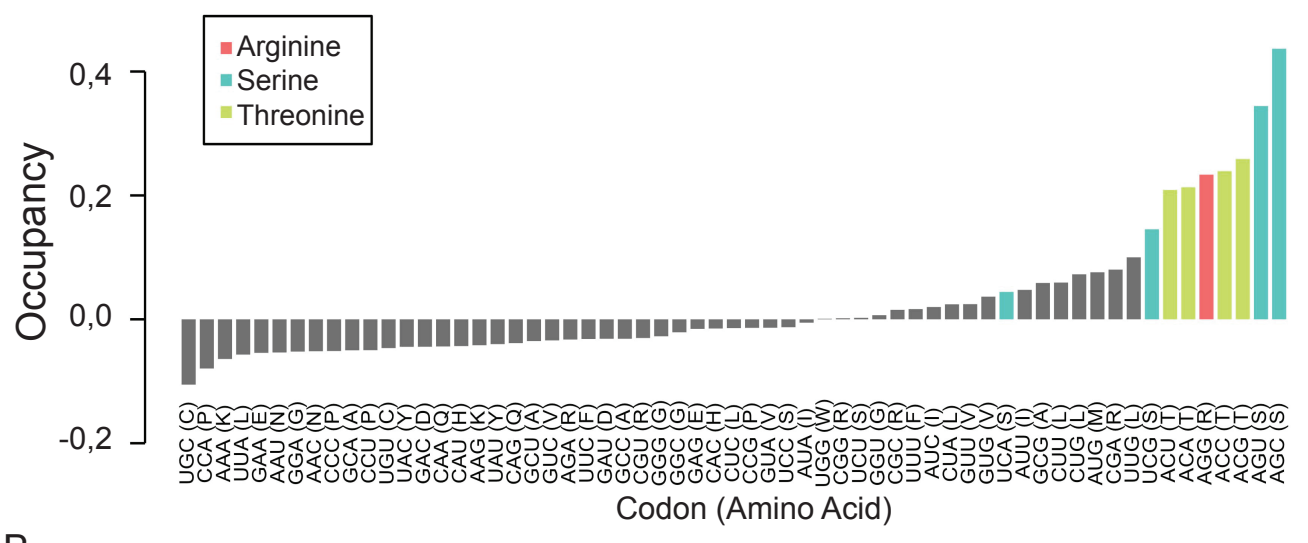

B
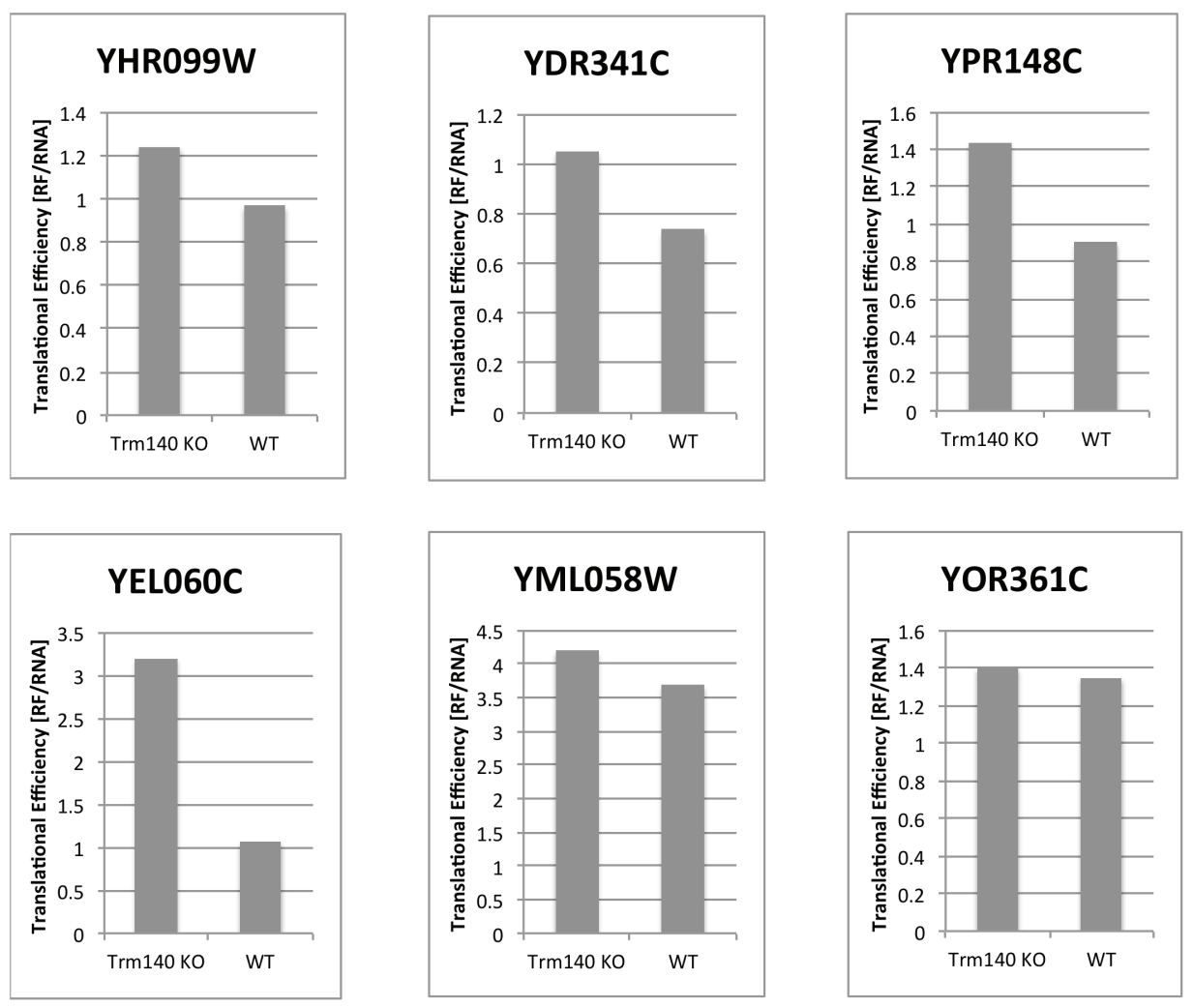

Figure 20: m3C modification loss changes ribosome dynamics.

A) Ribosome profiling of Trm140 KO compared to WT strain showed increased P-site occupancy for the established $\mathrm{m} 3 \mathrm{C}$ containing tRNAs. This indicates prolonged residing time of the respective tRNAs inside the ribosome due to missing $\mathrm{m} 3 \mathrm{C}$ modification on the tRNAs. B) Translational efficiency changes of m3C containing codons shown for six different mRNA transcripts. Translational efficiency is increased in Trm140 KO cells compared to the WT cells for the m3C containing codon. 


\subsubsection{Co-localization of Trm140 with RNA degradation factors}

To evaluate a function of the m3C mRNA and tRNA modification in various processes, we compared Trm140 PAR-CLIP with experiments from general RNA processing and degradation factors comprising nuclear and cytoplasmic processes. Briefly, these factors function in nuclear RNA surveillance (Nrd1, Nab3), 5' cap binding (Cbc2), mRNA transcript elongation (Bur1, Bur2, Ctk1, Ctk2, Cdc73, Ctr9, Leo1, Paf1, Rtf1, Set1, Set2, Dot1, Spt5, Spt6, Rpb1), pre-mRNA splicing (Ist3, Nam8, Mud1, Snp1, Luc7, Mud2, Msl5), pre-mRNA 3' processing (Pab1, Pub1, Rna15, Mpe1, Cft2; Yth1), transcription termination (Rat1, Rai1, Rtt103, Pcf11), mRNA export (Hrp1, Tho2, Gbp2, Hrb1, Mex67, Sub2, Yra1, Nab2, Npl3) (Baejen et al., 2017, 2014; Battaglia et al., 2017; Schulz et al., 2013), deadenylation (Ccr4, Pop2, Not1, Caf40), decapping (Dcp1, Dcp2, Edc2, Edc3, Dhh1), 5’ $\rightarrow 3^{\prime}$ exonuclease (Xrn1) exosome (Rrp6, Rrp40, Csl4, Rrp4, Rrp44), TRAMP complex (Trf4/5, Air1/2, Mtr4), Ski complex (Ski2, Ski3, Ski7, Ski8), and NMD (Upf1, Upf2, Upf3, $\mathrm{Nmd4}$ ). Clustered co-localization analysis in a range of $40 \mathrm{nt}$ around the crosslink site of each factor is shown in Appendix Figure 41. We observed colocalization of Trm140 mostly with cytoplasmic RNA degradation factors. Detailed co-localization analysis with degradation factors in Figure 21 revealed binding sites of Trm140 close to cross-link sites of the deadenylation machinery (Ccr4, Pop2, Not1, Caf40, Pan2, and Pan3), the exosome (Rrp6, Csl4, and Rrp40), as well as the exosome associated complexes TRAMP (via Trf5) and Ski (via Ski7) complex. Assuming Trm140 binding leads to deposition of a m3C modification, this observation suggests a cytoplasmic function of the $\mathrm{m} 3 \mathrm{C}$ modification on RNA degradation. 

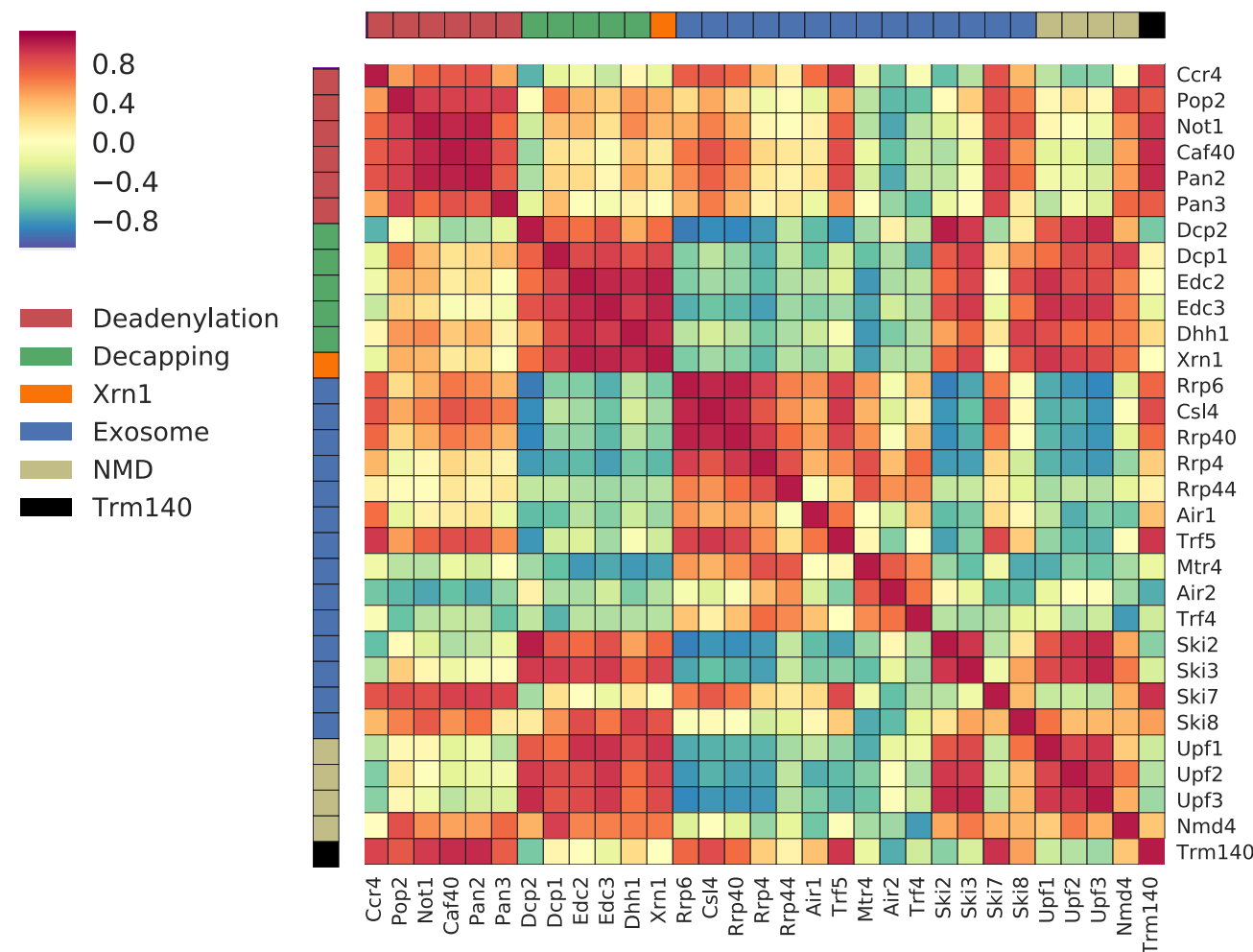

Figure 21: Trm140 co-localizes with various RNA degradation factors.

A) Co-localization analysis $40 \mathrm{nt}$ around the crosslink site of each factor performed with PAR-CLIP data from the deadenylation machinery, decapping factors, $5^{\prime} \rightarrow 3^{\prime}$ exonuclease, exosome and exosome associated complexes TRAMP and Ski as well as NMD compared to Trm140. This revealed high correlation (>0.6) of Trm140 with RNA degradation factors of the deadenylation and exosome machinery, in particular Ccr4, Pop2, Not1, Caf40, Pan2, Pan3, Rrp6, Csl4, Rrp40, Trf5, and Ski7.

5.1.8 Depletion of m3C modification alters RNA synthesis rate and half-life

Metabolic labeling and sequencing of newly synthesized RNA (4tU-seq) can be used to measure RNA synthesis and degradation rates in cells (Schulz et al., 2013; Sun et al., 2012). To study the effect of Trm140 KO and therefore lack of m3C modification on tRNAs and mRNAs, we performed 4tU-seq of Trm140 KO and WT cells. The two biological replicates showed a high correlation for labeled (nascent) and total (steady state) RNA replicates (Figure 22). 
A

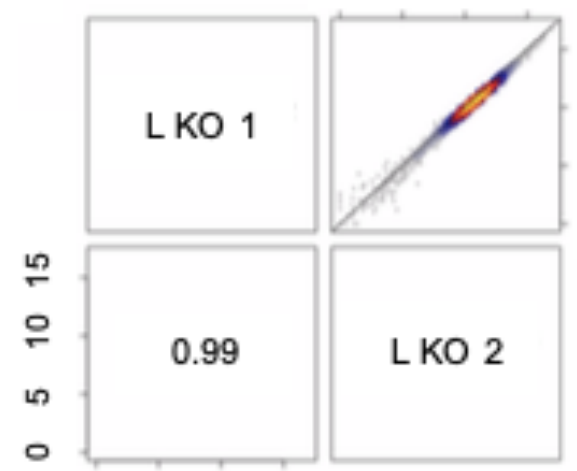

$\begin{array}{llll}0 & 5 & 10 & 15\end{array}$

$\begin{array}{llll}0 & 5 & 10 & 15\end{array}$

C

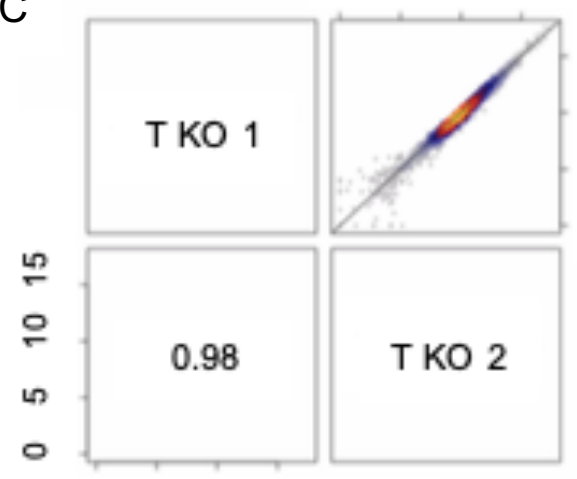

$\begin{array}{llll}0 & 5 & 10 & 15\end{array}$
B

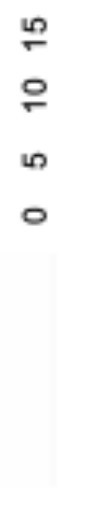

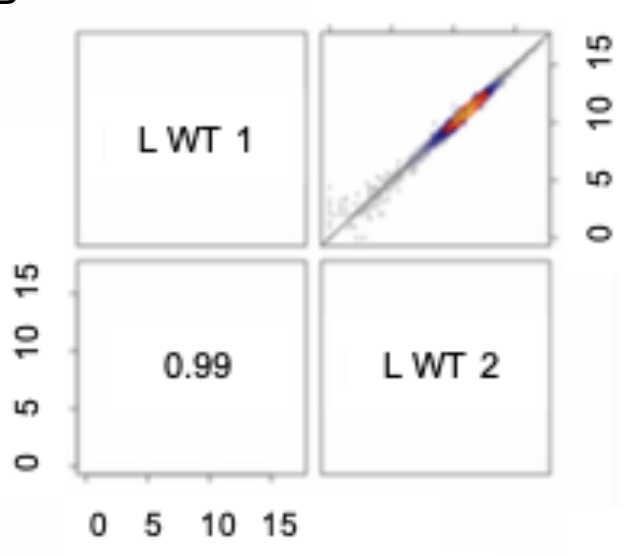

$\begin{array}{llll}0 & 5 & 10 & 15\end{array}$

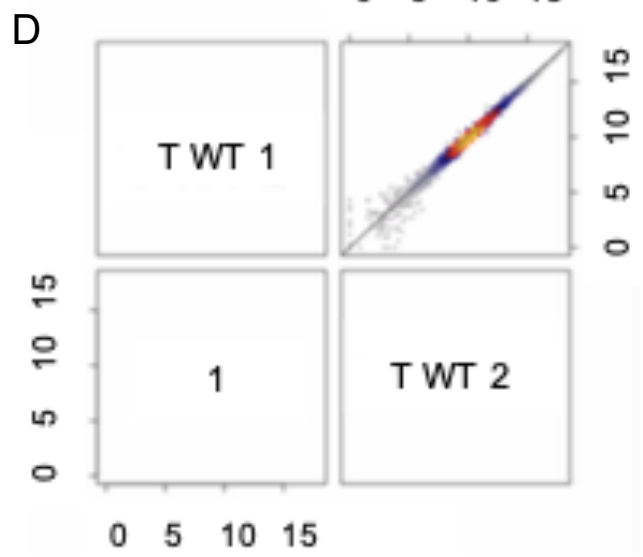

Figure 22: Spearman correlation of $4 t U$-seq data for Trm140 KO and WT cells.

A) The 4tU-seq replicates of Trm140 KO labeled (L) RNA replicate1 and replicate 2 showed a Spearman correlation of 0.99 . B) The replicate correlation of WT L RNA was 0.99. C) The Trm140 KO total (T) RNA replicates showed a correlation of 0.98. D) The correlation of WT T replicates showed a Spearman correlation of 1.

The 4tU-seq datasets (WT and Trm140KO) revealed a globally decreased synthesis rate (Wilcoxon, $p=1.83 \mathrm{e}^{-60}$ ) (Figure 23A) and a slightly increased half-life (Wilcoxon, $p=0.407$ ) of all protein coding transcripts (Figure 23B) in the Trm140 KO condition compared to WT. Overall decreased translational efficiency revealed by ribosome profiling (Chou et al., 2017) in the context of Trm $140 \mathrm{KO}$ cells can result in a reduced protein synthesis. This might explain the reduced RNA synthesis rate due to a feedback loop of less available RNA synthesis machinery. 
A

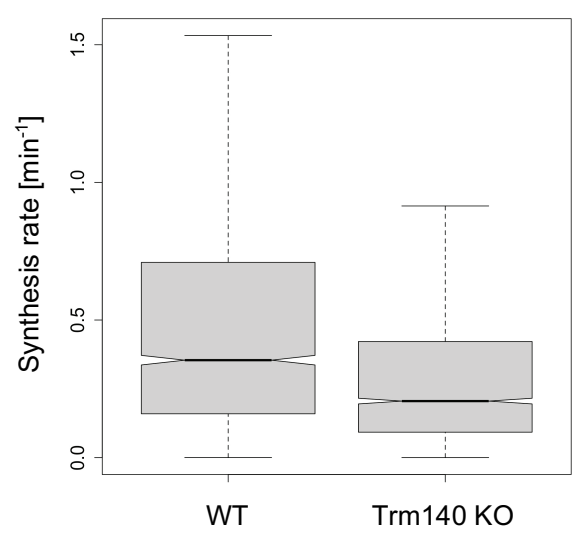

B

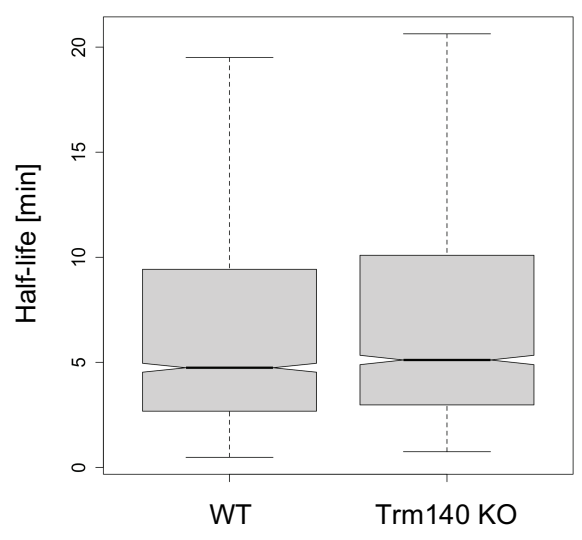

Figure 23: Functional characterization of $m 3 C$ modification loss by using metabolic RNA labeling and sequencing in $S$. cerevisiae.

4tU-seq of WT versus Trm140 KO cells revealed significantly decreased synthesis rate in (in $\mathrm{min}^{-1}$ ) for mRNA in A) and slightly increased half-life (in min) of mRNA in B) in the absence of $\mathrm{m} 3 \mathrm{C}$ modification.

\subsubsection{Experimental set-up for PAR-CLIP of METTL8}

In order to elucidate the localization of the $\mathrm{m} 3 \mathrm{C}$ modification in human cells, I used the inducible Hek293 Flp-In ${ }^{\mathrm{TM}}$ T-REx ${ }^{\mathrm{TM}}$ Cell line (Invitrogen) to generate a C-terminally 3xFLAG tagged METTL8. In this system, protein expression is under the control of a tetracycline inducible promoter. Using this cell line, I performed PAR-CLIP of METTL8 to map its binding sites over the whole transcriptome. For the experiment, different conditions regarding tetracycline induction and crosslinking at the wavelength of $365 \mathrm{~nm}$ in the input and IP samples were tested and examined by Western Blot analysis as shown in Figure 24A. The non-induced sample only showed endogenous level of METTL8 and no signal with the FLAG antibody in the input. Thus, gene expression of METTL8-3xFLAG in the FIp-In ${ }^{\text {TM }}$ T-REX ${ }^{\text {TM }} 293$ cells is only very weak if at all present in the cultured cells under standard conditions. After tetracycline induction, the METTL8 expression level stays constant as shown with the anti-METTL8 antibody and a signal for the FLAG-tagged METTL8 was observed with the anti-FLAG antibody. The Western Blot for the IP samples using the anti-FLAG M2 magnetic beads showed signal only for the tetracycline 
induced samples in both blotting conditions (anti-FLAG and anti-METTL8). The double band in the non-induced IP sample blotted with the anti-FLAG antibody is caused by the heavy chain of the antibody and cross reactivity of the antibodies used for the Western Blot. Radioactive labeling $\left({ }^{32} \mathrm{P}\right)$ of the RNA crosslinked to the enriched protein of interest showed lower signal in the phosphor image for the control samples without tetracycline induction and without crosslinking compared to the two replicates with induction and crosslinking as depicted in Figure 24B. The area of the gel highlighted with black rectangles was used for further experimental procedure.

A

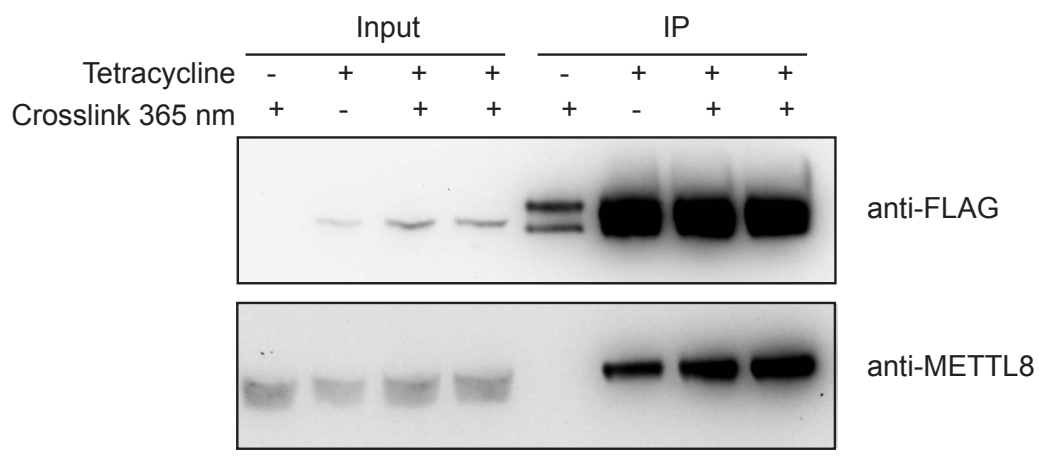

B

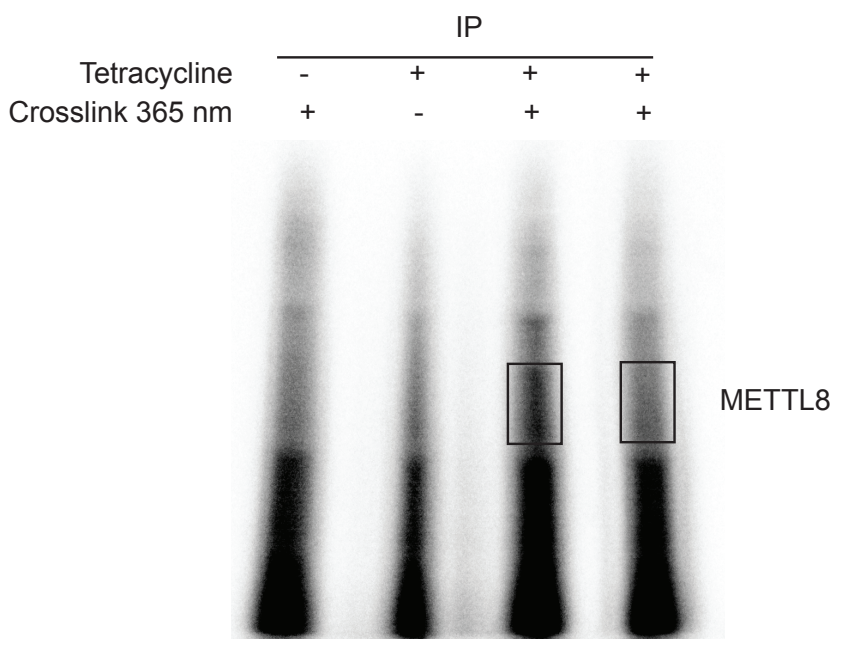

Figure 24: PAR-CLIP of METTL8-3xFLAG in FIp-In TM T-REX ${ }^{\mathrm{TM}} 293$ cells.

A) Western Blot of different conditions varying Tetracycline induction and UVcrosslinking at $365 \mathrm{~nm}$ of Input and IP samples blotted with anti-FLAG and antiMETTL8 antibodies. B) Phosphor image of ${ }^{32} \mathrm{P}$-labeled RNA immuno-precipitated with METTL8-3xFLAG using the same conditions as in the Western Blot. The area cut for further experimental steps is marked on the gel with black rectangles. 
5.1.10 Mapping of the m3C modification in human mRNA

The sequencing data of three independent biological replicates of the 3xFLAG-METTL8 PAR-CLIP experiments were merged for analysis and resulted in 148,781 UV-induced crosslink sites. The UV induced crosslink sites were aligned to protein coding genes at the TSS and pA site and resulted in a metagene plot depicted in Figure 25A. The metagene plot shows binding of METTL8 over the entire length of the mRNAs with higher signal towards the end of transcripts. This indicates a distribution of $\mathrm{m} 3 \mathrm{C}$ modifications over the protein coding transcripts with higher occurrence towards the $3^{\prime}$ end. The search for $\mathrm{m} 3 \mathrm{C}$ mutational signatures $(\mathrm{C} \rightarrow \mathrm{T}$ transition) in the METTL8 PARCLIP experiments resulted in 6144 sites (coverage $>2$, pval >0.005). XXmotif analysis (Luehr et al., 2012) of those sites revealed binding preference of METTL8 to CG rich elements (Figure 25B). The best two hits showed an occurrence of $48.13 \%$ and $20.4 \%$ with E-Values of $3.3 e-24$ and $1.08 e-9$, respectively. 
A

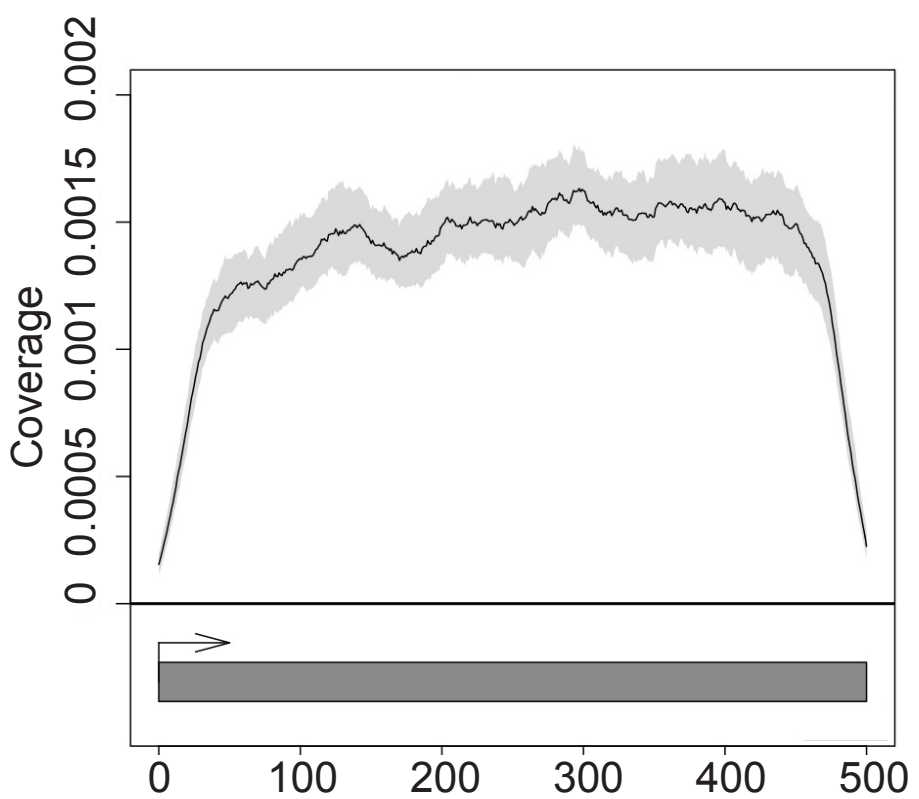

Genomic position of exons scaled and aligned at first TSS of expressed transcription units (bp)

B
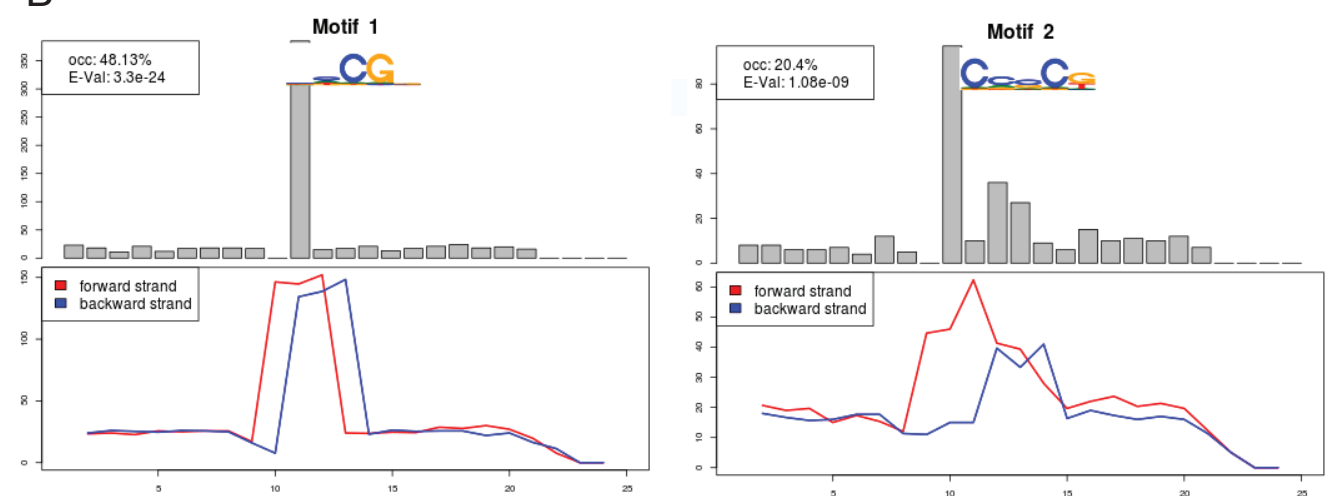

Figure 25: METTL8 PAR-CLIP reveals localization of $m 3 C$ modification in $m R N A$.

A) Metagene analysis of METTL8 PAR-CLIP replicates on exons scaled and aligned at the first annotated TSS. METTL8 cross-link sites are distributed over the entire mRNA. B) XXmotif analysis of the $\mathrm{C} \rightarrow \mathrm{T}$ mutational signature of $\mathrm{m} 3 \mathrm{C}$ modification reveals a CG rich binding element for METTL8. 
5.2 Discussion and Outlook

5.2.1 Identification of the $\mathrm{m} 3 \mathrm{C}$ modification in tRNA Arginine

To study the $\mathrm{m} 3 \mathrm{C}$ modification in the yeast $S$. cerevisiae, I performed PAR-CLIP of the m3C methyltransferase Trm140. The currently established targets of Trm140 are tRNA Serine and Threonine (D'Silva et al., 2011; Noma et al., 2011). I also developed a method called m3C-CLIP, using an anti-m3C antibody for RNA immunoprecipitation analogous to PA-m6A-seq (Chen et al., 2015). I showed that both methods enriched for sequencing reads containing mutational signatures typical for $\mathrm{m} 3 \mathrm{C}$ modification on established targets tRNA Serine and Threonine (Figure 16). I observed that the $\mathrm{m} 3 \mathrm{C}$ modification changes the base paring properties of $\mathrm{C}$ during $\mathrm{RT}$. The mutational signature appears mainly as $\mathrm{C} \rightarrow \mathrm{T}$ conversions as shown in Figure 16 and described before (Arimbasseri et al., 2015). Additionally, the m3C modification induced a RT block close to the modified nucleotide, which has been also observed by others (Arimbasseri et al., 2015; D'Silva et al., 2011). Using these mutational signatures, we were able to map $\mathrm{m} 3 \mathrm{C}$ at single nucleotide resolution in the yeast transcriptome and identify tRNA Arginine with anticodon CCU as a new tRNA target (Figure 18A). The modification was detected at position C32, which is established for tRNA Serine and Threonine (D'Silva et al., 2011; Noma et al., 2011). I also applied a RT assay for tRNA Arginine to confirm m3C presence by RT block (Figure 18B). In order to demonstrate Trm140 dependency of the m3C modification as shown for tRNA Serine and Threonine (D'Silva et al., 2011; Noma et al., 2011), I also demethylated RNA with AlkB (Aas et al., 2003), derived and purified from $E$. coli. The demethylated RNA was subsequently remethylated with purified yeast Trm140, which showed Trm140 methylation dependent RT block in the RT assay (Figure 18B). Using the combined approach of PAR-CLIP, m3C-CLIP and RT assay, I demonstrated the presence of Trm140-dependent m3C modification on tRNA Arginine (CCU) in the anticodon loop at C32. Trm140 has been shown to recognize the sequence G35-U36-t6A37 of the anticodon loop of tRNA Threonine or t6A37 and i6A37 of tRNA Serine (Han et al., 2017). The anticodon loop of tRNA Arginine (CCU) also contains U36 and the i6A37 modification (Chan and Lowe, 2009), which can be the recognition signal for Trm140 to modify this tRNA. 


\subsubsection{Effect of m3C loss on other tRNA modifications}

The m3C-CLIP experiments using RNA from a Trm140 KO strain, as shown in Figure 18A, showed an increased mutational signature for G26 with $G \rightarrow A$ and $G \rightarrow T$ transitions on tRNA Arginine with anticodon CCU using the IGV tool (Broad Institute). This suggest a higher modification level of the nucleotide, which is known to be modified with $\mathrm{m}^{2}{ }_{2} \mathrm{G}$ (Chan and Lowe, 2009). A similar mutational signature was observed before for this modification on G26 of tRNA Serine (Arimbasseri et al., 2016). Higher levels of $\mathrm{m}^{2}{ }_{2} \mathrm{G}$ in the $\operatorname{Trm} 140$ KO strain compared to WT at tRNA Arginine could indicate a compensatory mechanism of the cells in response to the loss of m3C32. The need for a compensatory mechanism could be caused by a destabilized tRNA Arginine structure in the absence of the $\mathrm{m} 3 \mathrm{C}$ modification. In line with this hypothesis, it has been shown before that tRNA modifications at position 32 are important to stabilize the structure of the anticodon loop (Auffinger and Westhof, 2001, 1999). Increased $\mathrm{m}^{2} \mathrm{G}$ modification levels might stabilize the tRNA structure (Steinberg and Cedergren, 1995), in particular the anticodon loop in the context of the Trm $140 \mathrm{KO}$ cells. An increased $\mathrm{m}^{2}{ }_{2} \mathrm{G}$ level has only been observed for tRNA Arginine and not for tRNA Serine and Threonine in our experimental setup. This suggests a more important function of the $\mathrm{m} 3 \mathrm{C}$ modification on tRNA Arginine in the metabolism of the cell. This is also reflected in the $P$ site occupancy analysis of the ribosome (Figure 20), where tRNA Arginine showed a strong increase compared to most other tRNAs.

\subsubsection{Functional relevance of the $\mathrm{m} 3 \mathrm{C}$ modification}

For functional analysis, it has previously been shown in experiments with human cells that the ratio of ribosomes associated in polysomes to monosomes is slightly decreased in METTL8 KO compared to WT conditions (Xu et al., 2017). This suggests increased ribosome stalling when METTL8 and thus the $\mathrm{m} 3 \mathrm{C}$ modification was absent. In addition to that, ribosome profiling analysis in yeast WT and Trm140 KO strains (Chou et al., 2017) showed increased P site ribosome occupancy on those codons, which are complementary to the tRNAs 
lacking the m3C modification in the Trm140 KO strain (Figure 20A). The slower translation rate in absence of the $\mathrm{m} 3 \mathrm{C}$ modification could be explained by incorrect modified tRNAs (Chou et al., 2017). However, our analysis revealed enhanced translational efficiency on m3C harboring codons (Figure 20B). Furthermore, 4tU-seq of the Trm140 KO in comparison to WT strain revealed an impact of the m3C modification on mRNA stability (Figure 23). Our analysis showed a decreased synthesis rate and a slightly increased half-life for mRNAs in the Trm140 KO compared to WT strain (Figure 23).

In the light of existing literature, our findings suggest that there are two contradictory effects: the role of m3C modification in tRNA versus in mRNA. The lack of $\mathrm{m} 3 \mathrm{C}$ modification in tRNAs might destabilize the structure of the anticodon loop (Auffinger and Westhof, 2001, 1999). This may lead to an inefficient accommodation in the active center of the ribosome and therefore ribosome stalling. On the other hand, the $\mathrm{m} 3 \mathrm{C}$ modification in mRNA can hinder Watson Crick base pairing (Figure 26) of the mRNA codon with the tRNA anticodon within the actively translating ribosome. The impaired Watson Crick base pairing is explained by $\mathrm{H}-\mathrm{H}$ repulsion of the $N^{3}$-methyl group of $\mathrm{m} 3 \mathrm{C}$ with the $N^{1}-\mathrm{H}$ proton of $\mathrm{G}$. This impeded Watson Crick base pairing of $\mathrm{m} 3 \mathrm{C}$ with $\mathrm{G}$ has been observed also in our RT assay by RT block (Figure 17A and Figure 18B). The hindered Watson Crick base pairing between $\mathrm{m} 3 \mathrm{C}$ and $\mathrm{G}$ suggests that the $\mathrm{m} 3 \mathrm{C}$ modification in mRNAs stalls the ribosome like a roadblock and can thereby lead to recruitment of degradation factors (Radhakrishnan et al., 2016). Indeed, comparison of Trm140 PAR-CLIP data with genome wide RNA binding data of general processing factors in yeast revealed strong colocalization coefficients of Trm140 binding sites on transcripts with crosslinksite of degradation factors, particularly deadenylation factors (Ccr4, Pop2, Pan2 and Pan3), several exosome components (Rrp6, Cs14, and Rrp40) and an exosome associated factor (Ski7) (Figure 21). Co-localization with general cytoplasmic RNA degradation factors indicates a function of the $\mathrm{m} 3 \mathrm{C}$ modification in mRNAs in the cytoplasm. Localization of Trm140 to the actin cytoskeleton has been shown before, supporting further a cytoplasmic deposition of the m3C modification (Kilchert and Spang, 2011). Our data analysis on translational efficiency suggests ribosome stalling upon translation of codons harboring the m3C modification (Figure 20) and this can lead to 
A

C

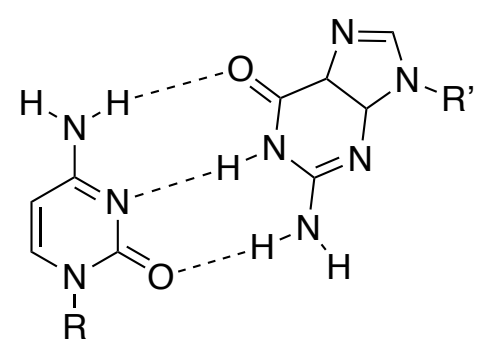

B

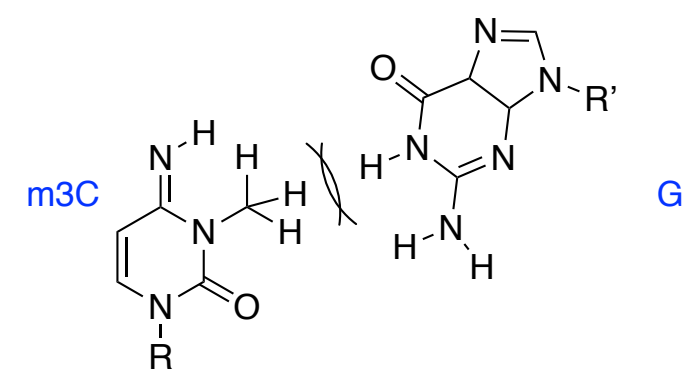

Figure 26: Schematic drawing of Watson Crick base pairing between unmodified $C$ and $G(A)$ as well as $H-H$ repulsion of $m 3 C$ and $G(B)$.

indirect recruitment of general degradation factors in the cytoplasm. This hypothesis is supported by previous findings regarding the response of ribosome stalling on premature termination codons during NMD (Celik et al., 2017). During this process, the ribosome is blocked on premature stop codons and factors that initiate mRNA degradation by endonucleolytic cleavage, deadenylation (by the Ccr4-Not and Pan2/Pan3 deadenylation complexes) and/or decapping (by the decapping complex) are recruited (Lykke-Andersen and Jensen, 2015). It is also known that the Ski complex interacts with stalled ribosomes in case of NMD and links mRNAs to deadenylation and exosomal degradation (Mitchell and Tollervey, 2003). From the work of Pelechano et al., it is known that ribosome stalling increases degradation of the transcript from the $5^{\prime}$ end (Pelechano et al., 2015). This is also in agreement with studies from Radhakrishnan et al. that the degradation machinery is recruited upon ribosome stalling. Ribosome stalling can be monitored by Dhh1, a decapping enhancer, which travels with the ribosome and senses translational efficiency (Radhakrishnan et al., 2016). In our co-localization analysis we observed also medium enrichment of Dhh1 crosslink-sites in proximity to Trm140 binding sites (Figure 21). Thus, our observations regarding co-localization of Trm140 with RNA degradation factors can be placed into context with existing literature mentioned above. Moreover, the lack of the m3C mRNA modification acting as a roadblock or "degradation signal" might explain the slightly prolonged half-life of mRNAs in the Trm140 KO cells as shown in Figure 23. However, the exact 
mechanism how the $\mathrm{m} 3 \mathrm{C}$ modification in $\mathrm{mRNA}$ can stall the ribosome or recruit degradation factors directly needs to be further analyzed.

5.2.4 Future directions for studies on the identification of $\mathrm{m} 3 \mathrm{C}$ binding proteins in different eukaryotic cells

Epigenomic marks such as 5-methylcytosine in DNA can be recognized by specific binding proteins, thereby mediating its repressive effects (Klose and Bird, 2006). In addition to selective DNA modification binding proteins, methylation of RNA at specific positions can also affect interacting proteins (Dominissini et al., 2012). Novel m6A binding proteins were identified using methylated and negative control RNA baits in an RNA affinity chromatography approach accompanied with mass spectrometry analysis (Dominissini et al., 2012). Identification of $m 6 A$ reader proteins was additionally performed by in vitro assays and binding experiments with a recombinantly expressed YTH domain. These studies showed RNA binding of the YTH domain in an m6Adependent manner, although with different binding affinities, some in the nM range (Li et al., 2014; Luo and Tong, 2014; Zhu et al., 2014) and others with $\mu \mathrm{M}$ affinity (Theler et al., 2014; Xu et al., 2015). Identification of different m6A binding proteins revealed additional implications of the m6A modification in various processes during the life-time of an RNA molecule (Han et al., 2019; Kretschmer et al., 2018; Lence et al., 2016; Theler et al., 2014; Wang et al., 2015; Y. Wang et al., 2014; Xiao et al., 2016). Identification of m3C binding proteins is required in the future to study the role of the modification in detail. This may be pursued in a similar way to the identification of $\mathrm{m} 6 \mathrm{~A}$ binding proteins (Dominissini et al., 2012): Using RNA affinity chromatography of m3C modified and control RNA with yeast and human cellular extract in combination with mass spectrometry analysis. These experiments can reveal the $\mathrm{m} 3 \mathrm{C}$ interactome of yeast and human cells. Identification of specific binding proteins may uncover novel functions of the m3C mRNA modification. Identification of potential eraser proteins with homologous function to AlkB from E. coli (Trewick et al., 2002) can additionally reveal a dynamic nature of the $\mathrm{m} 3 \mathrm{C}$ modification. A potential candidate to reverse the $\mathrm{m} 3 \mathrm{C}$ modification in human cells is the 
ALKBH3 protein, which has been shown to repair methylation damage in DNA and RNA (Aas et al., 2003; Duncan et al., 2002; Lee et al., 2005; Zhao et al., 2015).

5.2.5 Functional implication of the $\mathrm{m} 3 \mathrm{C}$ modification in the context of the epitranscriptome

In this dissertation, the localization of the $\mathrm{m} 3 \mathrm{C}$ modification was analyzed by metagene binding profiles on mRNA for the methyltransferases Trm140 and METTL8 in yeast and human cells. The analyses revealed a binding preference for both enzymes over the protein coding sequence with higher occupancy at the pA site (Figure 19A, Figure 25A). A similar localization of the m6A modification (see Figure 5) has been shown before in mouse and human mRNAs (Dominissini et al., 2012). Due to a similar localization of the $\mathrm{m} 6 \mathrm{~A}$ and $\mathrm{m} 3 \mathrm{C}$ modifications, they may have similar functional implications. Epitranscriptomic marks may decorate the RNA and thereby influence cisregulatory elements within the protein coding transcript. Various cis-regulatory elements within the RNA are highly enriched in the 5' and 3' UTRs regulating RNA localization, stability, and translation (Mignone et al., 2002). Reader proteins of the m6A modification are associated with mRNA stability and translation (Theler et al., 2014; Wang et al., 2015; X. Wang et al., 2014; Xiao et al., 2016). According to the analysis of a reduced translational efficiency for m3C harboring codons in yeast (Figure 19B), the m3C modification might also have specific binding proteins to influence ribosome stalling. Our co-localization analysis (Figure 21) revealed several potential binding partners or interacting partners of these complexes not included in the analysis here. In order to get a better understanding of the $\mathrm{m} 3 \mathrm{C}$ modification, its function and to compare it with other epitranscriptomic marks, one needs to identify and study the m3C interactome.

It has been shown for the m6A modification to act as a switch for the mRNA secondary structure to expose cis-regulatory elements and to regulate protein-RNA interactions as shown in Figure 27 (Liu et al., 2015). Due to its ability to hinder Watson Crick base pairing of the $\mathrm{m} 3 \mathrm{C}$ modification with $\mathrm{G}$ 
(Figure 26), this epitranscriptomic mark harbors the potential to change RNA secondary structures. This might in turn allow access for RNA binding proteins to the single stranded RNA analogous to the m6A mark as shown in Figure 27.

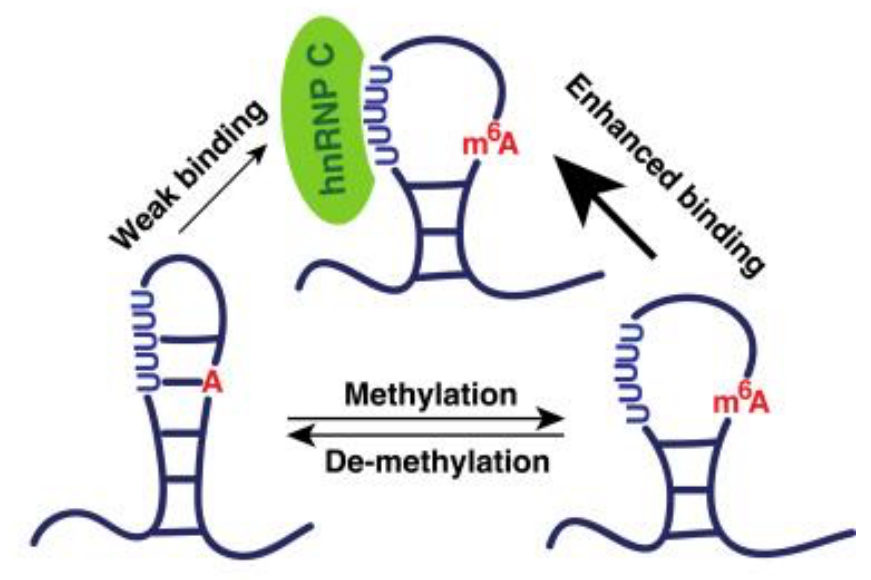

Figure 27: Illustration of the m6A switch model.

Reversible m6A methylation changes the secondary structure of mRNA to regulate RNA-protein interactions (from Liu et al., 2015).

5.2.6 Dissecting functional implications of the $\mathrm{m} 3 \mathrm{C}$ modification in tRNA and mRNA in the future

In the context of Trm140 KO strain, we observed increased $\mathrm{P}$ site occupancy for m3C modified tRNAs (Figure 20A) implying reduced translational efficiency. In contrast, we observed higher translational efficiency in the absence of $\mathrm{m} 3 \mathrm{C}$ on mRNA codons (Figure 20B). Global ribosome profiling (Chou et al., 2017) and 4tU-seq were not able to reveal the contradictory effects of the $\mathrm{m} 3 \mathrm{C}$ modification in tRNAs and mRNAs independently. In order to distinguish the effect of the $\mathrm{m} 3 \mathrm{C}$ modification on the level of tRNA and mRNA separately, different experimental set-ups need to be used.

One idea is to use the Trm140 KO strain but complementing the cells with purified tRNAs from WT strains to ensure proper tRNA modifications. Using this approach, the measured effects by ribosome profiling and 4tU-seq should just be caused by the missing $\mathrm{m} 3 \mathrm{C}$ modification in mRNAs. On the other hand, this approach has various disadvantages. For one, uptake of the tRNAs into 
the cytoplasm cannot be guaranteed. For two, the concentration of tRNA pool would be changed, which would influence the availability of all tRNAs during translation and thereby artificially perturb translational efficiency. It has been shown that yeast cells use differential expression of tRNAs to selectively adjust protein synthesis under stress conditions (Torrent et al., 2018). Inducing a stress situation by altered tRNA availability might additionally perturb gene expression.

An alternative approach could be the application of a fast degradation system for the methyltransferase Trm140. A fast degradation system was developed recently, using the dTAG mechanism (Nabet et al., 2018). This enables the immediate and selective control of single protein abundance by using the novel degrader FKBP12 $2^{\mathrm{F} 36 \mathrm{~V}}$ and in frame expression of FKBP12 ${ }^{\mathrm{F} 36 \mathrm{~V}}$, fused to the protein of interest. This technology provides kinetic resolution to biological analyses such as RNA modifications (Nabet et al., 2018). Rapid degradation of Trm140 would lead to loss of the m3C modification on shortlived mRNAs, while the $\mathrm{m} 3 \mathrm{C}$ modification would in theory still be present on long-lived tRNAs. This may enable us to distinguish the effect of the m3C modification on mRNAs and tRNAs. Changes in translational efficiency and RNA metabolism should now be only caused by the m3C mRNA modification.

5.2.7 Functional characterization of the $\mathrm{m} 3 \mathrm{C}$ modification in human cells in the future

METTL8 PAR-CLIP has revealed the presence of the $\mathrm{m} 3 \mathrm{C}$ modification in mRNAs and its localization within mRNAs (Figure 25A). However, functional studies such as ribosome profiling and RNA-seq are complementarily required to gain a better understanding of the function of the $\mathrm{m} 3 \mathrm{C}$ modification in human cells. To study the effect of the m3C modification on mRNA, depletion of METTL8 is necessary to compare the effect of the $\mathrm{m3C}$ modification loss to wildtype cells. It has been shown that cells having a KO of METTL8 are viable (Xu et al., 2017). However, permanent KO of METTL8 could lead to secondary effects on long-lived RNAs. Depletion of METTL8 in a short regime of time, can help to study the functional implications of the $\mathrm{m} 3 \mathrm{C}$ modification in mRNAs. For 
rapid depletion of METTL8, the application of the dTAG system in human cells is possible as well (Nabet et al., 2018). Subsequent to METTL8 depletion, transient-transcriptome sequencing (TT-seq) similar to $4 \mathrm{tU}$-seq is applicable to study the effect of m3C loss on RNA metabolism (Schwalb et al., 2016). It is now possible to calculate RNA synthesis and degradation rates from the sequencing data analogous to the analysis in yeast (Figure 23, Methods). In addition to the RNA metabolism, the effect of $\mathrm{m} 3 \mathrm{C}$ loss in mRNAs on translation efficiency may be studied by ribosome profiling (Ingolia et al., 2009). Using both methods, TT-seq and ribosome profiling, in combination with the dTAG system, may give detailed insights on potential effects of the $\mathrm{m} 3 \mathrm{C}$ modification in mRNAs of human cells.

5.2.8 Technical challenges in the field of epitranscriptomics and future perspectives

Our understanding of the localization, function and regulation of the currently known dynamic RNA modifications relies on sequencing technologies (Li et al., 2016b). However, RNA modifications cannot be detected directly so far on a high throughput level, since sequencing technologies depend on reverse transcription of RNA into cDNA. This process erases in many cases the information about RNA modifications, which do not change the base pairing properties of the modified bases. In addition, mRNA is of low abundance compared to tRNA and rRNA and this increases the challenge to detect specific base modifications. Thus, new experimental approaches are required to identify novel mRNA modifications and map them with nucleotide resolution. Potential techniques to study mRNA modifications in more detail may involve specific antibodies against the modification of interest or direct RNA sequencing approaches (Arango et al., 2018; Chen et al., 2015; Dominissini et al., 2016, 2012; Liu et al., 2019; A. M. Smith et al., 2017). A very promising technique to study RNA modifications using direct RNA sequencing was developed by Oxford Nanopore Technologies to overcome current limitations of the available techniques (Hussain, 2018; Liu et al., 2019; A. M. Smith et al., 2017). Hereby, a synthetic, electrically resistant membrane is interspersed with large 
nanopores. A voltage is applied across this membrane providing an ionic current across the pores. RNA molecules fused to a tether protein are directed to nanopores and a motor protein pulls individual RNA strands through the biological nanopores at a controlled rate. Each RNA base shows a specific current profile across the nanopores and this allows calling of the nucleotide composition of the RNA sequence. However, this method possesses a sequencing accuracy of only around $85 \%$ (experience from our laboratory and personal communication) compared to $99 \%$ standard Illumina DNA sequencing (Fox et al., 2014). Thus, the accuracy of Oxford Nanopore Technologies direct RNA sequencing does currently not allow to distinguish between mutational signatures caused by an RNA modification or pure sequencing error. With an improved sequencing accuracy, this direct RNA sequencing technique may be able to distinguish modified nucleosides within an RNA stretch. A very recent study proposed that the presence of RNA modifications causes current intensity changes leading to decreased qualities and increased "errors" in the output of base-calling algorithms. Using this model and a machine learning approach, the authors showed that base-calling "errors" mapped m6A modifications in native RNA sequences with an overall accuracy of $~ 90 \%$ (Liu et al., 2019). In order to identify the $\mathrm{m} 3 \mathrm{C}$ modification in native RNAs, the current intensity change due to the modification needs to be determined upon comparison with the raw current intensities.

It is important to note that not only experimental and technical improvements need to be introduced to improve the quality of the identification and calling of modifications, but also data analyses. It has been observed before that next-generation sequencing data has to be handled carefully to not draw false conclusions from it (Schwartz, 2018). Sequencing reads have to be mapped to the reference genome with high accuracy in order to call mutational signatures and reverse transcription blocks caused by modified nucleosides (Schwartz, 2018). Reverse transcription blocks occur only at the end of sequencing reads and thus, soft clipping of their ends during mapping may change the resulting read end and its localization (Schwartz, 2018). In addition, a sufficient sequencing depth is required to be able to call low abundant mRNA modification sites (Xiong et al., 2018). Thus, experimental and technical improvements to overcome current limitations are the most promising approach 
to advance studies in the field of epitranscriptomics in the future (Jantsch and Schaefer, 2019). 


\section{Bibliography}

Aas PA, Otterlei M, Falnes PO, Vagbo CB, Skorpen F, Akbari M, Sundheim O, Bjoras M, Slupphaug G, Seeberg E, Krokan HE. 2003. Human and bacterial oxidative demethylases repair alkylation damage in both RNA and DNA. Nature 421:859-863. doi:10.1038/nature01388.1.

Abernathy E, Glaunsinger B. 2015. Emerging roles for RNA degradation in viral replication and antiviral defense. Virology 479-480:600-608. doi:https://doi.org/10.1016/j.virol.2015.02.007

Aebi M, Kirchner G, Chen JY, Vijayraghavan U, Jacobson A, Martin NC, Abelson J. 1990. Isolation of a temperature-sensitive mutant with an altered tRNA nucleotidyltransferase and cloning of the gene encoding tRNA nucleotidyltransferase in the yeast Saccharomyces cerevisiae. J Biol Chem 265:16216-16220.

Ahn SH, Kim M, Buratowski S. 2004. Phosphorylation of serine 2 within the RNA polymerase II C-terminal domain couples transcription and 3' end processing. Mol Cell 13:67-76. doi:https://doi.org/10.1016/S1097-2765(03)00492-1

Anders S, Pyl PT, Huber W. 2015. HTSeq--a Python framework to work with highthroughput sequencing data. Bioinformatics 31:166-169. doi:10.1093/bioinformatics/btu638

Anderson JS, Parker RP. 1998. The 3' to 5' degradation of yeast mRNAs is a general mechanism for mRNA turnover that requires the SKI2 DEVH box protein and 3' to 5' exonucleases of the exosome complex. EMBO J 17:1497-1506. doi:10.1093/emboj/17.5.1497

Ansari A, Hampsey M. 2005. A role for the CPF 3'-end processing machinery in RNAP II-dependent gene looping. Genes Dev 19:2969-2978. doi:10.1101/gad.1362305

Araki Y, Takahashi S, Kobayashi T, Kajiho H, Hoshino SI, Katada T. 2001. Ski7p G protein interacts with the exosome and the ski complex for 3'-to-5' mRNA decay in yeast. EMBO J 20:4684-4693. doi:10.1093/emboj/20.17.4684

Arango D, Sturgill D, Alhusaini N, Dillman AA, Sweet TJ, Hanson G, Hosogane M, Sinclair WR, Nanan KK, Mandler MD, Fox SD, Zengeya TT, Andresson T, Meier JL, Coller J, Oberdoerffer S. 2018. Acetylation of Cytidine in mRNA Promotes Translation Efficiency. Cell 175:1872-1886.e24. doi:10.1016/j.cell.2018.10.030 Arigo JT, Eyler DE, Carroll KL, Corden JL. 2006. Termination of Cryptic Unstable 
Transcripts Is Directed by Yeast RNA-Binding Proteins Nrd1 and Nab3. Mol Cell 23:841-851. doi:10.1016/j.molcel.2006.07.024

Arimbasseri AG, Blewett NH, Iben JR, Lamichhane TN, Cherkasova V, Hafner M, Maraia RJ. 2015. RNA Polymerase III Output Is Functionally Linked to tRNA Dimethyl-G26 Modification. PLoS Genet 11:e1005671. doi:10.1371/journal.pgen.1005671

Arimbasseri AG, Iben J, Wei F, Rijal K, Tomizawa K, Hafner M, Maraia RJ. 2016. Evolving specificity of tRNA 3-methyl-cytidine-32 ( m 3 C32 ) modification : a subset of tRNAs Ser requires N 6 -isopentenylation of A37. RNA 32:1-11. doi:10.1261/rna.056259.116.5

Arts GJ, Kuersten S, Romby P, Ehresmann B, Mattaj IW. 1998. The role of exportin-t in selective nuclear export of mature tRNAs. EMBO $\mathrm{J}$ 17:7430-7441. doi:10.1093/emboj/17.24.7430

Asakura T, Sasaki T, Nagano F, Satoh a, Obaishi H, Nishioka H, Imamura H, Hotta K, Tanaka K, Nakanishi H, Takai Y. 1998. Isolation and characterization of a novel actin filament-binding protein from Saccharomyces cerevisiae. Oncogene 16:121-130. doi:10.1038/sj.onc.1201487

Ashe MP, Long SK De, Sachs AB. 2000. Glucose Depletion Rapidly Inhibits Translation Initiation in Yeast. Mol Biol Cell 11:833-848.

Auffinger P, Westhof E. 2001. An extended structural signature for the tRNA anticodon loop. RNA 7:334-341. doi:10.1017/S1355838201002382

Auffinger P, Westhof E. 1999. Singly and bifurcated hydrogen-bonded base-pairs in tRNA anticodon hairpins and ribozymes. J Mol Biol 292:467-483. doi:10.1006/jmbi.1999.3080

Baejen C, Andreani J, Torkler P, Battaglia S, Schwalb B, Lidschreiber M, Maier KC, Boltendahl A, Rus P, Esslinger S, Söding J, Cramer P. 2017. Genome-wide Analysis of RNA Polymerase II Termination at Protein-Coding Genes. Mol Cell 38-49. doi:10.1016/j.molcel.2017.02.009

Baejen C, Torkler P, Gressel S, Essig K, Söding J, Cramer P. 2014. Transcriptome maps of mRNP biogenesis factors define pre-mRNA recognition. Mol Cell 745757. doi:10.1016/j.molcel.2014.08.005

Baker KE, Parker R. 2004. Nonsense-mediated mRNA decay: terminating erroneous gene expression. Curr Opin Cell Biol 16:293-299. doi:10.1016/j.ceb.2004.03.003

Bakin A, Ofengand J. 1993. Four newly located pseudouridylate residues in Escherichia coli 23S ribosomal RNA are all at the peptidyltransferase center: Analysis by the application of a new sequencing technique. Biochemistry 
32:9754-9762. doi:10.1021/bi00088a030

Battaglia S, Lidschreiber M, Baejen C, Torkler P, Vos SM, Cramer P. 2017. RNAdependent chromatin association of transcription elongation factors and Pol II CTD kinases. Elife. doi:10.7554/eLife.25637

Beilharz TH, Preiss T. 2007. Widespread use of poly ( A ) tail length control to accentuate expression of the yeast transcriptome. RNA 13:982-997. doi:10.1261/rna.569407

Björk GR, Jacobsson K, Nilsson K, Johansson MJO, Byström AS, Persson OP. 2001. A primordial tRNA modification required for the evolution of life? EMBO J 20:231-239. doi:10.1093/emboj/20.1.231

Boeck R, Tarun SJ, Rieger M, Deardorff JA, Muller-Auer S, Sachs AB. 1996. The yeast Pan2 protein is required for poly $(A)$-binding protein-stimulated poly $(A)$ nuclease activity. J Biol Chem 271:432-438.

Bonneau F, Basquin J, Ebert J, Lorentzen E, Conti E. 2009. The yeast exosome functions as a macromolecular cage to channel RNA substrates for degradation. Cell 139:547-559. doi:10.1016/j.cell.2009.08.042

Booth MJ, Marsico G, Bachman M, Beraldi D. 2014. Quantitative sequencing of 5formylcytosine in DNA at single-base resolution. Nat Chem 6:435-440. doi:10.1038/nchem.1893

Bresson S, Tuck A, Staneva D, Tollervey D, Bresson S, Tuck A, Staneva D, Tollervey D. 2017. Nuclear RNA Decay Pathways Aid Rapid Remodeling of Gene Expression in Yeast Article Nuclear RNA Decay Pathways Aid Rapid Remodeling of Gene Expression in Yeast. Mol Cell 65:787-800.e5. doi:10.1016/j.molcel.2017.01.005

Brown CE, Sachs AB. 1998. Poly(A) tail length control in Saccharomyces cerevisiae occurs by message-specific deadenylation. Mol Cell Biol 18:6548-6559.

Brown JT, Bai X, Johnson AW. 2000. The yeast antiviral proteins Ski2p, Ski3p, and Ski8p exist as a complex in vivo. RNA 6:449-457. doi:10.1017/S1355838200991787

Buratowski S. 2003. The CTD code. Nat Struct Biol 10:679-680. doi:10.1038/nsb0903-679

Cao D, Parker R. 2003. Computational modeling and experimental analysis of nonsense-mediated decay in yeast. Cell 113:533-545.

Capone JP, Sharp PA, RajBhandary UL. 1985. Amber, ochre and opal suppressor tRNA genes derived from a human serine tRNA gene. EMBO J 4:213-21.

Caponigro G, Muhlrad D, Parker R. 1993. A small segment of the MAT alpha 1 transcript promotes mRNA decay in Saccharomyces cerevisiae: a stimulatory 
role for rare codons. Mol Cell Biol 13:5141-5148.

Caponigro G, Parker R. 1995. Multiple functions for the poly (A)-binding protein in mRNA decapping and deadenylation in yeast. Genes Dev 9:2421-2432. doi:10.1101/gad.9.19.2421

Carlile TM, Rojas-duran MF, Zinshteyn B, Shin H, Bartoli KM, Gilbert W V. 2014. Pseudouridine profiling reveals regulated mRNA pseudouridylation in yeast and human cells. Nature 515:143-146. doi:10.1038/nature13802

Celik A, Baker R, He F, Jacobson A. 2017. High-resolution profiling of NMD targets in yeast reveals translational fidelity as a basis for substrate selection. RNA 23:735-748. doi:10.1261/rna.060541.116

Chakrabarti S, Jayachandran U, Bonneau F, Fiorini F, Basquin C, Domcke S, Le Hir H, Conti E. 2011. Molecular mechanisms for the RNA-dependent ATPase activity of Upf1 and its regulation by Upf2. Mol Cell 41:693-703. doi:10.1016/j.molcel.2011.02.010

Chan PP, Lowe TM. 2009. GtRNAdb: a database of transfer RNA genes detected in genomic sequence. Nucleic Acids Res 37:D93-7. doi:10.1093/nar/gkn787

Chawla G, Sokol NS. 2014. ADAR mediates differential expression of polycistronic microRNAs. Nucleic Acids Res 42:5245-5255. doi:10.1093/nar/gku145

Chen C, Huang B, Eliasson M, Ryde'n P BA. 2011. Elongator Complex Influences Telomeric Gene Silencing and DNA Damage Response by Its Role in Wobble Uridine tRNA Modification. PLoS One 7:1-11. doi:10.1371/journal.pgen.1002258

Chen K, Lu Z, Wang X, Fu Y, Luo G-Z, Liu N, Han D, Dominissini D, Dai Q, Pan T, He C. 2015. High-resolution N(6) -methyl adenosine (m(6) A) map using photocrosslinking-assisted m(6) A sequencing. Angew Chem Int Ed Engl 54:1587-90. doi:10.1002/anie.201410647

Chen P, Jager G, Zheng B. 2010. Transfer RNA modifications and genes for modifying enzymes in Arabidopsis thaliana. BMC Plant Biol 10:201. doi:10.1186/1471-2229-10-201

Chimnaronk S, Suzuki Tateki, Manita T, Ikeuchi Y, Yao M, Suzuki Tsutomu, Tanaka I. 2009. RNA helicase module in an acetyltransferase that modifies a specific tRNA anticodon. EMBO J 28:1-12. doi:10.1038/emboj.2009.69

Cho EJ, Takagi T, Moore CR, Buratowski S. 1997. mRNA capping enzyme is recruited to the transcription complex by phosphorylation of the RNA polymerase II carboxy-terminal domain. Genes Dev 11:3319-3326.

Cho H, Kim KM, Kim YK. 2009. Human proline-rich nuclear receptor coregulatory protein 2 mediates an interaction between mRNA surveillance machinery and 
decapping complex. Mol Cell 33:75-86. doi:10.1016/j.molcel.2008.11.022

Chou H, Donnard E, Gustafsson HT, Garber M, Rando OJ, Chou H, Donnard E, Gustafsson HT, Garber M, Rando OJ. 2017. Transcriptome-wide Analysis of Roles for tRNA Modifications in Translational Regulation. Mol Cell 68:978992.e4. doi:10.1016/j.molcel.2017.11.002

Chowdhury A, Mukhopadhyay J, Tharun S. 2007. The decapping activator Lsm1p$7 p-P a t 1 p$ complex has the intrinsic ability to distinguish between oligoadenylated and polyadenylated RNAs. RNA 13:998-1016. doi:10.1261/rna.502507

Clark WC, Evans ME, Dominissini D, Zheng G, Pan T. 2016. tRNA base methylation identification and quantification via high-throughput sequencing. RNA 22:17711784. doi:10.1261/rna.056531.116

Colgan DF, Manley JL. 1997. Mechanism and regulation of mRNA polyadenylation. Genes Dev 11:2755-2766.

Coots RA, Liu X, Coots RA, Liu X, Mao Y, Dong L, Zhou J, Wan J, Zhang X. 2017. m6A Facilitates elF4F-Independent mRNA Translation. Mol Cell 68:504-514.e7. doi:10.1016/j.molcel.2017.10.002

Corden JL, Cadena DL, Ahearn JMJ, Dahmus ME. 1985. A unique structure at the carboxyl terminus of the largest subunit of eukaryotic RNA polymerase II. Proc Natl Acad Sci U S A 82:7934-7938.

Core LJ, Waterfall JJ, Lis JT. 2008. Nascent RNA sequencing reveals widespread pausing and divergent initiation at human promoters. Science 322:1845-1848. doi:10.1126/science.1162228

Creamer TJ, Darby MM, Jamonnak N, Schaughency P, Hao H, Wheelan SJ, Corden JL. 2011. Transcriptome-wide binding sites for components of the Saccharomyces cerevisiae non-poly(A) termination pathway: Nrd1, Nab3, and Sen1. PLoS Genet 7. doi:10.1371/journal.pgen.1002329

Cribbs DL, Gillam IC, Tener GM. 1987. Nucleotide sequences of three tRNASer from Drosophila melanogaster reading the six serine codons. J Mol Biol 197:389395. doi:10.1016/0022-2836(87)90552-3

Crick F. 1970. Central Dogma of Molecular Biology. Nature 227:561-563. doi:10.1038/227561a0

D'Silva SD, Haider SJ, Phizicky EM. 2011. A domain of the actin binding protein Abp140 is the yeast methyltransferase responsible for 3-methylcytidine modification in the tRNA anti-codon loop. RNA 1100-1110. doi:10.1261/rna.2652611.reading

Delan-Forino C, Schneider C, Tollervey D. 2017. Transcriptome-wide analysis of 
alternative routes for RNA substrates into the exosome complex. PLoS Genet 13:e1006699. doi:10.1371/journal.pgen.1006699

Delatte B, Wang F, Ngoc LV, Collignon E, Bonvin E, Deplus R, Calonne E, Hassabi B, Putmans P, Awe S, Wetzel C, Kreher J, Soin R, Creppe C, Limbach PA, Gueydan C, Kruys VV, Brehm A, Minakhina S, Defrance M, Steward R, Fuks FF. 2016. RNA biochemistry. Transcriptome-wide distribution and function of RNA hydroxymethylcytosine. Science 351:282-285. doi:10.1126/science.aac5253

Demeshkina N, Jenner L, Yusupova G, Yusupov M. 2010. Interactions of the ribosome with mRNA and tRNA. Curr Opin Struct Biol 20:325-332. doi:10.1016/j.sbi.2010.03.002

Dieci G, Sentenac A. 1996. Facilitated recycling pathway for RNA polymerase III. Cell 84:245-252.

Dobin A, Gingeras TR. 2015. Mapping RNA-seq Reads with STAR. Curr Protoc Bioinforma 51:11.14.1-19. doi:10.1002/0471250953.bi1114s51

Doma MK, Parker R. 2007. RNA quality control in eukaryotes. Cell 131:660-668. doi:10.1016/j.cell.2007.10.041

Dominissini D, Moshitch-Moshkovitz S, Schwartz S, Salmon-Divon M, Ungar L, Osenberg S, Cesarkas K, Jacob-Hirsch J, Amariglio N, Kupiec M, Sorek R, Rechavi G. 2012. Topology of the human and mouse m6A RNA methylomes revealed by m6A-seq. Nature 485:201-206. doi:10.1038/nature11112

Dominissini D, Nachtergaele S, Moshitch-Moshkovitz S, Peer E, Kol N, Ben-Haim MS, Dai Q, Di Segni A, Salmon-Divon M, Clark WC, Zheng G, Pan T, Solomon O, Eyal E, Hershkovitz V, Han D, Dore LC, Amariglio N, Rechavi G, He C. 2016. The dynamic N(1)-methyladenosine methylome in eukaryotic messenger RNA. Nature 530:441-446. doi:10.1038/nature16998

Dubin DT, Taylor RH. 1975. The methylation state of poly A-containing messenger RNA from cultured hamster cells. Nucleic Acids Res 2:1653-1668.

Duncan T, Trewick SC, Koivisto P, Bates PA, Lindahl T, Sedgwick B. 2002. Reversal of DNA alkylation damage by two human dioxygenases. Proc Natl Acad Sci U S A 99:16660-16665. doi:10.1073/pnas.262589799

Edelheit S, Schwartz S, Mumbach MR, Wurtzel O, Sorek R. 2013. Transcriptomewide mapping of 5-methylcytidine RNA modifications in bacteria, archaea, and yeast reveals m5C within archaeal mRNAs. PLoS Genet 9:e1003602. doi:10.1371/journal.pgen.1003602

Engel M, Eggert C, Kaplick PM, Eder M, Röh S, Tietze L, Namendorf C, Arloth J, Weber P, Rex-Haffner M, Geula S, Jakovcevski M, Hanna JH, Leshkowitz D, 
Uhr M, Wotjak CT, Schmidt M V, Deussing JM, Binder EB, Chen A. 2018. The Role of $\mathrm{m}(6) \mathrm{A} / \mathrm{m}-\mathrm{RNA}$ Methylation in Stress Response Regulation. Neuron 99:389-403.e9. doi:10.1016/j.neuron.2018.07.009

Engel SR, Dietrich FS, Fisk DG, Binkley G, Balakrishnan R, Costanzo MC, Dwight SS, Hitz BC, Karra K, Nash RS, Weng S, Wong ED, Lloyd P, Skrzypek MS, Miyasato SR, Simison M, Cherry JM. 2013. The reference genome sequence of Saccharomyces cerevisiae: then and now. G3 (Bethesda) 4:389-398. doi:10.1534/g3.113.008995

Esberg A, Huang B, Johansson MJO, Bystro AS. 2006. Elevated Levels of Two tRNA Species Bypass the Requirement for Elongator Complex in Transcription and Exocytosis 139-148. doi:10.1016/j.molcel.2006.07.031

Falk S, Weir JR, Hentschel J, Reichelt P, Bonneau F, Conti E. 2014. The Molecular Architecture of the TRAMP Complex Reveals the Organization and Interplay of Its Two Catalytic Activities. Mol Cell 55:856-867. doi:10.1016/j.molcel.2014.07.020

Farabaugh PJ, Kramer E, Vallabhaneni H, Raman A. 2006. Evolution of +1 programmed frameshifting signals and frameshift-regulating tRNAs in the order saccharomycetales. J Mol Evol 63:545-561. doi:10.1007/s00239-005-0311-0

Finoux A-L, Seraphin B. 2006. In vivo targeting of the yeast Pop2 deadenylase subunit to reporter transcripts induces their rapid degradation and generates new decay intermediates. J Biol Chem 281:25940-25947. doi:10.1074/jbc.M600132200

Fong N, Bentley DL. 2001. Capping, splicing, and 3' processing are independently stimulated by RNA polymerase II: different functions for different segments of the CTD. Genes Dev 15:1783-1795. doi:10.1101/gad.889101

Fox EJ, Reid-Bayliss KS, Emond MJ, Loeb LA. 2014. Accuracy of Next Generation Sequencing Platforms. Next Gener Seq App/ 1:1000106. doi:10.4172/jngsa.1000106

Franks TM, Lykke-Andersen J. 2008. The Control of mRNA Decapping and P-Body Formation. Mol Cell 32:605-615. doi:10.1016/j.molcel.2008.11.001

Franks TM, Singh G, Lykke-Andersen J. 2010. Upf1 ATPase-dependent mRNP disassembly is required for completion of nonsense- mediated mRNA decay. Cell 143:938-950. doi:10.1016/j.cell.2010.11.043

Frye M, Jaffrey SR, Pan T, Rechavi G, Suzuki T. 2016. RNA modifications: what have we learned and where are we headed? Nat Rev Genet 17:365.

Fustin J-M, Doi M, Yamaguchi Y, Hida H, Nishimura S, Yoshida M, Isagawa T, Morioka MS, Kakeya H, Manabe I, Okamura H. 2013. RNA-methylation- 
dependent RNA processing controls the speed of the circadian clock. Cell 155:793-806. doi:10.1016/j.cell.2013.10.026

Gallie DR. 1991. The cap and poly(A) tail function synergistically to regulate mRNA translational efficiency. Genes Dev 5:2108-2116.

Gao Y, Selmer M, Dunham CM, Weixlbaumer A, Kelley AC, Ramakrishnan V. 2009. The Structure of the Ribosome with Elongation Factor G Trapped in the Posttranslocational State. Science 326:694 LP - 699. doi:10.1126/science.1179709

Gasch AP, Spellman PT, Kao CM, Carmel-Harel O, Eisen MB, Storz G, Botstein D, Brown PO. 2000. Genomic expression programs in the response of yeast cells to environmental changes. Mol Biol Cell 11:4241-4257. doi:10.1091/mbc.11.12.4241

Gasch AP, Werner-Washburne M. 2002. The genomics of yeast responses to environmental stress and starvation. Funct Integr Genomics 2:181-192. doi:10.1007/s10142-002-0058-2

Gerber A, Grosjean H, Melcher T, Keller W. 1998. Tad1p, a yeast tRNA-specific adenosine deaminase, is related to the mammalian pre-mRNA editing enzymes ADAR1 and ADAR2. EMBO J 17:4780-4789. doi:10.1093/emboj/17.16.4780

Gilmartin GM, Nevins JR. 1989. An ordered pathway of assembly of components required for polyadenylation site recognition and processing. Genes Dev 3:2180-2190.

Goetz AE, Wilkinson M. 2017. Stress and the nonsense-mediated RNA decay pathway. Cell Mol Life Sci 74:3509-3531. doi:10.1007/s00018-017-2537-6

Goldstrohm AC, Hook BA, Seay DJ, Wickens M. 2006. PUF proteins bind Pop2p to regulate messenger RNAs. Nat Struct Mol Biol 13:533-539. doi:10.1038/nsmb1100

Green R, Noller HF. 1997. Ribosomes and translation. Annu Rev Biochem 66:679716. doi:10.1146/annurev.biochem.66.1.679

Gressel S, Schwalb B, Decker TM, Qin W, Leonhardt H, Eick D, Cramer P. 2017. CDK9-dependent RNA polymerase II pausing controls transcription initiation. Elife 6. doi:10.7554/eLife.29736

Grosshans H, Simos G, Hurt E. 2000. Review: transport of tRNA out of the nucleusdirect channeling to the ribosome? J Struct Biol 129:288-294. doi:10.1006/jsbi.2000.4226

Grzechnik P, Kufel J. 2008. Polyadenylation Linked to Transcription Termination Directs the Processing of snoRNA Precursors in Yeast. Mol Cell 32:247-258. doi:10.1016/j.molcel.2008.10.003 
Hahn S. 2004. Structure and mechanism of the RNA polymerase II transcription machinery. Nat Struct Mol Biol 11:394-403. doi:10.1038/nsmb763

Halbach F, Reichelt P, Rode M, Conti E. 2013. The yeast ski complex: Crystal structure and rna channeling to the exosome complex. Cell 154:814-826. doi:10.1016/j.cell.2013.07.017

Han D, Liu J, Chen C, Dong L, Liu Yi, Chang R, Huang X, Liu Yuanyuan, Wang J, Dougherty U, Bissonnette MB, Shen B, Weichselbaum RR, Xu MM, He C. 2019. Anti-tumour immunity controlled through mRNA m6A methylation and YTHDF1 in dendritic cells. Nature 566:270-274. doi:10.1038/s41586-019-0916-x

Han LU, Marcus E, D'Silva S, Phizicky EM, Silva SD, Phizicky EM, D'Silva S, Phizicky EM. 2017. S. cerevisiae Trm140 has two recognition modes for 3methylcytidine modification of the anticodon loop of tRNA substrates. RNA 23:406-419. doi:10.1261/rna.059667.116

Hanson G, Coller J. 2018. Translation and Protein Quality Control: Codon optimality, bias and usage in translation and mRNA decay. Nat Rev Mol Cell Biol 19:2030. doi:10.1038/nrm.2017.91

Hantsche M, Cramer P. 2016. The Structural Basis of Transcription: 10 Years After the Nobel Prize in Chemistry. Angew Chemie Int Ed 55:15972-15981. doi:10.1002/anie.201608066

He PC, He C. 2019. mRNA acetylation: a new addition to the epitranscriptome. Cell Res 29:91-92. doi:10.1038/s41422-018-0135-2

Heck AM, Wilusz J. 2018. The Interplay between the RNA Decay and Translation Machinery in Eukaryotes. Cold Spring Harb Perspect Biol a032839. doi:10.1101/cshperspect.a032839

Hellen CUT. 2018. Translation Termination and Ribosome Recycling in Eukaryotes. Cold Spring Harb Perspect Biol 10. doi:10.1101/cshperspect.a032656

Heo D, Yoo I, Kong J, Lidschreiber M, Mayer A, Choi B-Y, Hahn Y, Cramer P, Buratowski S, Kim M. 2013. The RNA polymerase II C-terminal domaininteracting domain of yeast $\mathrm{Nrd} 1$ contributes to the choice of termination pathway and couples to RNA processing by the nuclear exosome. J Biol Chem 288:36676-36690. doi:10.1074/jbc.M113.508267

Hilgers V, Teixeira D, Parker R. 2006. Translation-independent inhibition of mRNA deadenylation during stress in Saccharomyces cerevisiae. RNA 12:1835-1845. doi:10.1261/rna.241006

Hirose Y, Manley JL. 2000. RNA polymerase II and the integration of nuclear events. Genes Dev 14:1415-1429. doi:10.1101/gad.14.12.1415

Hoernes TP, Clementi N, Faserl K, Glasner H, Erlacher MD, Breuker K, Lindner H, 
Alexander H. 2016. Nucleotide modifications within bacterial messenger RNAs regulate their translation and are able to rewire the genetic code. Nucleic Acids Res 44:852-862. doi:10.1093/nar/gkv1182

Hongay CF, Orr-weaver TL. 2011. Drosophila Inducer of MEiosis 4 ( IME4 ) is required for Notch signaling during oogenesis. Proc Natl Acad Sci 108:1485514860. doi:10.1073/pnas. 1111577108

Hopper AK. 2013. Transfer RNA post-transcriptional processing, turnover, and subcellular dynamics in the yeast Saccharomyces cerevisiae. Genetics 194:4367. doi:10.1534/genetics.112.147470

Houseley J, Tollervey D. 2009. The Many Pathways of RNA Degradation. Cell 136:763-776. doi:10.1016/j.cell.2009.01.019

Hsu PJ, Shi H, He C. 2017. Epitranscriptomic influences on development and disease. Genome Biol 18:1-9. doi:10.1186/s13059-017-1336-6

Huang $\mathrm{H}$, Weng H, Zhou K, Wu T, Zhao BS, Sun Mingli, Chen Z, Deng X, Xiao G, Auer F, Klemm L, Wu H, Zuo Z, Qin X, Dong Y, Zhou Y, Qin H, Tao S, Du J, Liu J, Lu Z, Yin H, Mesquita A, Yuan CL, Hu Y-C, Sun W, Su R, Dong L, Shen C, Li C, Qing Y, Jiang X, Wu X, Sun Miao, Guan J-L, Qu L, Wei M, Müschen M, Huang G, He C, Yang J, Chen J. 2019. Histone H3 trimethylation at lysine 36 guides m6A RNA modification co-transcriptionally. Nature. doi:10.1038/s41586019-1016-7

Huang L, Wilkinson MF. 2012. Regulation of nonsense-mediated mRNA decay. Wiley Interdiscip Rev RNA 3:807-828. doi:10.1002/wrna.1137

Huang Y, Carmichael GG. 1996. Role of polyadenylation in nucleocytoplasmic transport of mRNA. Mol Cell Biol 16:1534-1542.

Huber SM, Delft P Van, Mendil L, Bachman M, Smollett K, Werner F, Miska EA, Balasubramanian S. 2015. Formation and Abundance of 5Hydroxymethylcytosine in RNA. Chembiochem 16:752-755. doi:10.1002/cbic.201500013

Huch S, Nissan T. 2014. Interrelations between translation and general mRNA degradation in yeast. Wiley Interdiscip Rev RNA 5:747-763. doi:10.1002/wrna.1244

Hussain S. 2018. Native RNA-Sequencing Throws its Hat into the Transcriptomics Ring. Trends Biochem Sci 43:225-227. doi:10.1016/j.tibs.2018.02.007 Ingolia NT, Ghaemmaghami S, Newman JRS, Weissman JS. 2009. Genome-Wide Analysis in Vivo of Translation with Nucleotide Resolution Using Ribosome Profiling. Science 324:218-224.

Ito S, Horikawa S, Suzuki TTT, Kawauchi H, Tanaka Y, Suzuki TTT, Suzuki TTT. 
2014. Human NAT10 is an ATP-dependent RNA acetyltransferase responsible for N4-acetylcytidine formation in $18 \mathrm{~S}$ ribosomal RNA (rRNA). J Biol Chem 289:35724-35730. doi:10.1074/jbc.C114.602698

Jackson RJ, Hellen CUT, Pestova T V. 2012. Termination and Post-Termination Events in Eukaryotic Translation. Adv Protein Chem Struct Biol 86:45-93ab. Jantsch MF, Schaefer MR. 2019. Mining the Epitranscriptome: Detection of RNA editing and RNA modifications. Methods 156:1-4. doi:https://doi.org/10.1016/j.ymeth.2019.02.016

Jelinsky SA, Samson LD. 1999. Global response of Saccharomyces cerevisiae to an alkylating agent. Proc Natl Acad Sci U S A 96:1486-1491.

Jia G, Fu Y, Zhao X, Dai Q, Zheng G, Yang Y, Yi C, Lindahl T, Pan T, Yang Y-G, He C. 2011. N6-methyladenosine in nuclear RNA is a major substrate of the obesity-associated FTO. Nat Chem Biol 7:885-887. doi:10.1038/nchembio.687 Jiang H, Lei R, Ding S-W, Zhu S. 2014. Skewer: a fast and accurate adapter trimmer for next-generation sequencing paired-end reads. BMC Bioinformatics 15:182. doi:10.1186/1471-2105-15-182

Jiao X, Xiang S, Oh C, Martin CE, Tong L, Kiledjian M. 2010. Identification of a quality-control mechanism for mRNA 5'-end capping. Nature 467:608-611. Johansson MJO, Esberg A, Huang B, Björk GR, Byström AS. 2008. Eukaryotic Wobble Uridine Modifications Promote a Functionally Redundant Decoding System . Mol Cell Biol 28:3301-3312. doi:10.1128/MCB.01542-07

Johnson SJ, Jackson RN. 2013. Ski2-like RNA helicase structures: common themes and complex assemblies. RNA Biol 10:33-43. doi:10.4161/rna.22101

Jona G, Choder M, Gileadi O. 2000. Glucose starvation induces a drastic reduction in the rates of both transcription and degradation of mRNA in yeast. Biochim Biophys Acta - Gene Struct Expr 1491:37-48. doi:https://doi.org/10.1016/S0167-4781(00)00016-6

Karam R, Lou C-H, Kroeger H, Huang L, Lin JH, Wilkinson MF. 2015. The unfolded protein response is shaped by the NMD pathway. EMBO Rep 16:599-609. doi:10.15252/embr.201439696

Karam R, Wengrod J, Gardner LB, Wilkinson MF. 2013. Regulation of nonsensemediated mRNA decay: implications for physiology and disease. Biochim Biophys Acta 1829:624-633. doi:10.1016/j.bbagrm.2013.03.002

Karijolich J, Yu Y-T. 2011. Converting nonsense codons into sense codons by targeted pseudouridylation. Nature 474:395-398. doi:10.1038/nature10165

Kariko K, Muramatsu H, Welsh FA, Ludwig J, Kato H, Akira S, Weissman D. 2008. Incorporation of pseudouridine into mRNA yields superior nonimmunogenic 
vector with increased translational capacity and biological stability. Mol Ther 16:1833-1840. doi:10.1038/mt.2008.200

Keith G. 1984. TThe primary structures of two arginine tRNAs (anticodons C-C-U and $\mathrm{mcm} 5 \mathrm{a} 2 \mathrm{U}-\mathrm{C}-\mathrm{psi}$ ) and of glutamine tRNA (anticodon C-U-G) from bovine liver. Nucleic Acids Res 12:2543-2548.

Kilchert C, Spang A. 2011. Cotranslational transport of ABP140 mRNA to the distal pole of S. cerevisiae. EMBO J 30:3567-3580. doi:10.1038/emboj.2011.247

Kilchert C, Wittmann S, Vasiljeva L. 2016. The regulation and functions of the nuclear RNA exosome complex. Nat Rev Mol Cell Biol 17:227.

Kizer KO, Phatnani HP, Shibata Y, Hall H, Greenleaf AL, Strahl BD, lol MOLCELLB. 2005. A Novel Domain in Set2 Mediates RNA Polymerase II Interaction and Couples Histone H3 K36 Methylation with Transcript Elongation. Mol Cell Biol 25:3305-3316. doi:10.1128/MCB.25.8.3305

Klose RJ, Bird AP. 2006. Genomic DNA methylation: the mark and its mediators. Trends Biochem Sci 31:89-97. doi:10.1016/j.tibs.2005.12.008

Kowalinski E, Kögel A, Ebert J, Reichelt P, Stegmann E, Habermann B, Conti E. 2016. Structure of a Cytoplasmic 11-Subunit RNA Exosome Complex. Mol Cell 63:125-134. doi:10.1016/j.molcel.2016.05.028

Kretschmer J, Rao H, Hackert P, Sloan KE, Höbartner C, Bohnsack MT. 2018. The m6A reader protein YTHDC2 interacts with the small ribosomal subunit and the 5 ' -3 ' exoribonuclease XRN1. RNA 24:1339-1350. doi:10.1261/rna.064238.117

Kruk JA, Dutta A, Fu J, Gilmour DS, Reese JC. 2011. The multifunctional Ccr4-Not complex directly promotes transcription elongation. Genes Dev 25:581-593. doi:10.1101/gad.2020911

Lai T, Cho H, Liu Z, Bowler MW, Piao S, Parker R, Kim YK, Song H. 2012. Structural basis of the PNRC2-mediated link between mrna surveillance and decapping. Structure 20:2025-2037. doi:10.1016/j.str.2012.09.009

Lebreton A, Seraphin B. 2008. Exosome-mediated quality control: substrate recruitment and molecular activity. Biochim Biophys Acta 1779:558-565. doi:10.1016/j.bbagrm.2008.02.003

Lee D-H, Jin S-G, Cai S, Chen Y, Pfeifer GP, O'Connor TR. 2005. Repair of methylation damage in DNA and RNA by mammalian AlkB homologues. $J$ Biol Chem 280:39448-39459. doi:10.1074/jbc.M509881200

Lee S, Kim J. 2016. NGS-based deep bisulfite sequencing. MethodsX 3:1-7. doi:10.1016/j.mex.2015.11.008

Lence T, Akhtar J, Bayer M, Schmid K, Spindler L, Ho CH, Kreim N, Andrade- 
Navarro MA, Poeck B, Helm M, Roignant J-Y. 2016. m(6)A modulates neuronal functions and sex determination in Drosophila. Nature 540:242-247. doi:10.1038/nature20568

Li A, Chen Y-S, Ping X-L, Yang X, Xiao W, Yang Y, Sun H-Y, Zhu Q, Baidya P, Wang X, Bhattarai DP, Zhao Y-L, Sun B-F, Yang Y-G. 2017. Cytoplasmic m6A reader YTHDF3 promotes mRNA translation. Cell Res 27:444.

Li F, Zhao D, Wu J, Shi Y. 2014. Structure of the YTH domain of human YTHDF2 in complex with an $\mathrm{m}(6) \mathrm{A}$ mononucleotide reveals an aromatic cage for $\mathrm{m}(6) \mathrm{A}$ recognition. Cell Res. doi:10.1038/cr.2014.153

Li H, Handsaker B, Wysoker A, Fennell T, Ruan J, Homer N, Marth G, Abecasis G, Durbin R. 2009. The Sequence Alignment/Map format and SAMtools.

Bioinformatics 25:2078-2079. doi:10.1093/bioinformatics/btp352

Li T, Shi Y, Wang P, Guachalla LM, Sun B, Joerss T, Chen Y-S, Groth M, Krueger A, Platzer M, Yang Y-G, Rudolph KL, Wang Z-Q. 2015. Smg6/Est1 licenses embryonic stem cell differentiation via nonsense-mediated mRNA decay. EMBO J 34:1630-1647. doi:10.15252/embj.201489947

Li X, Xiong X. 2017. Base-Resolution Mapping Reveals Distinct m 1 A Methylome in Nuclear- and Mitochondrial-Encoded Technology Base-Resolution Mapping Reveals Distinct m 1 A Methylome in Nuclear- and Mitochondrial-Encoded Transcripts. Mol Cell 68:993-1005.e9. doi:10.1016/j.molcel.2017.10.019

Li X, Xiong X, Wang K, Wang L, Shu X, Ma S, Yi C. 2016a. Transcriptome-wide mapping reveals reversible and dynamic N1-methyladenosine methylome. Nat Chem Biol 12:311-316. doi:10.1038/nchembio.2040

Li X, Xiong X, Yi C. 2016b. Epitranscriptome sequencing technologies: decoding RNA modifications. Nat Methods 14:23-31. doi:10.1038/nmeth.4110

Li X, Zhu P, Ma S, Song J, Bai J, Sun F, Yi C. 2015. Chemical pulldown reveals dynamic pseudouridylation of the mammalian transcriptome. Nat Chem Biol 11:592-597. doi:10.1038/nchembio.1836

Linder B, Grozhik A V, Olarerin-george AO, Meydan C, Mason CE, Jaffrey SR. 2015. Single-nucleotide-resolution mapping of $\mathrm{m} 6 \mathrm{~A}$ and $\mathrm{m} 6 \mathrm{Am}$ throughout the transcriptome. Nat Meth 12:767-772. doi:10.1038/nmeth.3453

Liu H, Begik O, Lucas MC, Christopher E, Schwartz S, Mattick JS, Smith MA, Novoa M. 2019. Accurate detection of m6A RNA modifications in native RNA sequences. bioRxiv. doi:https://doi.org/10.1101/525741

Liu J, Jia G. 2014. Methylation Modifications in Eukaryotic Messenger RNA. J Genet Genomics 41:21-33. doi:10.1016/j.jgg.2013.10.002

Liu N, Dai Q, Zheng G, He C, Parisien M, Pan T. 2015. N6-methyladenosine- 
dependent RNA structural switches regulate RNA-protein interactions. Nature 518:560-564. doi:10.1038/nature14234

Liu Q, Greimann JC, Lima CD. 2006. Reconstitution, activities, and structure of the eukaryotic RNA exosome. Cell 127:1223-1237. doi:10.1016/j.cell.2006.10.037

Lorenz C, Lünse CE, Mörl M. 2017. tRNA Modifications: Impact on Structure and Thermal Adaptation. Biomolecules 7:35. doi:10.3390/biom7020035

Losh JS, King AK, Bakelar J, Taylor L, Loomis J, Rosenzweig JA, Johnson SJ, Hoof A Van. 2015. Interaction between the RNA-dependent ATPase and poly(A) polymerase subunits of the TRAMP complex is mediated by short peptides and important for snoRNA processing. Nucleic Acids Res 43:1848-1858. doi:10.1093/nar/gkv005

Losson R, Lacroute F. 1979. Interference of nonsense mutations with eukaryotic messenger RNA stability. Proc Natl Acad Sci U S A 76:5134-5137.

Lou C-H, Dumdie J, Goetz A, Shum EY, Brafman D, Liao X, Mora-Castilla S, Ramaiah M, Cook-Andersen H, Laurent L, Wilkinson MF. 2016. NonsenseMediated RNA Decay Influences Human Embryonic Stem Cell Fate. Stem cell reports 6:844-857. doi:10.1016/j.stemcr.2016.05.008

Lovejoy AF, Riordan DP, Brown PO. 2014. Transcriptome-Wide Mapping of Pseudouridines: Pseudouridine Synthases Modify Specific mRNAs in S. cerevisiae. PLoS One 9:e110799. doi:10.1371/journal.pone.0110799

Luehr S, Hartmann H, Söding J. 2012. The XXmotif web server for eXhaustive, weight matriX-based motif discovery in nucleotide sequences. Nucleic Acids Res 40:W104-W109. doi:10.1093/nar/gks602

Lund E, Dahlberg JE. 1998. Proofreading and aminoacylation of tRNAs before export from the nucleus. Science 282:2082-2085.

Luo S, Tong L. 2014. Molecular basis for the recognition of methylated adenines in RNA by the eukaryotic YTH domain. Proc Natl Acad Sci U S A 111:1383413839. doi:10.1073/pnas.1412742111

Lykke-Andersen S, Brodersen DE, Jensen TH. 2009. Origins and activities of the eukaryotic exosome. J Cell Sci 122:1487-1494. doi:10.1242/jcs.047399

Lykke-Andersen S, Jensen TH. 2015. Nonsense-mediated mRNA decay: an intricate machinery that shapes transcriptomes. Nat Rev Mol Cell Biol 16:665-677. doi:10.1038/nrm4063

Marquardt S, Hazelbaker DZ, Buratowski S. 2011. Distinct RNA degradation pathways and 3' extensions of yeast non-coding RNA species. Transcription 2:145-154. doi:10.4161/trns.2.3.16298

McCracken S, Fong N, Rosonina E, Yankulov K, Brothers G, Siderovski D, Hessel A, 
Foster S, Shuman S, Bentley DL. 1997a. 5'-Capping enzymes are targeted to pre-mRNA by binding to the phosphorylated carboxy-terminal domain of RNA polymerase II. Genes Dev 11:3306-3318.

McCracken S, Fong N, Yankulov K, Ballantyne S, Pan G, Greenblatt J, Patterson SD, Wickens M, Bentley DL. 1997b. The C-terminal domain of RNA polymerase II couples mRNA processing to transcription. Nature 385:357-361. doi:10.1038/385357a0

Melton DA, De Robertis EM, Cortese R. 1980. Order and intracellular location of the events involved in the maturation of a spliced tRNA. Nature 284:143-148. Meyer KD, Patil DP, Zhou J, Zinoviev A, Skabkin MA, Elemento O, Pestova T V, Qian S-B, Jaffrey SR. 2015. 5' UTR m(6)A Promotes Cap-Independent Translation. Cell 163:999-1010. doi:10.1016/j.cell.2015.10.012

Mignone F, Gissi C, Liuni S, Pesole G. 2002. Untranslated regions of mRNAs. Genome Biol 3.

Miller C, Schwalb B, Maier K, Schulz D, Dümcke S, Zacher B, Mayer A, Sydow J, Marcinowski L, Dölken L, Martin DE, Tresch A, Cramer P. 2011. Dynamic transcriptome analysis measures rates of mRNA synthesis and decay in yeast. Mol Syst Biol 7. doi:10.1038/msb.2010.112

Miller JE, Reese JC. 2012. Ccr4-Not complex: The control freak of eukaryotic cells. Crit Rev Biochem Mol Biol 47:315-333. doi:10.3109/10409238.2012.667214 Milligan L, Decourty L, Saveanu C, Rappsilber J, Ceulemans H, Jacquier A, Tollervey D. 2008. A Yeast Exosome Cofactor, Mpp6, Functions in RNA Surveillance and in the Degradation of Noncoding RNA Transcripts. Mol Cell Biol 28:5446-5457. doi:10.1128/MCB.00463-08

Milligan L, Huynh-Thu VA, Delan-Forino C, Tuck A, Petfalski E, Lombraña R, Sanguinetti G, Kudla G, Tollervey D. 2016. Strand-specific, high-resolution mapping of modified RNA polymerase II. Mol Syst Biol 12:874. doi:10.15252/msb.20166869

Mitchell P, Petfalski E, Houalla R, Podtelejnikov A, Mann M, Tollervey D. 2003. Rrp47p is an exosome-associated protein required for the 3' processing of stable RNAs. Mol Cell Biol 23:6982-6992.

Mitchell P, Tollervey D. 2003. An NMD pathway in yeast involving accelerated deadenylation and exosome-mediated 3'-->5' degradation. Mol Cell 11:14051413.

Moore CL, Sharp PA. 1985. Accurate cleavage and polyadenylation of exogenous RNA substrate. Cell 41:845-855.

Morrissey JP, Deardorff JA, Hebron C, Sachs AB. 1999. Decapping of stabilized, 
polyadenylated mRNA in yeast pab1 mutants. Yeast 15:687-702.

Muhlrad D, Parker R. 1999. Aberrant mRNAs with extended 3' UTRs are substrates for rapid degradation by mRNA surveillance. RNA 5:1299-1307.

Muhlrad D, Parker R. 1994. Premature translational termination triggers mRNA decapping. Nature 370:578-581. doi:10.1038/370578a0

Murphy F V, Ramakrishnan V. 2004. Structure of a purine-purine wobble base pair in the decoding center of the ribosome. Nat Struct Mol Biol 11:1251-1252.

Murray SC, Serra Barros A, Brown DA, Dudek P, Ayling J, Mellor J. 2012. A preinitiation complex at the 3'-end of genes drives antisense transcription independent of divergent sense transcription. Nucleic Acids Res 40:2432-2444. doi:10.1093/nar/gkr1121

Nabet B, Roberts JM, Buckley DL, Paulk J, Dastjerdi S, Yang A, Leggett AL, Erb MA, Lawlor MA, Souza A, Scott TG, Vittori S, Perry JA, Qi J, Winter GE, Wong K-K, Gray NS, Bradner JE. 2018. The dTAG system for immediate and targetspecific protein degradation. Nat Chem Biol 14:431-441. doi:10.1038/s41589018-0021-8

Nechaev S, Adelman K. 2011. Pol II waiting in the starting gates: Regulating the transition from transcription initiation into productive elongation. Biochim Biophys Acta 1809:34-45. doi:10.1016/j.bbagrm.2010.11.001

Nedialkova DD, Leidel SA. 2015. Optimization of Codon Translation Rates via tRNA Modifications Maintains Proteome Integrity. Cell 161:1606-1618. doi:10.1016/j.cell.2015.05.022

Neil H, Malabat C, d'Aubenton-Carafa Y, Xu Z, Steinmetz LM, Jacquier A. 2009. Widespread bidirectional promoters are the major source of cryptic transcripts in yeast. Nature 457:1038-1042. doi:10.1038/nature07747

Nelson JO, Moore KA, Chapin A, Hollien J, Metzstein MM. 2016. Degradation of Gadd45 mRNA by nonsense-mediated decay is essential for viability. Elife $\mathbf{5}$. doi:10.7554/eLife.12876

Noma A, Yi S, Katoh T, Takai Y, Suzuki T. 2011. Actin-binding protein ABP140 is a methyltransferase for 3-methylcytidine at position 32 of tRNAs in Saccharomyces cerevisiae. RNA 17:1111-1119. doi:10.1261/rna.2653411.1968

Parker R. 2012. RNA degradation in Saccharomyces cerevisae. Genetics 191:671702. doi:10.1534/genetics.111.137265

Parker R, Song H. 2004. The enzymes and control of eukaryotic mRNA turnover. Nat Struct Mol Biol 11:121-127. doi:10.1038/nsmb724

Partial T, Digestion E, Ginsberg T, Rogg H. 1971. Nucleotide Sequences of Rat Liver Serine- tRNA and Derivation of its Total Primary Structure. Eur J Biochem 
21:249-257.

Pechmann S, Frydman J. 2013. Evolutionary conservation of codon optimality reveals hidden signatures of cotranslational folding. Nat Struct Mol Biol 20:237243. doi: $10.1038 / \mathrm{nsmb} .2466$

Pelechano V, Wei W, Steinmetz LM. 2013. Extensive transcriptional heterogeneity revealed by isoform profiling. Nature 497:127-131. doi:10.1038/nature12121

Pelechano V, Wei W, Steinmetz LMM. 2015. Widespread co-translational RNA decay reveals ribosome dynamics. Cell 161:1400-1412. doi:10.1016/j.cell.2015.05.008

Pendleton KE, Chen B, Liu K, Hunter O V, Xie Y, Tu BP, Conrad NK. 2017. The U6 snRNA m(6)A Methyltransferase METTL16 Regulates SAM Synthetase Intron Retention. Cell 169:824-835.e14. doi:10.1016/j.cell.2017.05.003

Pestova T V, Kolupaeva VG, Lomakin IB, Pilipenko E V, Shatsky IN, Agol VI, Hellen CUT. 2001. Molecular mechanisms of translation initiation in eukaryotes. Proc Natl Acad Sci 98:7029 LP - 7036. doi:10.1073/pnas.111145798

Phizicky EM, Hopper AK. 2010. tRNA biology charges to the front. Genes Dev 24:1832-1860. doi:10.1101/gad.1956510

Ping X-L, Sun B-F, Wang L, Xiao W, Yang X, Wang W-J, Adhikari S, Shi Y, Lv Y, Chen Y-S, Zhao X, Li A, Yang Y, Dahal U, Lou X-M, Liu X, Huang J, Yuan W-P, Zhu X-F, Cheng T, Zhao Y-L, Wang X, Rendtlew Danielsen JM, Liu F, Yang Y-

G. 2014. Mammalian WTAP is a regulatory subunit of the RNA N6methyladenosine methyltransferase. Cell Res 24:177-189. doi:10.1038/cr.2014.3

Powell LM, Wallis SC, Pease RJ, Edwards YH, Knott TJ, Scott J. 1987. A novel form of tissue-specific RNA processing produces apolipoprotein-B48 in intestine. Cell 50:831-840.

Presnyak V, Alhusaini N, Chen Y-H, Martin S, Morris N, Kline N, Olson S, Weinberg D, Baker KE, Graveley BR, Coller J. 2015. Codon Optimality Is a Major Determinant of mRNA Stability. Cell 160:1111-1124. doi:10.1016/J.CELL.2015.02.029

Proudfoot NJ, Furger A, Dye MJ. 2002. Integrating mRNA processing with transcription. Cell 108:501-512.

Radhakrishnan A, Chen YH, Martin S, Alhusaini N, Green R, Coller J. 2016. The DEAD-Box Protein Dhh1p Couples mRNA Decay and Translation by Monitoring Codon Optimality. Cell 167:122-132.e9. doi:10.1016/j.cell.2016.08.053

Rasmussen EB, Lis JT. 1993. In vivo transcriptional pausing and cap formation on three Drosophila heat shock genes. Proc Natl Acad Sci U S A 90:7923-7927. 
Rhee HS, Pugh BF. 2012. Genome-wide structure and organization of eukaryotic pre-initiation complexes. Nature 483:295-301. doi:10.1038/nature10799

Roth C, Torkler P. 2018. soedinglab/mockinbird: Degradation PAR-CLIP. doi:10.5281/ZENODO.1342555

Safra M, Nir R, Farouq D, Slutzkin IV. 2017a. TRUB1 is the predominant pseudouridine synthase acting on mammalian mRNA via a predictable and conserved code. Genome Res 27:393-406. doi:10.1101/gr.207613.116.5

Safra M, Sas-Chen A, Nir R, Winkler R, Nachshon A, Bar-Yaacov D, Erlacher M, Rossmanith W, Stern-Ginossar N, Schwartz S. 2017b. The m1A landscape on cytosolic and mitochondrial mRNA at single-base resolution. Nature 551:251255. doi:10.1038/nature24456

Sainsbury S, Bernecky C, Cramer P. 2015. Structural basis of transcription initiation by RNA polymerase II. Nat Rev Mol Cell Biol 16:129-143. doi:10.1038/nrm3952

Saletore Y, Meyer K, Korlach J, Vilfan ID, Jaffrey S, Mason CE. 2012. The birth of the Epitranscriptome: deciphering the function of RNA modifications. Genome Biol 13:175. doi:10.1186/gb-2012-13-10-175

Sarkar S, Hopper AK. 1998. tRNA nuclear export in saccharomyces cerevisiae: in situ hybridization analysis. Mol Biol Cell 9:3041-3055.

Schaeffer D, Tsanova B, Barbas A, Reis FP, Dastidar EG, Sanchez-Rotunno M, Arraiano CM, van Hoof A. 2009. The exosome contains domains with specific endoribonuclease, exoribonuclease and cytoplasmic mRNA decay activities. Nat Struct Mol Biol 16:56-62. doi:10.1038/nsmb.1528

Schibler U, Kelley DE, Perry RP. 1977. Comparison of methylated sequences in messenger RNA and heterogeneous nuclear RNA from mouse $L$ cells. $J$ Mol Biol 115:695-714.

Schmeing TM, Voorhees RM, Kelley AC, Gao Y-G, Murphy F V, Weir JR, Ramakrishnan V, Iv FVM, Weir JR, Ramakrishnan V. 2009. The Crystal Structure of the Ribosome Bound to EF-Tu and Aminoacyl-tRNA. Science 326:688 LP - 694. doi:10.1126/science.1179700

Schmeing TM, Voorhees RM, Kelley AC, Ramakrishnan V. 2011. How mutations in tRNA distant from the anticodon affect the fidelity of decoding. Nat Struct \&Amp; Mol Biol 18:432.

Schmidt C, Kowalinski E, Shanmuganathan V, Defenouillère Q, Braunger K, Heuer A, Pech M, Namane A, Berninghausen O, Fromont-Racine M, Jacquier A, Conti E, Becker T, Beckmann R. 2016. The cryo-EM structure of a ribosome-Ski2Ski3-Ski8 helicase complex. Science 354:1431-1433. doi:10.1126/science.aaf7520 
Schmidt K, Butler JS. 2013. Nuclear RNA surveillance: Role of TRAMP in controlling exosome specificity. Wiley Interdiscip Rev RNA 4:217-231. doi:10.1002/wrna.1155

Schneider C, Kudla G, Wlotzka W, Tuck A, Tollervey D. 2012. Transcriptome-wide Analysis of Exosome Targets. Mol Cell 48:422-433. doi:10.1016/j.molcel.2012.08.013

Schneider C, Tollervey D. 2013. Threading the barrel of the RNA exosome. Trends Biochem Sci 38:485-493. doi:10.1016/j.tibs.2013.06.013

Schulz D, Schwalb B, Kiesel A, Baejen C, Torkler P, Gagneur J, Soeding J, Cramer P. 2013. Transcriptome surveillance by selective termination of noncoding RNA synthesis. Cell 155:1075-1087. doi:10.1016/j.cell.2013.10.024

Schwalb B, Michel M, Zacher B, Fruhauf K, Demel C, Tresch A, Gagneur J, Cramer P. 2016. TT-seq maps the human transient transcriptome. Science 352:12251228. doi:10.1126/science.aad9841

Schwartz S. 2018. M1A within cytoplasmic mRNAs at single nucleotide resolution: A reconciled transcriptome-wide map. RNA.

Schwartz S, Agarwala SD, Mumbach MR, Jovanovic M, Mertins P, Shishkin A, Tabach Y, Mikkelsen TS, Satija R, Ruvkun G, Carr SA, Lander ES, Fink GR, Regev A. 2013. Resource High-Resolution Mapping Reveals a Conserved, Widespread, Dynamic mRNA Methylation Program in Yeast Meiosis. Cell 155:1409-1421. doi:10.1016/j.cell.2013.10.047

Schwartz S, Bernstein D a, Mumbach MR, Jovanovic M, Herbst RH, Leon-Ricardo BX, Engreitz JM, Guttman M, Satija R, Lander ES, Fink G, Regev A. 2014a. Transcriptome-wide Mapping Reveals Widespread Dynamic-Regulated Pseudouridylation of ncRNA and mRNA. Cell 159:148-162. doi:10.1016/j.cell.2014.08.028

Schwartz S, Mumbach MR, Jovanovic M, Wang T, Maciag K, Bushkin GG, Mertins P, Ter-Ovanesyan D, Habib N, Cacchiarelli D, Sanjana NE, Freinkman E, Pacold ME, Satija R, Mikkelsen TS, Hacohen N, Zhang F, Carr SA, Lander ES, Regev A. 2014b. Perturbation of m6A writers reveals two distinct classes of mRNA methylation at internal and 5' sites. Cell Rep 8:284-296. doi:10.1016/j.celrep.2014.05.048

Seila AC, Calabrese JM, Levine SS, Yeo GW, Rahl PB, Flynn RA, Young RA, Sharp PA. 2008. Divergent transcription from active promoters. Science 322:18491851. doi:10.1126/science. 1162253

Semotok JL, Cooperstock RL, Pinder BD, Vari HK, Lipshitz HD, Smibert CA. 2005. Smaug recruits the CCR4/POP2/NOT deadenylase complex to trigger maternal 
transcript localization in the early Drosophila embryo. Curr Biol 15:284-294. doi:10.1016/j.cub.2005.01.048

Shaheen R, Tasak M, Maddirevula S, Abdel-Salam GMH, Sayed ISM, Alazami AM, Al-Sheddi T, Alobeid E, Phizicky EM, Alkuraya FS. 2019. PUS7 mutations impair pseudouridylation in humans and cause intellectual disability and microcephaly. Hum Genet. doi:10.1007/s00439-019-01980-3

Sharma S, Langhendries J, Watzinger P, Peter K, Entian K, Lafontaine DLJ. 2015. Yeast Kre33 and human NAT10 are conserved 18S rRNA cytosine acetyltransferases that modify tRNAs assisted by the adaptor Tan 1 / THUMPD1. Nucleic Acids Res 43:2242-2258. doi:10.1093/nar/gkv075

Shi H, Moore PB. 2000. The crystal structure of yeast phenylalanine tRNA at $1.93 \mathrm{~A}$ resolution: a classic structure revisited. RNA 6:1091-1105.

Smith AM, Jain M, Mulroney L, Geralde DR, Akeson M. 2017. Reading canonical and modified nucleotides in $16 \mathrm{~S}$ ribosomal RNA using nanopore direct RNA sequencing. bioRxiv.

Smith JE, Alvarez-Dominguez JR, Kline N, Huynh NJ, Geisler S, Hu W, Coller J, Baker KE. 2014. Translation of small open reading frames within unannotated RNA transcripts in Saccharomyces cerevisiae. Cell Rep 7:1858-1866. doi:10.1016/j.celrep.2014.05.023

Smith T, Heger A, Sudbery I. 2017. UMI-tools: modeling sequencing errors in Unique Molecular Identifiers to improve quantification accuracy. Genome Res 27:491499. doi:10.1101/gr.209601.116

Squires JE, Patel HR, Nousch M, Sibbritt T, Humphreys DT, Parker BJ, Suter CM, Preiss T. 2012. Widespread occurrence of 5-methylcytosine in human coding and non-coding RNA. Nucleic Acids Res 40:5023-5033. doi:10.1093/nar/gks144

Steinberg S, Cedergren R. 1995. A correlation between N2-dimethylguanosine presence and alternate tRNA conformers. RNA 1:886-891.

Steiner-Mosonyi M, Mangroo D. 2004. The nuclear tRNA aminoacylation-dependent pathway may be the principal route used to export tRNA from the nucleus in Saccharomyces cerevisiae. Biochem J 378:809-816. doi:10.1042/BJ20031306

Steinmetz EJ, Conrad NK, Brow DA, Corden JL. 2001. RNA-binding protein Nrd1 directs poly(A)-independent 3'-end formation of RNA polymerase II transcripts. Nature 413:327-331. doi:10.1038/35095090

Stevens A, Poole TL. 1995. 5'-exonuclease-2 of Saccharomyces cerevisiae. Purification and features of ribonuclease activity with comparison to 5'exonuclease-1. J Biol Chem 270:16063-16069. 
Sun M, Schwalb B, Schulz D, Pirkl N, Etzold S, Larivière L, Maier KC, Seizl M, Tresch A, Cramer P. 2012. Comparative dynamic transcriptome analysis (cDTA) reveals mutual feedback between mRNA synthesis and degradation. Genome Res 22:1350-1359. doi:10.1101/gr.130161.111

Synowsky SA, van Wijk M, Raijmakers R, Heck AJR. 2009. Comparative multiplexed mass spectrometric analyses of endogenously expressed yeast nuclear and cytoplasmic exosomes. J Mol Biol 385:1300-1313. doi:10.1016/j.jmb.2008.11.011

Tan-Wong SM, Zaugg JB, Camblong J, Xu Z, Zhang DW, Mischo HE, Ansari AZ, Luscombe NM, Steinmetz LM, Proudfoot NJ. 2012. Gene loops enhance transcriptional directionality. Science 338:671-675. doi:10.1126/science. 1224350

Tardu M, Lin Q, Koutmou KS. 2018. N4-acetylcytidine and 5-formylcytidine are present in Saccharomyces cerevisiae mRNAs. bioRxiv. doi:10.1101/327585

Tharun S, Parker R. 2001. Targeting an mRNA for decapping: displacement of translation factors and association of the Lsm1p-7p complex on deadenylated yeast mRNAs. Mol Cell 8:1075-1083.

Theler D, Dominguez C, Blatter M, Boudet J, Allain H, Allain FH-T. 2014. Solution structure of the YTH domain in complex with N6-methyladenosine RNA: a reader of methylated RNA. Nucleic Acids Res 42:13911-13919. doi:10.1093/nar/gku1116

Thiaville PC, Legendre R, Rojas-Benítez D, Baudin-Baillieu A, Hatin I, Chalancon G, Glavic A, Namy O, de Crécy-Lagard V. 2016. Global translational impacts of the loss of the tRNA modification t(6)A in yeast. Microb cell 3:29-45. doi:10.15698/mic2016.01.473

Thiebaut M, Kisseleva-Romanova E, Rougemaille M, Boulay J, Libri D. 2006. Transcription Termination and Nuclear Degradation of Cryptic Unstable Transcripts: A Role for the Nrd1-Nab3 Pathway in Genome Surveillance. Mol Cell 23:853-864. doi:10.1016/j.molcel.2006.07.029

Thompson DM, Parker R. 2007. Cytoplasmic decay of intergenic transcripts in Saccharomyces cerevisiae. Mol Cell Biol 27:92-101. doi:10.1128/MCB.0102306

Torrent M, Chalancon G, Groot NS De, Wuster A, Babu MM. 2018. Cells alter their tRNA abundance to selectively regulate protein synthesis during stress conditions. Sci Signal 6409:1-10.

Torres AG, Batlle E, Ribas de Pouplana L. 2014. Role of tRNA modifications in human diseases. Trends Mol Med 20:306-314. 
doi:10.1016/j.molmed.2014.01.008

Trewick SC, Henshaw TF, Hausinger RP, Lindahl T, Sedgwick B. 2002. Oxidative demethylation by Escherichia coli AlkB directly reverts DNA base damage. Nature. doi:10.1038/nature00908

Tuck AC, Tollervey D. 2013. A transcriptome-wide atlas of RNP composition reveals diverse classes of mRNAs and IncRNAs. Cell 154:996-1009. doi:10.1016/j.cell.2013.07.047

Tucker M, Valencia-Sanchez MA, Staples RR, Chen J, Denis CL, Parker R. 2001. The transcription factor associated Ccr4 and Caf1 proteins are components of the major cytoplasmic mRNA deadenylase in Saccharomyces cerevisiae. Cell 104:377-386. doi:10.1016/S0092-8674(01)00225-2

Tudek A, Porrua O, Kabzinski T, Lidschreiber M, Kubicek K, Fortova A, Lacroute F, Vanacova S, Cramer P, Stefl R, Libri D. 2014. Molecular basis for coordinating transcription termination with noncoding RNA degradation. Mol Cell 55:467481. doi:10.1016/j.molcel.2014.05.031

Turowski TW, Tollervey D. 2015. Cotranscriptional events in eukaryotic ribosome synthesis. Wiley Interdiscip Rev RNA 6:129-139. doi:10.1002/wrna.1263

Urbonavičius J, Qian Q, Durand JMB, Hagervall TG, Björk GR. 2001. Improvement of reading frame maintenance is a common function for several tRNA modifications. EMBO J 20:4863-4873. doi:10.1093/emboj/20.17.4863

Van Der Maaten LJP, Hinton GE. 2008. Visualizing high-dimensional data using tsne. J Mach Learn Res 9:2579-2605. doi:10.1007/s10479-011-0841-3

van Dijk EL, Chen CL, d'Aubenton-Carafa Y, Gourvennec S, Kwapisz M, Roche V, Bertrand C, Silvain M, Legoix-Ne P, Loeillet S, Nicolas A, Thermes C, Morillon A. 2011. XUTs are a class of Xrn1-sensitive antisense regulatory non-coding RNA in yeast. Nature 475:114-117. doi:10.1038/nature10118

van Hoof A, Frischmeyer PA, Dietz HC, Parker R. 2002. Exosome-mediated recognition and degradation of mRNAs lacking a termination codon. Science 295:2262-2264. doi:10.1126/science.1067272

Van Nostrand EL, Pratt GA, Shishkin AA, Gelboin-Burkhart C, Fang MY, Sundararaman B, Blue SM, Nguyen TB, Surka C, Elkins K, Stanton R, Rigo F, Guttman M, Yeo GW. 2016. Robust transcriptome-wide discovery of RNAbinding protein binding sites with enhanced CLIP (eCLIP). Nat Methods 13:508514. doi: $10.1038 / n m e t h .3810$

Vanacova S, Stefl R. 2007. The exosome and RNA quality control in the nucleus. EMBO Rep 8:651-657. doi:10.1038/sj.embor.7401005

Vasiljeva L, Buratowski S. 2006. Nrd1 interacts with the nuclear exosome for 3' 
processing of RNA polymerase II transcripts. Mol Cell 21:239-248.

doi:10.1016/j.molcel.2005.11.028

Waas WF, Druzina Z, Hanan M, Schimmel P. 2007. Role of a tRNA Base

Modification and Its Precursors in Frameshifting in Eukaryotes. J Biol Chem

282:26026-26034. doi:10.1074/jbc.M703391200

Wang L, Lewis MS, Johnson AW. 2005. Domain interactions within the Ski2/3/8 complex and between the Ski complex and Ski7p. RNA 11:1291-1302. doi:10.1261/rna.2060405

Wang X, Lu Z, Gomez A, Hon GC, Yue Y, Han D, Fu Y, Parisien M, Dai Q, Jia G, Ren B, Pan T, He C. 2014. N6-methyladenosine-dependent regulation of messenger RNA stability. Nature 505:117-120. doi:10.1038/nature12730

Wang X, Zhao BS, Roundtree IA, Lu Z, Han D, Ma H, Weng X, Chen K, Shi H, He C. 2015. N(6)-methyladenosine Modulates Messenger RNA Translation Efficiency. Cell 161:1388-1399. doi:10.1016/j.cell.2015.05.014

Wang Y, Li Y, Toth JI, Petroski MD, Zhang Z, Zhao JC. 2014. N6-methyladenosine modification destabilizes developmental regulators in embryonic stem cells. Nat Cell Biol 16:191-198. doi:10.1038/ncb2902

Warda AS, Kretschmer J, Hackert P, Lenz C, Urlaub H, Höbartner C, Sloan KE, Bohnsack MT. 2017. Human METTL16 is a N6-methyladenosine (m6A) methyltransferase that targets pre-mRNAs and various non-coding RNAs 18:2004-2014. doi:10.15252/embr.201744940

Webster MW, Chen Y, Stowell JAW, Graveley BR, Coller J, Passmore LA, Webster MW, Chen Y, Stowell JAW, Alhusaini N, Sweet T, Graveley BR. 2018. mRNA Deadenylation Is Coupled to Translation Rates by the Differential Activities of Ccr4-Not Nucleases. Mol Cell 70:1089-1100.e8. doi:10.1016/j.molcel.2018.05.033

Weixlbaumer A, Murphy F V, Dziergowska A, Malkiewicz A, Vendeix FAP, Agris PF, Ramakrishnan V. 2007. Mechanism of expanding the decoding capacity of tRNAs by modification of uridines. Nat Struct Mol Biol 14:498-502. doi:10.1038/nsmb1242

Wells SE, Hillner PE, Vale RD, Sachs AB. 1998. Circularization of mRNA by eukaryotic translation initiation factors. Mol Cell 2:135-140. doi:10.1016/S10972765(00)80122-7

Whitelaw E, Proudfoot N. 1986. Alpha-thalassaemia caused by a poly(A) site mutation reveals that transcriptional termination is linked to $3^{\prime}$ end processing in the human alpha 2 globin gene. EMBO J 5:2915-2922.

Wolf J, Passmore LA. 2014. Deadenylation by Pan2 / Pan3. Biochem Soc Trans 
42:184-187. doi:10.1042/BST20130211.mRNA

Wyers F, Rougemaille M, Badis G, Rousselle JC, Dufour ME, Boulay J, Régnault B, Devaux F, Namane A, Séraphin B, Libri D, Jacquier A. 2005. Cryptic Pol II transcripts are degraded by a nuclear quality control pathway involving a new poly(A) polymerase. Cell 121:725-737. doi:10.1016/j.cell.2005.04.030

Xiao W, Adhikari S, Xiao W, Adhikari S, Dahal U, Chen Y, Hao Y, Sun B, Sun H. 2016. Nuclear m6A Reader YTHDC1 Regulates mRNA Splicing. Mol Cell 61:507-519. doi:10.1016/j.molcel.2016.01.012

Xiong X, Li X, Wang KUN, Yi C. 2018. Perspectives on topology of the human $\mathrm{m} 1 \mathrm{~A}$ methylome at single nucleotide resolution. RNA 24:1437-1442. doi:10.1261/rna.067694.118.5

Xu C, Liu K, Ahmed H, Loppnau P, Schapira M, Min J. 2015. Structural Basis for the Discriminative Recognition of N6-Methyladenosine RNA by the Human YT521B Homology Domain Family of Proteins. J Biol Chem 290:24902-24913. doi:10.1074/jbc.M115.680389

Xu L, Liu X, Sheng N, Oo KS, Liang J, Chionh YH, Xu J, Ye F. 2017. Three distinct 3-methylcytidine (m3C) methyltransferases modify tRNA and mRNA in mice and humans. J Biol Chem 292:14695-14703. doi:10.1074/jbc.M117.798298

Xu Z, Wei W, Gagneur J, Perocchi F, Clauder-Munster S, Camblong J, Guffanti E, Stutz F, Huber W, Steinmetz LM. 2009. Bidirectional promoters generate pervasive transcription in yeast. Nature 457:1033-1037. doi:10.1038/nature07728

Yacoubi B El, Hatin I, Deutsch C, Kahveci T, Rousset J, Iwata-reuyl D. 2011. A role for the universal Kae1/Qri7/YgjD (COG0533) family in tRNA modification 30:882-893. doi:10.1038/emboj.2010.363

Yoshihisa T, Yunoki-Esaki K, Ohshima C, Tanaka N, Endo T. 2003. Possibility of cytoplasmic pre-tRNA splicing: the yeast tRNA splicing endonuclease mainly localizes on the mitochondria. Mol Biol Cell 14:3266-3279. doi:10.1091/mbc.e02-11-0757

Yudkovsky N, Ranish JA, Hahn S. 2000. A transcription reinitiation intermediate that is stabilized by activator. Nature 408:225-229. doi:10.1038/35041603

Zaringhalam M, Papavasiliou FN. 2016. Pseudouridylation meets next-generation sequencing. Methods 107:63-72. doi:10.1016/j.ymeth.2016.03.001

Zhang Z, Theler D, Kaminska KH, Hiller M, de la Grange P, Pudimat R, Rafalska I, Heinrich B, Bujnicki JM, Allain FH-T, Stamm S. 2010. The YTH domain is a novel RNA binding domain. J Biol Chem 285:14701-14710.

doi:10.1074/jbc.M110.104711 
Zhao Y, Majid MC, Soll JM, Brickner JR, Dango S, Mosammaparast N. 2015. Noncanonical regulation of alkylation damage resistance by the OTUD4 deubiquitinase. EMBO J 34:1687-1703. doi:10.15252/embj.201490497

Zheng G, Dahl JA, Niu Y, Fedorcsak P, Huang C-M, Li CJ, Vagbo CB, Shi Y, Wang W-L, Song S-H, Lu Z, Bosmans RPG, Dai Q, Hao Y-J, Yang X, Zhao W-M, Tong W-M, Wang X-J, Bogdan F, Furu K, Fu Y, Jia G, Zhao X, Liu J, Krokan HE, Klungland A, Yang Y-G, He C. 2013. ALKBH5 is a mammalian RNA demethylase that impacts RNA metabolism and mouse fertility. Mol Cell 49:1829. doi:10.1016/j.molcel.2012.10.015

Zhu T, Roundtree IA, Wang P, Wang X, Wang L, Sun C, Tian Y, Li J, He C, Xu Y. 2014. Crystal structure of the YTH domain of YTHDF2 reveals mechanism for recognition of N6-methyladenosine. Cell Res. doi:10.1038/cr.2014.152

Zinder JC, Lima CD. 2017. Targeting RNA for processing or destruction by the eukaryotic RNA exosome and its cofactors. Genes Dev 31:88-100. doi:10.1101/gad.294769.116 
Bibliography

- 140 - 


\section{Appendix}

\subsection{Supplementary Information}

\subsubsection{Figures}

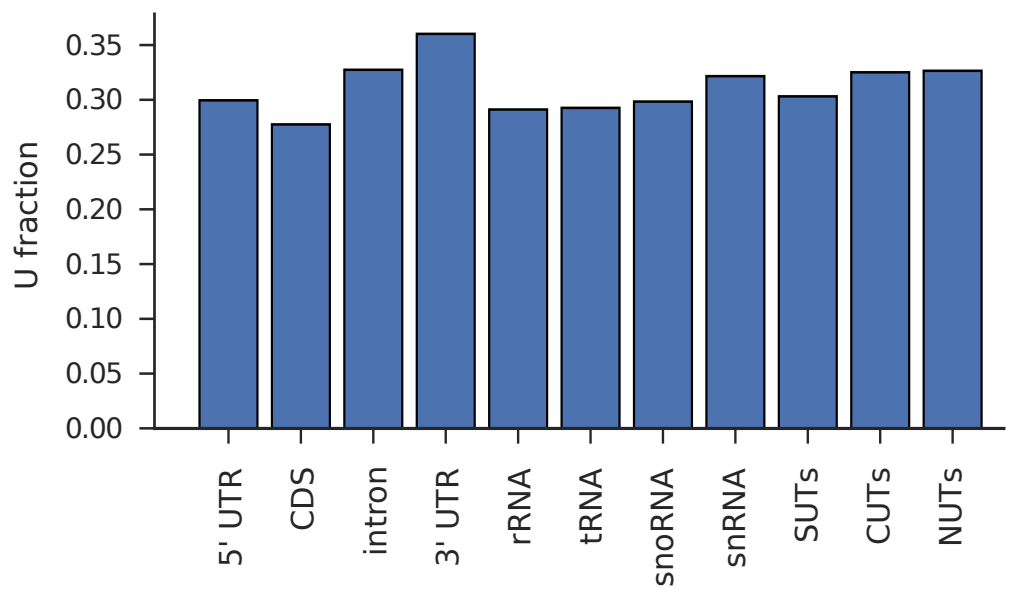

Figure 28: Different transcript classes have comparable U-content.

Fraction of $U$ over all bases in transcript classes studied in Figure 8 (untranslated region (UTR); intron; coding sequence (CDS), ribosomal RNA (rRNA), transfer RNA (tRNA), small nucleolar RNA (snoRNA), small nuclear RNA (snRNA), stable unannotated transcripts (SUTs), cryptic unstable transcripts (CUTs), Nrd1unterminated transcripts (NUTs)). 


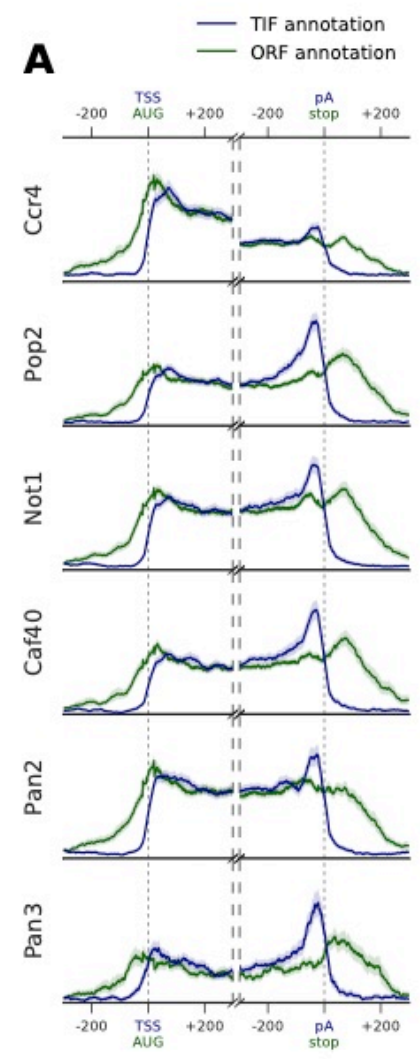

B

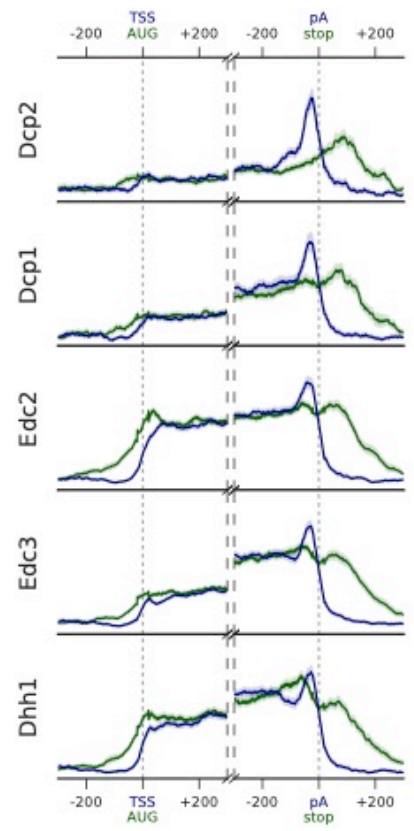

C

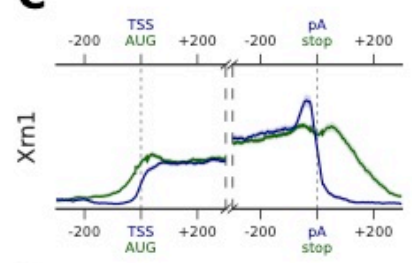

$\mathbf{F}$

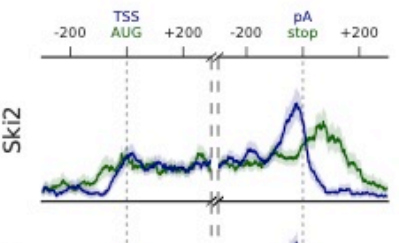

$\stackrel{m}{\text { 竞 }}$

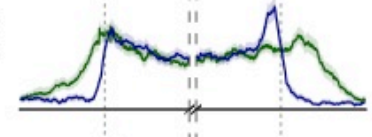

窝

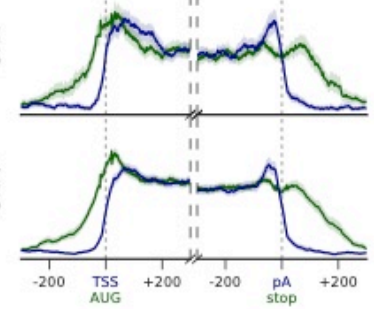

D

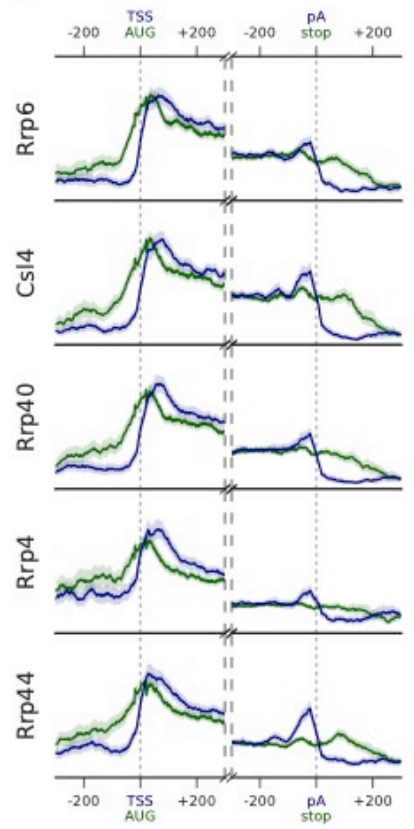

$\mathbf{E}$

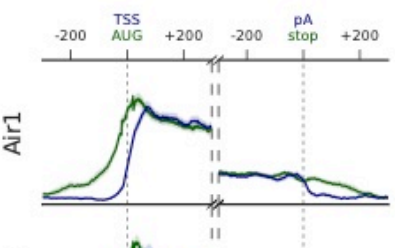

虽

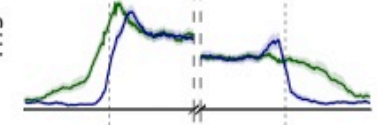

竞

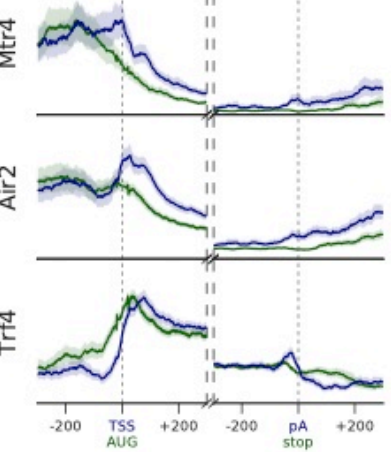

G

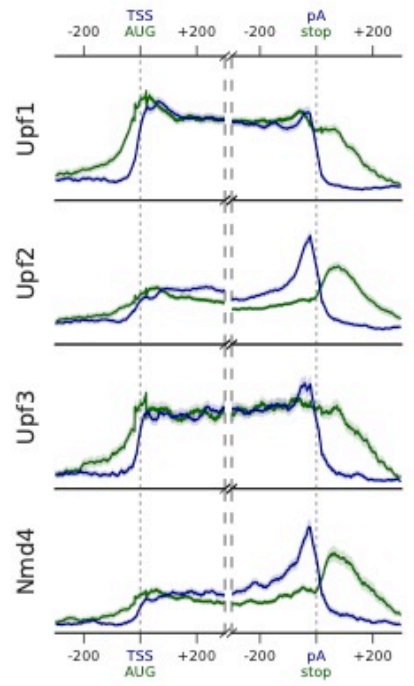

Figure 29: Metagene profiles of yeast RNA degradation factors centered on translation start and stop sites in comparison to TIF-annotated TSS and pA sites.

Transcript-averaged PAR-CLIP occupancy profiles is shown for RNA degradation factors involved in A) deadenylation, B) decapping, C) $5^{\prime} \rightarrow 3^{\prime}$ exonuclease Xrn1, D) 
exosome, E) TRAMP, F) Ski, and G) NMD. Transcripts are aligned either at transcript start site (TSS) and poly-adenylation ( $\mathrm{pA}$ ) site (marked with blue) or at their start and stop codons (marked with green). TIF-seq based annotation is shown in blue $(n=3,193$ for TSS and pA site profiles) (Pelechano et al., 2013). Open reading frames (ORF) annotated in the SGD (version 64.2.1) are shown in green ( $n=4,012$ for TSS, and $\mathrm{n}=3,965$ for $\mathrm{pA}$ site selected transcripts). To avoid contaminating signals from neighboring genes, we filtered out regions that had annotations upstream and downstream of the centered gene (up to $700 \mathrm{nt}$ ) (Methods). Shaded areas (in blue TIFseq annotation, or in green for ORF annotation) depict $95 \%$ confidence intervals derived from bootstrapping genes. Comparison between these two profiles highlights preferences for end binding degradation factors in binding to untranslated regions at the two sides of the transcript.
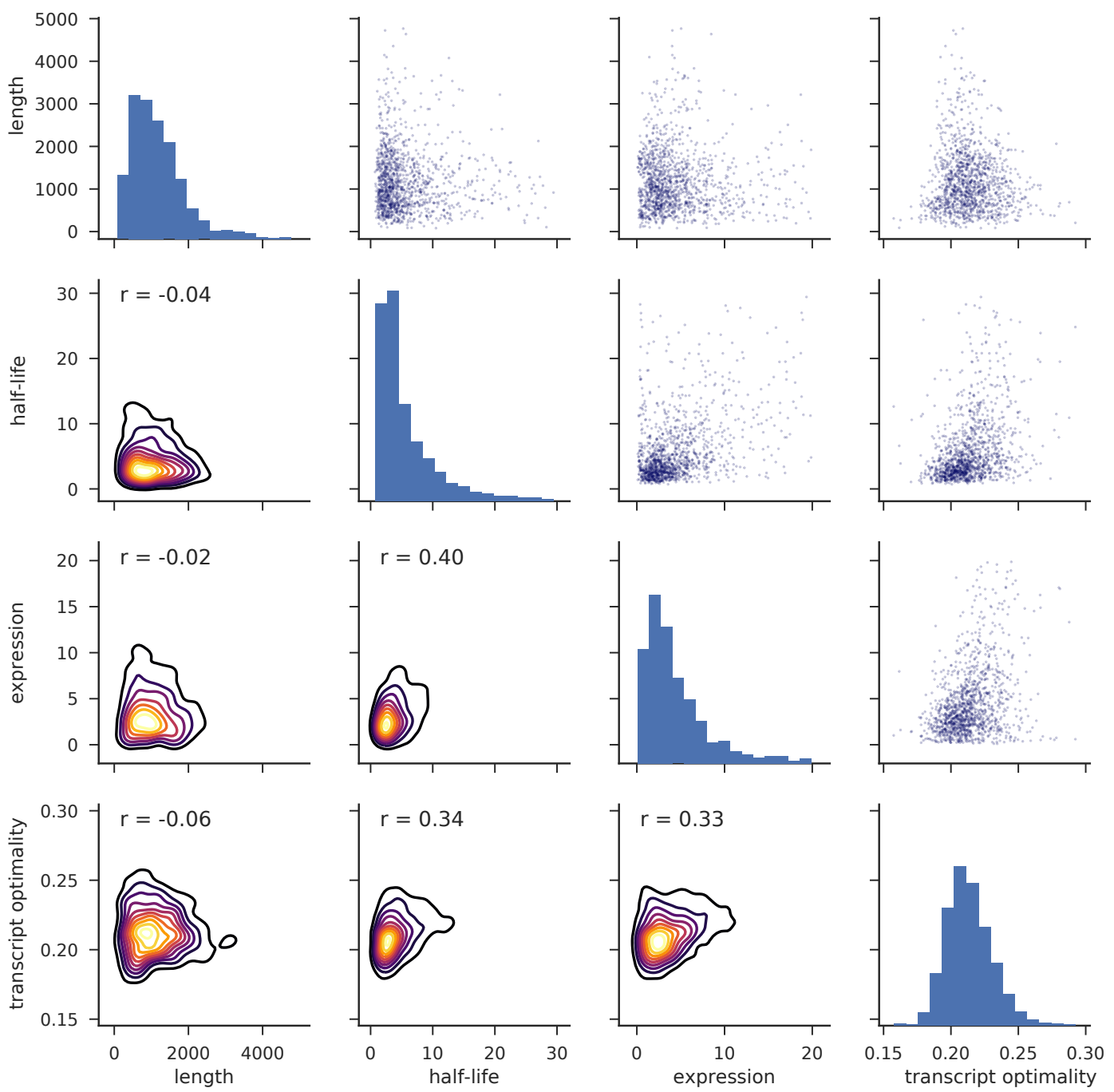

Figure 30: Distributions of transcript length, half-life, expression level and transcript optimality for yeast $m R N A$ s.

Histograms on the diagonal show distributions of length, half-life (Methods), expression level (Baejen et al., 2017) and transcript optimality (Pechmann and 
Frydman, 2013). Pairwise comparisons of features are shown as scatter plots (top right) and kernel density estimates (KDEs) of bivariate densities are shown in the bottom with Pearson correlation values ( $r$ ) (Methods).

A

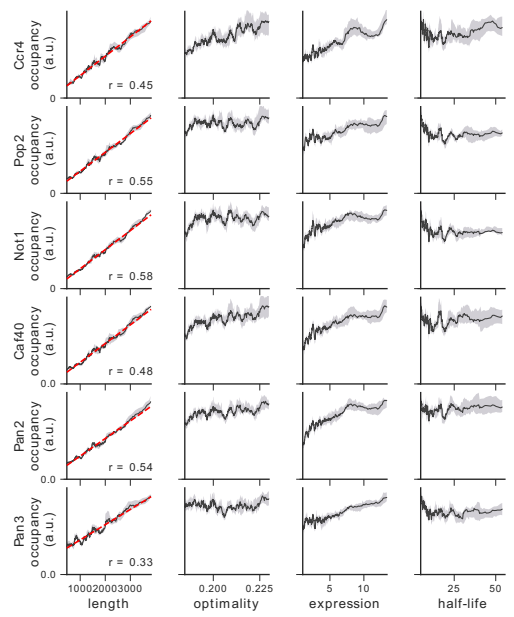

B
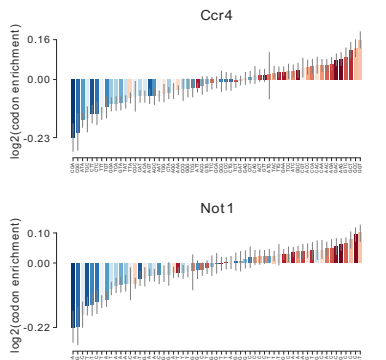

Pan2

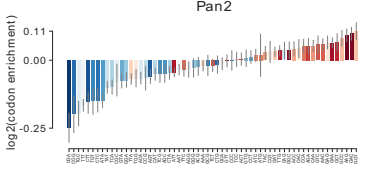

61 codons (sorted by enrichment)

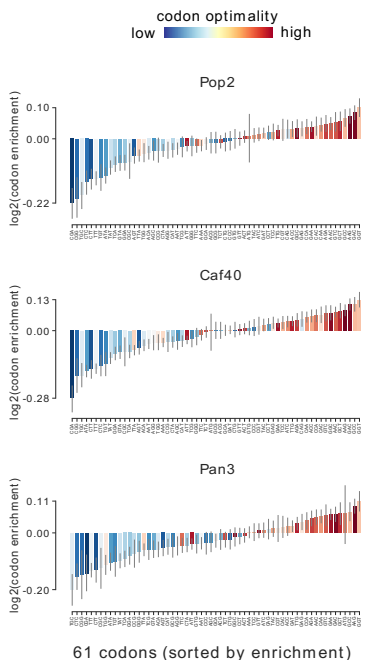

61 codons (sorted by enrichment)

Figure 31: Occupancies of deadenylation factors (Ccr4, Pop2, Not1, Caf40, Pan2, and Pan3) compared to transcript length, optimality, expression level, and half-life. A) To understand binding specificity of deadenylation factors, the total occupancy of each factor on a transcript is plotted against various transcript features (Grey shading: $95 \%$ confidence intervals generated by bootstrapping transcripts). B) Same analysis as in Figure 12B: Codon enrichment shows deviations in codon frequencies of transcripts bound by a degradation factor compared to each codon's frequency on all coding sequences. Each bar is colored according to its codon-optimality with highly optimal codons in dark red and highly non-optimal codons in dark blue. (Grey lines: $90 \%$ confidence intervals generated by bootstrapping coding sequences).

A

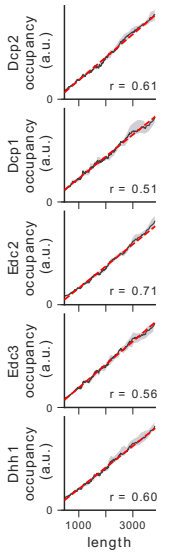

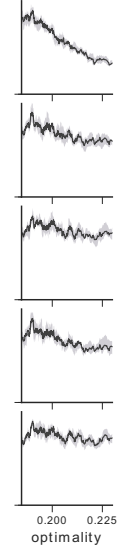

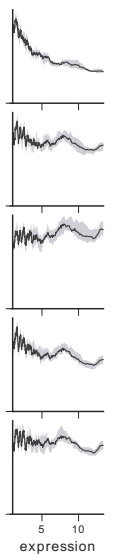

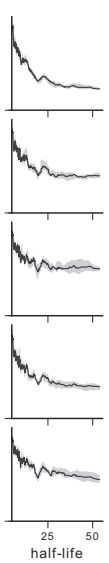

B

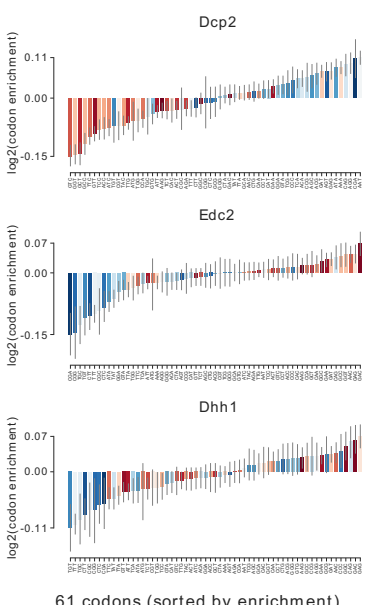

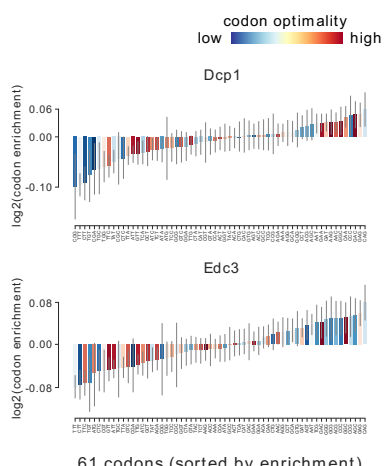

61 codons (sorted by enrichment)

Figure 32: Occupancies of decapping factors (Dcp2, Dcp1, Edc2, Edc3, and Dhh1) compared to transcript length, optimality, expression level, and half-life. 
A) To understand binding specificity of decapping factors, the total occupancy of each factor on a transcript is plotted against various transcript features (Grey shading: 95\% confidence intervals generated by bootstrapping transcripts). B) Same analysis as in Figure 12B: Codon enrichment shows deviations in codon frequencies of transcripts bound by a degradation factor compared to each codon's frequency on all coding sequences. Each bar is colored according to its codon-optimality with highly optimal codons in dark red and highly non-optimal codons in dark blue. (Grey lines: 90\% confidence intervals generated by bootstrapping coding sequences).

A

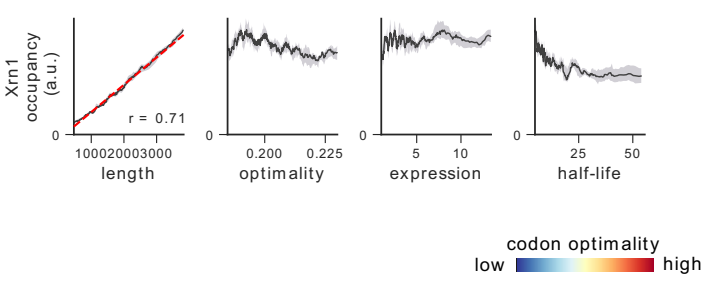

B

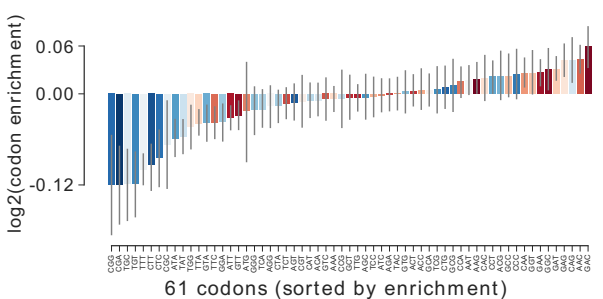

Figure 33: Occupancy of Xrn1 compared to transcript length, optimality, expression level, and half-life.

A) To understand binding specificity of Xrn1 on various mRNAs, the total occupancy of Xrn1 on a transcript is plotted against various transcript features (Grey shading: 95\% confidence intervals generated by bootstrapping transcripts). B) Same analysis as in Figure 12B: Codon enrichment shows deviations in codon frequencies of transcripts bound by a degradation factor compared to each codon's frequency on all coding sequences. Each bar is colored according to its codon-optimality with highly optimal codons in dark red and highly non-optimal codons in dark blue. (Grey lines: 90\% confidence intervals generated by bootstrapping coding sequences). 


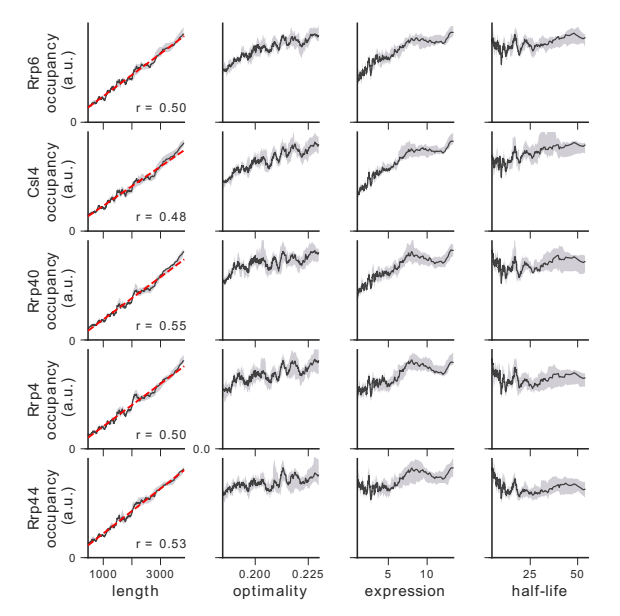

B

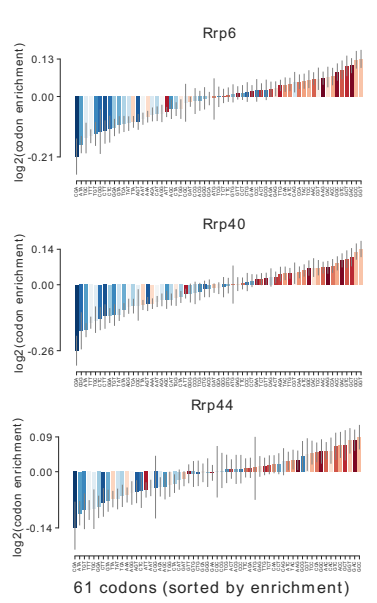

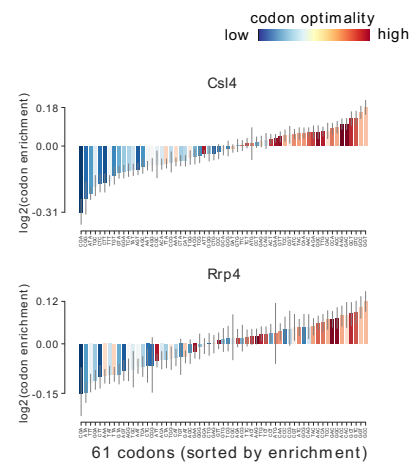

61 codons (sorted by enrichment)

Figure 34: Occupancies of exosome components (Rrp6, Cs/4, Rrp40, Rrp4, and Rrp44) compared to transcript length, optimality, expression level, and half-life.

A) To understand binding specificity of exosome components, the total occupancy of each factor on a transcript is plotted against various transcript features (Grey shading: $95 \%$ confidence intervals generated by bootstrapping transcripts). B) Same analysis as in Figure 12B: Codon enrichment shows deviations in codon frequencies of transcripts bound by a degradation factor compared to each codon's frequency on all coding sequences. Each bar is colored according to its codon-optimality with highly optimal codons in dark red and highly non-optimal codons in dark blue. (Grey lines: $90 \%$ confidence intervals generated by bootstrapping coding sequences).

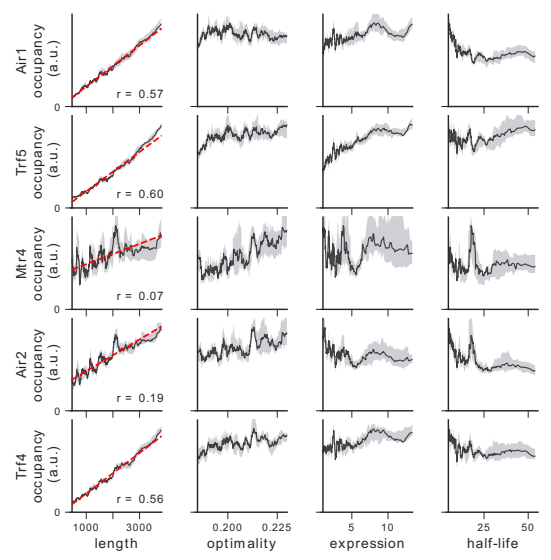

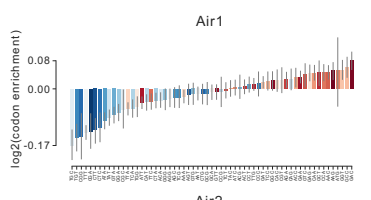

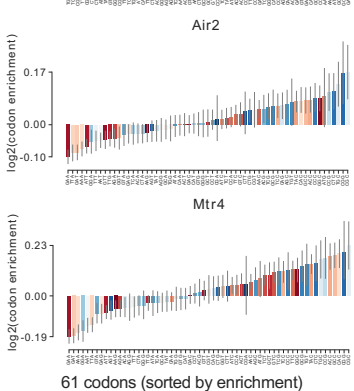

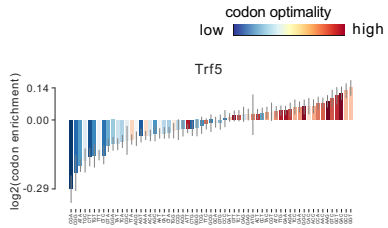

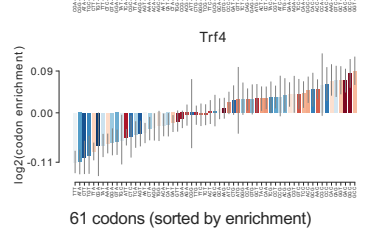

Figure 35: Occupancies for components of the TRAMP complex (Air1, Trf5, Mtr4, Air2, and Trf4) compared to transcript length, optimality, expression level, and halflife.

A) To understand binding specificity of TRAMP components, the total occupancy of each factor on a transcript is plotted against various transcript features (Grey shading: 95\% confidence intervals generated by bootstrapping transcripts). B) Same analysis as in Figure 5B: Codon enrichment shows deviations in codon frequencies of 
transcripts bound by a degradation factor compared to each codon's frequency on all coding sequences. Each bar is colored according to its codon-optimality with highly optimal codons in dark red and highly non-optimal codons in dark blue. (Grey lines: $90 \%$ confidence intervals generated by bootstrapping coding sequences).

A

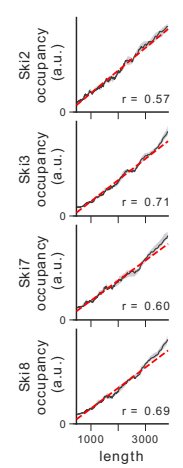

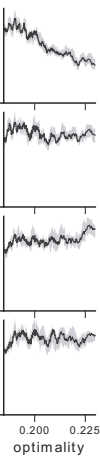
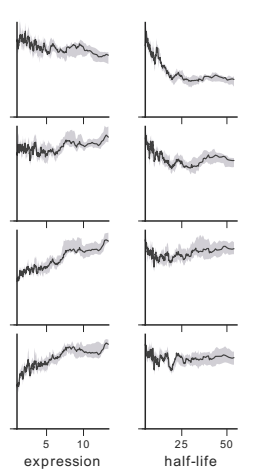

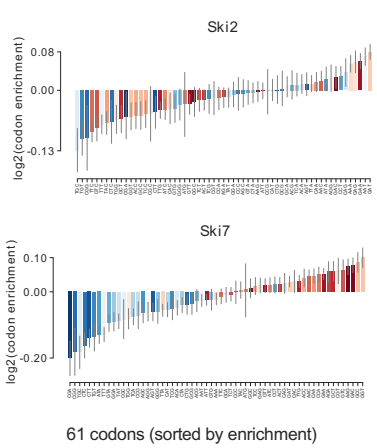

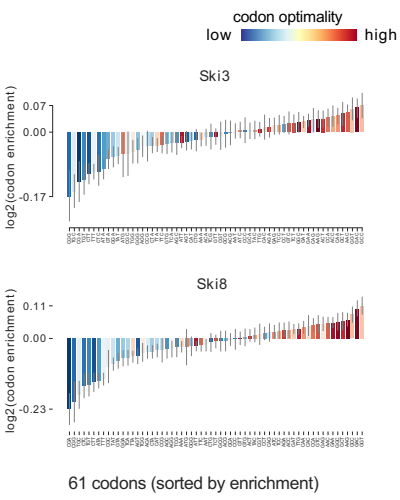

Figure 36: Occupancies for components of the Ski complex (Ski2, Ski3, Ski7, and Ski8) compared to transcript length, optimality, expression level, and half-life.

A) To understand binding specificity of factors in the Ski complex, the total occupancy of each factor on a transcript is plotted against various transcript features (Grey shading: $95 \%$ confidence intervals generated by bootstrapping transcripts). B) Same analysis as in Figure 12B: Codon enrichment shows deviations in codon frequencies of transcripts bound by a degradation factor compared to each codon's frequency on all coding sequences. Each bar is colored according to its codon-optimality with highly optimal codons in dark red and highly non-optimal codons in dark blue. (Grey lines: $90 \%$ confidence intervals generated by bootstrapping coding sequences).

A

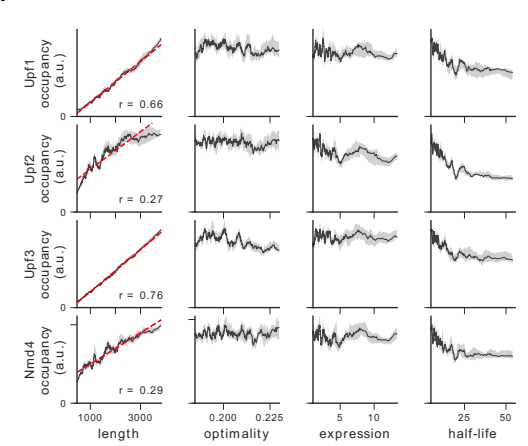

B

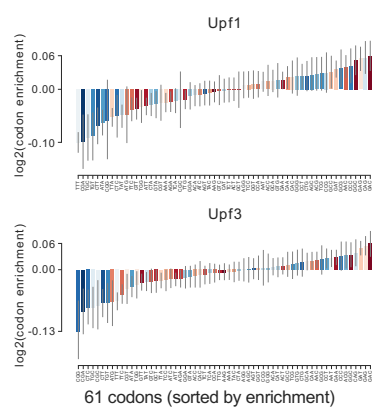

codon optimality high

Upf2

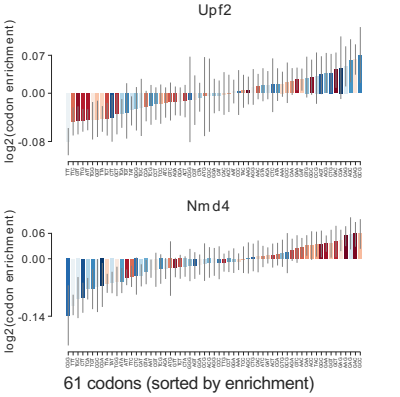

Figure 37: Occupancies for components of the NMD pathway (Upf1, Upf2, Upf3, and Nmd4) compared to transcript length, optimality, expression level, and half-life.

(A) To understand binding specificity of factors in the NMD pathway, the total occupancy of each factor on a transcript is plotted against various transcript features (Grey shading: 95\% confidence intervals generated by bootstrapping transcripts). (B) Same analysis as in Figure 12B: Codon enrichment shows deviations in codon 
frequencies of transcripts bound by a degradation factor compared to each codon's frequency on all coding sequences. Each bar is colored according to its codonoptimality with highly optimal codons in dark red and highly non-optimal codons in dark blue. (Grey lines: 90\% confidence intervals generated by bootstrapping coding sequences).

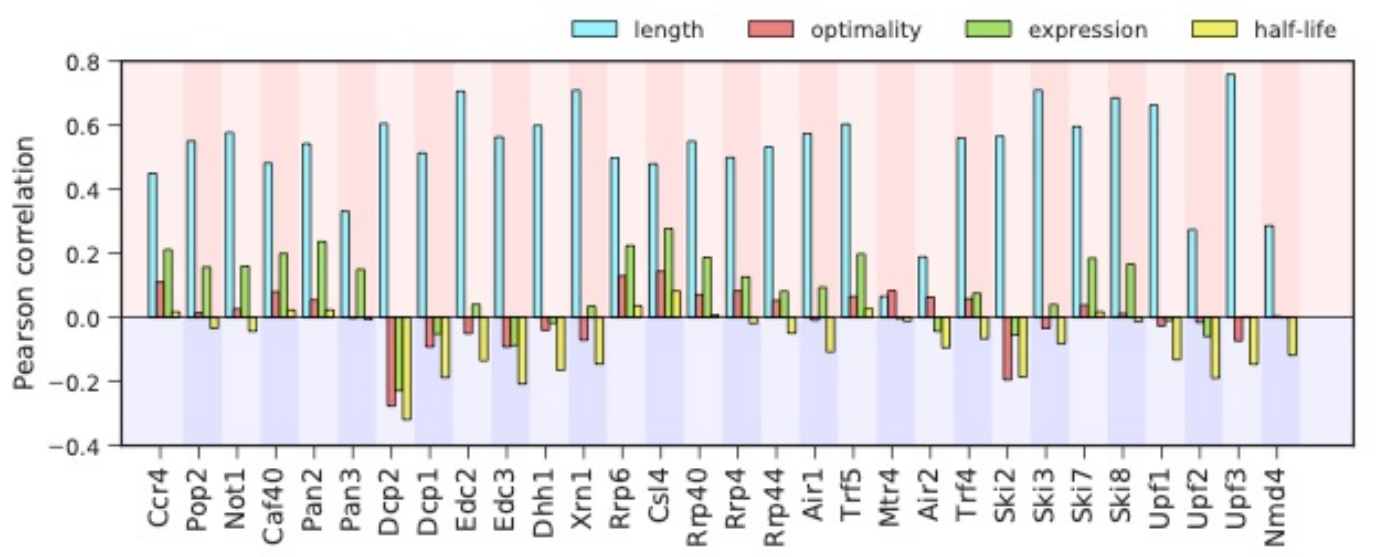

Figure 38: Correlation between binding to degradation factors and transcript length, codon-optimality, expression, and half-life.

Pearson correlation values between the binding strength of degradation factors (total occupancy over each transcript) and transcript length, transcript optimality (Pechmann and Frydman, 2013), expression level (Baejen et al., 2017), and half-life derived by multivariate linear regression analysis (Methods). 


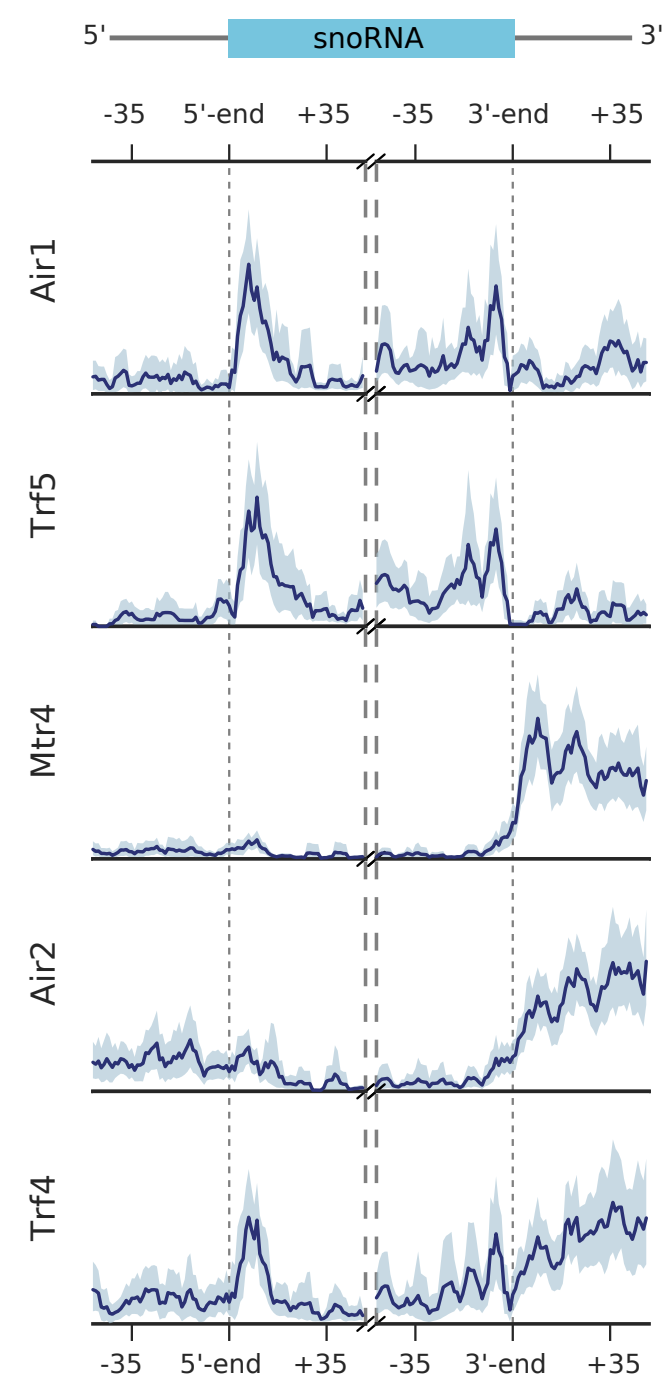

Figure 39: Metagene profiles for subunits of the TRAMP complex on snoRNA genes. Transcript averaged PAR-CLIP occupancy profiles are shown for Air1, Trf5, Mtr4, Air2, and Trf4. snoRNA genes are aligned either at their $5^{\prime}$ end or at their $3^{\prime}$ end $(n=77)$. Occupancy profiles are shown over the range of $\pm 35 \mathrm{nt}$. 
A

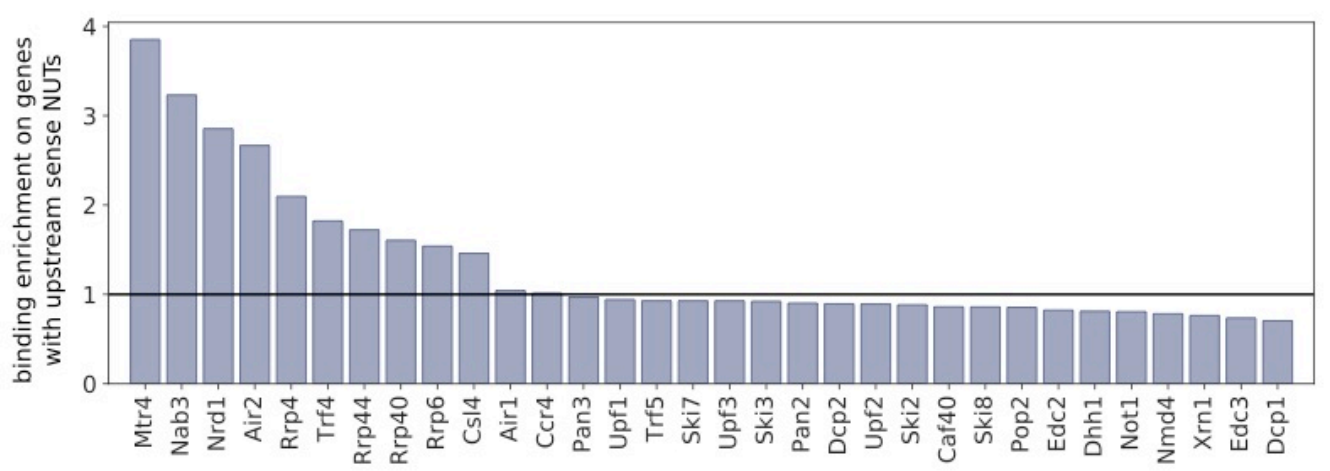

B
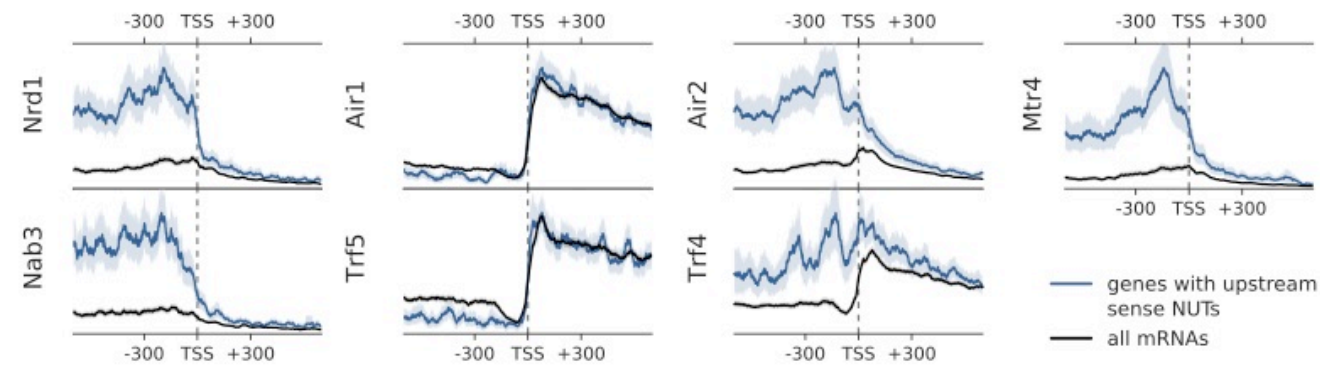

Figure 40: Comparison of binding profiles on genes containing annotated upstream sense NUTs with all mRNAs.

A) Binding enrichment of degradation factors around the TSS of genes with an upstream sense NUT. Enrichment is defined as the ratio of the average occupancy in the interval $[ \pm 300 \mathrm{nt}]$ of the TSS on these genes that contain an upstream NUT $(n=459)$ (Schulz et al., 2013) divided by the average occupancy on all genes. B) Transcriptaveraged PAR-CLIP occupancy profiles for all mRNAs (black) is compared to patterns derived from genes with upstream sense NUTs (blue). Transcripts were aligned at their TSS and averaged over the interval of [ $\pm 600 \mathrm{nt}]$. We compared Nrd1 and Nab3 profiles, known to process NUTs, with subunits of the TRAMP complex. 95\% confidence intervals obtained from bootstrapping genes are shown with grey and blue shades. 


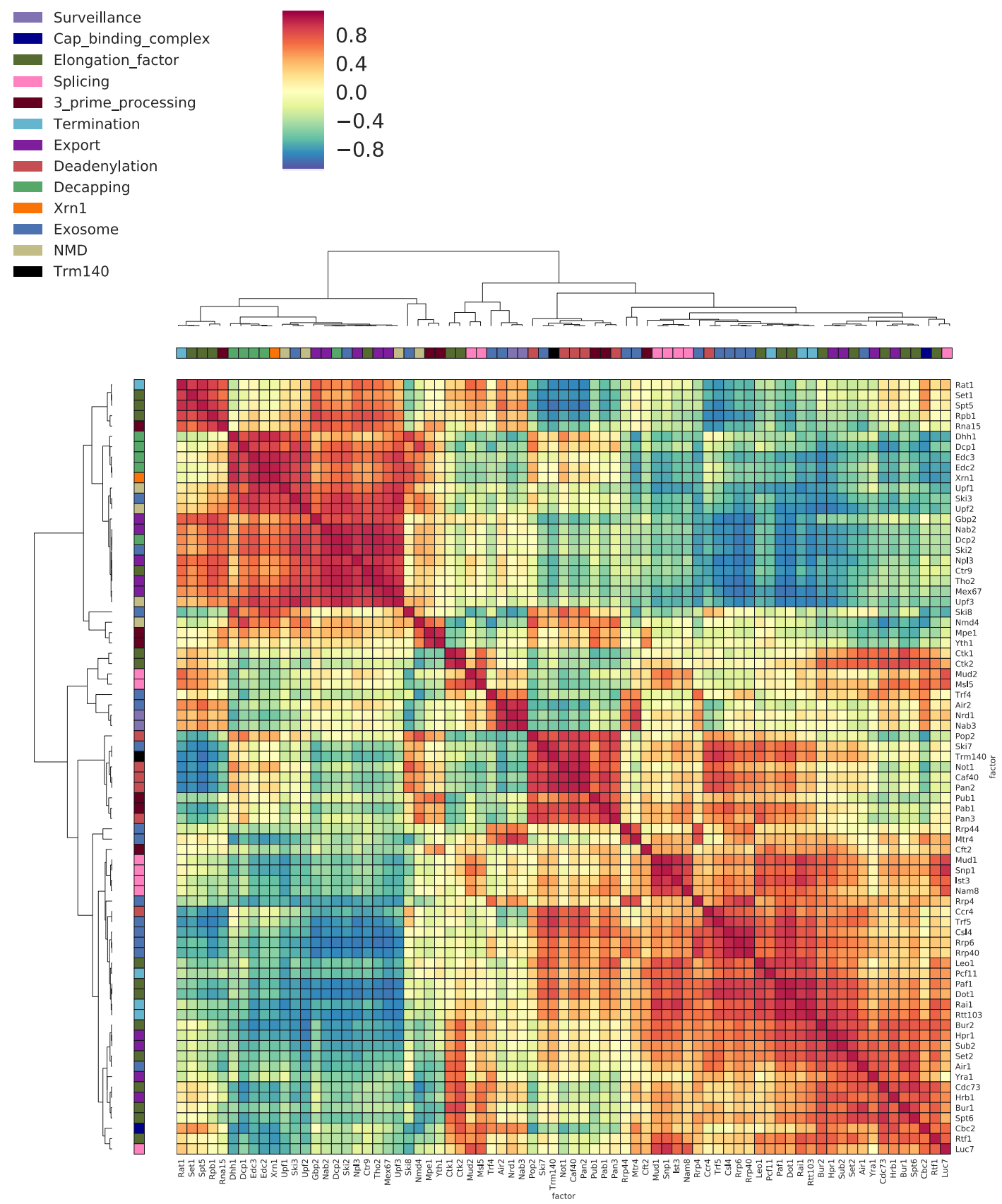

Figure 41: Co-localization coefficients for 75 RNA binding factors.

Pairwise correlation between normalized co-localization profiles of factors in a window of $40 \mathrm{nt}$ centered at PAR-CLIP cross-link sites is shown here. Analysis for 75 RNA binding factors, including Trm140, 30 factors from the RNA degradation study, and 44 factors from previous studies (Baejen et al., 2017, 2014; Battaglia et al., 2017; Schulz et al., 2013). High co-localization represents binding to the same position on transcripts (marked with dark red). Factors are clustered and color coded (left and upper border) according to their general function. Trm140 is colored in black and can be found in the central cluster together with deadenylation factors. 
7.1.2 Tables

Table 13: Overview of RNA processing factors and their respective published PARCLIP experiments.

\begin{tabular}{|c|c|c|c|c|c|}
\hline Pathway & Factor & Details & $\begin{array}{l}\text { High confidence } \\
\text { cross-link sites }\end{array}$ & Source & Accession number \\
\hline \multirow{2}{*}{ Surveillance } & Nrd1 & \multirow{2}{*}{ Nrd1/Nab3 complex } & 187164 & Schulz et al., 2013 & $\begin{array}{l}\text { ArrayExpress: } \\
\text { E-MTAB-1766 }\end{array}$ \\
\hline & Nab3 & & 91762 & Schulz et al., 2013 & $\begin{array}{l}\text { ArrayExpress: } \\
\text { E-MTAB-1766 }\end{array}$ \\
\hline $\begin{array}{l}\text { Cap binding } \\
\text { complex }\end{array}$ & $\mathrm{Cbc} 2$ & & 95530 & Baejen et al., 2014 & GEO: GSE59676 \\
\hline \multirow{15}{*}{ Elongation } & Bur1 & \multirow{2}{*}{ BUR kinase complex } & 232237 & & GEO: GSE81822 \\
\hline & Bur2 & & 169544 & Battaglia et al., 2017 & GEO: GSE81822 \\
\hline & Ctk1 & \multirow{2}{*}{ CTDK-I coomplex } & 257668 & Battaglia et al., 2017 & GEO: GSE81822 \\
\hline & Ctk2 & & 209835 & Battaglia et al., 2017 & GEO: GSE81822 \\
\hline & Cdc73 & \multirow{5}{*}{ PAF1 complex } & 234224 & Battaglia et al., 2017 & GEO: GSE81822 \\
\hline & Ctr9 & & 5361 & Battaglia et al., 2017 & GEO: GSE81822 \\
\hline & Leo1 & & 87202 & Battaglia et al., 2017 & GEO: GSE81822 \\
\hline & Paf1 & & 72871 & Battaglia et al., 2017 & GEO: GSE81822 \\
\hline & Rtf1 & & 52570 & Battaglia et al., 2017 & GEO: GSE81822 \\
\hline & Set1 & \multirow{3}{*}{$\begin{array}{l}\text { Histone methyl } \\
\text { transferase }\end{array}$} & 321575 & Battaglia et al., 2017 & GEO: GSE81822 \\
\hline & Set2 & & 90276 & Battaglia et al., 2017 & GEO: GSE81822 \\
\hline & Dot1 & & 88966 & Battaglia et al., 2017 & GEO: GSE81822 \\
\hline & Spt5 & DSIF & 193823 & Baejen et al. ,2017 & GEO: GSE79222 \\
\hline & Spt6 & Nucleosome remodeling & 204781 & Battaglia et al., 2017 & GEO: GSE81822 \\
\hline & Rpb1 & RNA polymerase & 153593 & Baejen et al. ,2017 & GEO: GSE79222 \\
\hline \multirow{7}{*}{ Splicing } & Ist3 & U2 snRNP & 3899 & Baejen et al., 2014 & GEO: GSE59676 \\
\hline & Nam8 & \multirow{4}{*}{ U1 snRNP } & 7097 & Baejen et al., 2014 & GEO: GSE59676 \\
\hline & Mud1 & & 8512 & Baejen et al., 2014 & GEO: GSE59676 \\
\hline & Snp1 & & 6773 & Baejen et al., 2014 & GEO: GSE59676 \\
\hline & Luc7 & & 33471 & Baejen et al., 2014 & GEO: GSE59676 \\
\hline & Mud2 & \multirow{2}{*}{ BBP/ U2AF65 } & 76190 & Baejen et al., 2014 & GEO: GSE59676 \\
\hline & Msl5 & & 81742 & Baejen et al., 2014 & GEO: GSE59676 \\
\hline \multirow{6}{*}{ 3' processing } & Pab1 & Poly(A) & 33422 & Baejen et al., 2014 & GEO: GSE59676 \\
\hline & Pub1 & Poly(U) & 72015 & Baejen et al., 2014 & GEO: GSE59676 \\
\hline & Rna15 & CFIA & 92792 & Baejen et al., 2014 & GEO: GSE59676 \\
\hline & Mpe1 & \multirow{3}{*}{ CPF } & 3743 & Baejen et al., 2014 & GEO: GSE59676 \\
\hline & Cft2 & & 32758 & Baejen et al., 2014 & GEO: GSE59676 \\
\hline & Yth1 & & 6506 & Baejen et al., 2014 & GEO: GSE59676 \\
\hline \multirow{4}{*}{ Termination } & Rat1 & Exoribonuclease & 122259 & Baejen et al. ,2017 & GEO: GSE79222 \\
\hline & Rai1 & & 23092 & Baejen et al. ,2017 & GEO: GSE79222 \\
\hline & Rtt103 & & 51954 & Baejen et al. ,2017 & GEO: GSE79222 \\
\hline & Pcf11 & & 1432 & Baejen et al. ,2017 & GEO: GSE79222 \\
\hline \multirow{9}{*}{ Export } & Hpr1 & \multirow{2}{*}{ THO } & 24616 & Baejen et al., 2014 & GEO: GSE59676 \\
\hline & Tho2 & & 3282 & Baejen et al., 2014 & GEO: GSE59676 \\
\hline & Gbp2 & \multirow{4}{*}{ TREX } & 151941 & Baejen et al., 2014 & GEO: GSE59676 \\
\hline & Hrb1 & & 132503 & Baejen et al., 2014 & GEO: GSE59676 \\
\hline & Mex67 & & 10917 & Baejen et al., 2014 & GEO: GSE59676 \\
\hline & Sub2 & & 39981 & Baejen et al., 2014 & GEO: GSE59676 \\
\hline & Yra1 & \multirow{3}{*}{ Adaptors } & 46438 & Baejen et al., 2014 & GEO: GSE59676 \\
\hline & Nab2 & & 99934 & Baejen et al., 2014 & GEO: GSE59676 \\
\hline & $\mathrm{Npl3}$ & & 131428 & Baejen et al., 2014 & GEO: GSE59676 \\
\hline
\end{tabular}


Table 14: High confidence m3C modification sites.

The $\mathrm{m} 3 \mathrm{C}$ modification sites including position (chr and position), number of $\mathrm{C} \rightarrow \mathrm{T}$ reads, unchanged reads (reference), the ratio (of transitions and reference reads), the assigned feature and gene name if annotated are shown. The $\mathrm{m} 3 \mathrm{C}$ modification sites were called using the Trm140 PAR-CLIP sequencing data containing $\mathrm{C} \rightarrow \mathrm{T}$ transitions aligned to the reference genome (sacCer3, version 64.2.1). A potential $\mathrm{m} 3 \mathrm{C}$ site was only called with a transition rate of $4 \%$ and read coverage of at least 40 .

\begin{tabular}{|c|c|c|c|c|c|c|}
\hline Chr & Position & $\mathrm{C} \rightarrow \mathrm{T}$ & Reference & Ratio & Feature & Gene \\
\hline chrV & 86519 & 104 & 5 & 0,95 & mRNA & MTC7 \\
\hline chrlll & 162694 & 94 & 16 & 0,85 & mRNA & YCR024C-B \\
\hline chrlV & 1402305 & 71 & 13 & 0,85 & mRNA & RPL27B \\
\hline chrlV & 1153577 & 82 & 36 & 0,69 & mRNA & YDR341C \\
\hline chrll & 323668 & 27 & 18 & 0,60 & mRNA & QDR3 \\
\hline chrXIII & 139652 & 23 & 19 & 0,55 & mRNA & ERV41 \\
\hline chrXI & 46776 & 2538 & 2395 & 0,51 & tRNA & $\mathrm{T}(\mathrm{CGU})$ \\
\hline chrXVI & 827581 & 66 & 76 & 0,46 & mRNA & YPR148C \\
\hline chrV & 41712 & 83 & 96 & 0,46 & mRNA & PRB1 \\
\hline chrXIII & 159468 & 82 & 149 & 0,35 & mRNA & SML1 \\
\hline chrVII & 978952 & 19 & 65 & 0,23 & mRNA & LSC2 \\
\hline chrVIII & 303572 & 27 & 94 & 0,22 & mRNA & TRA1 \\
\hline chrll & 685511 & 9 & 37 & 0,20 & mRNA & ARC40 \\
\hline chrXV & 1017134 & 17 & 73 & 0,19 & mRNA & PRT1 \\
\hline chrXV & 796725 & 8 & 44 & 0,15 & mRNA & ENV9 \\
\hline $\operatorname{chrXV}$ & 829817 & 8 & 46 & 0,15 & mRNA & VPH1 \\
\hline chrXII & 1004572 & 9 & 54 & 0,14 & mRNA & CNA1 \\
\hline chrX & 538585 & 223 & 1588 & 0,12 & tRNA & $\mathrm{R}(\mathrm{CCU})$ \\
\hline chrXIV & 349743 & 12 & 109 & 0,10 & mRNA & PGA2 \\
\hline chrV & 81963 & 5 & 52 & 0,09 & mRNA & RAD23 \\
\hline chrVI & 75912 & 6 & 74 & 0,08 & mRNA & HAC1 \\
\hline chrXIV & 654597 & 3 & 37 & 0,08 & mRNA & $\mathrm{ACC} 1$ \\
\hline chrXIV & 661501 & 22 & 273 & 0,07 & mRNA & ACC1 \\
\hline chrlX & 369738 & 4 & 52 & 0,07 & mRNA & PAN1 \\
\hline chrVIII & 292455 & 3 & 45 & 0,06 & mRNA & HXT1 \\
\hline chrVIII & 467020 & 17 & 266 & 0,06 & tRNA & $\mathrm{T}(\mathrm{UGU})$ \\
\hline chrV & 138519 & 3 & 54 & 0,05 & CUT/XUT & \\
\hline chrXV & 282163 & 4 & 76 & 0,05 & tRNA & $G(G G C)$ \\
\hline
\end{tabular}




\begin{tabular}{|l|l|l|l|l|l|l|}
\hline chrVII & 519348 & 3 & 58 & 0,05 & mRNA & MSB2 \\
\hline chrXI & 464423 & 6 & 120 & 0,05 & mRNA & PRY2 \\
\hline chrXIII & 887067 & 5 & 100 & 0,05 & mRNA & GAS1 \\
\hline chrVII & 111292 & 5 & 106 & 0,05 & mRNA & YGL204C \\
\hline chrIII & 227973 & 45 & 1015 & 0,04 & tRNA & S(CGA) \\
\hline chrVII & 866510 & 3 & 72 & 0,04 & mRNA & TYS1 \\
\hline chrX & 519511 & 3 & 72 & 0,04 & mRNA & SSC1 \\
\hline chrXII & 818154 & 13 & 351 & 0,04 & XUT & \\
\hline
\end{tabular}


7.1.3 Spike-in sequences

7.1.3.1 Spike-in 2

GGGUGCUUUAACAAGAGGAAAUUGUGUUUUUGCCAAUUUAAGACCUAA UUUAAUAGUUAAACCAUUAACCUUAGUUGUUCCAAGGCAUAAUAUAGA GAGUGAGAUACAGGAUGAGCUAUUUCAGGGAGUUAUUCAGUAUGCAG UUGCCAAGGCAGUUGCUGAUUUAGAUUUAGAUGAAGAUUUAAAGGUU GUUGUCUCUGUUAAUGUCCCAGAGGUUCCAAUAACCAAUUUAAAUAAA AGAAAACUCUUCCAAUACUUCUAUGCCUCAGCAAAGUUAGCUAUAAAC AGAGCUUUAAAUGAAUAUCCUUCAAAAGAGAAGGUAAAGAAAGAGAAAU AUAGAGCUUUGCAUCCAUUAGUUGGAUUUAGGGAUGUUAGAUUGGAG UAUCCUCCAUAUCUACAAAUUGCUUUGGAUGUCCCAACUAUGGAGAAU UUGGAAUUUUUGUUACAAACAAUUCCAAAUAGCGACCACAUCAUCUUA GAGGCUGGAACACCACUAAUUAAAAAGUUUGGUUUAGAGGUUAUUGAA AUAAUGAGAGAAUAUUUUGAUGGCUUUAUUGUUGCUGAUUUAAAAACC UUAGACACUGGAAGGGUUGAGGUAAGAUUGGCAUUUGAAGCAACAGC UAAUGCAGUGGCAAUAAGUGGAGUAGCACCAAAAUCAACAAUAAUUAA AGCUAUCCACGAAUGUCAAAAAUGUGGUUUAAUCAGCUAUUUGGAUAU GAUGAACGUCUCUGAACCUCAAAAAUUAUAUGAUUCAUUAAAAUUAAAG CCAGAUGUUGUUAUCUUGCAUAGAGGGAUUGAUGAGGAGACAUUUGG AAUUAAAAAGGAAUGGAAAUUUAAGGAAAACUGCUUAUUAGCAAUUGC UGGAGGAGUUGGUGUGGAGAAUGUUGAAGAGCUUUUAAAAGAAUAUC AAAUAUUAAUCGUUGGUAGAGCAAUUACAAAAUCAAAAGACCCAGGAA GAGUAAUUAGGAUUUUAUAAACAAGAUGG

\subsubsection{Spike-in 4}

GGGUUUCGACGUUUUGAAGGAGGGUUUUAAGUAAUGAUCGAGAUUGA AAAACCAAAAAUCGAAACGGUUGAAAUCAGCGACGAUGCCGAAUUUGG UAAGUUUGUCGUAGAGCCACUUGAGCGUGGAUAUGGUACAACUCUGG GUAACUCCUUACGUCGUAUCCUCUUAUCCUCACUCCCUGGUGCCGCU GUAACAUCAAUCCAGAUAGAUGGUGUACUGCACGAAUUCUCGACAAUU GAAGGCGUUGUGGAAGAUGUUACAACGAUUAUCUUACACAUUAAAAAG CUUGCAUUGAAAAUCUACUCUGAUGAAGAGAAGACGCUAGAAAUUGAU GUACAGGGUGAAGGAACUGUAACGGCAGCUGAUAUUACACACGAUAGU GAUGUAGAGAUCUUAAAUCCUGAUCUUCAUAUCGCGACUCUUGGUGA GAAUGCGAGUUUCCGAGUUCGCCUUACUGCUCAAAGAGGACGUGGGU AUACGCCUGCUGACGCAAACAAGAGAGGCGAUCAGCCAAUCGGCGUG AUUCCGAUCGAUUCUAUCUAUACGCCAGUUUCCCGUGUAUCUUAUCAG GUAGAGAACACUCGUGUAGGCCAAGUUGCAAACUAUGAUAAACUUACA CUUGAUGUUUGGACUGAUGGAAGCACUGGACCGAAAGAAGCAAUUGC GCUUGGUUCAAAGAUUUUAACUGAACACCUUAAUAUAUUCGCUGGUUU AACUGACGAAGCUCAACAUGCUGAAAUCAUGGUUGAAGAAGAAGAAGA UCAAAAAGAGAAAGUUCUUGAAAUGACAAUUGAAGAAUUGGAUCUUUC UGUUCGUUCUUACAACUGCUUAAAGCGUGCGGGUAUUAACACGGUUC AAGAGCUUGCGAACAAGACGGAAGAAGAUAUGAUGAAAGUUCGAAAUC UAGGACGCAAAUCACUUGAAGAAGUGAAAGCGAGACUAGAAGAACUUG 
GACUCGGACUUCGCAAAGACGAUUGACUAGUUUCCCUUGUGAACUAG GAUUU UCCCGGGUAC

\subsubsection{Spike-in 5}

GGGACUGUCCUUUCAUCCAUAAGCGGAGAAAGAGGGAAUGACAUUGU UCUUACACGGCACAAGCAGACAAAAUCAACAUGGUCAUUUAGAAAUCG GAGGUGUGGAUGCUCUCUAUUUAGCGGAGAAAUAUGGUACACCUCUU UACGUAUAUGAUGUGGCUUUAAUACGUGAGCGUGCUAAAAGCUUUAAG CAGGCGUUUAUUUCUGCAGGGCUGAAAGCACAGGUGGCAUAUGCGAG CAAAGCAUUCUCAUCAGUCGCAAUGAUUCAGCUCGCUGAGGAAGAGG GACUUUCUUUAGAUGUCGUAUCCGGAGGAGAGCUAUAUACGGCUGUU GCAGCAGGCUUUCCGGCAGAACGCAUCCACUUUCAUGGAAACAAUAAG AGCAGGGAAGAACUGCGGAUGGCGCUUGAGCACCGCAUCGGCUGCAU UGUGGUGGAUAAUUUCUAUGAAAUCGCGCUUCUUGAAGACCUAUGUAA AGAAACGGGUCACUCCAUCGAUGUUCUUCUUCGGAUCACGCCCGGAG UAGAAGCGCAUACGCAUGACUACAUUACAACGGGCCAGGAAGAUUCAA AGUUUGGUUUCGAUCUUCAUAACGGACAAACUGAACGGGCCAUUGAAC AAGUAUUACAAUCGGAACACAUUCAGCUGCUGGGUGUCCAUUGCCAUA UCGGCUCGCAAAUCUUUGAUACGGCCGGUUUUGUGUUAGCAGCGGAA AAAAUCUUCAAAAAACUAGACGAAUGGAGAGAUUCAUAUUCAUUUGUA UCCAAGGUGCUGAAUCUUGGAGGAGGUUUCGGCAUUCGUUAUACGGA AGAUGAUGAACCGCUUCAUGCCACUGAAUACGUUGAAAAAAUUAUCGA AGCUGUGAAAGAAAAUGCUUCCCGUUACGGUUUUGACAUUCCGGAAAU UUGGAUCGAACCGGGCCGUUCUCUCGUGGGAGACGCAGGCACAACUC UUUAUACGGUUGGCUCUCAAAAAGAAGUGGAUAAGCUGUACAAUCGUU UCAUC AUUCGGCGUGCG

\subsubsection{Spike-in 8}

GGGGAUGUCCUUGGACGGGGUGGCGCAGUAUUACUGCAAGAGAGCG GACAGAUUAGUGUGUUGGAGCCGACACAUCAAAGGUUCGUCCGGGGA CCGAUCUGCAGCCUACGGGACAUUUAUCCGUAAAAGCAUGGCGCUGU UUCGUACUUAUCGGAGGCCAGGUAUCGUCGCGGCGAGUCUCCCCGAC GACGGAGAUGGGCGUUACUAUCUGGGCCGUCUCGUACUCUGUUACUU GGCACAGAUGCGAGCCCUCGUAAUGUGCAUCAGCUAAGGGCGAUAUU AUAAUGCGACGUUUGUACGGAUUCGUUACUAACGUGUUGGACGCUAG UGGAAUAUGUGUCGUUGGUUAGCCUACCCAUGGCUUUCGCGGCGACA CAUGCUUAGACUCUUUCAAAACUUCGGUGAAGUUCACUCAAGCCGCGG AGCGCCGUCGUAAUUCACUAGGGAUGGCGGUACCCGUGCCCGUCCGA UUCGUAGCAACCUGCAUCACGAUUUUGUCUUCGGGCGACUUAUCAGA UACGGUAAUGUAAAUACCUGGCAUUUGGGCACUUCUUGCGUUUAAGC GGGAAAGAUCGCGAGGGCCCGCUAUUUGCGAUACUUCCCAUGUCGGU GCCGUCGCCUCUAUGUACUCGGAGACGUUAAUGCAGAGGCUAAGGAC AAUUUACCAUGACUCGGUAAUCCGUUCGUCAAGCAGGUAGCUCGAGU CUCCCCACGGACACGUAGUGGGUUUGUAACGAUCGAUACCGAGUCUU 
UUUGUCUAGUAGAACCAACCAACCAUUAAGGAGUUCACUAGCACAUCU UUGCGACCCGAUCGUCCGUGUGUCGCGUAAUACUUUUGUUAUGACGA GACAUACGCUCAAGCCCUGGGUAGCUAGUCGCGGAGGCACGUUACCG CGCACAACCCCUAUUCGUUUACAUGUACAUCGCAUCUGAGGUAGUACA CUUCCGGCGUACGUGAGUAUUUGCGCGUAAUAAGCGCGUGUUUAGCU GAUCCCCUCUCGUAUCGAGGUUAAGGCAGAUUAGUGCCCAGUAAUUG CGUUUUUUUGUCGUUGUCGCAGAACGCGAUUUGCUCCGAAAGC

\subsubsection{Spike-in 9}

GGGCCAGAUUACUUCCAUUUCCGCCCAAGCUGCUCACAGUAUACGGG CGUCGGCAUCCAGACCGUCGGCUGAUCGUGGUUUUACUAGGCUAGAC UAGCGUACGAGCACUAUGGUCAGUAAUUCCUGGAGGAAUAGGUACCAA GAAAAAAACGAACCUUUGGGUUCCAGAGCUGUACGGUCGCACUGAACU CGGAUAGGUCUCAGAAAAACGAAAUAUAGGCUUACGGUAGGUCCGAAU GGCACAAAGCUUGUUCCGUUAGCUGGCAUAAGAUUCCAUGCCUAGAU GUGAUACACGUUUCUGGAAACUGCCUCGUCAUGCGACUGUUCCCCGG GGUCAGGGCCGCUGGUAUUUGCUGUAAAGAGGGGCGUUGAGUCCGU CCGACUUCACUGCCCCCUUUCAGCCUUUUGGGUCCUGUAUCCCAAUU CUCAGAGGUCCCGCCGUACGCUGAGGACCACCUGAAACGGGCAUCGU CGCUCUUCGUUGUUCGUCGACUUCUAGUGUGGAGACGAAUUGCCAGA AUUAUUAACUGCGCAGUUAGGGCAGCGUCUGAGGAAGUUUGCUGCGG UUUCGCCUUGACCGCGGGAAGGAGACAUAACGAUAGCGACUCUGUCU CAGGGGAUCUGCAUAUGUUUGCAGCAUACUUUAGGUGGGCCUUGGCU UCCUUCCGCAGUCAAAACCGCGCAAUUAUCCCCGUCCUGAUUUACUGG ACUCGCAACGUGGGUCCAUCAGUUGUCCGUAUACCAAGACGUCUAAG GGCGGUGUACACCCUUUUGAGCAAUGAUUGCACAACCUGCGAUCACC UUAUACAGAAUUAUCAAUCAAGCUCCCCGAGGAGCGGACUUGUAAGGA CCGCCGCUUUCGCUCGGGUCUGCGGGUUAUAGCUUUUCAGUCUCGAC GGGCUAGCACACAUCUGGUUGACUAGGCGCAUAGUCGCCAUUCACAG AUUUGCUCGGCAAUCAGUACUGGUAGGCGUUAGACCCCGUGACUCGU GGCUGAACGGCCGUACAA CUCGACAGCCGGUGCUUGCGUUUUACCC

\subsubsection{Spike-in 12}

GGGGCACAAGUUGCUGAAGUUGCGAGAGGGGCGAUAAGUGAGGCAGA CAGGCAUAAUAUAAGAGGGGAGAGAAUUAGCGUAGAUACUCUUCCAAU AGUUGGUGAAGAAAAUUUAUAUGAGGCUGUUAAAGCUGUAGCAACUCU UCCACGAGUAGGAAUUUUAGUUUUAGCUGGCUCUUUAAUGGGAGGGA AGAUAACUGAAGCAGUUAAAGAAUUAAAGGAAAAGACUGGCAUUCCCG UGAUAAGCUUAAAGAUGUUUGGCUCUGUUCCUAAGGUUGCUGAUUUG GUUGUUGGAGACCCAUUGCAGGCAGGGGUUUUAGCUGUUAUGGCUAU UGCUGAAACAGCAAAAUUUGAUAUAAAUAAGGUUAAAGGUAGGGUGCU AUAAAGAUAAUUUAAUAAUUUUUGAUGAAACCGAAGCGUUAGCUUUGG GUUAUGAAACUCCAUGAUUUUCAUUUAAUUUUUUCCUAUUAAUUUUCU CCUAAAAAGUUUCUUUAACAUAAAUAAGGUUAAAGGGAGAGCUCUAUG 
AUUGUCUUCAAAAAUACAAAGAUUAUUGAUGUAUAUACUGGAGAGGUU GUUAAAGGAAAUGUUGCAGUUGAGAGGGAUAAAAUAUCCUUUGUGGAU UUAAAUGAUGAAAUUGAUAAGAUAAUUGAAAAAAUAAAGGAGGAUGUUA AAGUUAUUGACUUAAAAGGAAAAUAUUUAUCUCCAACAUUUAUAGAUG GGCAUAUACAUAUAGAAUCUUCCCAUCUCAUCCCAUCAGAGUUUGAGA AAUUUGUAUUAAAAAGCGGAGUUAGCAAAGUAGUUAUAGACCCGCAUG AAAUAGCAAAUAUUGCUGGAAAAGAAGGAAUUUUGUUUAUGUUGAAUG AUGCCAAAAUUUUAGAUGUCUAUGUUAUGCUUCCUUCCUGUGUUCCAG CUACAAACUUAGAAACAAGUGGAGCUGAGAUUACAGCAGA 
7.2 Abbreviations

\begin{tabular}{|c|c|}
\hline 3' UTR & $3^{\prime}$ untranslated region \\
\hline $4 \mathrm{tU}$ & 4-thiouracil \\
\hline $4 \mathrm{sU}$ & 4-thiouridine \\
\hline $5^{\prime}$ UTR & $5^{\prime}$ untranslated region \\
\hline $4 \mathrm{tU}$ & 4-thiouracil \\
\hline$A$ & Adenine \\
\hline$A^{\prime}$ & acceptor \\
\hline Abp140 & actin binding protein 140 \\
\hline $\operatorname{ac} 4 \mathrm{C}$ & $N^{4}$-acetylcytidine \\
\hline ACL & anticodon loop \\
\hline Air & $\begin{array}{l}\text { Arginine methyltransferase-interacting RING finger } \\
\text { protein }\end{array}$ \\
\hline ALKBH & alkylation repair homolog \\
\hline Arg & Arginine \\
\hline Asn & Asparagine \\
\hline ATP & Adenosine triphosphate \\
\hline $\mathrm{bp}$ & base pair \\
\hline BSA & bovine serum albumin \\
\hline Bur & bypass UAS requirement \\
\hline C & Cytosine \\
\hline $\mathrm{C} 32$ & C at position 32 \\
\hline Caf40 & Ccr4 associated factor \\
\hline $\mathrm{Cbc2}$ & cap binding complex 2 \\
\hline Ccr4 & carbon catabolite repressor 4 \\
\hline Cdc & cell division cycle \\
\hline cDNA & complementary DNA \\
\hline CDS & coding sequence \\
\hline $\mathrm{Cft} 2$ & cleavage factor 2 \\
\hline $\operatorname{chr}$ & chromosome \\
\hline Csl4 & Cep1 synthetic lethal \\
\hline \multirow[t]{2}{*}{ CMC } & $N^{3}-[N$-cyclohexyl- $N$ '- $\beta$-(4-methylmorpholinium) \\
\hline & ethylcarbodiimide \\
\hline CMV & cytomegalovirus \\
\hline CRAC & Crosslinking and cDNA analysis \\
\hline
\end{tabular}


CTD

Ctk

Ctr9

CUT

Dcp

Dhh1

Dis3

DMEM

DMSO

DNA

Dot1

DTT

E

E. coli

eCLIP

ECL

EDTA

Edc

EGTA

ERCC

f5C

FAM

FBS

FTP

FLP

G

Gbp2

GTF

$\mathrm{h}$

HEK

HEPES

hm5C

Hrb1

HRP

Hrp1

I

i6A37
C-terminal domain

Carboxy-terminal domain kinase

Cln three requiring

Cryptic unstable transcripts

mRNA decapping

DEAD box helicase homolog

chromosome disjunction

Dulbecco's Modified Eagle's Medium

Dimethylsulfoxide

Deoxyribonucleic acid

disruptor of telomeric silencing

dithiothreitol

exit

Escherichia coli

enhanced crosslinking and immunoprecipitation

enhances chemiluminescence

ethylenediaminetetraacetic acid

enhancer of mRNA decapping

ethyleneglycoltetraacetic acid

external RNA control consortium

5 -formylcytidine

fluorescein amidite

Fetal bovine serum

fat mass and obesity associated protein

flippase

Guanine

G-strand binding protein

General transcription factor

hour

human embryonic kidney

4-(2-hydroxyethyl)-1-piperazineethanesulfonic acid

5-hydroxymethylcytidine

Hypothetical RNA binding protein

Horseradish peroxidase

heterogenous nuclear ribonucleoprotein

Inosine

$N^{6}$-isopentnyladenosine at position 37 


\begin{tabular}{|c|c|}
\hline $\lg G$ & immunoglobulin G \\
\hline IPTG & Isopropyl- $\beta$ - D-thiogalactopyranosid \\
\hline Ile & Isoleucine \\
\hline Ist3 & increased sodium tolerance 3 \\
\hline $\mathrm{hm} 5 \mathrm{C}$ & 5-hydroxymethylcytidine \\
\hline $\mathrm{k}$ & kilo \\
\hline $\mathrm{kDa}$ & kilo Dalton \\
\hline KO & knockout \\
\hline L & liter \\
\hline LB & Lysogeny Broth \\
\hline LC-MS/MS & $\begin{array}{l}\text { High-performance liquid chromatography-coupled triple } \\
\text { quadrupole mass spectrometry }\end{array}$ \\
\hline LDS & sample loading buffer \\
\hline Leo1 & left open reading frame \\
\hline LIC & ligase independent cloning \\
\hline lincRNA & long intergenic non-coding RNA \\
\hline Luc7 & Lethal unless cap-binding complex is produced \\
\hline Lys & Lysine \\
\hline $\mathrm{m}$ & milli \\
\hline M & moles per liter \\
\hline$\mu$ & micro \\
\hline $\mathrm{m} 1 \mathrm{~A}$ & $N^{1}$-methyladenosine \\
\hline $\mathrm{m}^{2}{ }_{2} \mathrm{G}$ & $N^{2}-N^{2}$-dimethylguanosine \\
\hline $\mathrm{m} 3 \mathrm{C}$ & $N^{3}$-methylcytidine \\
\hline $\mathrm{m} 5 \mathrm{C}$ & 5-methylcytidine \\
\hline $\mathrm{m} 6 \mathrm{~A}$ & $N^{6}$-methyladenosine \\
\hline MBP & maltose binding protein \\
\hline Met & Methionine \\
\hline METTL & Methyltransferase-like \\
\hline Mex67 & mRNA export factor of $67 \mathrm{kDa}$ \\
\hline $\min$ & minute \\
\hline mRNA & messenger RNA \\
\hline miRNA & micro RNA \\
\hline MOPS & 3-(N-morpholino)propanesulfonic acid \\
\hline Mpe1 & mutant Pcf11 extragenic suppressor \\
\hline Mtr4 & mRNA transport \\
\hline Mud & Mutant U1 die \\
\hline
\end{tabular}


Msl5

$\mathrm{Nab}$

Nam8

NAT10

ncRNA

NDR

NGS

NMD

Not1

Nrd1

Npl3

NUT

nt

OD

ORF

$\mathrm{P}$

pA

Pab1

Paf1

PAGE

Pan2/3

PAP

PAR-CLIP

PBS

Pcf11

PCR

PEG

PIC

PMSF

PNK

$\mathrm{POI}$

Pol

Pop2

Pub1

Pus

PVDF
Mud synthetic lethal

Nuclear polyadenylated RNA binding

Nuclear accommodation of mitochondria 8

$\mathrm{N}$-acetyltransferase 10

non-coding RNA

nucleosome depleted region

Next-generation sequencing

Nonsense mediated decay

negative on TATA

Nuclear pre-mRNA Down-regulation

nuclear protein localization

Nrd1-unterminated transcript

nucleotide

optical density

open reading frame

peptidyl

poly adenylation

pA binding protein 1

RNA Pol II associated factor

Polyacrylamide gel electrophoresis

pA nuclease $2 / 3$

pA polymerase

Photoactivatable ribonucleoside enhanced crosslinking and immunoprecipitation

Phosphate buffered saline

protein 1 of cleavage and polyadenylation factor 1

polymerase chain reaction

Polyethylene glycol

Pre-initiation complex

Phenylmethylsulfonylfluoride

polynucleotide kinase buffer

Protein of interest

RNA-dependent Polymerase

PGK promoter directed overproduction

poly uridine binding

Pseudouridine synthase

Polyvinylidene difluoride 


\begin{tabular}{|c|c|}
\hline Rai1 & Rat1 interacting protein \\
\hline Rat1 & ribonucleic acid trafficking \\
\hline RNA & ribonucleic acid \\
\hline Rna15 & pA mRNA metabolism \\
\hline rRNA & ribosomal RNA \\
\hline Rpb1 & RNA polymerase \\
\hline rpm & rounds per minute \\
\hline $\mathrm{RRACH}$ & $R$ is $A$ or $G$; $H$ is $A, C$ or $U$ \\
\hline Rrp & Ribosomal RNA Processing \\
\hline RT & reverse transcription \\
\hline Rtf1 & restores TBP function \\
\hline Rtt103 & Regulator of Ty1 transposition \\
\hline Sen1 & Splicing endonuclease \\
\hline S & Sedimentation coefficient \\
\hline S2P & Serine-2 phosphorylation \\
\hline S5P & Serine-5 Phosphorylation \\
\hline S. cerevisiae & Saccharomyces cerevisiae \\
\hline SAM & S-adenosylmethionine \\
\hline SCM & Synthetic complete medium \\
\hline SDS & Sodium dodecyl sulfate \\
\hline Set & SET domain containing \\
\hline SGD & Saccharomyces genome database \\
\hline Smg6 & suppressor of morphological defects \\
\hline Ski & super killer \\
\hline SNP & single nucleotide polymorphism \\
\hline Snp1 & U1 small nuclear ribonucleoprotein \\
\hline snRNA & small nuclear RNA \\
\hline snoRNA & small nucleolar RNA \\
\hline Spt & suppressor of Ty's \\
\hline Sub2 & suppressor of Brr1-1 \\
\hline SUT & stable unannotated transcript \\
\hline t6A37 & $N^{6}$-threonyladenosine at position 37 \\
\hline $\mathrm{T}$ & Thymine \\
\hline TAP & Tandem affinity purification \\
\hline TBP & TATA box binding protein \\
\hline TE & Translational efficiency \\
\hline TEV & Tobacco etch virus \\
\hline
\end{tabular}


Tho2

Thr

TRAMP

Trf

Tris

tRNA

Trm140

TRUB1

tSNE

TSS

TTS

TT-seq

$\Psi$

U

UMI

Upf

UV

$\mathrm{V}$

v/v

$w / v$

WT

WTAP

YSPSTPS

YPD

Yra1

YTH

Yth1

YTHDC

YTHDF

Xrn1

XUT suppressor of the transcriptional defect of $\mathrm{Hpr} 1$ by

overexpression

Threonine

Trf4/5-Air1/2-Mtr4 polyadenylation complex

topoisomerase one-related function

tris(hydroxymethyl)aminomethane

transfer RNA

tRNA-methyltransferase 140

tRNA pseudouridine synthase $B$

t-Distributed Stochastic Neighbor Embedding

transcription start site

transcription termination site

Transient transcriptome sequencing

Pseudouridine

Unit

unique molecular identifier

UP frameshift

Ultra violet

Volt

volume per volume

weight per volume

wildtype

Wilms tumor suppressor gene

Tyrosine-Serine-Proline-Serine-Threonine-Proline-

Serine

Yeast extract peptone dextrose

Yeast RNA annealing protein

YT521-B homology

Yeast thirty kDa homolog

YTH domain containing

YTH domain family

exoribonuclease 1

Xrn1-unterminated transcripts 


\subsection{List of Figures}

Figure 1: Translation by the ribosome. $-6-$

Figure 2: Schematic overview of RNA degradation ............................... 10 -

Figure 3: Model of the exosome complex........................................... 12 -

Figure 4: Modified nucleosides in the clover-leaf structure of the eukaryotic tRNA. $-16-$

Figure 5: Schematic representation of known chemical modifications mapped in eukaryotic mRNA transcripts. ........- 17 -

Figure 6: Structure of the $\mathrm{N}^{3}$-methylcytosine $(\mathrm{m} 3 \mathrm{C})$ modification............. 23 -

Figure 7: Number of PAR-CLIP cross-link sites and replicate correlation.

Figure 8: Distribution of degradation factor cross-link sites over the yeast transcriptome.

Figure 9: Metagene analysis of degradation factor binding on protein-coding mRNAs. $-63-$

Figure 10: Surveillance of aberrant nuclear non-coding RNA by components of the exosome and the TRAMP4 complex

Figure 11: Global co-occupancy and co-localization analysis reveals unexpected cooperation between factors from different complexes and pathways.

Figure 12: Binding preferences reveal a link between decappingmediated degradation and translation.

Figure 13: Location and recruitment of the decapping complex Dcp1/Dcp2 and decapping enhancers Edc3, Dhh1, and Edc2.

Figure 14: Schematic overview of PAR-CLIP and m3C-CLIP protocol. $-85-$

Figure 15: PAR-CLIP of Trm140 analyzed by Western Blot and phosphor imaging

Figure 16: Identification of mutational signature of $\mathrm{N}^{3}$-methylcytidine (m3C) modification.

Figure 17: $\mathrm{m} 3 \mathrm{C}$ modification causes reverse transcription block shown by primer extension assay on tRNA Serine.

Figure 18: tRNA Arginine (CCU) contains $\mathrm{m} 3 \mathrm{C}$ at $\mathrm{C} 32$ in S. cerevisiae. $-92-$

Figure 19: Presence of $\mathrm{N}^{3}$-methylcytidine modification in mRNA............. 94 -

Figure 20: $\mathrm{m} 3 \mathrm{C}$ modification loss changes ribosome dynamics................ - 96 -

Figure 21: Trm140 co-localizes with various RNA degradation factors.

Figure 22: Spearman correlation of 4tU-seq data for Trm140 KO and WT cells.

Figure 23: Functional characterization of $\mathrm{m} 3 \mathrm{C}$ modification loss by using metabolic RNA labeling and sequencing in S. cerevisiae.

Figure 24: PAR-CLIP of METTL8-3xFLAG in FIp-In ${ }^{\text {TM }}$ T-REX ${ }^{\text {TM }} 293$ cells. 
Figure 25: METTL8 PAR-CLIP reveals localization of $\mathrm{m} 3 \mathrm{C}$ modification in mRNA.

Figure 26: Schematic drawing of Watson Crick base pairing between unmodified $\mathrm{C}$ and $\mathrm{G}(\mathrm{A})$ as well as $\mathrm{H}-\mathrm{H}$ repulsion of $m 3 C$ and $G(B)$.

Figure 27: Illustration of the m6A switch model................................ - 110 -

Figure 28: Different transcript classes have comparable U-content....... - 141 -

Figure 29: Metagene profiles of yeast RNA degradation factors centered on translation start and stop sites in comparison to TIF-annotated TSS and pA sites.

Figure 30: Distributions of transcript length, half-life, expression level and transcript optimality for yeast mRNAs.

Figure 31: Occupancies of deadenylation factors (Ccr4, Pop2, Not1, Caf40, Pan2, and Pan3) compared to transcript length, optimality, expression level, and half-life.

Figure 32: Occupancies of decapping factors (Dcp2, Dcp1, Edc2, Edc3, and Dhh1) compared to transcript length, optimality, expression level, and half-life.

Figure 33: Occupancy of Xrn1 compared to transcript length, optimality, expression level, and half-life.

Figure 34: Occupancies of exosome components (Rrp6, CsI4, Rrp40, Rrp4, and Rrp44) compared to transcript length, optimality, expression level, and half-life.

Figure 35: Occupancies for components of the TRAMP complex (Air1, Trf5, Mtr4, Air2, and Trf4) compared to transcript length, optimality, expression level, and half-life.

Figure 36: Occupancies for components of the Ski complex (Ski2, Ski3, Ski7, and Ski8) compared to transcript length, optimality, expression level, and half-life.

Figure 37: Occupancies for components of the NMD pathway (Upf1, Upf2, Upf3, and Nmd4) compared to transcript length, optimality, expression level, and half-life.

Figure 38: Correlation between binding to degradation factors and transcript length, codon-optimality, expression, and halflife.

Figure 39: Metagene profiles for subunits of the TRAMP complex on snoRNA genes. $-149-$

Figure 40: Comparison of binding profiles on genes containing upstream sense NUTs with all mRNAs. $150-$

Figure 41: Co-localization coefficients for 75 RNA binding factors. $-151-$ 


\subsection{List of Tables}

Table 1: Bacterial strains used in this study..................................... 27 -

Table 2: Yeast strains used in this study....................................... 27 -

Table 3: Human cell lines used in this study. .................................... 28 -

Table 4: Growth media used in this study. ......................................... 29 -

Table 5: Media supplements used in this study................................. $29-$

Table 6: Spike-ins used in this study provided by Dr. Kristina Zumer........ - 30 -

Table 7: Primers and oligonucleotides used in this study were purchased from IDT......................................................... 30 -

Table 8: List of thermal cycler programs used in this study................... 31 -

Table 9: List of plasmids used in this study.................................... 32 -

Table 10: List of buffers and solutions used in this study. ..................... 32 -

Table 11: List of antibodies used in this study..................................... $33-$

Table 12: Biological replicates for yeast PAR-CLIP experiments............. 37 -

Table 13: Overview of RNA processing factors and their respective published PAR-CLIP experiments. ................................. - 152 -

Table 14: High confidence m3C modification sites........................... 153 - 
Appendix

- 168 - 